\title{
Nanometric Surface Probing Through Ultra-Cold Atoms
}

Zur Erlangung des akademischen Grades eines

\author{
DOKTOR-INGENIEURS
}

an der Fakultät für

Elektrotechnik und Informationstechnik

des Karlsruher Instituts für Technologie (KIT)

genehmigte

DISSERTATION

\author{
von \\ Ali Khan, Murtaza \\ geb. in Rawalpindi, Pakistan
}

Tag der mündlichen Prüfung: 13.07.2017

Hauptreferent: Prof. Dr. Uli Lemmer

Korreferent: Prof. Dr. Francesco S. Cataliotti (Università degli Studi di Firenze, Italy) 


\begin{abstract}
In this thesis we simulate and design an experimental set up that is going to be used in many applications of applied physics and will give us many answer to unsolved puzzles. This experimental set up is going to feature for the first time the ability to interchange an atom chip frequently and rapidly. The setup also brings two emerging fields, ultracold atoms and nanophotonics, together on an optical table.
\end{abstract}

First, we calculated and simulated the cold atom set-up. Then we simulated and designed an atom chip capable of bringing ultra-cold atoms to sub-micron distances to the chip surface. This new apparatus will present very first ideas on how to use the unique combination of cold atom technology with interchangeable photonics components, both, for basic research and for technological applications to modern information technologies. Furthermore, we can readily exchange the chip to test new components without the need for complicated high-vacuum procedures.

The setup will be paired with photonic structures built on-chip for the detailed study of matter-light interactions and, e.g., a depth investigation of dispersion forces (the Casimir interaction and the van der Waals interaction) between cold atoms and carbon nanotubes, that can be measured by scanning probe microscopy. This will allow the integration of photonic waveguides atom chip and will open a new door for modern information technologies.

Thesis effort is a joint venture project between the European Laboratory for Non-Linear Spectroscopy (LENS), Florence and the Karlsruhe Institute of Technology (KIT), Karlsruhe, and thrives to bring together experiences in cold atom physics and photonics. 


\section{Zusammenfassung}

In dieser Arbeit haben wir einen experimentellen Aufbau simuliert und entworfen, der in vielen Anwendungen der angewandten Physik eingesetzt wird und uns viele Antworten zu ungelösten Fragestellungen gibt. Diese experimentelle Einrichtung ermögliches zum ersten Mal, den eingesetzten Atom-Chip häufig und schnell auszutauschen. Das Setup bringt auf einem einzigen optischen Tisch zwei aufstrebende Felder (Atom-Licht Interaktion) zusammen.

Zuerst wurden die notwendigen Berechnungen und Simulationen zur Erzeugung und Speicherung der kalten Atome durchgeführt. Dazu haben wir den Atom-Chip entworfen und simuliert. Auf diesem Chip werden die kalten Atome bis auf Mikrometer an seine Oberfläche herangeführt. Dieses neue Gerät präsentiert erste Ideen, wie die einzigartige Kombination von Kalter-Atom-Technologie und austauschbaren Photonik-Komponenten eingesetzt werden kann, um sowohl der Grundlagenforschung als auch der Anwendung in moderner Informationstechnologie zu dienen. Das besondere Merkmal des hier vorgestellten Aufbaus ist, dass der Atom-Chip entsprechend den aktuellen Anforderungen schnell ausgetauscht werden kann, ohne dass das Hochvakuum-System komplett belüftet werden muss.

Der Aufbau des Atom-Chips wird neben den magnetischen Elementen relevant zur Erzeugung kalter Atomgase mit integrierten photonischen Strukturen versehen sein. Diese Kombination ermöglicht eine detaillierte Untersuchung der Materie-Licht-Wechselwirkung. Weiterhin können, z.B., Dispersionskräfte (die Casimir- und van-der-Waals-Wechselwirkung) zwischen den kalten Atomen und Kohlenstoff-Nanoröhrchen betrachtet werden. Sie können durch Rastersondenmikroskopie gemessen werden und könnten neue Wege der modernen Informationstechnologie aufzeigen.

Die vorliegende Doktorarbeit ist ein Joint-Venture-Projekt zwischen dem Europäischen Laboratorium für Nichtlineare Spektroskopie (LENS), Florenz und dem Karlsruher Institut für Technologie (KIT), Karlsruhe, dient der Zusammenführung von Erfahrungen der Atomphysik und der Photonik. 
Dedicated to my

Late Father: Azmat Ali Khan 


\section{Acknowledgements}

On Monday 7th January 2013 between 10 A.M. to 11 A.M. I entered LENS institute. On reception I asked for the Prof. Francesco Saverio Cataliotti's room. His room is situated on first floor in LENS. I knocked his door and as usual he was busy understanding the bizarre world of quantum optics. That was my first encounter with Francesco. I was very scared that he may ask me some difficult questions regarding atom physics and atom chip that I hardly met in my life before. But fortunately it was other way around, he started to talk to me like we been colleague since long time. That was my beginning in $\mathrm{PhD}$ in atomic physics. First of all I want to thank to Francesco who pushed and helped me to work in this world of atomic physics. I hardy knew about atomic physics and atom chip in beginning but with the help of Francesco I somehow understand the meaning of atomic physics. I really want to thank Prof. Francesco Saverio Cataliotti for accepting me first as a PhD student and then help me during every single step of my PhD. Without him it would be very difficult for me to complete this milestone.

I would like to thank to Prof. Dr. Wolfram Pernice during my second phase of PhD in KIT University. He helped me a lot during my stay in KIT and I learned many new ideas from him regarding Atom Chip simulation, fabrication and design.

I am also hugely appreciative of Dr. Florian Schafer, especially for sharing his taxonomic expertise so willingly, and for being so dedicated all the time. He showed me a correct path and guided me throughout my research. Without his effort it would have been difficult for me to achieve this milestone.

Also heartful thanks to my amazing Aunt Asma and my colleague Rameez for proofreading this thesis. Also thanks full to Frau Fouladi for correcting the German abstract of thesis.

A special thanks to my family. Words cannot express how grateful I am to my late father. Special thanks to my mother for all of the sacrifices that you've made on my behalf. Your prayer for me was what sustained me thus far. My special brother Saulat and his wife and more specially my sisters Sadaf and Fayza.

I would also like to thank to my Uncle Yousuf for financially supporting me and always encouraging me during my studies. A special thanks to all my great cousins.

I would also like to thank all of my friends and family members who supported me in writing, and intend me to strive towards my goal. 
October 7, 2017 


\section{Contents}

1. Introduction 9

1.1. Background . . . . . . . . . . . . . . . . . . . 9

1.2. About this thesis . . . . . . . . . . . . . . . . . . . . . 10

\begin{tabular}{ll}
\hline 2. Theory of Laser Cooling & 13
\end{tabular}

2.1. Introduction . . . . . . . . . . . . . . . . . . . . . . . 13

2.2. Properties of Rubidium $(\mathrm{Rb})$. . . . . . . . . . . . . . . . . 14

2.2.1. Rubidium (Rb) Hyperfine Structure . . . . . . . . . . . . . . . 15

2.2.2. Fine structure . . . . . . . . . . . . . . . . . . . . . 15

2.2.3. Hyperfine Levels . . . . . . . . . . . . . . . . . . . 16

2.3. Atom-Light Interaction $\ldots \ldots \ldots \ldots$

2.3.1. Optical dipole force (Conservative) . . . . . . . . . . . . . . . . . 20

2.3.2. Spontaneous Force (Dissipative) . . . . . . . . . . . . . . . . 20

2.4. Laser Cooling . . . . . . . . . . . . . . . . . . . . . . . . . . 21

2.5. Magneto Optical trapping (MOT) . . . . . . . . . . . . . . . 24

2.6. Optical Molasses . . . . . . . . . . . . . . . . . . . . . 27

2.7. Mechanical effects that make MOT really work . . . . . . . . . . . . 28

2.7.1. Doppler Cooling . . . . . . . . . . . . . . . . . . . . . 28

2.7.2. Forming a trap . . . . . . . . . . . . . . . . . 31

2.8. MOT set-up . . . . . . . . . . . . . . . . . . . . . . . . 34

2.9. Difference between traditional and Mirror MOT . . . . . . . . . . . . . . . 38

2.9.1. Working of Mirror-MOT . . . . . . . . . . . . . . . . . . . . 39

3. Experimental Set-up for Cold Atoms 43

3.1. Introduction . . . . . . . . . . . . . . . . . . . . . . 43

$3.2 . \quad$ Vacuum System $\ldots \ldots \ldots$. . . . . . . . . . . . . . . . . . 43

3.3. UHV Glass cell with different vacuum components . . . . . . . . . . . . . . 45 
3.4. Optics for the Experimental Set-up . . . . . . . . . . . . . . . . 47

3.5. BIAS and Anti-Helmholtz Coils . . . . . . . . . . . . . . . . 52

\begin{tabular}{ll}
\hline 4. Atom Chip & 62
\end{tabular}

4.1. Introduction . . . . . . . . . . . . . . . . . . . . . . 62

4.2. Silicon Photonics . . . . . . . . . . . . . . . . . . . . . . 63

4.3. Nano-wire Waveguides on SOI . . . . . . . . . . . . . . . . . . . . 63

4.3.1. Propagation in Nano-waveguide . . . . . . . . . . . . . . . . . . 64

4.3.2. TE and TM polarization in Nano-waveguide . . . . . . . . . . . . . 66

4.3.3. Loss in Optical Nano-waveguide . . . . . . . . . . . . . . . . . 71

4.3.4. Nano-waveguide Structure . . . . . . . . . . . . . . . . . . . . 74

4.3.5. Coupling Light from fiber to Nano-waveguide . . . . . . . . . . . . 78

4.3.6. Tapered Spot-size Convertors . . . . . . . . . . . . . . . . . . . . . 80

4.3.7. Grating-assisted Directional Coupler . . . . . . . . . . . . . . . . . 80

4.3.8. Mechanism of Grating Couplers . . . . . . . . . . . . . . . . . . 81

4.4. Fabrication of Silicon Waveguide Devices . . . . . . . . . . . . . . . . . . 83

4.4.1. Nano-waveguide Fabrication . . . . . . . . . . . . . . . . . . . . . 84

4.4.2. Grating Coupler Fabrication . . . . . . . . . . . . . . . . . . . . 84

4.4.3. Optical Lithography . . . . . . . . . . . . . . . . . . . . . 84

4.4.4. Electron Beam Lithography . . . . . . . . . . . . . . . . . . . . 87

4.4.5. Interference Lithography . . . . . . . . . . . . . . . . . . . . . 88

4.4.6. Dry Etching . . . . . . . . . . . . . . . . . . . . . . 88

5. Atom Chip: Design, Simulation and Fabrication 89

5.1. Introduction . . . . . . . . . . . . . . . . . . . . . . . 89

5.2. Design of the Atom Chip . . . . . . . . . . . . . . . . . . . . 89

5.2.1. Parameters of the Atom Chip . . . . . . . . . . . . . . . . . . . . 90

5.3. Simulation of Atom Chip . . . . . . . . . . . . . . . . . . . . 91

5.4. Fabrication of Atom Chip . . . . . . . . . . . . . . . . . . 96 
6. Conclusion and Future Work 102

6.1. Experiment status . . . . . . . . . . . . . . . . 102

$6.2 . \quad$ Application . . . . . . . . . . . . . . . . . . . . 102

6.3. Conclusion . . . . . . . . . . . . . . . . . . 103

6.4. Future Work . . . . . . . . . . . . . . . . . . . . 103

\begin{tabular}{ll}
\hline References & 115
\end{tabular}

\begin{tabular}{ll}
\hline A. Appendix: Vacuum Design & 129
\end{tabular}

B. Appendix: Experiment Design 133 


\section{List of Figures}

1. $\quad$ Figure showing the Rubidium . . . . . . . . . . . . . . . . . . 17

2. $\quad$ Rubidium $\left({ }^{85} \mathrm{Rb}\right)$ hyperfine splitting levels $\ldots \ldots \ldots$. . . . . . . . . . 19

3. Atom-photon interaction . . . . . . . . . . . . . . . . . . . . . 21

4. Hydrogen energy level detailed structure . . . . . . . . . . . . . . . . . . 22

$5 . \quad$ Hyper fine splitting for an atom . . . . . . . . . . . . . . . 23

6. $\quad$ Energy level diagram of two level atom . . . . . . . . . . . . . . . . . . . 24

7. 1-D MOT having a transition . . . . . . . . . . . . . . 27

8. $\quad$ Doppler cooling graph $\ldots \ldots \ldots$. . . . . . . . . . . . . . . 30

9. Rubidium ${ }^{85} \mathrm{Rb}$ level structure . . . . . . . . . . . . . . . . . . . . . . 31

10. Magnetic field generated by anti-Helmholtz coils . . . . . . . . . . . . . . 33

11. DFB laser scheme . . . . . . . . . . . . . . . . . . . . . . . . . . 34

12. Schematic of Rb atom set-up . . . . . . . . . . . . . . . . 35

13. $\quad$ Master Laser (DFB) generated the frequency . . . . . . . . . . . . . . . . 36

14. Traditional and mirror MOT . . . . . . . . . . . . . . . . . . . . . . 40

15. Set-up for Pyramidal MOT . . . . . . . . . . . . . . . . . . 41

16. Schematic representation of mirror-MOT . . . . . . . . . . . . . . . 41

17. UHV Glass cell . . . . . . . . . . . . . . . . . . . . . . . . . . . . 44

18. Cell with four cross pipe . . . . . . . . . . . . . . . . . . 45

19. Cell attached with other equipments . . . . . . . . . . . . . . . . 46

20. Cell with metal rod . . . . . . . . . . . . . . . . . . . . . . 47

21. Linear shift mechanism . . . . . . . . . . . . . . . . . . . . . . . . . . . . . 48

22. Hyperfine structure of ${ }^{87} \mathrm{Rb}$ D2 line . . . . . . . . . . . . . . . . . . . . . . 49

23. Photo of the laser-system box . . . . . . . . . . . . . . . 50

24. Main system as used by the microBEC experiment . . . . . . . . . . . 51

25. Model of the one rectangular coil . . . . . . . . . . . . . . . . . 53

26. Model of the two rectangular coils . . . . . . . . . . . . . . . 54 
27. Anti-Helmholtz coils . . . . . . . . . . . . . . . . . 57

28. Plot for Anti-Helmholtz coils . . . . . . . . . . . . . . . . . . 58

29. BIAS coils . . . . . . . . . . . . . . . . . . . 60

30. Main set-up . . . . . . . . . . . . . . . . . . . . . 61

31. Reflection mechanism . . . . . . . . . . . . . . . . . . . 65

32. Total internal reflection . . . . . . . . . . . . . . . . . . . . . 66

33. Wave vector decomposed into further components . . . . . . . . . . . . 69

34. Valence and Conduction bands . . . . . . . . . . . . . . . . 73

35. Silicon-on-Insulater (SOI) substrate . . . . . . . . . . . . . 75

36. Sketch of a rib waveguide. . . . . . . . . . . . . . . . . 76

37. Structure of strip nano-waveguide . . . . . . . . . . . . . . . 77

38. Graphical representation . . . . . . . . . . . . . . . . . 78

39. nano-waveguide and laser beam diameter comparison . . . . . . . . . . 79

$40 . \quad$ Silicon inverse taper $\ldots \ldots \ldots$. . . . . . . . . . . . 80

41. Grating couplers with nano-waveguide $\ldots \ldots \ldots$. . . . . . . . . 81

42. Grating couplers attached to the nano-waveguide $\ldots \ldots \ldots$. . . . 81

43. Grating coupler with calculating of fill factor " $f$ ". . . . . . . . . . . . 83

44. SOI waveguide fabrication process $\ldots \ldots \ldots \ldots \ldots \ldots \ldots$

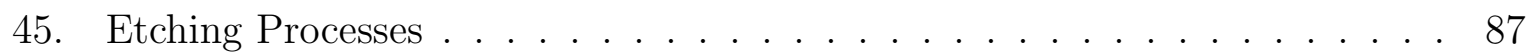

46. Lift off Processes . . . . . . . . . . . . . . . . . . . . . . 87

47. Atom Chip . . . . . . . . . . . . . . . . . . . 90

48. $\quad$ z-shaped double wire structure $\ldots \ldots \ldots \ldots \ldots$. . . . . . . . . 92

49. Simulation result for double z-wire $\ldots \ldots \ldots \ldots$. . . . . . . . . 93

50. $\quad$ Hyperfine transition structure of ${ }^{87} R b \ldots \ldots \ldots \ldots \ldots \ldots$

$51 . \quad$ Atom chip design $\ldots \ldots \ldots \ldots \ldots$. . . . . . . . . . . . 96

52. Silicon wafer structure for chip $\ldots \ldots \ldots \ldots \ldots$. . . . . . . . . 97

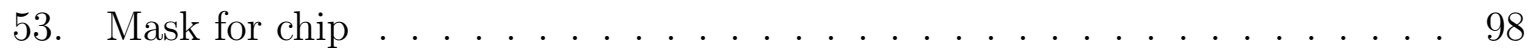

$54 . \quad$ Experimental set-up in KIT $\ldots \ldots \ldots \ldots \ldots$ 
55. SEM image of grating couplers . . . . . . . . . . . . . . . . . . . . . . 99

56. Detailed SEM image of the nano-waveguide . . . . . . . . . . . . . . . 100

$57 . \quad 2$ and $10 \mu \mathrm{m}$ SEM images . . . . . . . . . . . . . . . . . . . 100

58. Comparison plot between D1 and D5 coupler . . . . . . . . . . . . . . . . . 101

59. Glass cell . . . . . . . . . . . . . . . . . . . . . . . . . . . . 130

$60 . \quad$ Metal pipe . . . . . . . . . . . . . . . . . . . . . 131

61. Metal rod . . . . . . . . . . . . . . . . . . . . . . . . . . 132

62. Clamp 1 . . . . . . . . . . . . . . . . . . . . . 134

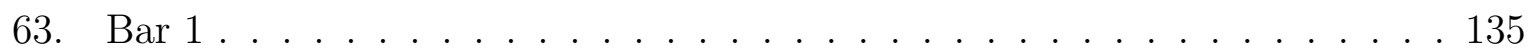

$64 . \quad$ Clamp 2 . . . . . . . . . . . . . . . . . . . . 136

$65 . \quad$ Bar 2 . . . . . . . . . . . . . . . . . . . . 137

$66 . \quad$ Clamp 3 . . . . . . . . . . . . . . . . . . . . . . . . . 138

$67 . \quad$ Bar $3 \ldots \ldots \ldots \ldots$

$68 . \quad$ Big clamp . . . . . . . . . . . . . . . . . . . . . . 140

$69 . \quad$ Bar $4 \ldots \ldots \ldots \ldots \ldots \ldots \ldots$ 


\section{List of Tables}

1. Specific values of the rectangular coils . . . . . . . . . . . . . . . 57

2. BIAS field for IBx, IBy and IBz . . . . . . . . . . . . . . . 61

3. $\quad$ Strip waveguide results from different Institutions . . . . . . . . . . . . . 77 


\section{Introduction}

\subsection{Background}

First idea of laser cooling technique was by Art Ashkin in his 1970 paper [1], in which he proposed the technique of interaction of single atoms with intense light. After four years, in 1974, Hänsch and Schawlow gave a practical idea of laser cooling i.e. the reduction of random thermal velocities using radiative force. In their paper they pointed out that the laser radiation is essentially isotropic, but confined to frequencies on the lower half of the Doppler-broadened absorption line of an atomic vapour, thus the gas can be cooled [2]. On other hand, continuing his research forward, Art Ashkin in 1978 coined the term optical trapping of how one can slow down the atomic beam of sodium using a laser beam tuned to an atomic resonance. In the same year, 1978, Wineland, Drullinger and Walls reported the first experimental evidence that bound absorbers can be cooled via radiation pressure, thereby fundamentally reducing both the first and second order Doppler effects. They published their results in the paper [3] that indicated the first laser cooling experiment, in which they cooled a cloud of Mg ions held in a Penning trap. Same year Neuhauser, Hohenstatt, Toschek and Dehmelt reported the laser cooling of trapped $\mathrm{Ba}^{+}$ions [4].

This is how the atom trap and laser cooling technique was demonstrated. Before this scientists considered lasers as tools for probing matter and not as tools for controlling it. Until the end of 1980's, physicists learned how to use lasers to cool atoms to temperatures barely above absolute zero. Until current times, different groups proposed different laser cooling techniques but by far most efficient, and still the most common method is Doppler cooling proposed by two groups in 1975. The first being David J. Wineland and Hans Georg Dehmelt [5] and the second being Theodor W. Hänsch and Arthur Leonard Schawlow [2]. In the end we see that basic concept is, and was, the same i.e. the force of laser light applied to atoms slow them down. 
In 1997 William D. Phillips, Steven Chu and Claude Cohen-Tannoudji received the Prize in Physics for developing methods to cool and trap atoms with laser light [6] [7] [8]. We shall discuss in later chapters of this thesis how this laser cooling or so called Doppler technique led to the creation of a new quantum form of matter called a Bose-Einstein condensate and further on to modern atomic clocks.

Partially our research area consists of design of atom chip, so it's worth here to give a slight taste of Integrated Quantum-optics and Optomechanics. The electronic circuit was discovered way back in 1800's and really changed the world [9]. At first electronic circuits consisted of resistors, transistors, capacitors, inductors and diodes, connected by conductive wires or traces through which electric current could flow. These combinations of components and wires allowed various simple and complex operations to be performed: signals could be amplified, computations could be performed, and data could be moved from one place to another [10].

On the other hand the demand of integrated chips are increasing day by day as they are applicable in all communication devices. In 1947 the invention of the solid state transistor allowed the miniaturization and integration of electronic circuits 11 [12 11. We all know that in electronic circuits, the main source of transferring the data is electron. Electronic devices have long since been used for data communication and transfer but nowadays this had to change because growing demand for smaller size, lower power and lower cost are increasing. Now scientists prominently use photons for communication, especially in silicon on insulator wafers and trying to send the data optically rather than on electronic circuits 13] 14].

\subsection{About this thesis}

The goal of the present work is to establish a new experimental apparatus for the study of light-matter interactions. In this apparatus a cold cloud of Rubidium atoms will be realized by means of a standard atom chip setup. The functionality of the atom chip will 
be enhanced by embedding photonic structures onto its surface and the Rubidium atoms will be steered by means of suitable magnetic fields close to those photonic structures. To allow for rapid exchange of the atom chip, such as for testing improved photonic structures or to implement additional electrical components, the whole system is to be equipped with a load-lock system that allows a replacement of the chip without breaking the vacuum of the main part of the instrument.

This thesis is divided in two parts. Part 1 reveals the theory and experiment work done in LENS laboratory Florence University and part 2 is about the theory and experiment done in KIT (Karlsruhe Institute of Technology) University.

Part 1 is based on two chapters, Chapter 2 and Chapter 3 . Chapter 2 describes the theory part of cold atoms. In Chapter 2 we start with the theory of atomic properties in rubidium $(\mathrm{Rb})$ atoms. We briefly describe the atom light interaction, then we address the $\mathrm{Rb}$ hyperfine structure in detail. Theoretically we described the laser cooling technique and how Rb atoms trap through laser cooling technique. We tried to pin point the Magnetic Optical Trap (MOT) through an experiment. We used this experiment to try to understand the MOT experiment, in this way we go in depth and understand very basics of MOT experiment. In the end of Chapter 2 we differentiate the mirror MOT and ordinary MOT. Chapter 3 discloses our Magnetic Optical Trap (MOT) set up which we build up at LENS. The design, calculation and simulation of the MOT set up are described in detail. We start with Ultra High Vacuum (UHV) chamber and a step by step description of every part of the set up. We also described the optical system for atom trapping and the anti-Helmholtz and BIAS coils.

Part 2 of the thesis is based on the theory and experimental work for atom chip. It's worth mentioning here that our purpose is to load cold atoms near to photonic structure that are situated few hundred microns apart. The photonic structure is exactly at the center of Atom Chip. The surface of the Atom Chip will be placed as near as possible of the MOT, which is due to the distance necessary to transfer cold atoms from the MOT to the z-wire trap on the chip. Chapter 4 tells us the in depth concept of silicon photonics, 
grating couplers and waveguides. In Chapter 5 we go through the design, parameters, simulation and fabrication of our atom chip. We discuss in detail why we used the certain parameters of atom chip and why we design and fabricate the chip.

Last, but not least, in Chapter 6 we described applications, conclusion and future work. 


\section{Theory of Laser Cooling}

\subsection{Introduction}

We assume that we are all familiar with the concept of quantum mechanics governing atomics structure and photons transferring momentum to atoms which allows light to exert a force on atoms. The velocity and position dependence of this force can be used to cool and trap neutral atoms.

In 1853, A. J. Ångström achieved a landmark by discovering the spectral line of atomic hydrogen [16]. In continuation of his research, A. A. Michelson noticed a finer structure within these spectral lines [17] [18].

Neil's Bohr's idea of an electron in certain stationary states produced a solar system like model for atom. He visualized that atom consisted of nucleus and electron, where electron orbits the nucleus in quantized energy levels. He labeled each energy level with the integer $n$ and called it principal quantum number, between 0 to $n-1$. We further have the quantum number $l$ describing game orbital momentum of the electron orbiting the nucleus. The projection of the orbital angular momentum along a coordinate axis describes the magnetic quantum number $m_{l}$ which is an integer between $-l$ and $l$ [19] [20]. The concept of electron spin, $s=1 / 2$, was introduced by Uhlenbeck and Goudsmit in order to explain the wavelengths and intensities of spectral lines. Dirac theorized a proton and neutron spin in a theory which unified quantum mechanics and special relativity. The electron spin's projection along a coordinate axis is denoted by the letter $s$, either $+1 / 2$ or $-1 / 2$. The nuclear spin is labeled with the letter $I$ and varies depending on the nucleus 21]. Both Bohr and Planck theorized that an electron could change energy levels by emitting or absorbing a photon of corresponding energy but only certain energy level changes are allowable due to selection rules which govern electron transitions. An electric dipole transition must have $\Delta l=1, \Delta m_{l}=-1,0,+1$, and $\Delta m_{s}=0[22$. The interplay of these ideas gives us insight into the light (photon)-atom interaction and brings us forward 
to laser and dipole cooling.

This chapter describe mostly the theory part of atom-light interaction. Rubidium atom will be the part of our experiment so we begin with the description of Rubidium ( $\mathrm{Rb}$ ) atom. We also describe the laser cooling technique and trapping of Rb atom. We discuss the Magnetic optical trap (MOT) set up experiment for understand the basics of cold atoms. We didn't discuss about our Atom Chip in this and next chapter, as we leave the discussion of Atom Chip for the next part in detail.

\subsection{Properties of Rubidium ( $R \mathbf{b})$}

The discovery of Rubidium ( $\mathrm{Rb}$ ) atom was made by German chemist Robert Bunsen and Gustav Kirchhoff in 1861 [23] [24]. They developed a new technique called flame spectroscopy [25]. Rubidium has many application in chemicals and electronics devices but we are going to focus particularly on vaporized ${ }^{87} \mathrm{Rb} .{ }^{87} \mathrm{Rb}$ is one of the most commonly used atomic species utilized for laser cooling and Bose-Einstein condensation. ${ }^{87} \mathrm{Rb}$ applications are favored by the availability of inexpensive diode laser light at the relevant wavelength, and the moderate temperatures required to obtain substantial vapor pressures 26] 27]. 28 .

When we study atoms in gas, they are moving very rapidly and it's very hard for us to take any kind of measurements. The Doppler shift and the relative time dilation causes displacement and broadening of the spectral lines of thermal atoms, which have a wide spread of velocities. High velocities of atoms also have a drawback of limited observation times. The moving speed of atoms and molecules are $300 \mathrm{~m} / \mathrm{s}$ at room temperature that is equal to the speed of sound and it's very difficult to investigate with high precision the structure of atoms moving at this speed. One can reduced this speed to the thermal velocity of Nitrogen $\left(N_{2}\right)$ by refrigerating it which gives the speed of $150 \mathrm{~m} / \mathrm{s}$ at $77 \mathrm{~K}$, which puts $N_{2}$ into liquid state. Helium $(\mathrm{He})$ atoms have $90 \mathrm{~m} / \mathrm{s}$ speed at $4 \mathrm{~K}$. But scientist wants to be more precise and want to bring the atoms in a gas to velocities of less than 1 
$\mathrm{m} / \mathrm{s}$ with almost the temperature in micro Kelvin. Scientists started the experiments for cooling both neutral atoms and ions for the motivation of atomic clocks. The discovery led to the study of some remarkable new phenomena like collisions in cooled atoms and of quantum collective behavior 29] [30].

\subsubsection{Rubidium (Rb) Hyperfine Structure}

The Rb atom has one valence electron, thus making its energy level structure relatively simple as compared to other atoms in periodic table. The other main reason for using $\mathrm{Rb}$ atoms is that the energy difference between the ground state $|g\rangle$ and the excited state $|e\rangle$ is

of the order of $1.5 \mathrm{eV}$, making it easily accessible high resolution atomic spectroscopy [31. Last but not least, alkali metals in general have a vapor pressure which increases by several orders of magnitude when they are heated up (e.g. sublimation temperature for Rubidium $(\mathrm{Rb})$ is $39.31^{\circ} \mathrm{C}$, Potassium $(\mathrm{K}) 63.38^{\circ} \mathrm{C}$, and Cesium $(\mathrm{Ce}) 28.44{ }^{\circ} \mathrm{C}$ ) 32 .

We used Rubidium (Rb) atoms in our experiment and natural Rubidium comes in two isotopes, ${ }^{85} \mathrm{Rb}$ and ${ }^{87} \mathrm{Rb}$ respectively with the fractional abundances of 0.7217 and 0.2783 , atomic masses 84.912 and 86.909 and with the atomic number 37. In its lowest (ground state) configuration it has one electron outside an inert gas (argon) core and its ground state configuration described with the notation $1 s^{2} 2 s^{2} 2 p^{6} 3 s^{2} 3 p^{6} 3 d^{10} 4 s^{2} 4 p^{6} 5 s^{1}$, the integers 1 through 5 describe the principal quantum numbers $n$. The letters $s, p$ and $d$ specify orbital angular momentum quantum numbers $l$ as 0,1 and 2 respectively. The superscripts indicate the number of electrons with those values of $n$ and $l$. The next higher energy configuration has the $5 s$ valence electron promoted to a $5 p$ orbital with no change to the description of the remaining 36 inner electrons 33.

\subsubsection{Fine structure}

Fine structure defines the splitting of spectral lines of any atoms. This splitting occurs in atoms because of electron spin and the relativistic corrections to the non-relativistic 
Schrödinger equation [34]. There can be several fine structure energy levels within a configuration that differ in the energy associated with the coulomb and spin orbit interactions. The Coulomb interaction is given by $k q_{1} q_{2} / r^{2}$ where, $k=$ Coulomb's constant $=\frac{1}{4 \pi \epsilon_{\circ}}$. This electrostatic potential energy exists between each pair of electrons and between each electron and the nucleus. The spin orbit interaction is associated with the orientation energy $-\vec{\mu} \cdot \vec{B}$ of the magnetic dipole moment $\mu$ of each electron in the internal magnetic field $\mathrm{B}$ of the atom 35 .

Russell-Saunders $(L-R)$ coupling scheme described the strength and the form of these two interactions in Rubidium atoms for the accuracy of energy levels. The coupling introduces new angular momentum quantum numbers $L, S$ and $J . L$ and $S$ are the total orbital and spin angular momentum quantum numbers respectively which are the sum of the orbital and spin quantum numbers $l$ and $s$ respectively. $J$ is the sum total of both these angular momentum quantum numbers and is given by $(J=L \pm S)[36]$.

The values for $L, S$ and $J$ are specified in a notation ${ }^{(2 S+1)} L J$. The value of $2 S+1$ is called the multiplicity and is thus 1 for $S=0$ (singlet) and 2 for $S=\frac{1}{2}$ (doublet). The letters $S, P$ and $D$ are used for $L$ and correspond to $L=0,1,2$. For Rb configurations with only one valence electron, there is only one allowed value for $L$ and $S$. For 5 s configuration, $l=0$ and $s=\frac{1}{2}$ and therefore $L=\sum l=0$ and $S=\sum s=\frac{1}{2}$. So, the only possible value for $J=\frac{1}{2}$ and the fine structure can be labeled as ${ }^{2} \mathrm{~S} \frac{1}{2}$. The electron will jump to $5 p$ level upon excitation. So, for the next higher level configuration, $l=1$ and $s=\frac{1}{2}$ and therefore, $L=1$ and $S=\frac{1}{2}$. The value of $J$ is from $L-S=\frac{1}{2}$ to $L+S=\frac{3}{2}$. There are two fine structure states labeled as ${ }^{2} P_{\frac{1}{2}}$ and ${ }^{2} P_{\frac{3}{2}}$. We are interested in the transition from ${ }_{5}^{2} S_{\frac{1}{2}}$ to ${ }_{5}^{2} P_{\frac{3}{2}}$ having a difference of $780 \mathrm{~nm}$ between these levels [37], that is shown in Figure 1 .

\subsubsection{Hyperfine Levels}

The fine structure levels further divide into even finer sets of levels known as hyperfine levels, which are differing in the orientation energy associated with the nuclear magnetic 


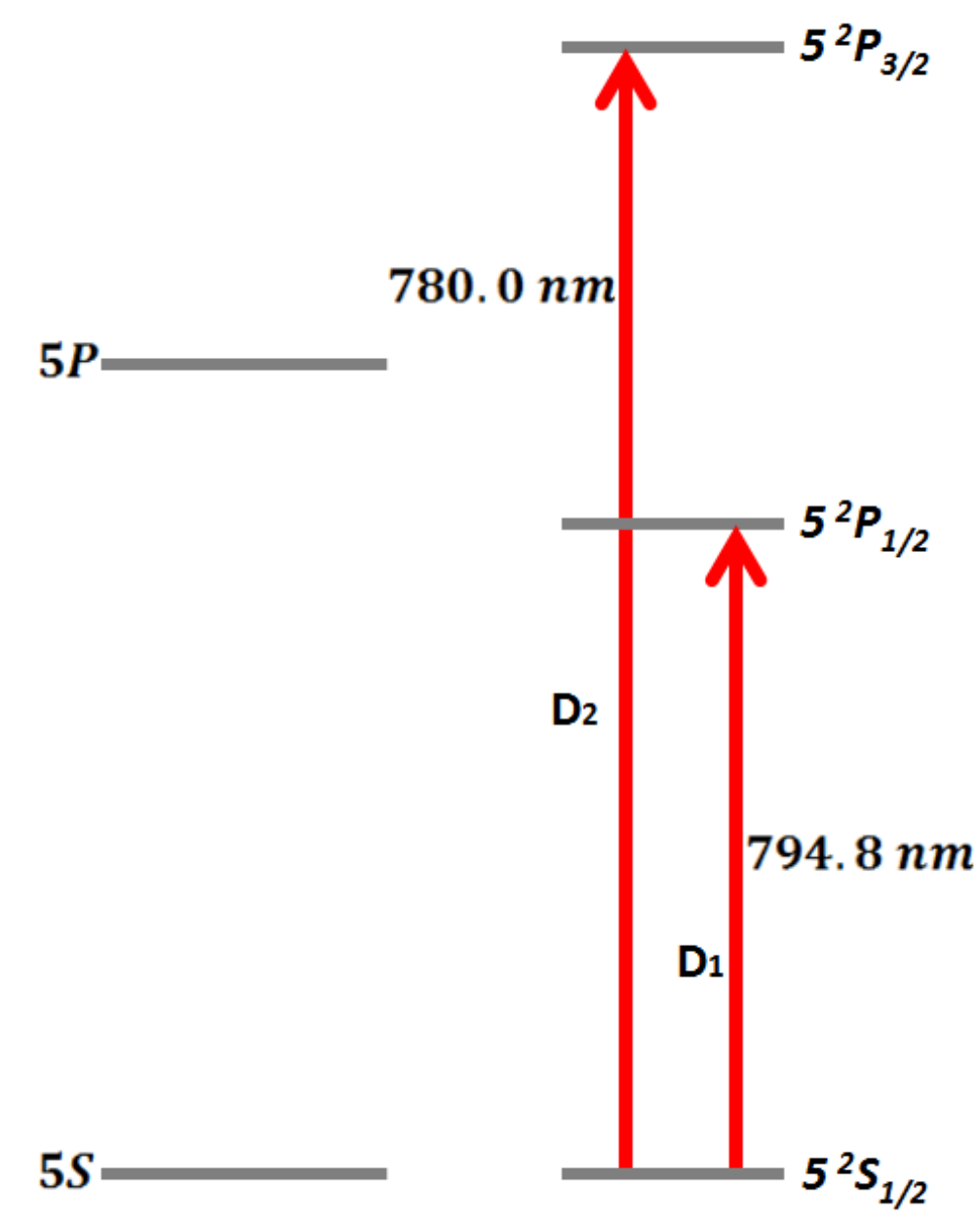

Figure 1: Figure showing the Rubidium $(\mathrm{Rb})$ fine level structure, lines are known as D1 and D2 (Figure regenerated from [38]). 
moment. These finer structures will serves as a tool of our experiment and will play very important role for maneuvering the atoms. The nuclear magnetic moment is much smaller than the electron magnetic and so the hyperfine splittings are small. The nuclear magnetic moment is proportional to the spin angular momentum I of the nucleus, whose magnitude is described by the quantum number $I$. The hyperfine energy levels depend on the total angular momentum $F$ of the atom and is equal to the sum of the total electron angular momentum $J$ and the nuclear spin angular momentum $I(F=J+I)[39]$. This gives us the possible quantum numbers $J-I, J-I+1, \ldots, J+I-1, J+I$. Here it is worth mentioning that the value of $I$ depends on the isotope, i.e. for ${ }^{87} \mathrm{Rb}$ it is $\frac{3}{2}$ and for ${ }^{85} \mathrm{Rb}$ it is $\frac{5}{2}$. The value of $J$ on ground state is $\frac{1}{2}$ and for excited state is $\frac{3}{2}$ for transition between $5 S_{\frac{1}{2}}$ to $5 P_{\frac{3}{2}}$. This leads to two hyperfine levels for $S_{\frac{1}{2}}\left(I-\frac{1}{2}\right.$ to $\left.I+\frac{1}{2}\right)$ and four hyperfine levels for $P_{\frac{3}{2}}\left(I-\frac{3}{2}\right.$ to $\left.I+\frac{3}{2}\right)$. The selection rules for electric dipole transitions are given by

$$
\begin{array}{r}
\Delta F=0 \text { or } \pm 1(\text { but not } 0 \rightarrow 0) \\
\Delta J=0 \text { or } \pm 1 \\
\Delta S=0 \\
\Delta L= \pm 1
\end{array}
$$

As seen from the Figure 2, there are two groups of three transitions possible from each of the two hyperfine splittings of ground state. Since the difference between these two levels is large, these groups will be well separated from each other. But within each group, they will be closely spaced in energy [40].

\subsection{Atom-Light Interaction}

Before the invention of Laser, the property of light was first observed by a very small transverse deflection $\left(3 \times 10^{-5} \mathrm{rad}\right)$ in a sodium atomic beam that exposed to light from a resonance lamp. After this observation, one can understand that light beam carries 


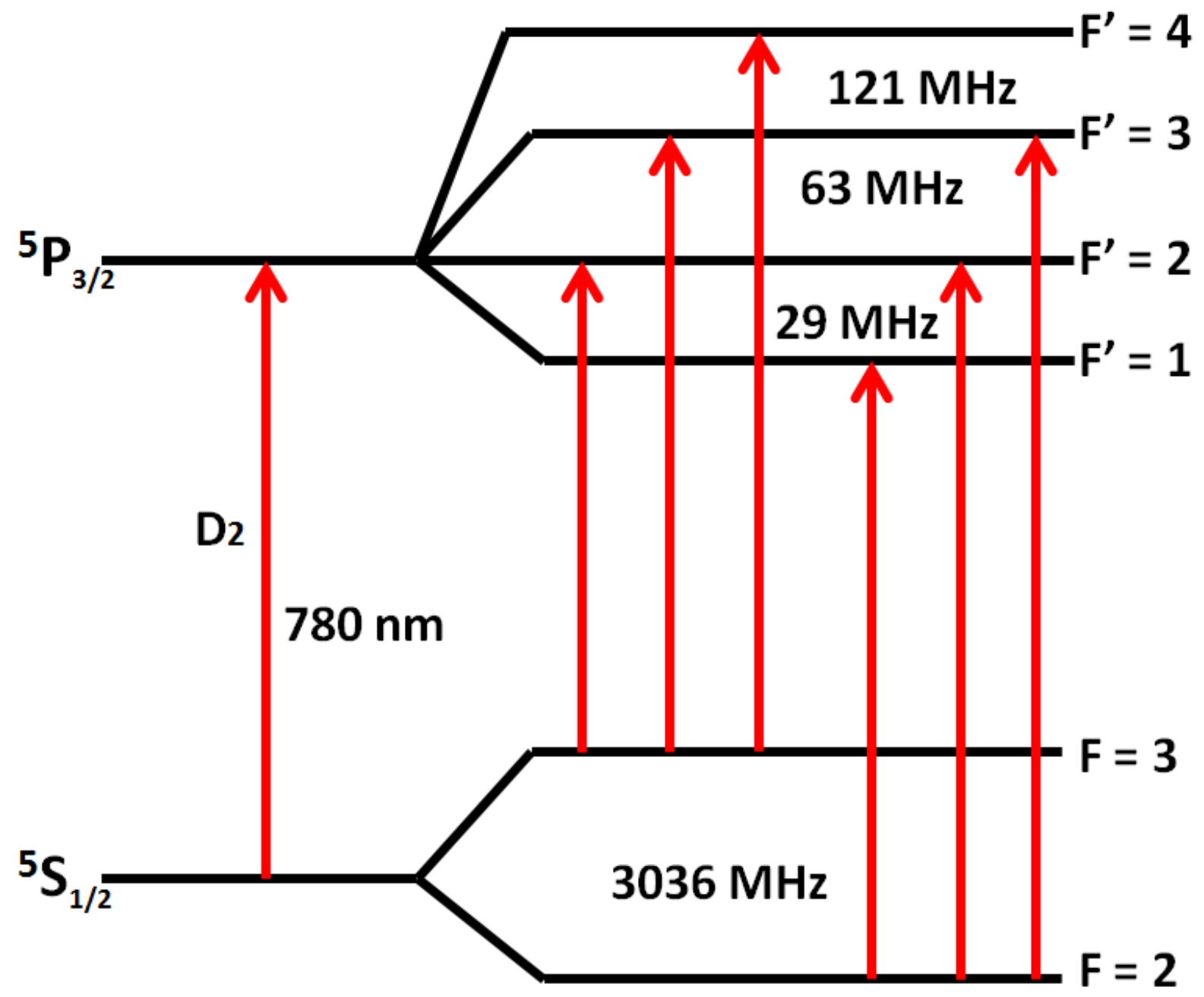

Figure 2: Rubidium $\left({ }^{85} \mathrm{Rb}\right)$ hyperfine splitting levels, where $\mathrm{MHz}=$ Mega-Hertz (Figure regenerated from [41]). 
a momentum and the scattering of light by an object produces a force on that object. After the invention of Laser it's very easy to take these kinds of observations, because the strength of the force in Laser light is greatly enhanced by the use of intense and highly directional light fields. The force on an atom by light can be divided into two effects [42], as we will see in the following:

\subsubsection{Optical dipole force (Conservative)}

The convenient way to understand the dipole force is by considering the light as a classical wave. It is a time-averaged force that stems from the interaction of the transition dipole, induced by the oscillating electric field of the light, with the gradient of the electric field amplitude. This is the force which can trap an atom in the focus of a laser beam. It is conservative and therefore can be represented by a potential. Focusing the light beam controls the magnitude of this gradient, and detuning the optical frequency below or above the atomic transition controls the sign of the force acting on the atom. Tuning the light below resonance attracts the atom to the center of the light beam while tuning above resonance repels it. The dipole force is a stimulated process in which no net exchange of energy between the field and the atom takes place. Photons are absorbed from one mode and reappear by stimulated emission in another. Momentum conservation requires that the change of photon propagation direction from initial to final mode imparts net recoil to the atom 43 .

\subsubsection{Spontaneous Force (Dissipative)}

In two level states when an atom absorbs and emits photon, spontaneous force arises due to scattering of light by an atom. The resonant scattering cross section is given by:

$$
\sigma_{0} a=\frac{g_{1}}{g_{2}} \frac{\pi \lambda_{0}^{2}}{2}
$$




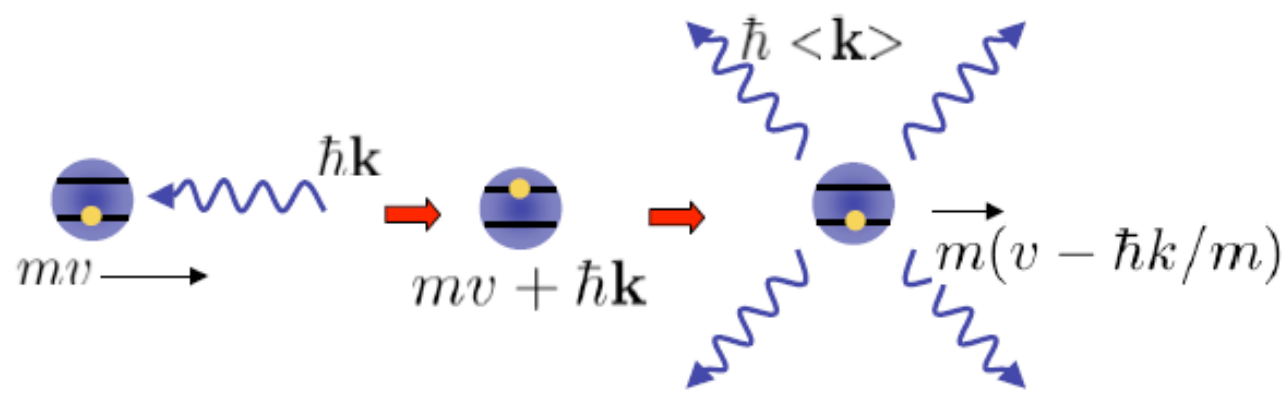

Figure 3: On Left: atom has a momentum $p=m v$ encounter a photon (momentum $=\hbar k$ ) that is traveling from the opposite direction. At Center: atom absorb the photon and come in excited state (now momentum of the atom is equal to the sum of the initial atom and photon momenta). On Left: spontaneously emitting a photon in a random direction, now atom again is in ground state with a new velocity $\left(v^{\prime}=m v-\hbar k\right)[45]$.

Where;

$\lambda_{0}^{2}=$ on-resonant wavelength

$g_{1}=g_{2}=$ constant

$\sigma_{0} a=$ resonant scattering cross section (we know that in the visible region of the electromagnetic spectrum the wavelengths of light is hundreds of nanometers, so resonant scattering cross sections become quite large $\sim 10^{-9} \mathrm{~cm}^{2}$ ).

Each photon absorbed transfers a quantum of momentum $\left(\frac{k h}{2 \pi}\right.$, where $h=$ Planck's $^{\prime}$ constant) to the atom in the direction of propagation. The spontaneous emission following the absorption occurs in random direction, and over at least 1000 absorption emissions it averages to 0 [44]. The net spontaneous force is shown in Figure 3

\subsection{Laser Cooling}

Whenever the term laser cooling come in our minds, the first question that arises is, can lasers really cool? Because we normally think that laser can make things hot, it can cut pieces off metal or fire rockets out of the sky but Steven Chu [46], Claude Cohen Tannoudjei 47] and William D. Philips [48 received the Noble Prize in 1997 for their development of methods to cool and trap atoms with laser light. Laser cooling has different techniques but the basic principle applied is same in every technique in which atomic, 


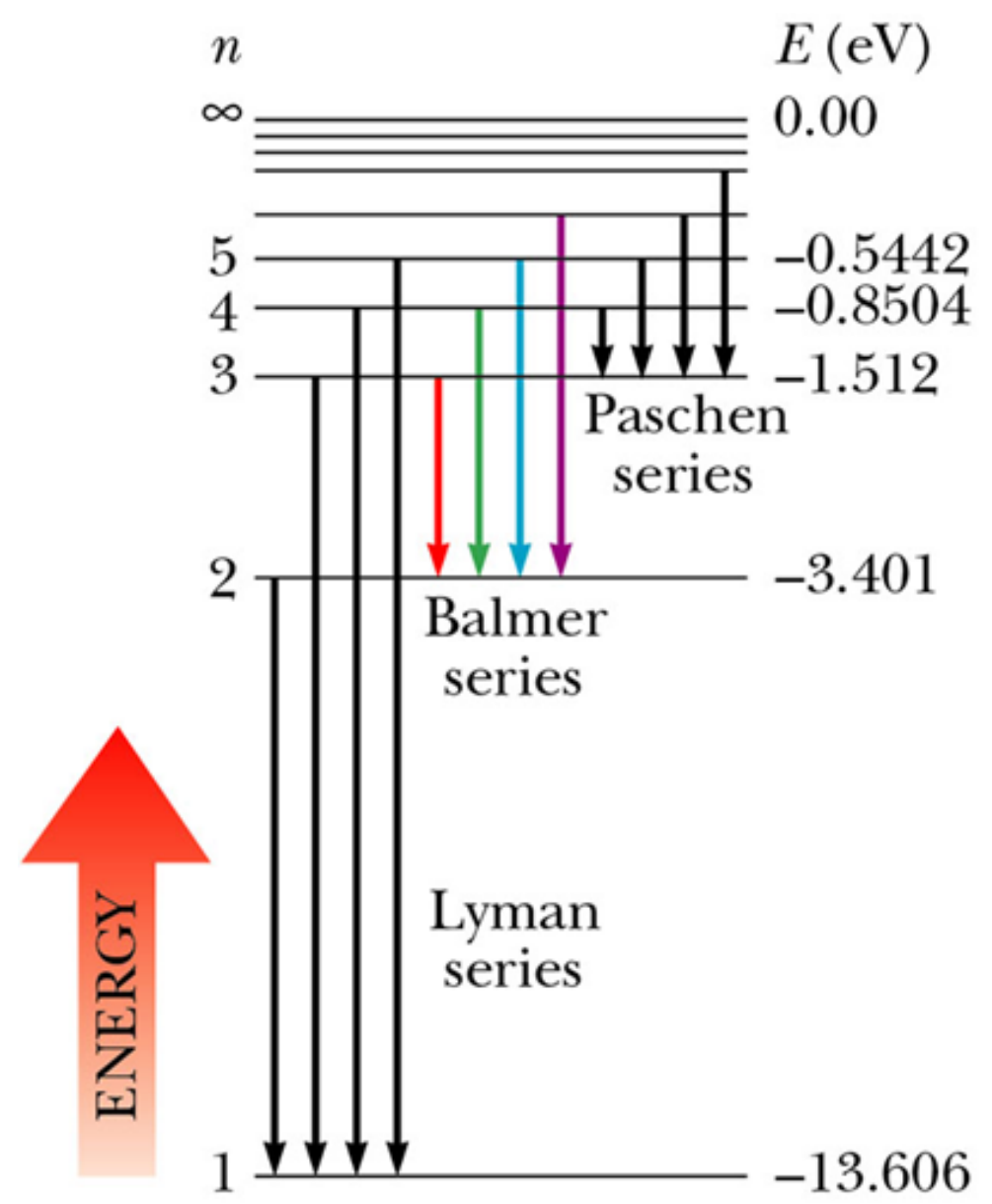

Figure 4: Hydrogen energy level structure with, lowest energy state (ground state) correspond to $\mathrm{n}=1$ with Energy $=-13.6 \mathrm{eV}$, the next energy level has an Energy $=-3.40 \mathrm{eV}$. The uppermost level corresponds to $\mathrm{E}=0$ and $\mathrm{n} \longrightarrow \infty$ 49.

molecular and ion samples are cooled down to near absolute zero through the interaction of one or more laser fields. When the atom absorbs and re-emits a photon its momentum changes, the momentum of atom is directly proportional to its kinetic energy. For a broader picture and to understand deeply, we consider a simple Hydrogen atom that has energy levels in ground state $(n=1)$ and excited state $(n=2,3, \ldots)$ as shown in Figure 4.

Figure 4 shows the energy level diagram for hydrogen atoms in the presence of strong electric and magnetic fields that split the energy levels into further hyperfine structure, where energy is shown on the vertical axis as increasing energy levels. Quantum mechanics tells us that the atom occupies a fixed energy level [50].

Quantum mechanics also explains that photons are particles of light that carry energy 


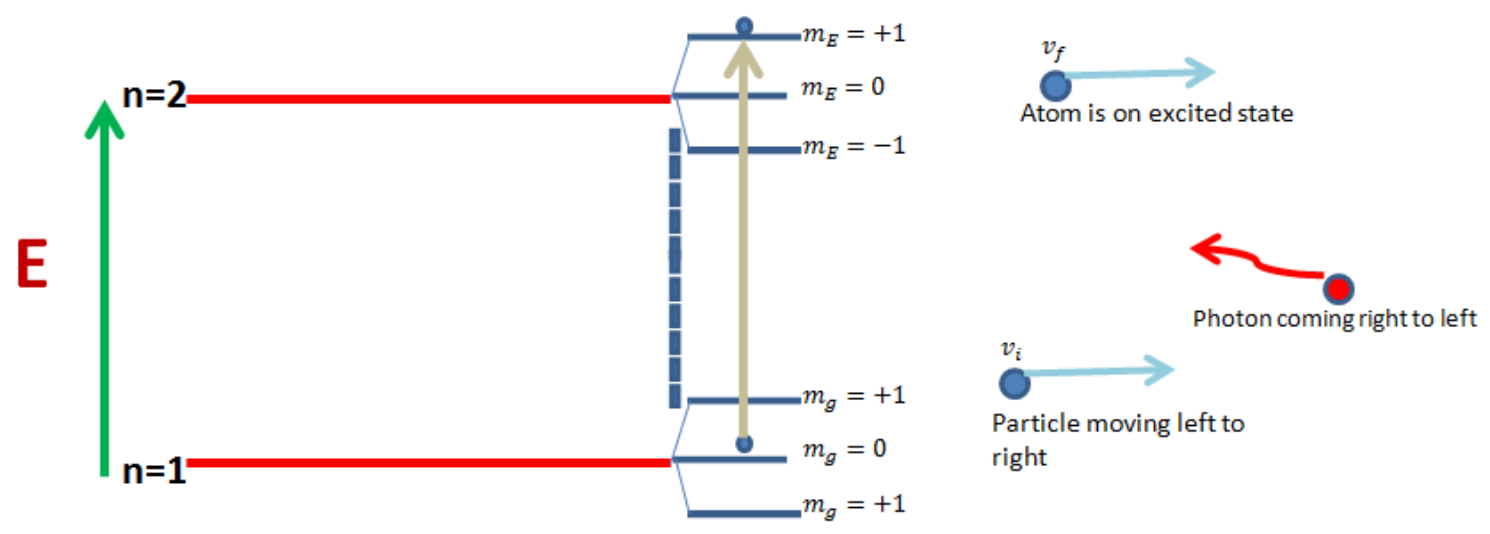

Figure 5: Left: Atom in Hyper splitting from $m_{g}=0$ to $m_{E}=+1$ state, Right: Particle moving left to right to meet photon

and momentum that is given by the relationship

$$
E=h f
$$

Where,

$f=$ Photon frequency

$h=$ Planck constant

$E=$ Photon energy

$$
p=\frac{h}{2 \pi} k
$$

Where,

$k=$ Constant term

Now we consider an atom in $m_{g}=0$ state with velocity $v_{i}$, which is moving from left to right, at the same time photon is coming from right to left and hits the atom as shown in Figure 5 .

This photon has an energy of $E_{\gamma}$ to bring the atom from $m_{g}=0$ to $m_{E}=+1$ state with velocity $v_{f}$. Momentum conservation tells us that the final momentum of an atom is the initial momentum plus the momentum carried by the photon, which is in the 


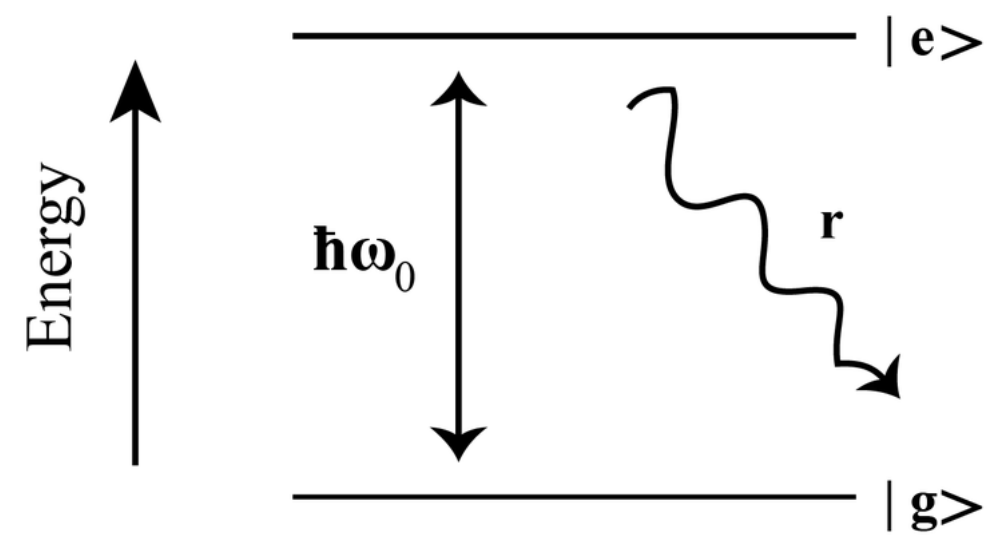

Figure 6: Energy level diagram of two level atom, The difference between both level is given by $\hbar \omega$ with the rabi frequency $\omega_{0} 53$

opposite direction. It means that the final momentum of an atom is actually reduced which concludes that final velocity $\left(v_{f}\right)$ is less than the initial velocity $\left(v_{i}\right)$. The excited atom will spontaneously decay back to the ground state, however, since the spontaneous photons are emitted isotropically on average no net momentum is acquired by the atom in this process. In this way photons collide several times to slow down the atom. We just have to put the laser in right frequency. The atomic temperature can decrease to a few micro Kelvin. This is the basic idea behind laser cooling. Laser cooling has several examples but still the most common and important method is Doppler cooling [51], which we discuss in a later section, in Magneto Optical Trap (MOT) [52.

\subsection{Magneto Optical trapping (MOT)}

Magneto Optical trap (MOT) is very common these days in modern atomic physics. It produces extremely cold gases of atoms. To understand MOT in a better way we have to start with light-atom interaction. For simplicity let's start with two level atom that contains a ground state $|g\rangle$ and excited $|e\rangle$ state. This atom doesn't have the complexity of an atom like Rb, two level atom diagram is shown in Figure 6 .

The ground state $|g\rangle$ is connected by optical absorption to an excited state $|e\rangle$. For absorption, emission and atom-light interaction we must know the properties and tools of 
this two level atom. The resonance frequency for driving the transition between $|g\rangle$ and $|e\rangle$ state is given by following expression:

$$
E_{o}=\frac{h}{2 \pi} \omega_{o}
$$

Where,

$E_{o}=$ Energy separating ground and excited state

$\omega_{o}=$ Frequency

For two level atom ground $|g\rangle$ and excited $|e\rangle$ state optical transition is described by a line-width $(\Gamma)$ that relates to how the excited state $|e\rangle$ will emit a photon into the empty vacuum and is given by:

$$
\begin{array}{r}
\text { Line-width: } \Gamma\left(\frac{\mathrm{rad}}{\mathrm{s}}\right), \frac{\Gamma}{2 \pi}(H z) \\
\text { Lifetime: } \frac{1}{\Gamma}
\end{array}
$$

Line-width $(\Gamma)$ is related to the lifetime $|e\rangle$ of an atom in the excited state, it means how long atom will be reside in the excited state $|e\rangle$ before typically a photon is emitted into the vacuum. The monochromatic plane wave laser frequency is " $\omega$ ", that is detuned by $\delta=\omega-\omega_{o}$ (Detuning between the frequency of atom and its resonance frequency). Monochromatic wave has the propagation vector $k$, also known as wave vector that is $2 \pi / \lambda$, the strength of the optical field, known as Rabi frequency, that tells us what will be the oscillation frequency of the atom as it is driven from the ground state $|g\rangle$ to excited state $|e\rangle$. If there is no spontaneous emission at all then it's just a coherent process.

$$
\text { Rabi frequency }=\Omega=\frac{\vec{E} \cdot \vec{d}}{\hbar}
$$

Where,

$\vec{E}=$ Laser electric field

$\vec{d}=$ Dipole moment of atom 


$$
\text { Saturation Parameters }=S_{o}=\frac{I}{I_{s a t}}=\frac{2 \Omega^{2}}{\Gamma^{2}}
$$

Saturation Intensity $I_{s}$ tells us how strongly the atom is interacting with light when light is incident on atom [54] [55].

For the MOT (magneto optical trap) we have to use a magnetic field gradient that will be set up in such a way that atom moving away from the center of the trap is Zeeman shifted (described in section 2.7.1) onto resonance with a laser beam pushing it back. For simplicity, we take the example of one-dimensional model in which an atom has a allowed transition of $F=0 \longrightarrow F^{\prime}=1$. We require a field that is linearly along the direction $(\mathrm{z})$ of propagation beam, that is given as, $B=|B|=\beta|z|$, where $\beta=$ gradient. The Zeeman shift is given by:

$$
\Delta E=\mu_{B} g_{F} m_{F} B
$$

Where,

$\mu_{B}=$ Bohr magneton

$g_{F}=$ Landé g-factor

$z=0=$ Zero of the field located

Figure 7 shows the energy level shifts with a pair of counter propagation laser beams having frequency $\omega_{L}$ red detuned from the transition frequency $\omega_{o}$. Figure 7 also shows that both beams are right hand circularly polarized but when both beams are propagating against (along) the quantization axis then they drive $\sigma^{-}\left(\sigma^{+}\right)$transition (by the direction of magnetic field). $m_{F}=-1$ showing that the combination of re detuned and Zeeman shift of the beams is always closest to the resonance with the laser light. Accordingly, atoms will be more scatter light from the incoming beam and pushed towards the center of trap, $z=0[56]$.

Until now our discussion restricted to 1-D MOT. For 3-D MOT, three orthogonal pairs of counter-propagating beams will be used and a quadrupole field produced by two coils in the anti-Helmholtz configuration, e.g. $B=b(x, y,-2 z)$. Two coils in the anti-Helmholtz 


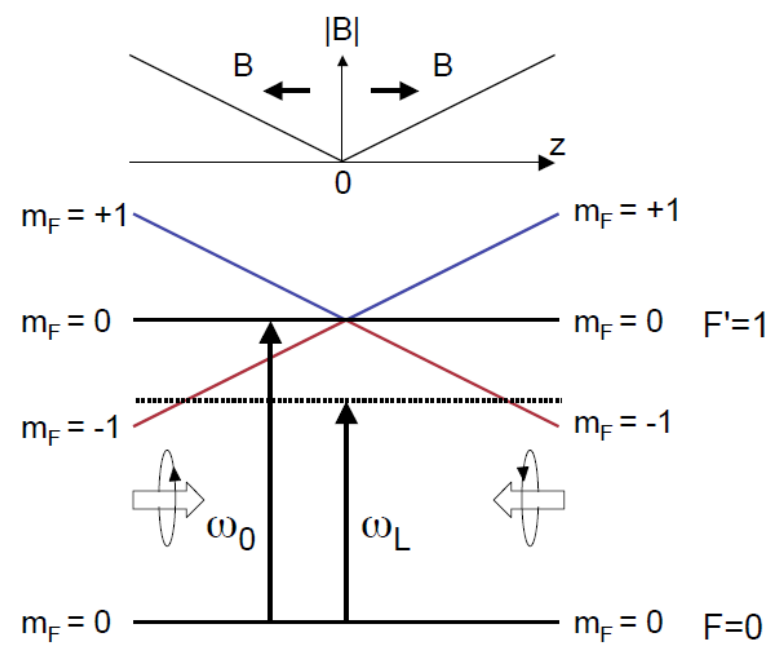

Figure 7: 1-D MOT having a transition from $F=0 \longrightarrow F^{\prime}=1$, the direction of the magnetic field $B$ is changes at the center. Polarization of both beams (left and right) drive $\sigma^{-}$ transition relative to the magnetic field towards the trap center [56].

configuration will be the discussed in next Chapter and that is also the subject of our experiment.

\subsection{Optical Molasses}

Optical Molasses is a technique of laser cooling in which neutral atoms can cool down to the temperature that will be colder then Magneto Optical trap (MOT). In optical molasses three pairs of counter-propagating circularly polarized laser beams intersect in the region where the atoms are present. That is shown in Figure 14. The laser light will only interact with those atoms that have certain frequencies, indicated by the peak of the curve that is called resonance peak. When the laser light finds correct atoms at right resonance, they cancel each other out and the atoms feel no net force. Doppler shifting occurs due to the motion of the atoms which means each atom will see a different frequency depending on which direction its moving. By making the frequency little lower, that resonance frequency will cause atoms moving towards the laser to see the resonance frequency and making the frequency little higher will cause atoms in the lasers to speed up. So the laser is tuned to a frequency slightly lower than the frequency which allows the atoms to easily absorb photons. The theoretical limit of lowest possible temperature 
also know as Doppler limit is given by 57

$$
T_{d}=\frac{h \Gamma}{4 \pi k_{b}} k
$$

Where,

$\Gamma=$ Natural line-width of atomic transition

$\mathrm{h}=$ Planck's constant

$k_{b}=$ Boltzman Constant

Optical molasses can cool the atoms until some $\mu \mathrm{K}$, that can cool down neutral atoms to temperature colder then MOT [58] [59].

\subsection{Mechanical effects that make MOT really work}

\subsubsection{Doppler Cooling}

Let's consider the situation where two level atoms seek incident of two laser beams. First

one with wave vector $\vec{k}_{1}$ and the next one with wave vector $\vec{k}_{2}$. This time the atom is not in stationary position rather it's moving and in the rest frame of this atom the frequencies of the different laser beams will be shifted according to the first order Doppler shift. As far as the atom is concerned the detuning of the first laser is given by

$$
\delta_{1}=\delta-\left(\overrightarrow{k_{1}} \cdot \vec{v}\right)
$$

In above equation, negative sign shows that if laser beam is heading against the direction of motion of the atom, then the frequency of the laser light will be seen to be higher e.g. "blue shifted" that will increase the detuning " $\delta_{1}$ ". Similarly the detuning of second laser beam is given as

$$
\delta_{2}=\delta-\left(\overrightarrow{k_{2}} \cdot \vec{v}\right)
$$


Now we consider the situation where the motion is in one direction and the laser beam has equal intensity but has opposite direction.

$$
\text { 1-dim: equal } I, \omega:\left|\overrightarrow{k_{1}}\right|=\left(\overrightarrow{k_{2}}\right)
$$

In that case, the scattering rate is given by the atom for light that is coming out of laser beam 1 and laser beam 2 .

$$
\Gamma_{s_{1,2}}=\frac{\Gamma}{2} \frac{S_{o}}{1+S_{o}+\left(\frac{\Gamma}{2}(\delta \pm(k v))\right)^{2}}
$$

When this light scattering strikes bunch of atoms in vacuum chamber then radiation scattering force for the one beam is,

$$
\Delta \vec{P}_{\text {atom }}=\hbar \vec{k}-\hbar \vec{k}_{\text {out }}
$$

Every time an atom absorbs a photon from the laser beam it gains one unit of the photon momentum and then afterward the photon is re-scattered away from the atom in a random direction and it also changes the momentum of atom. On average, the momentum of photon is,

$$
<\Delta \vec{P}>=\hbar \vec{k}
$$

Now if we take the scattering rates $\left(\Gamma_{s}\right)$ and turn them into forces, equal to the rate of change of atomic momentum, then atomic radiation force is given by,

$$
<F_{\text {rad }}>=\hbar k\left(\Gamma_{s_{1}}-\Gamma_{s_{2}}\right)
$$

$F_{\text {rad }}$ also tell us about the net force of atom when two beams are incident upon it. It is given by the difference of laser beam heading in one direction and laser beam heading towards other e.g. $\left(\Gamma_{s_{1}}-\Gamma_{s_{2}}\right)$. We can use this radiation force to cool down the atoms [52]. The velocity dependent force exerted on an atom for typical parameters of a MOT is 


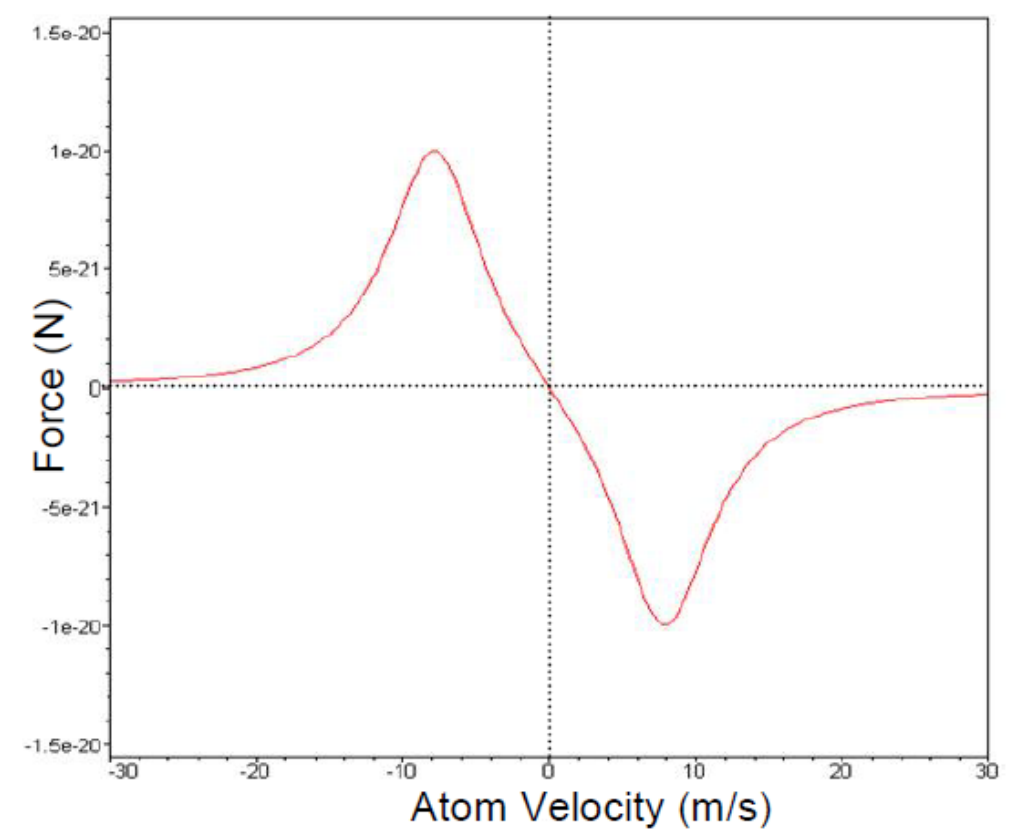

Figure 8: Graph showing Force (y-axis) calculated on an atom from Doppler cooling as a function of atom velocity (x-axis). We assume e.g. $\mathrm{I} / I_{\text {sat }}=2$ and $\delta=10 \mathrm{MHz}$ typical MOT load detuning. We can see force starts out from $0 \mathrm{~m} / \mathrm{s}$ (in the middle of the graph) increasing as the Doppler effect is velocity sensitive. At some point the atoms move so fast that they will not be able to scatter enough photons to be effectively cooled and trapped. Around $30 \mathrm{~m} / \mathrm{s}$ the force due to the Doppler effect goes to zero 60 .

shown in Figure 8.

In Figure 8 the line passes through the origin showing zero velocity. We can see the force has the linear slope $\cong-\beta v$, the slope itself defining a damping coefficient. The radiation pressure in this situation has a net damping effect on atoms that are within that linear region [61]. Random direction of atoms gives us the net diffusion as,

$$
\text { Diffusion: } D \cong 2 \frac{\left(\Delta_{\text {step }}\right)^{2}}{\Delta_{\text {step }}} \cong 4 \Gamma(\hbar k)^{2}
$$

Radiation force lowers the energy and diffusion raises the energy and their net effect is given as,

$$
\delta=-\frac{\Gamma}{2}, S_{o} \ll 1, k_{B} T=\frac{D}{\beta}=\frac{\hbar \Gamma}{2}
$$




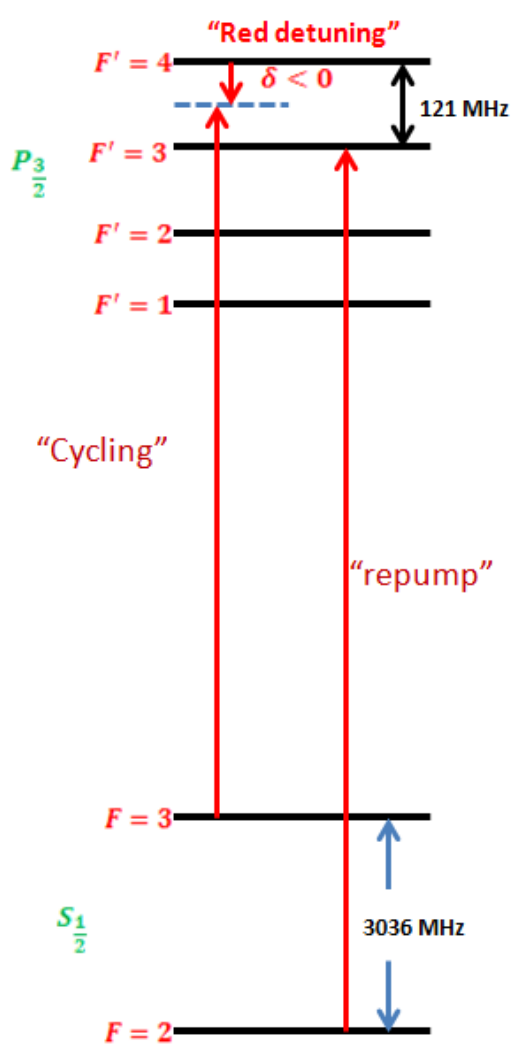

(a)

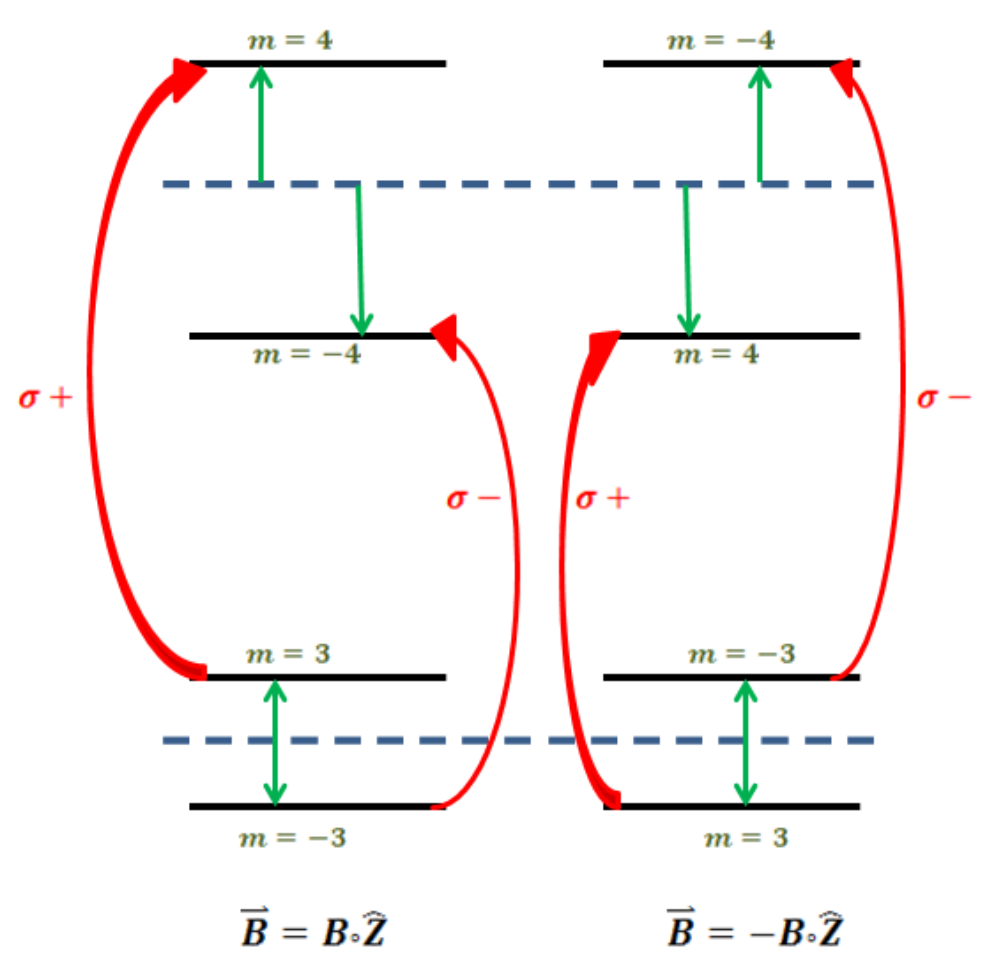

(b)

Figure 9: (a) Rubidium ${ }^{85} \mathrm{Rb}$ level structure (b) The shift of the energy level of atoms due to the presence of magnetic field (Zeeman Effect for ${ }^{85} \mathrm{Rb}$ atom) (Figure regenerated from $[62])$.

This temperature limit is known as Doppler limit and capture velocity for laser beam of diameter $d$

$$
\frac{1}{2} v_{c}^{2}=\left(\frac{E}{m}\right) d
$$

\subsubsection{Forming a trap}

To understand how a MOT is a trap rather than performing only cooling, we take the example of Rb atom as it's also the subject of this $\mathrm{PhD}$ experiment.

Figure 9 (a) shows the level structure for ${ }^{85} R b$ isotopes. In ground state there are two different hyperfine levels that correspond to different spins of atoms, e.g. $F=2$ and $F=3$. These two hyperfine structures are split by $3036 \mathrm{MHz}$ in frequency. Excited levels corresponding to $P_{\frac{3}{2}}$ states are reach by a laser of wavelength $780 \mathrm{~nm}$. Four different 
values are possible for hyperfine spin $F^{\prime}=1, F^{\prime}=2, F^{\prime}=3$ and $F^{\prime}=4$ respectively. Levels of interest for us are $F^{\prime}=3$ and $F^{\prime}=4$ that are split by a hyperfine splitting of 121 MHz. Most of the laser using Doppler cooling and trapping is at the resonance frequency which is close to that of $F=3$ driven to $F^{\prime}=4$. This transition is called the "cycling transition" as shown in Figure 9. When atom goes from $F=3$ to $F^{\prime}=4$ state then it has to decay back down to $F=3$ level, because emitted photon will only carry 1 unit of angular momentum. We used laser light that is little bit below the atomic resonance frequency called "red-detuning" where $\delta<0$ (detuning is negative), in this situation we have cooling. In case an atom misbehaves and absorbs photon at $F^{\prime}=3$ level, there is a chance that it can escape this cycling transition and fall down to $F=2$ level. At this point it will no longer be started by this light cycling transition. To rescue these atoms and to stay in this cycling transition, we shine a light on another transition frequency, driving $F=2$ to $F^{\prime}=3$ level. This light is often referred to as "re-pumping". We generate this light on optical table by frequency modulation of the cycling light using Acoustic Optic Modulator (AOM). By using the combination of these two laser frequencies we can indeed create our magneto optical trap (MOT) 62.

Figure 9(b) shows how the MOT works by the shift of energy level of the atom due to the presence of magnetic fields. Let's consider the situation $\left(\vec{B}=B_{o} \hat{Z}\right)$, where the magnetic field points up, that is; it's point up in +z-direction, the different energy levels also have different projections of their angular momentum along the + z-axis. For the state that has a projection of $m=3$ along the +z-axis, in the ground states, its energy goes up by a certain amount in the magnetic field. For the excited state with the largest value $(m=4)$ the increase in energy is more than that of energy level in ground state. The opposite is true for the states that have negative values (i.e. in case of opposite velocity the sign should be negative but not the magnetic field $\left.\left(\vec{B}=-B_{o} \hat{Z}\right)\right)$ of the projection of their angular momentum along z-axis, $m=-3$ level drops down, $m=-4$ level in excited state drops down even more. To see the effect on transition frequency for this atom, we consider the transition that is driven by the light that has sigma plus $\left(\sigma^{+}\right)$polarization, that will 

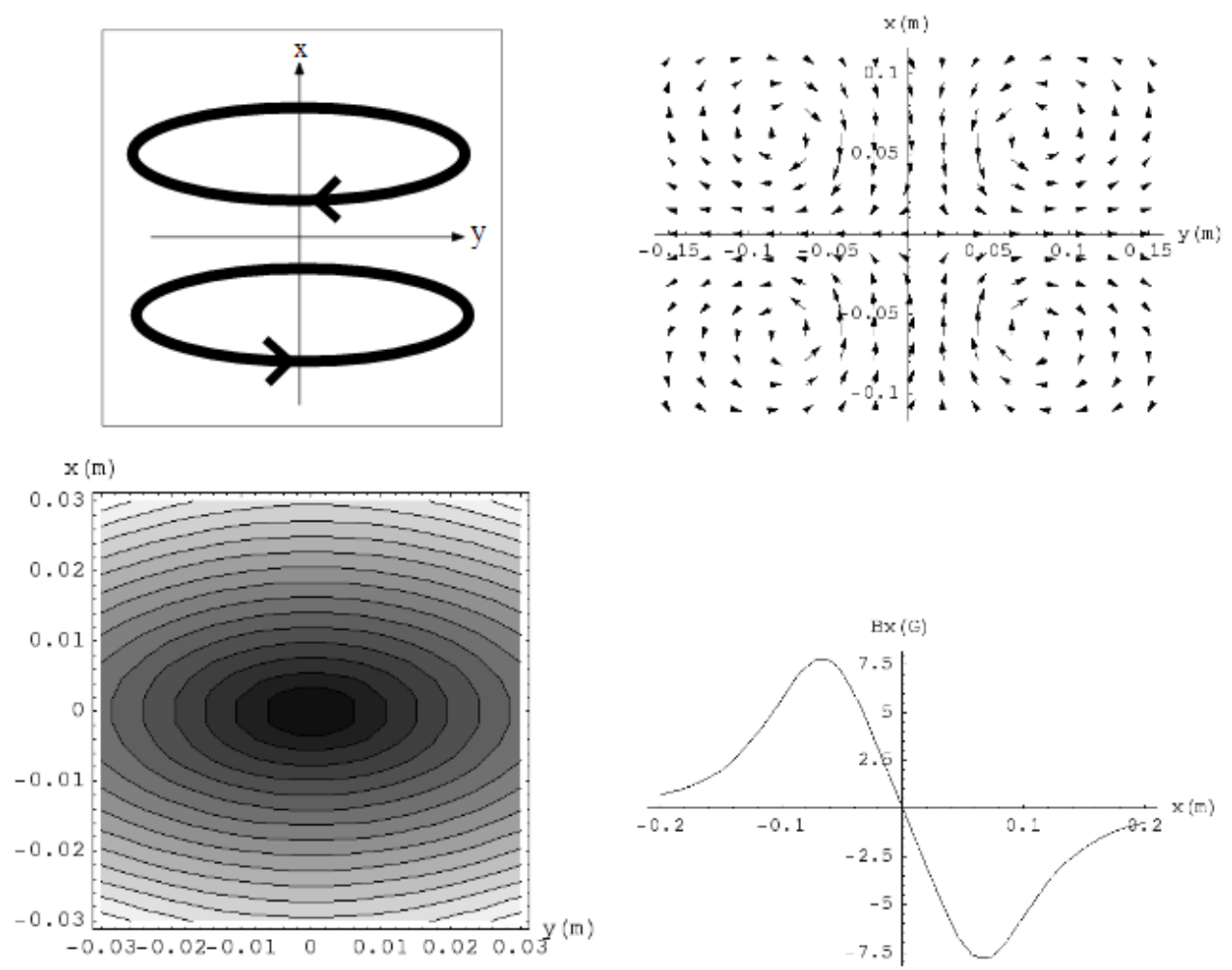

Figure 10: Magnetic field generated by anti-Helmholtz coils, Upper Left: Current direction of the coils and their orientation, Upper Right: Quadrupole centered at origin with a plane vector field, Lower Left: Plot for the central quadrupole region, Lower Right: z-component of the magnetic field on x-axis, also we can see the linearity of the field near the origin [65]. Note here we showing circular coils but for our experiment we are going to used rectangular coils. We want to show the difference between circular and rectangular coils.

increase the angular momentum of the atom along z-axis [62] [63]. $\sigma^{+}$transition will have atomic resonance frequency that is increased in the presence of magnetic field $\left(\vec{B}=B_{o} \hat{Z}\right)$. On the other hand, $\sigma^{-}$transition will have resonance frequency which diminishes in the presence of $\left(\vec{B}=B_{o} \hat{Z}\right)$ magnetic field, if we flip the orientation of magnetic field (i.e. $\vec{B}=-B_{o} \hat{Z}$ ) that points downs but we still fix the definition of our $m=3, m=4$ and $m=-4$ to z-axis [64.

Figure 10 shows the graph of magnetic field created by a pair of Anti-Helmholz coils against z-axis. We can clearly see that at the origin of the graph where the atoms are trapped, the magnetic field is zero. When we send $\sigma+$ light that is coming from below and $\sigma$ - light that is coming from above then atoms are likely to scatter photons upwards 


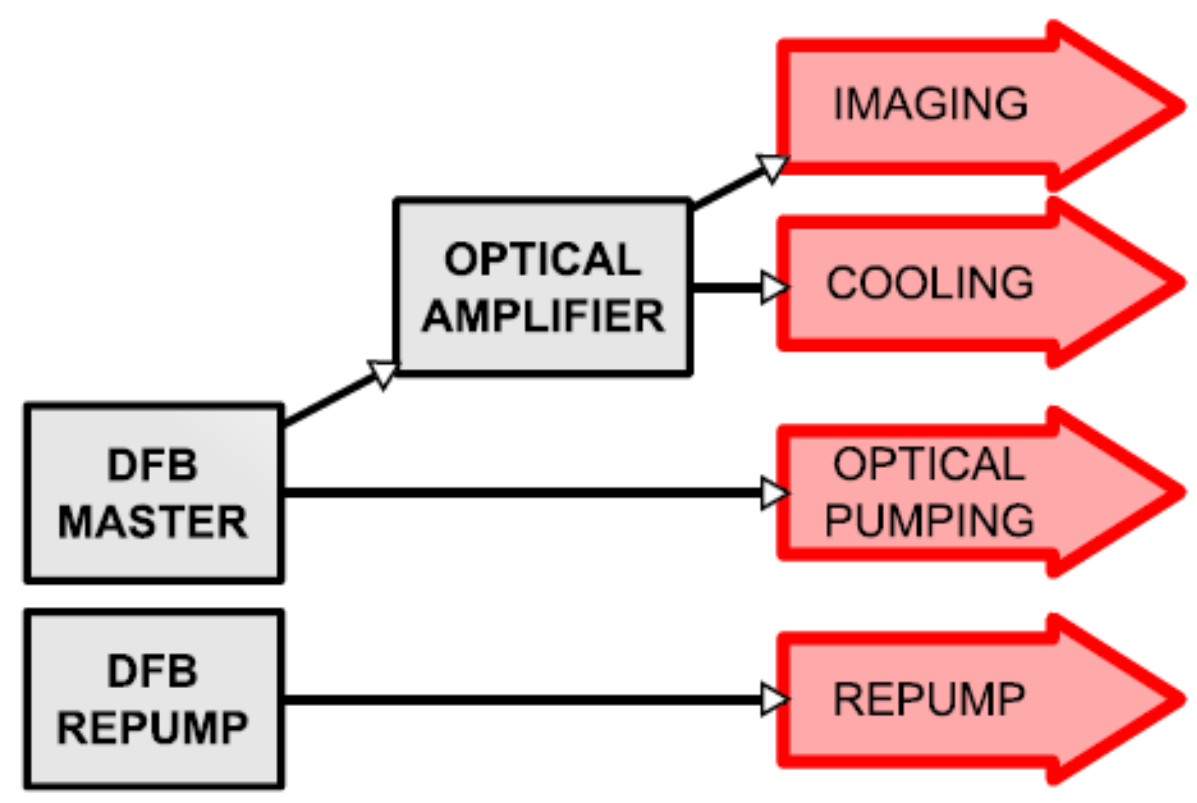

Figure 11: DFB laser scheme 66.

from the origin which pushes the atoms back towards origin and likewise when they go to the region space where the B-field points down they are likely to scatter the light to push them back and that gives us a restoring force on the atom and allows to trap us [60].

\subsection{MOT set-up}

We introduce a typical MOT experimental set up to understand the basics in more details. We used Distributed Feedback (DFB) laser diode for this experiment, which is also the subject of our experiment. The DFB laser diode is a perfect match with the grating structure incorporated with the Atom Chip. Further details about the DFB laser diode is discussed in [66. Only draw back of DFB laser diode is the small coarse, the linewidth of few $\mathrm{MHz}$ and tuning range of some $\mathrm{nm}$.

Figure 11 shows the two DFB laser system in which diode and electronics are manufactured by TOPTICA company. Each laser provide $120 \mathrm{~mW}$ of power. Figure 11 also shows the DFB master laser and DFB repumper laser. DFB master laser generates light with a frequency close to $F=3 \longrightarrow F^{\prime}=3$ transition. More details of DFB master and DFB repumper lasers can be found [66]. 


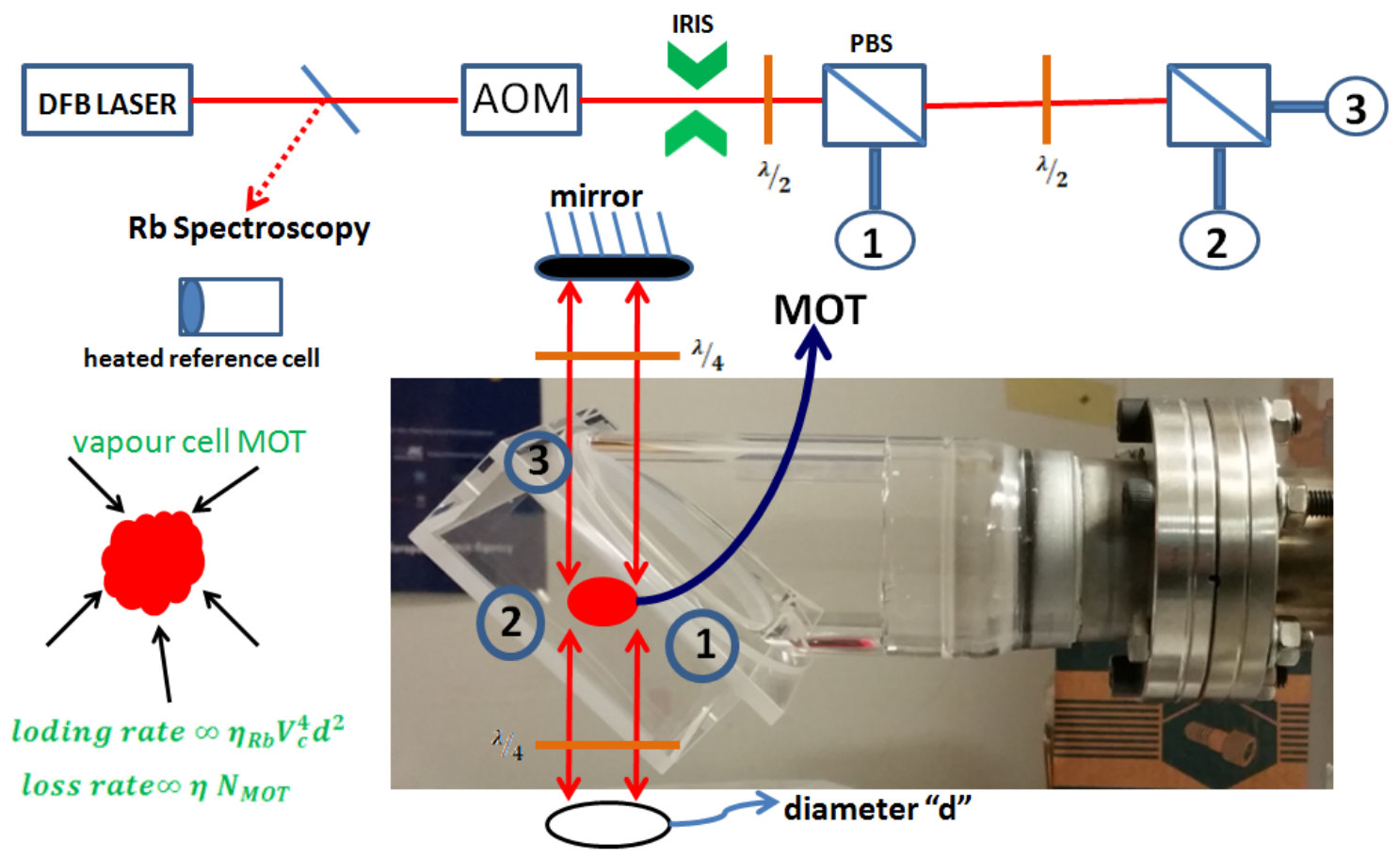

Figure 12: Brief schematic of Rb atom set-up

Figure 12 shows the DFB laser that, let's say, generates 70 to $120 \mathrm{~mW}$ that is near to the resonance transition frequency, and then small portion of light splits into the area called Rb spectroscopy. When this small portion of light goes into this Rb spectroscopy that contains a heated reference cell and has a $\mathrm{Rb}$ vapor just above room temperature which makes this light able to tell us where the laser is with respect to Rb. Other main portion of light continues to rest of the apparatus, all of it first passes through an AOM (Acoustic-Optical Modulator). AOM work as a coupling between the photons (that comes from the laser beam) that passes to the acoustic-optic crystal and phonons of the crystal excited by the RF input signal, we received an output of zero order and several diffraction components. Optical frequencies differ by multiples of the induced acoustic frequency. For our experiment we used negative/positive first order but sometimes if required power is very low then we can used second and third order. Figure 13 shows different orders with respect to their frequencies, more details can be found in [66]. Figure 13 shows the scheme that allow us to lock the laser to the peak of $\left(F=2 \longrightarrow F^{\prime}, F=2: \longrightarrow F^{\prime}=3\right)$ crossover saturation line (CO1). We can also see in Figure 13 that by the addition of AOM we have 


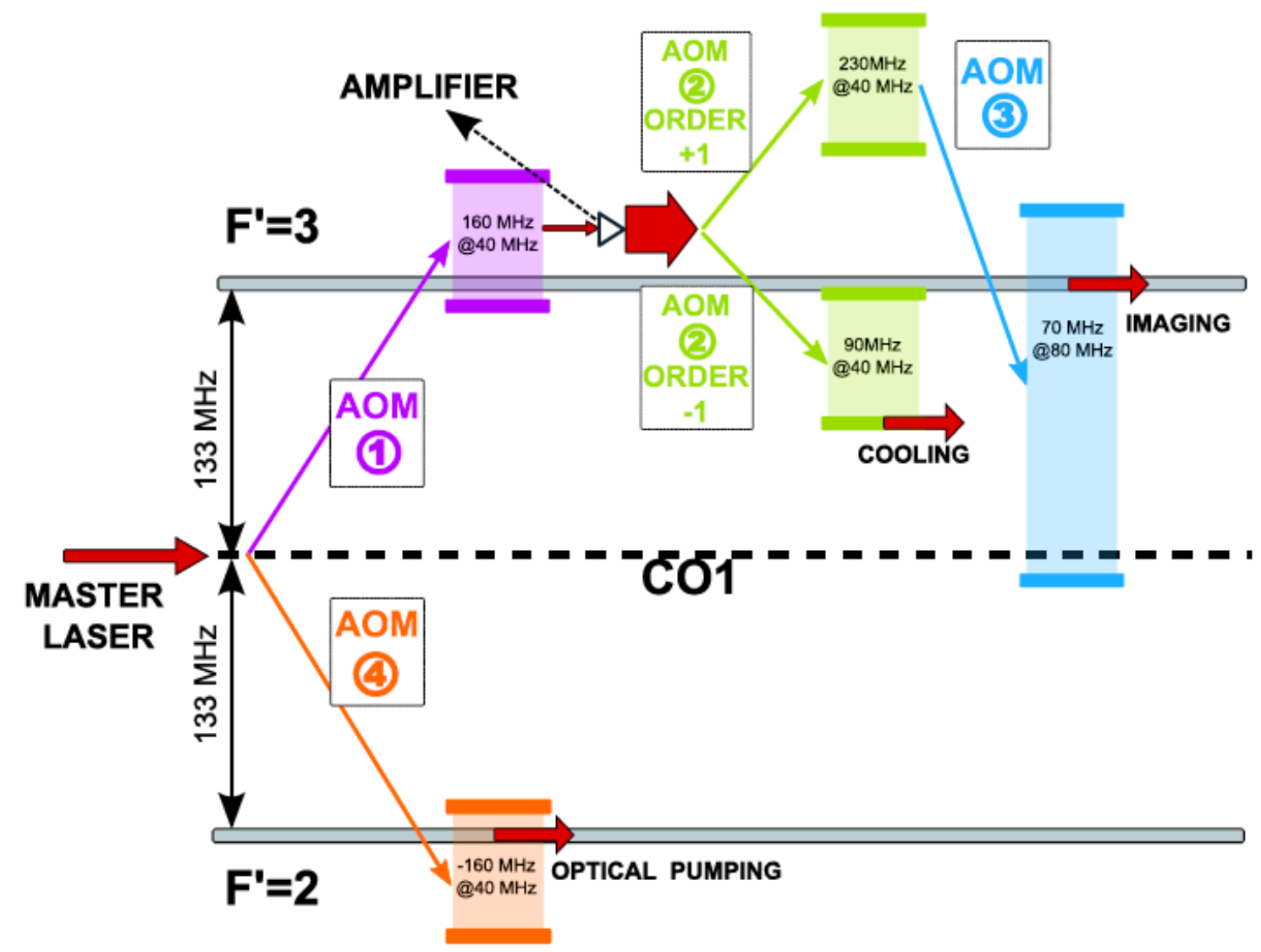

Figure 13: Master Laser (DFB) generated the frequency, these frequencies are related to crossover CO1, figure also showing the bandwidth and center frequency of each $\mathrm{AOM}$ 66.

the cooling light and red detuned by several natural line-widths from $F=2 \longrightarrow F^{\prime}=3$ cooling transition.

After AOM we have two options, there is one part going through a mechanical shutter to completely block the beam or we can have, if necessary, a reliable iris to do beam shaping. Further, one usually has an iris with very small opening after the AOM to let only the desired diffraction order pass. After passing through IRIS, it goes to the portion of experiment where the laser power splits between different optical beams and this is how we do it; we start with linear polarized light and send it through so called $\frac{\lambda}{2}$ plate (also known as half retarder). $\frac{\lambda}{2}$ plate takes linear polarization light and rotates it along the axis of propagation. This rotated light then light beam passes through PBS (Polarizing Beam Splitter), which takes one polarization of light and sends it along one path and different linear polarization light to another path. By rotating the $\frac{\lambda}{2}$ plate we can vary 
the ratio between reflected (e.g. in our case 1 as shown in Figure 12) and transmitted light. We repeat this scheme as shown in Figure 12, so that we split the light further into three beams e.g. beam 1, beam 2 and beam 3, which will be sent on the atoms along three cardinal directions. Simplified scheme of our vacuum chamber is shown in Figure 12 which shows a glass cell with big windows where all beams pass through. Here it is worth mention that for the simplicity we didn't introduce the details of our Atom Chip and the easy replacement of the Atom Chip. In later chapters we will discuss the detail of the Atom Chip and it's easy replacement.

This UHV (Ultra High Vacuum) glass cell is fitted to the vacuum pump. Inside this cell $\mathrm{Rb}$ atoms are introduced at roughly room temperature and are to be initially trapped and cooled by a MOT. Beam 1, 2 and 3 are arriving and they shine on the atoms from different windows of UHV glass cell. For simplicity we have shown only one access of beam (beam 3 ) in Figure 12. Beam 3 is along z-axis and it passes through $\frac{\lambda}{4}$ plate which retards the light into circular polarize atom as required for a MOT. This light then goes through the chamber passing through the Rb atoms, comes back out of the chamber and then passes

$\frac{\lambda}{4}$ plate to reflect back by mirror and passes through again from the same $\frac{\lambda}{4}$ plate that reverses the circular polarization of light. All this mechanism is shown in Figure 12. All three beams have a particular diameter $(d)$, also shown in Figure 12 . Diameter $(d)$ of beam is important because it give us the understanding of the loading rate of atoms in our MOT. Let's consider that we are dealing with the specific vapor cell MOT that pulls $\mathrm{Rb}$ atoms out of a high temperature vapor and pulls them into a trap where they will be cooled. Even though the high vacuum condition, there is still a dilute gas surrounding these atoms. The loading rate of atoms that are loaded into MOT is proportional to $d^{2}$ (area cross section where vapor has to hit in order to fall into MOT), and to capture velocity.

$$
\text { loading rate } \alpha \eta_{R b} V_{c}^{4} d^{2}
$$

Where, $\eta_{R b}=$ Density of Rubidium $(\mathrm{Rb})$ in the chamber 
$d^{2}=$ Area of the MOT (e.g. that should be a cross section that vapor has to hit in order to fall into MOT)

$V_{c}^{4}=$ Fraction of atom in room temperature that has a velocity that lower then the capture velocity (i.e. If velocity is high then it fly through the MOT if lower then it capture in MOT).

We can measure this loading rate by extinguishing the MOT and then suddenly turning them back on and seeing how quickly our fluorescence signals rise. Loading rate is balanced by some loss rate,

$$
\text { loss rate } \alpha \eta N_{M O T}
$$

Where,

$\eta=$ Density of the particles in vacuum chamber

$N_{M O T}=$ Number of atoms that are stuck in background vapor

Loss rate is a common situation that the loss is dominated by collision, where the room temperature background gas knocks atoms out of the MOT. We have loss rate which is proportional to the number of atoms that are stuck by this background vapor. Under the special condition, the pressure inside UHV chamber is completely dominated by the $\mathrm{Rb}$ vapor pressure [56 [67.

\subsection{Difference between traditional and Mirror MOT}

Atoms can be trapped and cooled through different techniques such as guided injections from a cold atomic beam coming from low-velocity intense source called LVIS [68 [69 [70]. We used MOT in our experiment because of its versatility and reliability. We already discussed about MOT in the previous section. In this section we will discuss special type of MOT with different configuration. The purpose is to better match the requirements imposed by our experiment.

Now if we consider the geometry of a typical 6-beams MOT, then we can easily derive 
that the distance available between the MOT and the Atom Chip (Atom Chip is discussed in next Part of this thesis in detail) shouldn't be shorter than the linear dimensions of the Chip itself. For this purpose one or more laser beam should be blocked. Thus for our experiment we used four counter propagating beams instead of six. Our Chip with holder has a dimensions of $20 \times 25 \mathrm{~mm}$ (discussed in Chapter 5). The diameter of the MOT beams to determine how close we can get with the chip is $35 \mathrm{~mm}$ as mentioned in literature [66] and for the $34 \mathrm{~mm}$ as mentioned in in literature [71]. This transfer has to be realized without heating or loosing the atom [66].

The concept of a Mirror-MOT comes from the presence of a surface (which is coated with gold) of Chip. In our experiment we introduced the atom chip which cuts the direction of upper and lower beams and finally permits to have a complete MOT configuration a few millimeter (mm) away from the surface of Chip. When the surface of the Chip is fabricated by gold $(\mathrm{Au})$ then it works as a very good mirror for our lasers at $780 \mathrm{~nm}$.

There are some alternative techniques as well for trapping atoms that come very close to the surface, the gravito-optical surface trap (GOST). GOST principle is the combined effect of repulsive force and gravity on atoms by the evanescent wave of blue detuned laser beam impinging on the back face of a horizontal dielectric surface [72]. In this way a planar matter waveguide is realized and used to study quasi-2D atoms [73] [74]. This technique is not suitable for our experiment because in this technique the trap needs a complete optical access to the back of the optically homogeneous surface using the mirrorMOT approach. In the end the best technique that suits our experiment is to bring the MOT very close to the Chip surface. The basic difference between standard MOT and mirror-MOT is shown in Figure 14.

\subsubsection{Working of Mirror-MOT}

The first basic concept of mirror-MOT has been used in 1996 to realize a very unique version of a standard MOT, the so-called Pyramidal MOT. The idea behind this is that a circularly polarized laser beam changes helicity (combination of the spin and the linear 


\section{Traditional MOT}

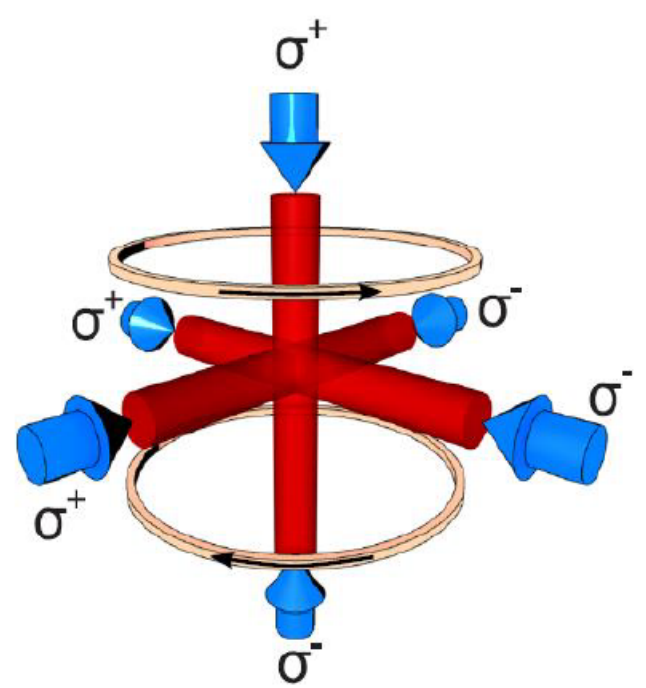

The laser beam and the coil geometry for usual 6-beam MOT with a quadruple configuration

\section{Mirror MOT}

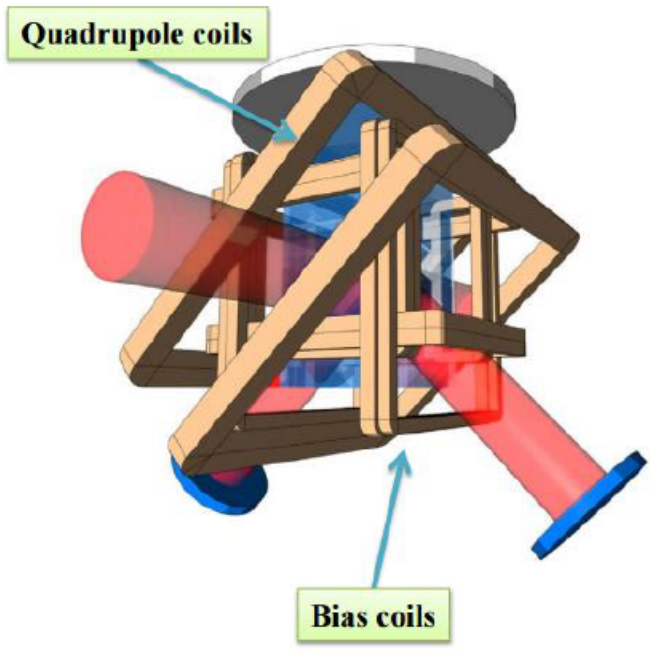

Laser beams striking on chip inside Vacuum chamber, with Bias and Quadruple coils

Figure 14: On left: Traditional MOT with six counter propagating beams, On Right: MOT near Atom chip with four counter propagating beams 66

motion of a subatomic particle) upon reflection on a mirror [75].

Figure 15 shows the Pyramidal MOT, in which a single laser beam is incident on a pyramidal hollow mirror. When the beam helicity is incident on the hollow region of mirror it gives us the following result: along the incident beam direction the helicity of the forward beam is the same as that of the incident beam. The reason behind this is that it has been reflected twice by the $45^{\circ}$ mirror faces. Moreover, the perpendicular plane helicity is opposite to that of the incident beam along any direction, it's because the beam has been reflected only once 72$]$.

One can easily understand that this is exactly the beam configuration of a typical 6-beams MOT, it just needs a quadrupole field generated by a pair of coils with axis parallel to the beam [72]. Note here, in Figure 15 the coils are in rectangular shape but in actual literature circular coils are used. In literature [76] conical shaped hollow mirrors were used and tested [77] [76]. Pyramidal MOT give us further insight into the planar mirror-MOT configuration by adopting partially reflection mechanism, which is shown in Figure 16 . Figure 16 showing the 4-beams MOT configuration replicated by 6-beams MOT. Two 


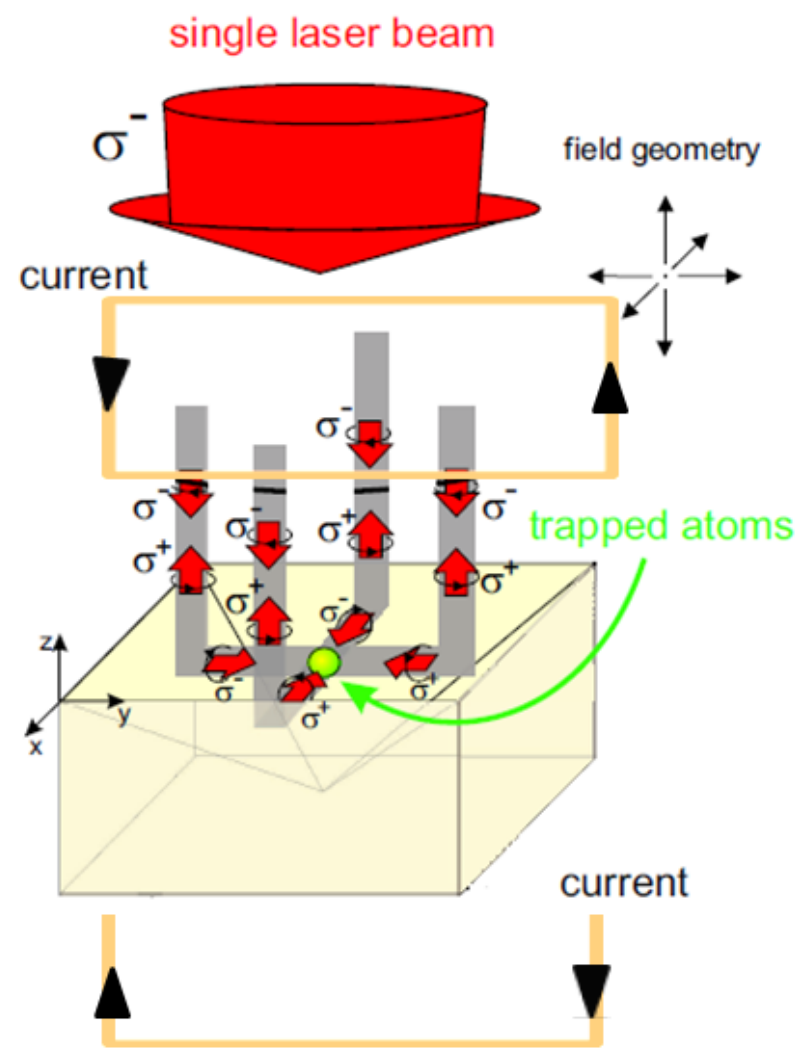

Figure 15: Set-up for Pyramidal MOT, circularly polarized laser beam showing as red arrows and black small arrows indicated as helicities, polarization indicated as $\sigma^{+}$and $\sigma^{-}$. Trap atoms come at the center (Figure regenerated from 72$]$ ).

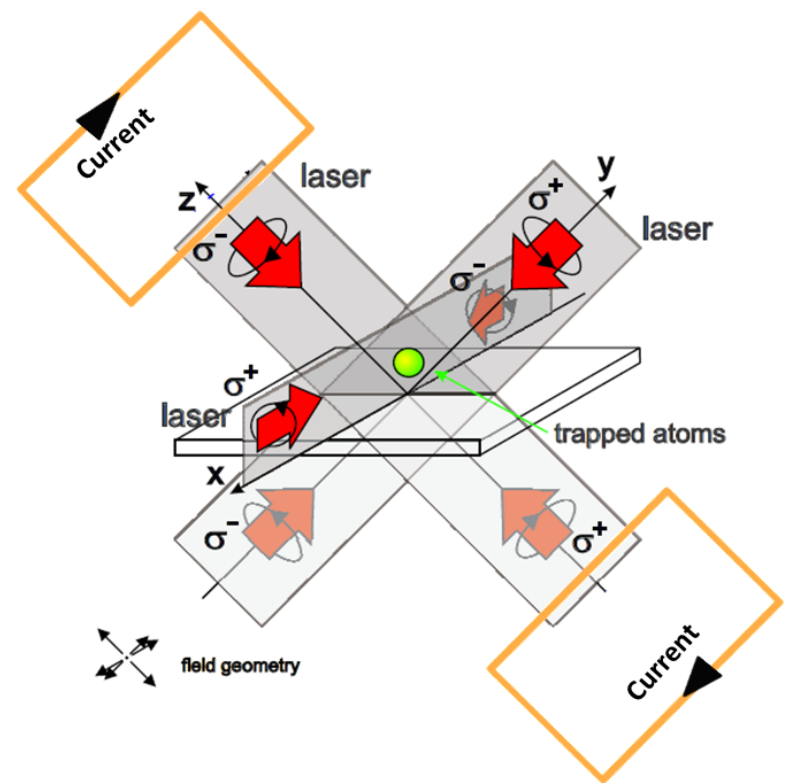

Figure 16: Schematic representation of mirror-MOT beams and field configuration in which surface should be fully reflected for circularly polarized laser beams (Figure regenerated from 72 ). 
beams are impinged on the mirror at $45^{\circ}$ from opposite directions. One beam is parallel to the mirror as shown in Figure 16. The two beams lay in the plane perpendicular to the beam that is parallel to mirror. Figure 16 shows the rectangular coils but in actual literature these are circular. For Figure 16 the quadrupole coils axis will be oriented along the direction of the beam of different helicity, and that should be tilted at $45^{\circ}$ with respect to the mirror plane. At the end, the center of quadrupole magnetic field should be situated at the beam overlapping region. In this way we can achieve the usual MOT geometry that is realized in the half space defined by the mirror. This technique was first tested in 1999 by the group of T. Haensch [78] and later by J. Schmiedmayer [79]. In the next Chapter we will discuss mirror-MOT in more detail and we will show that how our experiment fits the mirror-MOT configuration. 


\section{Experimental Set-up for Cold Atoms}

\subsection{Introduction}

In this chapter we will explain the details of the experimental setup. This includes the different components of the vacuum system, the required optics and the magnetic system. Due to its particular complexity the Atom Chip itself will be treated in Chapters 4 and 5. The vacuum system can be divided into several section: The central part consists of a rectangular cell made entirely out of glass. Other parts made of steel include the required pumping system, the Rb vapor source and a specially designed load-lock system for the rapid exchange of the Atom Chip. All those components will also be described in the following sections.

\subsection{Vacuum System}

For cold atom experiments, the heart of the experiment is the Ultra High Vacuum (UHV) chamber, where all the laser beams interact, trap the atom and in our case also atom chip and $\mathrm{Rb}$ atoms are present. The main operation takes place inside the chamber. Our aim was to design a chamber that is compact, not very costly, allows for easy optical access, permits our atom chip to be easily exchangeable, requires minimum space for BIAS and anti-Helmholtz coils, and provides good access for vacuum pump.

At the beginning, we want to introduce a company manufactured (MDC) steel vacuum chamber MCF600 series [80]. This steel vacuum chamber has 6 Multi-CF Spherical Octagon ten-port Multi-CF Fitting, consisting of a hollow sphere intersected by two $6 \mathrm{CF}$ sealing surfaces and eight $2 \frac{3}{4} \mathrm{CF}$ sealing surfaces 80 . The main problem we faced for this steel vacuum chamber is the compatibility; this UHV chamber was rather large and our BIAS and $45^{\circ}$ anti Helmholtz coils would have required a huge amount of current to be run through. Water cooling would have become a necessity. Another issue for this chamber were the $45^{\circ}$ MOT beams, it's difficult to have sufficient optical access for these beams 


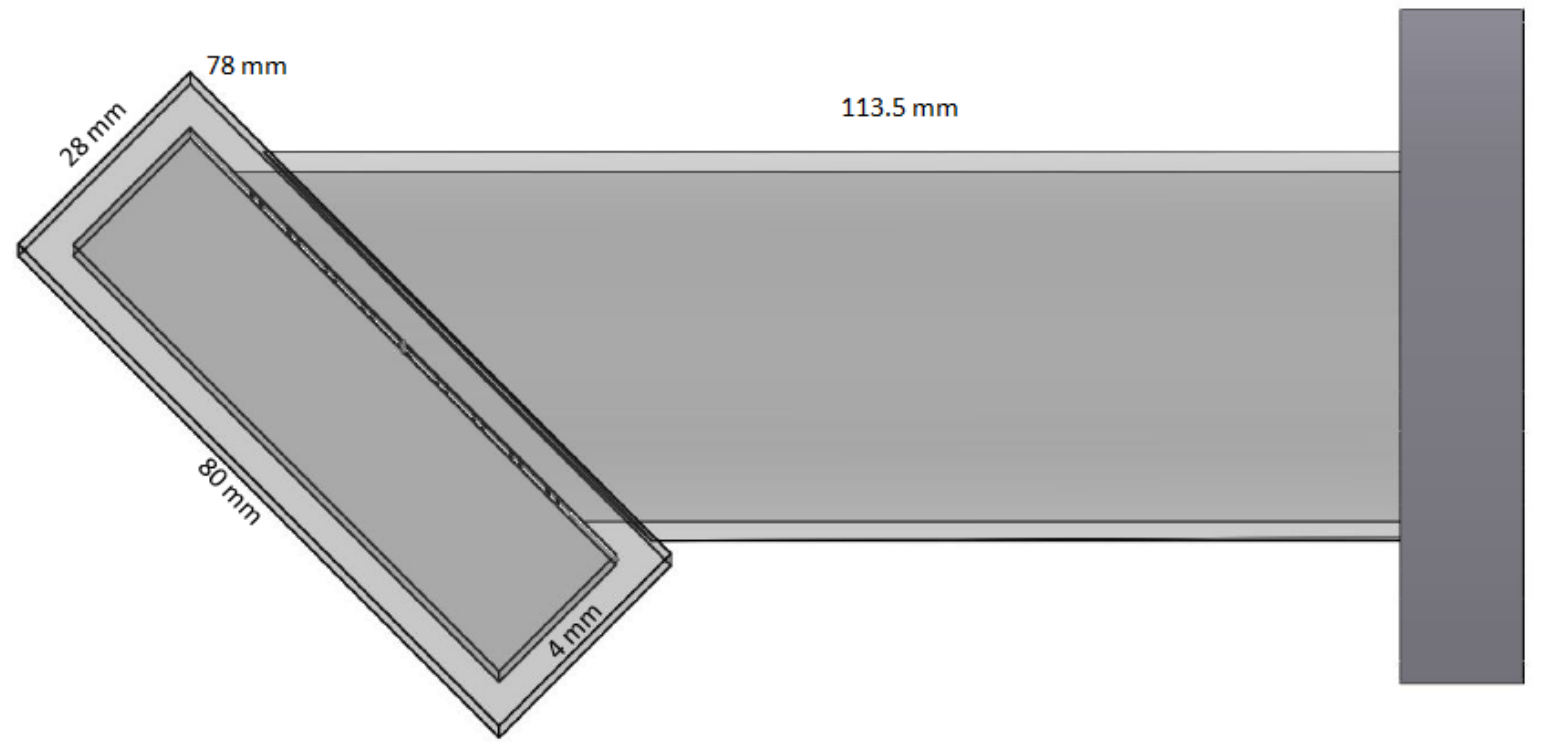

Figure 17: Glass cell which we used in our Atom Chip experiment (drawing of the cell with all dimensions is further mentioned in Figure 59 of Appendix A).

in the presence of the additional beams required for operating the photonic structures on the Atom Chip (cf. Chapter 5).

After facing the above mentioned difficulties we decided to design a custom UHV chamber and then ask a company to manufacture it. For the optical access we decided to go with a glass cell rather than steel chamber. After all calculation and simulations we managed to design a UHV glass cell that really fulfills our demands for the experiment. the projected UHV glass cell is shown in Figure 17 .

We can see that this design ensures ease of optical access. The glass tube is $113.5 \mathrm{~mm}$ long and the base is connected to the glass tube under $45^{\circ}$ (with respect to base). The base of cell is $80 \mathrm{~mm}$ long and $28 \mathrm{~mm}$ in height. At the end of tube we have CF-40 flange which has an inner free diameter of $38 \mathrm{~mm}$. This UHV glass cell is also ideal for BIAS and anti Helmholtz coils. We only need a minimum current to run in the coils. An extra water cooling these coils is not needed. After designing the glass cell we look towards the manufacture companies who can produce such type of UHV glass cell. In the beginning it seemed difficult to find any company who could build this type of glass cell, the main reason being the crucially short distance between the base and tube but in the end Hellma 


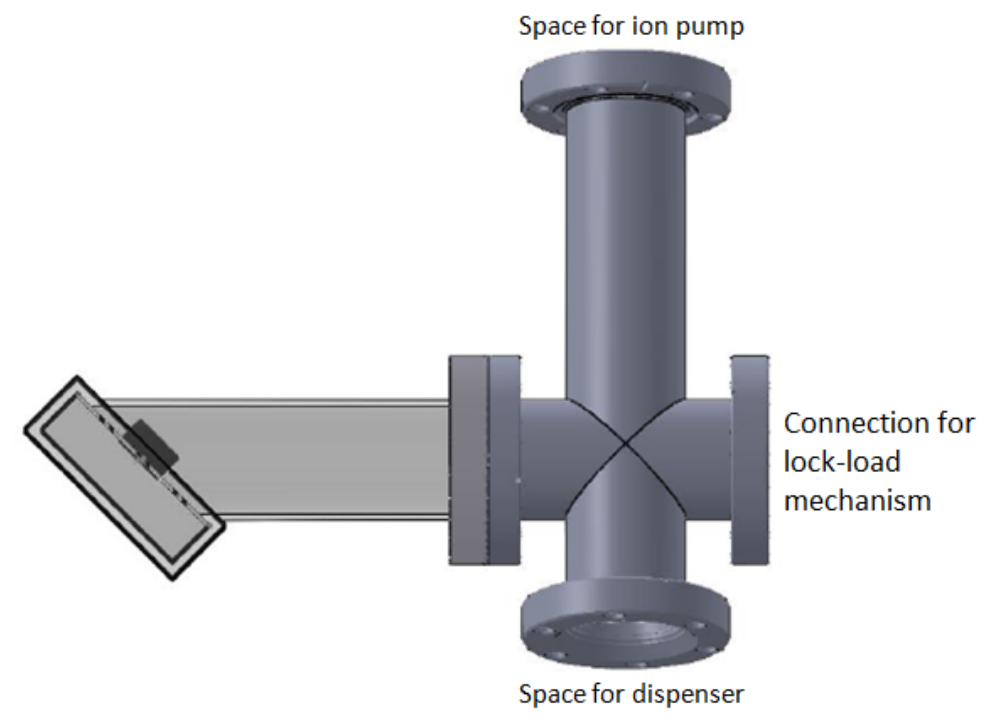

Figure 18: Glass cell with four cross pipe. Drawing of the pipe is shown in Figure 60 Appendix A.

agreed to manufacture our UHV glass cell.

\subsection{UHV Glass cell with different vacuum components}

Next we have to look for other parts that are going to be attached to the UHV glass cell. We were looking for some kind of metal pipe that will connect other vacuum components with glass cell. We wanted the pipe length to be as small as possible because we needed to minimize the distance the chip needs to travel from our load-lock system to the glass cell. We want our set-up compact, and we designed a metal pipe to suit our needs. This "four way cross pipe" has four openings and each opening has a CF-40 Flanges that is further connected with UHV glass cell, vacuum pump, Rb dispenser and gate valve. The four cross metal pipe attached to the UHV glass cell, space for Rb dispenser and space for vacuum pump is shown in Figure 18.

Now we were left with last opening of CF-40 Flange pipe where a gate valve was going to be attached. The gate valve plays an important role because it will separate the UHV of the chamber from the surrounding atmospheric pressure. We placed the gate valve because, when our experiment is running, then the gate valve will be open and when our 


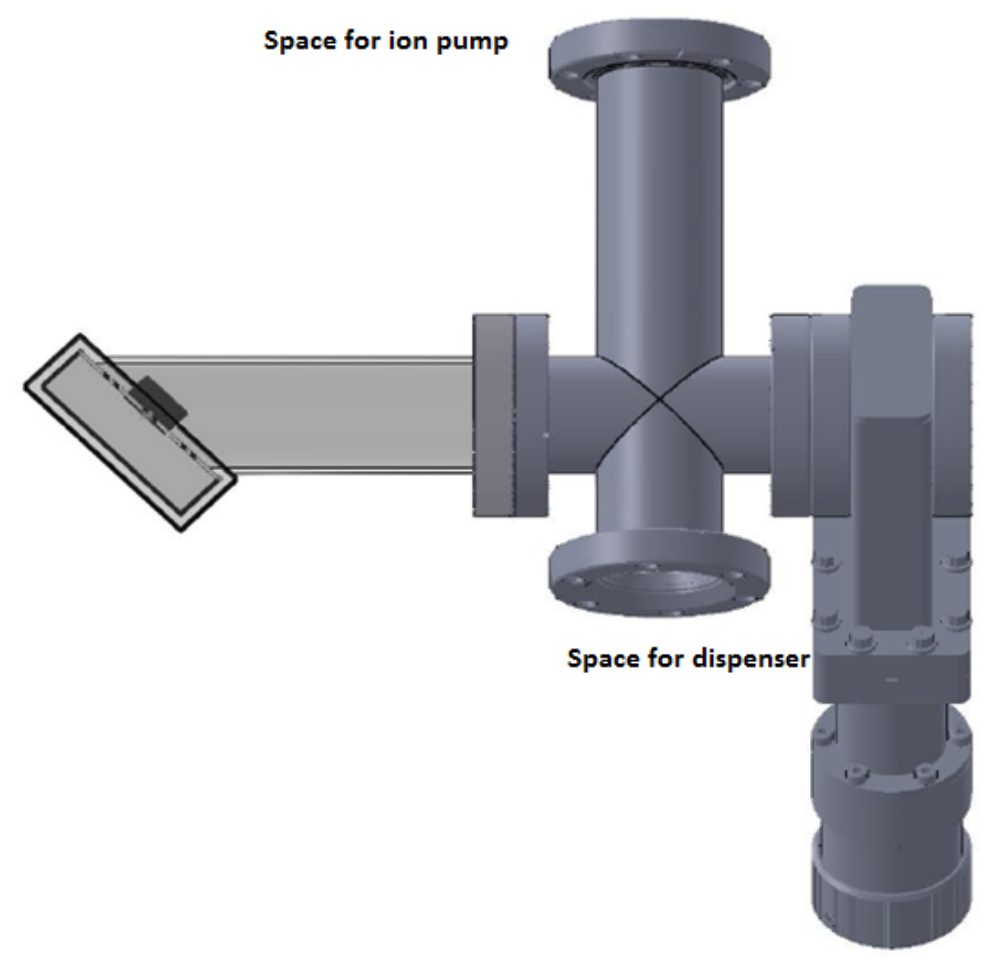

Figure 19: Glass Cell attached with four cross pipe and gate valve, Atom Chip showing in the center of cell.

experiment is not running or when we have to replace an atom chip, then the gate valve will close to maintain the vacuum inside the glass cell. Glass cell, four cross pipe, space for $\mathrm{Rb}$ dispenser, space for vacuum pump and gate valve is shown in Figure 19 .

For atom chip we introduced a $455 \mathrm{~mm}$ long rod. Atom chip will be attached at one end of the rod and on the other end a CF-40 Flange will be attached. Further demonstration of the rod is shown in Figure 20.

We can see the holes in the metal rod, these $2 \mathrm{~cm}$ holes are for ensuring good vacuum. When the experiment is in progress and the rod brings the atom chip in the glass cell then vacuum pump is in progress simultaneously and these holes in rod will provide more access to vacuum pump to maintain the high pumping speed.

At the end we definitely need something that moves the rod back and forth frequently. So we decided to go for a linear shift mechanism. This linear shift mechanism will move the rod back and forth and provide proper sealing of the rod against atmospheric pressure for maintaining the UHV condition of the vacuum system. The length of this linear shift is 


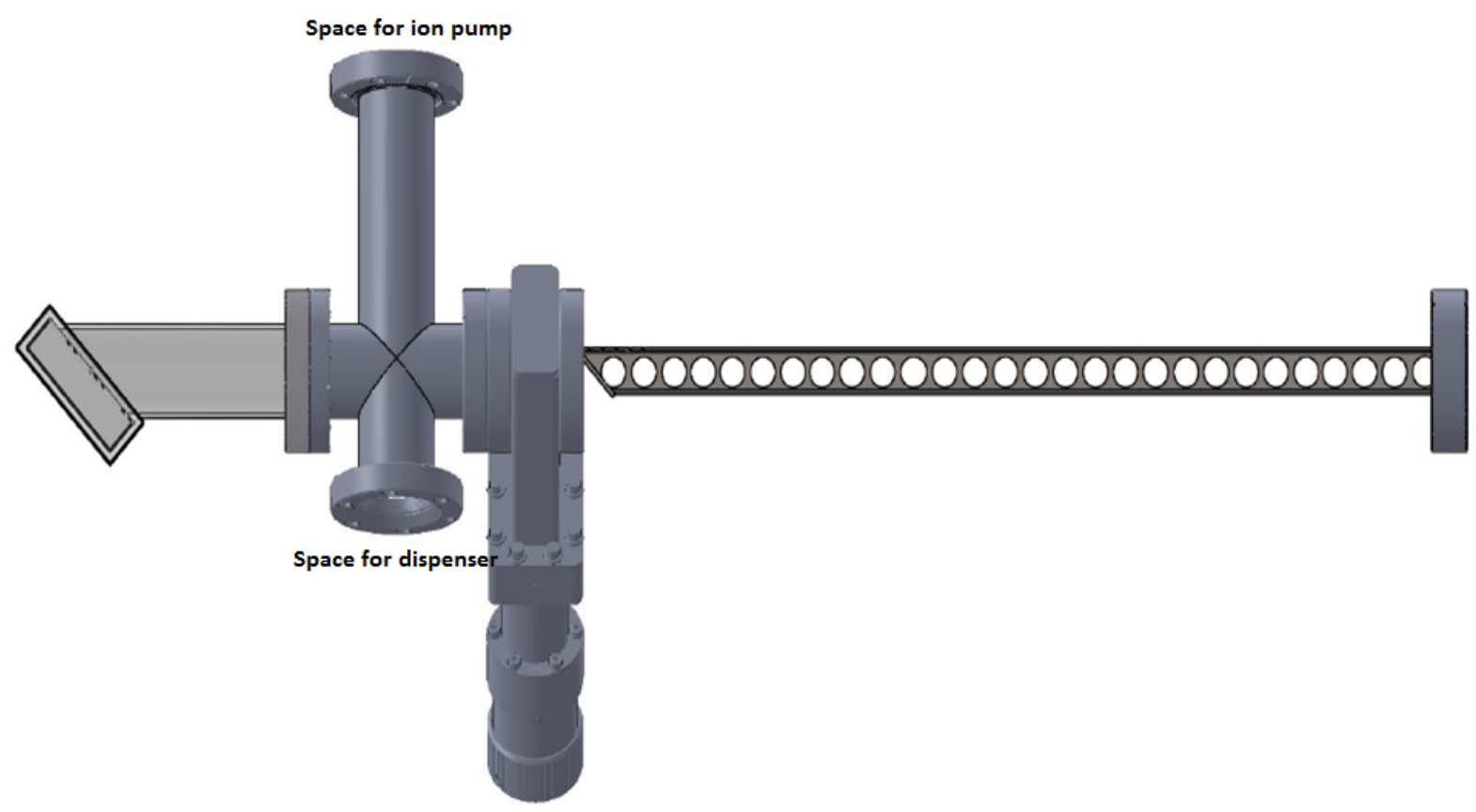

Figure 20: Glass Cell attached with four cross pipe, gate valve and metal rod that will bring the Atom Chip at center of glass cell. Metal rod drawing is shown in Figure 61 Appendix A.

$455 \mathrm{~mm}$ (equal to rod length). Linear shift mechanism and rod attached through CF-40 Flange is shown in Figure 21.

\subsection{Optics for the Experimental Set-up}

Laser system plays an important role in Magneto Optical trap (MOT) experiment. We need correct frequencies and alignment of laser light to trap the atoms. For MOT we require light at two frequencies; cooling and re-pumping. These two frequencies with the frequency of MOT are illustrated in Figure 22, along with the hyperfine structure of the ground $5 s^{2} S_{\frac{1}{2}}$ and excited $5 p^{2} S_{\frac{3}{2}}$ states of the $D 2$ transition in ${ }^{87} \mathrm{Rb}$. Here we have to remember that the hyperfine structure described in section 2.2 .1 is used for ${ }^{85} \mathrm{Rb}$ for comparison but our experiment is based on ${ }^{87} \mathrm{Rb}$ atoms.

Figure 22 shows the hyperfine structure for ${ }^{87} \mathrm{Rb}$. We can see that for cooling light must be red detuned by a few linewidths from resonance with respect to the $F=2 \rightarrow F^{\prime}=3$ transition. To collect a large number of atoms, one would like as much light as possible 


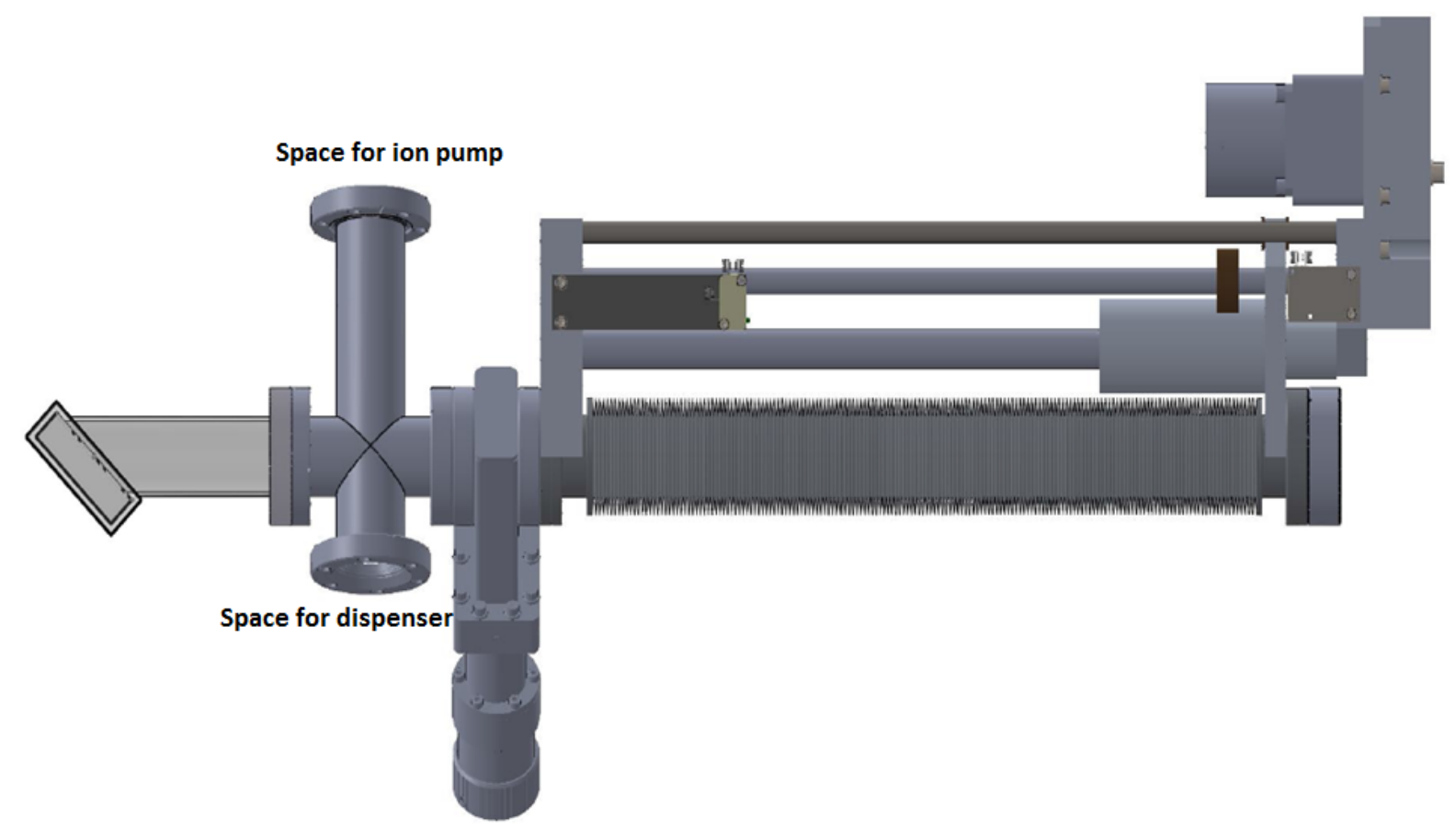

Figure 21: Linear Shift introduced that translates the metal rod linearly to bring the chip to the center of the glass cell.

see section 2.4. With the high intensity and the mixed polarization of the MOT beams there is a high probability of an off-resonant excitation of the $F=2 \rightarrow F^{\prime}=2$ transition. The $F^{\prime}=2$ state can decay by spontaneous emission into the lower ground state $(F=1)$, which is dark. A re-pump laser is required to prevent all the atoms being rapidly pumped into this state and lost from the trap. This additional laser is tuned to the $F=1 \rightarrow F^{\prime}=2$ transition as shown in Figure 22.

To extract the properties of the atom cloud it must be imaged. For this we will use the imaging beam, this imaging beam is resonant with the $F=2 \rightarrow F^{\prime}=3$ transition. There are many ways to get these frequencies; in the different atomic experiments around the world we can find a full variety of schemes [81] [82] [83].

In the present experiment we will focus on the laser system of the running microBEC experiment at LENS, a picture of which is reproduced in Figure 23. This experiment contained Magnetic fields sources, RF antennas, optical structures, all integrated in a micro-chip with a Bose-Einstein condensate far few hundred microns from the surface of this Atom chip. The benefits are higher trap frequencies, shorter evaporation time and 


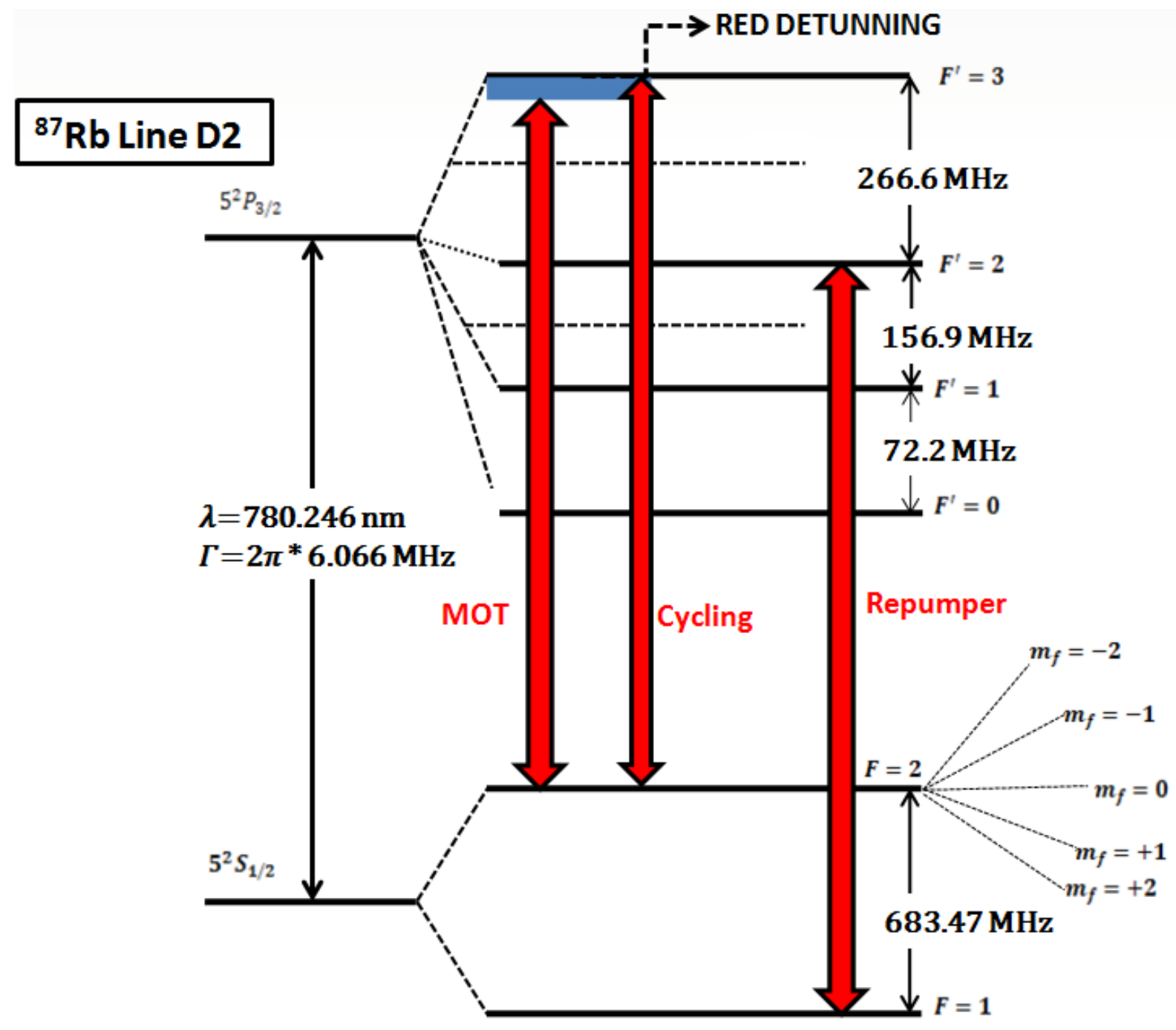

Figure 22: The hyperfine structure of the $5 s^{2} S_{\frac{1}{2}}$ and $5 p^{2} S_{\frac{3}{2}}$ states of ${ }^{87} \mathrm{Rb} \mathrm{D} 2$ line, intervals (in $\mathrm{MHz}$ ) between the hyperfine levels. The transition used for MOT/molasses cooling, imaging/cycling and re-pumper are indicated (red arrows) (Figure regenerated from 66). 


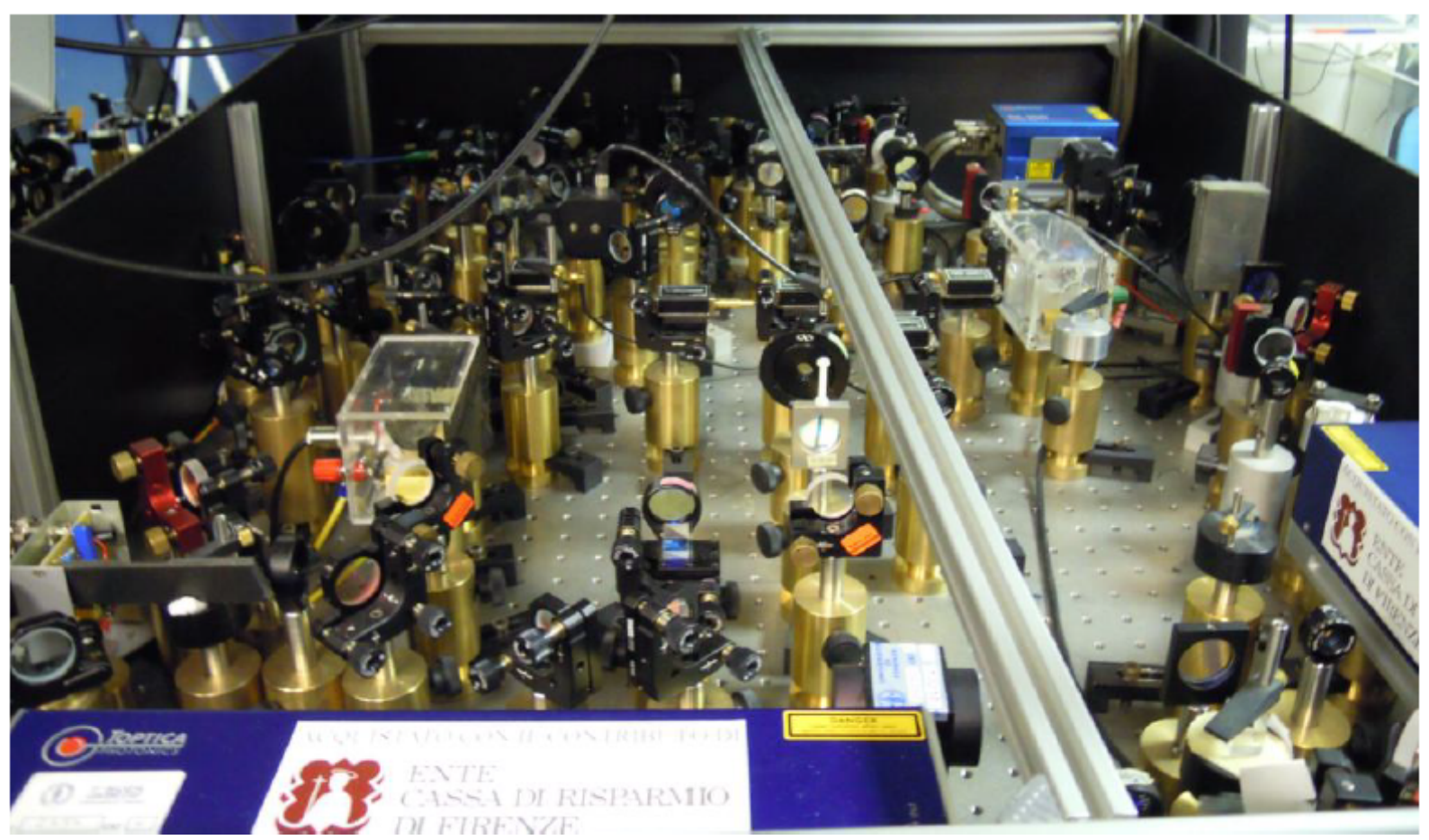

Figure 23: Photo of the laser-system box, taken from 66

better atom-field coupling. The goal of this experiment is to make the best use of Atom chips for quantum technological applications. A detailed description of the experimental apparatus plus experimental results can be found in the $\mathrm{PhD}$ thesis of Ivan Herrera [66. Thesis [66] also gives a detailed description of laser system and BEC production.

From the lasers box the light beams go to the science cell on the optical table. Figure 24 shows the optical setup of this part. A PBS cleans the polarization at the output of the cooling light optical fiber that is then split into the four beams required for the mirror MOT. Two pair of lenses in a telescope configuration enlarge the beams to use the entire chip surface. Finally the beams are circularly polarized by a $\frac{\lambda}{4}$ waveplate. Each beam has a $e^{-2}$ beam diameter of $10-12 \mathrm{~mm}$ and a power of about $\sim 70 \mathrm{~mW}$ or $\sim 10 \mathrm{~mW} / \mathrm{cm}^{2}$ intensity. The MOT re-pumping beam, with the total power of $7 \mathrm{~mW}$ after the fiber, is mixed with the cooling light in the first of the splitting PBS cubes. 


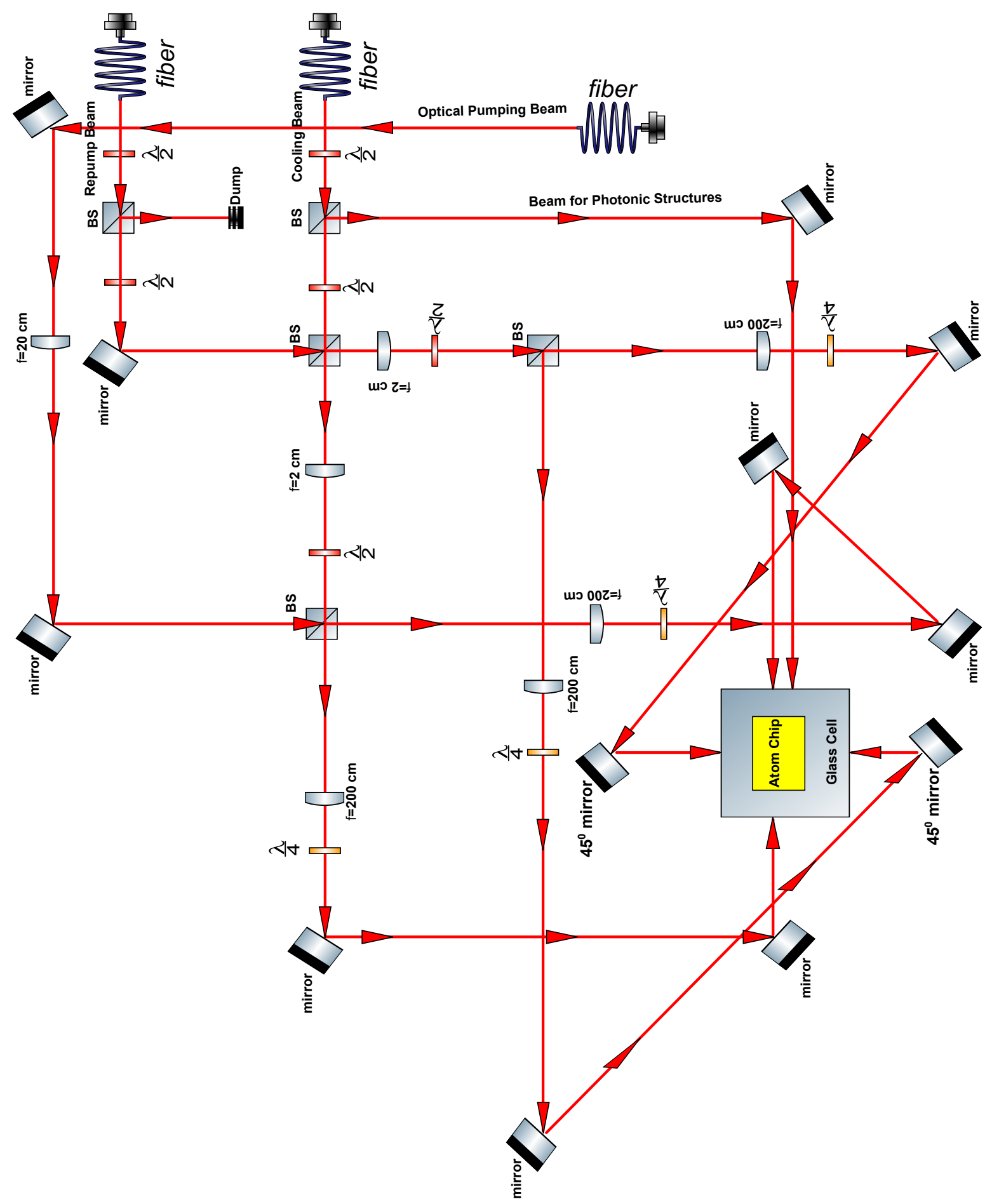

Figure 24: Main system as used by the microBEC experiment [66]. Figure is showing the optics that is to serve as a prototype of the new experiment projected in the present work. 


\subsection{BIAS and Anti-Helmholtz Coils}

\section{Magnetic field expression for rectangular current loop:}

When a current is passed through a conducting wire it contributes to form a magnetic field at the observation point $r$. The Biot-Savart Law gives us the relationship between the magnetic field and its source current. The Biot-Savart Law is given by [84] [85].

$$
d B=\frac{\mu_{o}}{4 \pi} \frac{I d I \times \hat{r}}{|r|^{2}}
$$

where,

$d I=$ infinitesimal length vector of the current source element carrying electric current I $\hat{r}=$ unit vector to specify the direction of the displacement vector $\mathrm{r}$ from the current source segment to the field point

$\mu_{o}=4 \pi \times 10^{-7} N A^{-2}=$ vacuum permeability

\section{Helmholtz Coils:}

Helmholtz coils are used for producing a region of nearly uniform magnetic field. They consist of two identical rectangular magnetic coils (solenoids) that are placed symmetrically along a common axis, one on each side of the experimental area, separated by a distance $d$ and the radius $r$ of the coil. Each coil carries an equal electric current in the same direction. The magnetic field on the axis of a rectangular current loop of radius $r$ carrying a steady current $I$ is shown in Figure 25 86.

When current $I$ is passed through the wires then the magnetic induction on $E$ is calculated in

$$
\vec{H}=\frac{\mu_{\circ} I}{2 \pi r} \frac{\cos \theta_{1}+\cos \theta_{2}}{2} \vec{H}_{\circ}
$$

Where,

$\theta_{1}=$ Angle of $\angle E L M$

$\theta_{2}=$ Angle of $\angle E M L$

$r=$ Orthogonal distance from $E$ to $L M$ 


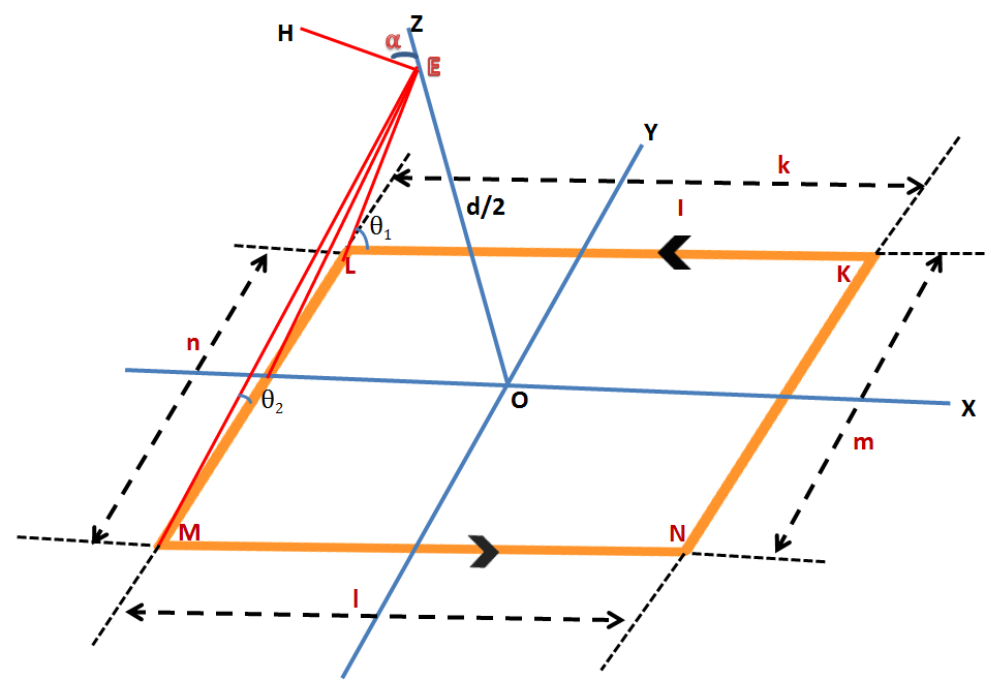

Figure 25: Model of the one rectangular coil, showing the 3-D coordinates (X, Y, Z) and L, M, $\mathrm{N}, \mathrm{K}$ are the corners of the rectangular coil with length $\mathrm{l}, \mathrm{m}, \mathrm{n}, \mathrm{k}$ respectively [86].

$\vec{H}_{\circ}=$ Unit vector of $\vec{H}$

Figure 25 also shows angle $\alpha$, the angle between $\vec{H}_{\circ}$ and z-axis. The value of $\alpha$ can be calculated by

$$
\cos \alpha=\frac{\frac{l}{2}}{\sqrt{\left(\frac{l}{2}\right)^{2}+\left(\frac{d}{2}\right)^{2}}}=\frac{l}{\sqrt{l^{2}+d^{2}}}
$$

From Equation 3.2 and 3.3 , the magnetic induction $\vec{H}_{L M}$ on $E$ generated by current $L M$ is given as 86

$$
\vec{H}_{L M}=\frac{\mu_{\circ} I}{\pi} \frac{1}{\sqrt{l^{2}+d^{2}}} \frac{m}{\sqrt{l^{2}+m^{2}+d^{2}}} \vec{H}_{L M_{\circ}}
$$

$\vec{H}_{L M \circ}$ is the unit vector of $\vec{H}_{L M}$. If $H_{z L M}$ is the z-component of $\vec{H}_{L M}$, then

$$
H_{z L M}=\left|\vec{H}_{L M}\right| \cos \alpha=\left|\vec{H}_{L M}\right| \frac{l}{\sqrt{l^{2}+d^{2}}}=\frac{\mu_{\circ} I}{\pi} \frac{l m}{\left(l^{2}+d^{2}\right) \sqrt{l^{2}+m^{2}+d^{2}}}
$$

Also for z-component of $H_{M N}, H_{M K}$ and $H_{K L}$

$$
\begin{aligned}
& H_{z L M}=H_{z N K}=\frac{\mu_{\circ} I}{\pi} \frac{l m}{\left(l^{2}+d^{2}\right) \sqrt{l^{2}+m^{2}+d^{2}}} \\
& H_{z M N}=H_{z K L}=\frac{\mu_{\circ} I}{\pi} \frac{l m}{\left(m^{2}+d^{2}\right) \sqrt{l^{2}+m^{2}+d^{2}}}
\end{aligned}
$$




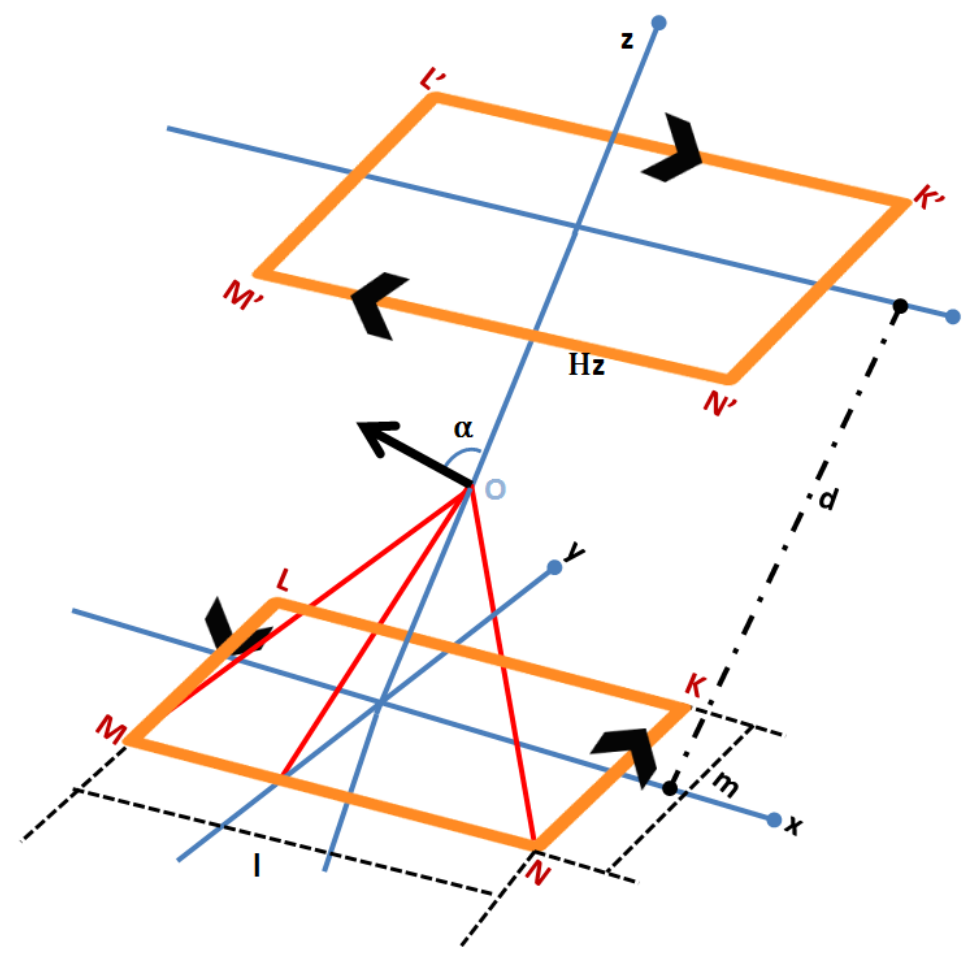

Figure 26: Model of the two rectangular coils (Figure regenerated from [86])

z-component of resultant magnetic induction on $E$ is

$$
H_{z}=\frac{\mu_{\circ} I}{\pi}\left[\frac{l m}{\left(l^{2}+d^{2}\right) \sqrt{l^{2}+m^{2}+d^{2}}}+\frac{l m}{\left(m^{2}+d^{2}\right) \sqrt{l^{2}+m^{2}+d^{2}}}\right]
$$

Until now we were dealing with the model of the single rectangular coil. Now we demonstrate the two rectangular coils in Figure 25 that is also the requirement of our experiment. A set of face-to-face $\angle M N K$ and $\angle M^{\prime} N^{\prime} K^{\prime}$ with $3-\mathrm{D}$ coordinates system are showed in Figure 26 [86. Distances between point $(0,0, \mathrm{z})$ on z-axis and from two coils $\frac{d}{2}+z$ and $\frac{d}{2}-z, H_{z}$ of two coils can be figured out by replacing the variable $(d)$ in Equation 3.8 by $\frac{d}{2}+z$. Adding two equations up, we gain the resultant magnetic induction along z-axis $H_{z}(z)$ as 86

$$
\begin{aligned}
H_{z}(z)= & \frac{2 \mu_{\circ} I}{\pi} \frac{l m}{\sqrt{l^{2}+m^{2}+(d+2 z)^{2}}}\left[\frac{1}{l^{2}+(d+2 z)^{2}}+\frac{1}{m^{2}+(d+2 z)^{2}}\right]+ \\
& \frac{2 \mu_{\circ} I}{\pi} \frac{l m}{\sqrt{l^{2}+m^{2}+(d-2 z)^{2}}}\left[\frac{1}{l^{2}+(d-2 z)^{2}}+\frac{1}{m^{2}+(d-2 z)^{2}}\right]
\end{aligned}
$$


Let's define one more variable $(A)$ as a half parameter of coil, $A=l+m$. After generalizing $l, m, d, z$ by $A$ : we get $l^{\prime}=\frac{l}{A}, m^{\prime}=\frac{m}{A}, d^{\prime}=\frac{d}{A}, z^{\prime}=z^{\prime} A$, then Equation 3.9 is presented in Equation 3.10. This is the expression of the magnetic induction on z-axis of rectangular coils set 86 .

$$
\begin{aligned}
H_{z}\left(z^{\prime}\right)= & \frac{2 \mu_{\circ} I}{\pi} \frac{l^{\prime} m^{\prime}}{A \sqrt{l^{\prime 2}+m^{\prime 2}+\left(d^{\prime}+2 z^{\prime}\right)^{2}}}\left[\frac{1}{l^{\prime 2}+\left(d^{\prime}+2 z^{\prime}\right)^{2}}+\frac{1}{m^{\prime 2}+\left(d^{\prime}+2 z^{\prime}\right)^{2}}\right]+ \\
& \frac{2 \mu_{\circ} I}{\pi} \frac{l^{\prime} m^{\prime}}{A \sqrt{l^{\prime 2}+m^{\prime 2}+\left(d^{\prime}-2 z^{\prime}\right)^{2}}}\left[\frac{1}{l^{\prime 2}+\left(d^{\prime}-2 z^{\prime}\right)^{2}}+\frac{1}{m^{\prime 2}+\left(d^{\prime}-2 z^{\prime}\right)^{2}}\right]
\end{aligned}
$$

\section{Analysis of Magnetic Field Uniformity:}

By making $H_{z}\left(z^{\prime}\right)$ more invariable around point at $z^{\prime}=0$, the magnetic field along z-axis is more uniform. Equation 3.10 giving us the values for $l^{\prime}, m^{\prime}, d^{\prime}, z^{\prime}$ but not for $A$, here $A$ tells us the effect of the invariability of magnetic field. To find $A$ and to seek for the uniformity Equation, we calculate Taylor series expansion of $H_{z}\left(z^{\prime}\right)$.

$$
H_{z}\left(z^{\prime}\right)=H_{z}(0)+H_{z}^{\prime}(0) z^{\prime}+\frac{H_{z}^{\prime \prime}(0)}{2 !} z^{\prime 2}+\frac{H_{z}^{\prime \prime \prime}(0)}{3 !} z^{\prime 3}+O\left(z^{\prime 4}\right)
$$

$O\left(z^{\prime 4}\right)$ is the fourth order infinitesimal because $H_{z}\left(z^{\prime}\right)$ is even function, then we get

$$
\begin{gathered}
H_{z}^{\prime}(0)=0 H_{z}^{\prime \prime \prime}(0)=0 \\
H_{z}^{\prime \prime}(0)=0
\end{gathered}
$$

Equation 3.11 , 3.12 and 3.13 , decide Equation 3.14 when $z^{\prime} \longrightarrow 0$ makes $O\left(z^{\prime 4}\right) \longrightarrow 0$

$$
H_{z}\left(z^{\prime}\right) \approx H_{z}(0), \text { when } z \longrightarrow 0
$$

In other words, $H_{z}\left(z^{\prime}\right)$ is uniform magnetic induction around $z=0$ at the level of $O\left(z^{\prime}\right) \longrightarrow 0$ when Equation 3.13 is satisfied. Therefore Equation 3.13 is the uniformity Equation. Simplified Equation 3.13 makes Equation 3.15. This is the uniformity 
Equation that we looked for

$$
B_{12} d^{\prime 12}+B_{10} d^{\prime 10}+B_{8} d^{\prime 8}+B_{6} d^{\prime 6}+B_{4} d^{4}+B_{2} d^{\prime 2}+B_{0} d^{\prime 0}=0
$$

Where,

$$
\begin{aligned}
& B_{12}=24 \\
& B_{10}=72 l^{\prime 2}+72 m^{\prime 2} \\
& B_{8}=93 l^{\prime 4}+146 l^{\prime 2} m^{\prime 2}+93 m^{\prime 4} \\
& B_{6}=72 l^{\prime 6}+104 l^{\prime 4} m^{\prime 2}+104 l^{\prime 2} m^{\prime 4}+72 m^{\prime 6} \\
& B_{4}=33 l^{\prime 8}+36 l^{\prime 6} m^{\prime 2}+6 l^{\prime 4} m^{\prime 4}+36 l^{\prime 2} m^{\prime 6}+33 m^{\prime 8} \\
& B_{2}=6 l^{\prime 10}+4 l^{\prime} m^{\prime 2}-18 l^{\prime} m^{\prime 4}-18 l^{\prime} m^{\prime 6}+l^{\prime 2} m^{\prime 8}+6 m^{\prime 10} \\
& B_{0}=-2 l^{\prime 10} m^{\prime 2}-5 l^{\prime} m^{\prime 4}-6 l^{\prime 6} m^{\prime 6}-5 l^{\prime 4} m^{\prime 8}-2 l^{\prime 2} m^{\prime 10}
\end{aligned}
$$

When $l^{\prime}$ and $m^{\prime}$ are known, $d^{\prime}$ can figured out numerically with MATLAB, where only positive real solution of Equation 3.15 is meaningful [86]. Finally we used Equation 3.15 to calculate the magnetic field in anti-Helmholtz coils, these anti-Helmholtz coils will be the subject of our experiment. Further discussion of anti-Helmholtz coils and the values we used to calculate the magnetic field is described below.

\section{Anti-Helmholtz coils:}

The loading of the cold atoms into the mirror-MOT requires atom chip mirror and external $45^{\circ}$ tilted anti-Helmholtz coils pair. For trapping Rb atoms in a Magnetic Optical trap, we need a locally uniform field gradient to provide the spatially dependent Zeeman shift. To do this we run an electric current in opposite direction in Helmholtz coils that will create a magnetic field of almost zero at non vanishing gradient in the center of coils. Alkali metal MOT's have field gradients of order $10 \mathrm{G} / \mathrm{cm}$ along the symmetry direction. There is an anti-Helmholtz condition $d=\sqrt{3} R$ (where, $R$ is the radius of the coils and $d$ is the distance between center of symmetry of the fields to one coil) for which the third order derivative of its magnetic field $\frac{d^{3} B(z)}{d z^{3}}$ vanishes at the origin. Typically, one pair of anti-Helmholtz coils is wrapped around the vacuum cell in which the atoms are trapped 


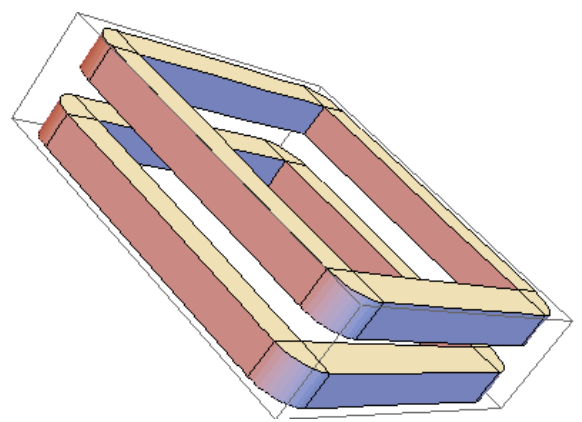

Figure 27: Anti-Helmholtz coils with distance between coils are $390 \mathrm{~mm}$, dimension of coil $160 \times 160 \mathrm{~mm}$, thickness $2 \mathrm{~mm}$, No. of windings for each coil 80, Current running in each coil is $15 \mathrm{~A}$ (note for Anti-Helmholtz coils current running in opposite direction for one coil with respect to the other coil).

and the specifies of their size depends on the experimental setup.

For our experiment we used the coordinate system in such a way that $\mathrm{z}$ denotes the symmetry axis passing through both coil centers. The diameter of the wire (used for winding the coils) is $2 \mathrm{~mm}$ (hence the final coil will have a different dimension), the distance between the two anti-Helmholtz coils $390 \mathrm{~mm}$, dimension of the coils $160 \times 160$ $\mathrm{mm}$ and the windings of the coils 80 . By running the current of $15 \mathrm{~A}$ in each wire we get the output of $8 \mathrm{G} / \mathrm{cm}$ with $70.13 \mathrm{~W}$ power consumption (per single coil) and $9.4 \mathrm{~V}$ voltage drop across each coil. Figure 27 is showing the rectangular coils, Figure 28 show's the simulation result of the coils that were generated in MATLAB. Table 1 shows the specific values of the rectangular coils that are generated in Mathematica.

\begin{tabular}{|l||l|l|l|l|l|l|l|l|}
\hline Coil & Current & $\begin{array}{l}\text { No. of } \\
\text { windings }\end{array}$ & $\begin{array}{l}\text { Dimension } \\
\text { of the } \\
\text { coil }\end{array}$ & $\begin{array}{l}\text { Diameter } \\
\text { of wire }\end{array}$ & $\begin{array}{l}\text { Distance } \\
\text { between } \\
\text { coils }\end{array}$ & $\begin{array}{l}\text { Magnetic } \\
\text { field } \\
\text { generated }\end{array}$ & $\begin{array}{l}\text { Power } \\
\text { consump- } \\
\text { tion per } \\
\text { single coil }\end{array}$ & $\begin{array}{l}\text { Voltage } \\
\text { drops } \\
\text { across } \\
\text { both coils }\end{array}$ \\
\hline Rectangle & $15 \mathrm{~A}$ & 80 & $\begin{array}{l}160 \times 160 \\
\mathrm{~mm}\end{array}$ & $2 \mathrm{~mm}$ & $390 \mathrm{~mm}$ & $8 \mathrm{G} / \mathrm{cm}$ & $70.13 \mathrm{~W}$ & $9.35 \mathrm{~V}$ \\
\hline
\end{tabular}

Table 1: The coordinate system is chosen such that $\mathrm{z}$ denotes the symmetry axis passing through both coil center

\section{BIAS Coils:}

During the different stages of the cooling cycle, one applies external bias fields generated by further magnetic coils. The external magnetic bias fields are also used to manipulate the 


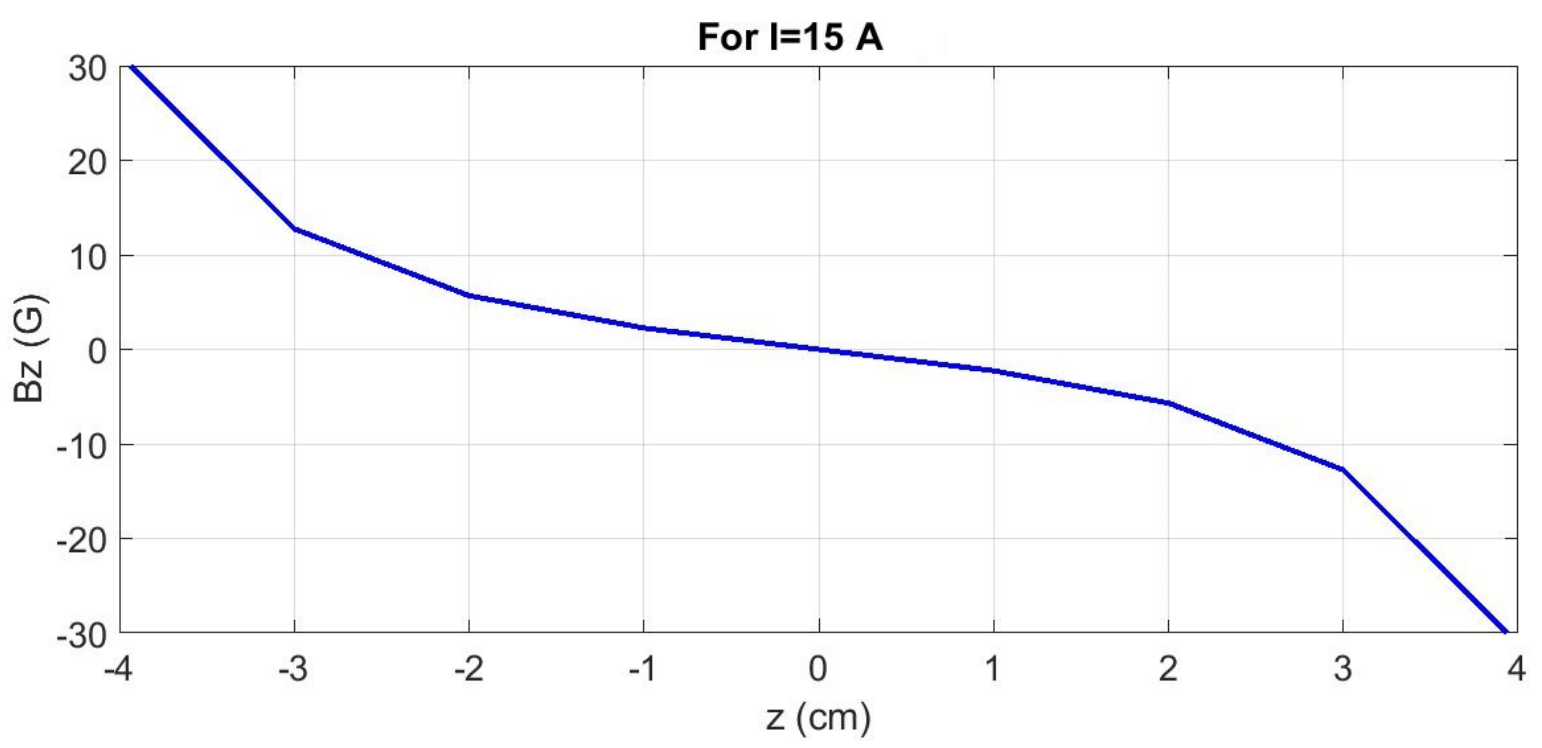

Figure 28: Plot showing the magnetic field is zero at the center and linearly increasing from center.

cold atoms position and level structure. Therefore, the BIAS coils play a very important role in the entire atom-chip system.

To design the magnetic coils five points have to be taken into account:

- The limited space of the system. All the coils have to share the space with the laser beams and the vacuum connection to the cell [66].

- The intensity of the field, the minimum gradient from the MOT coils is set around 9 Gauss/cm and a maximum bias field of 60 Gauss, in the x-direction is needed for high compressions in the magnetic micro trap [66].

- Minimizing the heating of the coils during the cooling cycle is essential to make the system stable. For this the resistance of the coils has to be as low as possible [66.

- Homogeneity of the fields in space is required. To prevent the atomic cloud from moving away during the different phases of the experiment, it is essential to minimize the gradients for the bias coils. And the quadrupole should not suffer deformations from the loading point to the loading of the magnetic trap. One needs bigger and round coils to do this, and to try to maintain the Helmholtz condition for the 
distance between them, or the anti-Helmholtz condition in the case of the quadrupole coils [66].

- Minimizing the inductance of the coils is another important point. Low inductance allow for a fast switching of the magnetic field. To improve this point one has to reduce the numbers of turns per coil and reduce to the diameter of the coils [66].

For our experiment we designed and simulate three BIAS coils named $I B_{x}, I B_{y}$ and $I B_{z}$, respectively.

$\mathrm{IB}_{x}, \mathrm{IB}_{y}$ and $\mathrm{IB}_{z}$ :

The design of $I B_{x}, I B_{y}$ and $I B_{z}$ coils are shown in Figure 29, in which 1 A current run through all the coils. The distance between the coils are $106.6 \mathrm{~mm}\left(I B_{x}\right), 89.2 \mathrm{~mm}\left(I B_{y}\right)$ and $49.4 \mathrm{~mm}\left(I B_{z}\right)$ respectively. The thickness of the coils are $11.7 \mathrm{~mm}\left(I B_{x}\right), 10.4 \mathrm{~mm}$ $\left(I B_{y}\right)$ and $7.8 \mathrm{~mm}\left(I B_{z}\right)$. The dimensions of both coils are $95.4 \times 45.4$ for $\left(I B_{x}\right), 93.8 \times$ 57.8 for $\left(I B_{y}\right)$ and $135.6 \times 135.6$ for $\left(I B_{z}\right)$. Number of windings are $81\left(I B_{X}\right), 64\left(I B_{y}\right)$ and $36\left(I B_{z}\right)$. When 1 A current run in coils $\left(I B_{x}, I B_{y}, I B_{z}\right)$ we get the best result (14, $5.5,5.5)$ for $\left(I B_{x}\right),(10,6.2,6.2)$ for $\left(I B_{y}\right)$ and $(13,5.3,5.3)$ for $\left(I B_{z}\right)$. Power consumption per single coil $0.34 \mathrm{~W}\left(I B_{x}\right), 0.26 \mathrm{~W}\left(I B_{y}\right)$ and $0.26 \mathrm{~W}\left(I B_{z}\right)$. The voltage drop across both coils $0.67 \mathrm{~V}\left(I B_{x}\right), 0.53 \mathrm{~V}\left(I B_{y}\right)$ and $0.52 \mathrm{~V}\left(I B_{z}\right)$. Graph generated of all these values in Mathematica is shown in Figure 29. Design and simulation were aided by the program Radia 87] due to its capacity to simulate magnetic fields and at the same time graph a 3D representation of the objects. For simplicity and for better understanding we put all the values and result in Table 2. Table 2 shows the values for $1.3 \mathrm{~mm}$ diameter thickness of coil. Our whole set-up with MOT beams, image beams, UHV glass cell, BIAS coils, Helmholtz coils, gate valve, four cross pipe and liner motion stage is shown in Figure 30. 

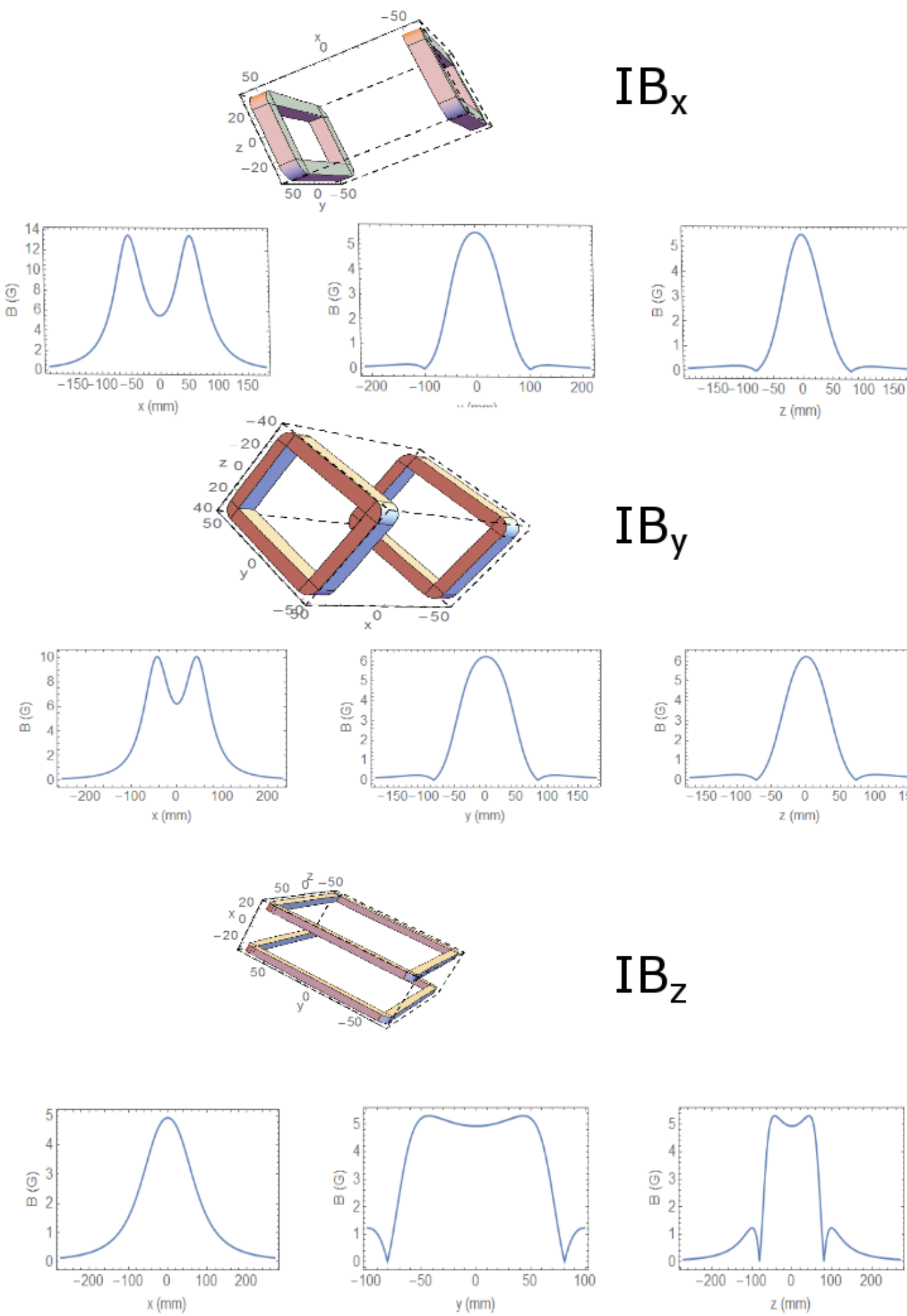

Figure 29: $3 \mathrm{D}$ representation of BIAS coils and their plots for $I B_{x}, I B_{y}$ and $I B_{z}$. 


\begin{tabular}{|l||l|l|l|l|l|l|l|l|}
\hline Coil & Current & Windings & $\begin{array}{l}\text { Coil Dimen- } \\
\text { sions (mm) }\end{array}$ & $\begin{array}{l}\text { Diameter } \\
\text { of coils } \\
(\mathrm{mm})\end{array}$ & $\begin{array}{l}\text { Distance } \\
\text { between } \\
\text { coils (mm) }\end{array}$ & $\begin{array}{l}\text { Power } \\
\text { consump- } \\
\text { tion per } \\
\text { single coil } \\
\text { ch) }\end{array}$ & $\begin{array}{l}\text { Voltage } \\
\text { drops } \\
\text { across } \\
\text { both coils } \\
(\mathrm{V})\end{array}$ & $\begin{array}{l}\text { BIAS } \\
\text { Field } \\
\mathrm{B}(\mathrm{G})\end{array}$ \\
\hline$I B_{x}$ & $1 \mathrm{~A}$ & 81 & $95.4 \times 45.4$ & 11.7 & 106.6 & 0.34 & 0.67 & 5.5 \\
$I B_{y}$ & $1 \mathrm{~A}$ & 64 & $83.8 \times 57.8$ & 10.4 & 89.2 & 0.26 & 0.53 & 6.2 \\
$I B_{z}$ & $1 \mathrm{~A}$ & 36 & $135.6 \times 135.6$ & 7.8 & 49.4 & 0.26 & 0.52 & 4.9 \\
\hline
\end{tabular}

Table 2: BIAS field for IBx, IBy and IBz. These values are for $1.3 \mathrm{~mm}$ thickness wire

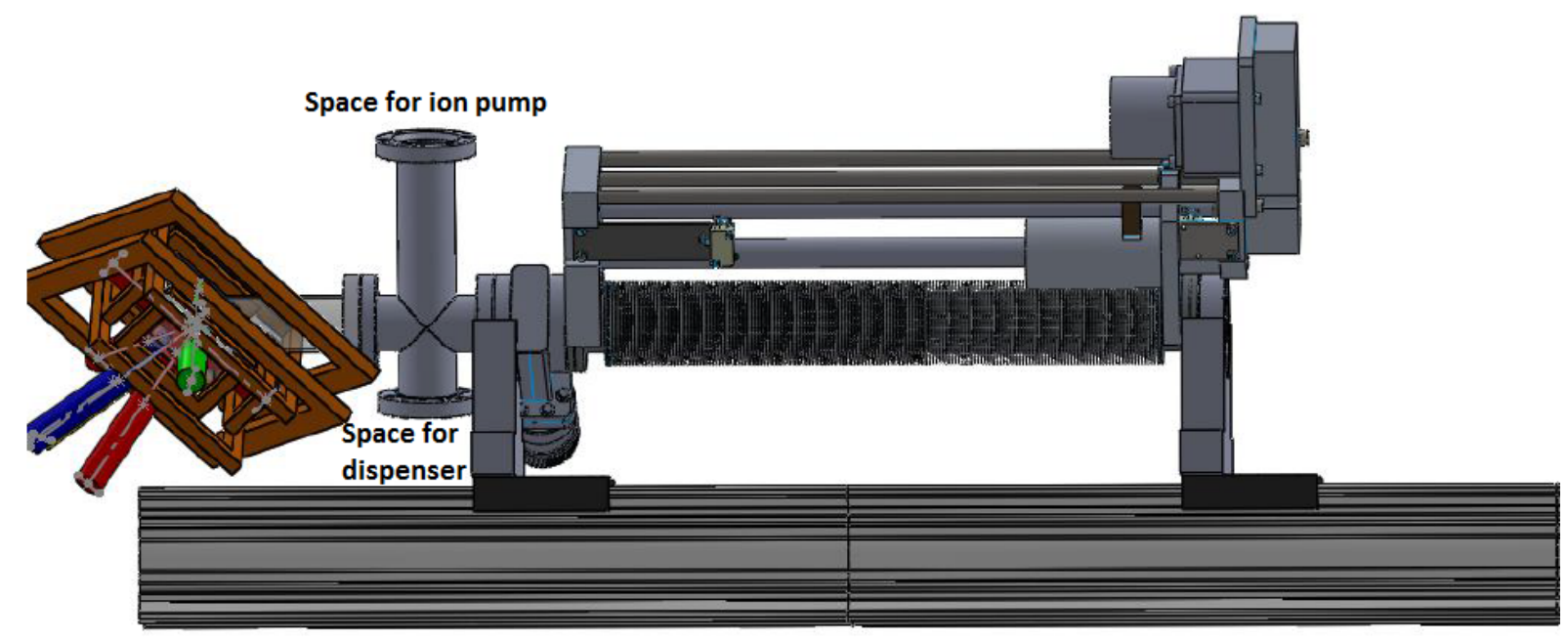

Figure 30: Whole set-up with optical access in the presence of BIAS and Helmholtz coils (Gate valve to be used in the experiment has an additional port for the connection of a roughing pump and that this way the initial vacuum condition in the linear moving stage part will be established). Drawing of the clamps are shown in appendix 62 . 


\section{Atom Chip}

\subsection{Introduction}

While the preceding chapter treated the design of the vacuum, optical and magnetic systems, in the present and following chapters we will address the issue of the Atom Chip that will be the central scientific component of the projected experiment. The current chapter will give a general introduction to the different techniques required for the understanding and creation of photonic structures on-chip. Then, in Chapter 5, we will address the design, simulation and fabrication of the Atom Chip for our experiment.

Moore's Law gives us a prediction of IC's operating velocities. It is predicting how structure sizes might decrease and operating velocities increase. From this it is clear that, if we want to continue following this fast progress, we will soon run into trouble as structures reach the size of single atoms. A switch to new technologies seems mandatory. So we have to think of an alternative to increase the velocities and reduce dimensions. Since last decade or so we find this alternative in the way of optical interconnects (e.g. grating couplers attached to nano-waveguides). These optical interconnects are the optimal solution that avoid the main limitations of velocities that we have in electrical interconnects [88] 89 .

To implement these optical connectors we need a solid material that should be at the highest crystal purity of any semiconductor and has the capability to produce microprocessors with millions of elements. It should be all integrated onto very small surfaces of high refractive index (i.e. $\mathrm{n} \sim 3.5$ ). This high refractive index make it possible to make micron/nano size devices on chip and to easily maintain a layer of $\mathrm{SiO}_{2}$ on top. Last but not least the cost of this material should be very low. SOI (Silicon-on-Insulator) fulfills all the above said requirements. SOI is ideal for our design and fabrication of atomic chip. It has excellent material properties that motive its use in photonics devices. Further to that these silicon wafers have high thermal conductivity, strong third-order optical non- 
linearities and high optical damage thresholds 90 .

A SOI structure consists of a very thin layer of crystalline Silicon ( $\mathrm{Si}$ ). This crystalline silicon is on an insulating layer of $\mathrm{SiO}_{2}$. SOI also features very high transparency and high refractive index due to silicon in infrared spectrum. The refractive index difference between silicon $\mathrm{n}=3.45$ (which act as a core or guided layer) and $\mathrm{SiO}_{2} \mathrm{n}=1.46$ (which acts as cladding or insulator layer) is large. For visible spectrum we have to put an extra layer of $\mathrm{SiN}$ above the $\mathrm{SiO}_{2}$ [91. These properties render SOI a perfect material for use in our atom chip.

\subsection{Silicon Photonics}

Silicon photonics is an advanced technique to create a platform to make optical devices on silicon wafer and use photon/light to transfer vast amount of data at very high speed with extremely low power. Very thin optical fibers are used for transferring data, in contrast to the use of electrical conductor wires $[92]$. The main advantage of silicon photonics is the combination of silicon manufacturing capacity with the extra advantage of optical interconnects. These optical interconnects offer a low loss platform at low cost. This low cost and high efficiency gives silicon photonics an extra edge to replace conventional metal interconnects in the future. Furthermore silicon technology opens the possibility of new applications in several different areas of optics 91.

\subsection{Nano-wire Waveguides on SOI}

This section reveals the fundamental characteristics of silicon photonics wire waveguides. Before discussing those waveguides we list the requirements to take full advantage of silicon photonics.

- Nano-wire waveguides should be constructed in silicon substrate embedded in silicon electronics devices 91. 
- Care should be taken when fabricating the waveguides that the process will not damage electronic devices. On the other hand when fabricating the electronic devices the waveguides should not be damaged [91].

- The material of waveguide must not involve any danger to silicon electronics [91].

- Both the layout of electronic circuit and photonics devices should be different and should not interfere 91$]$.

Silicon waveguides are a highly promising technology these days because of their ultrasmall geometrical structures and straightforward integrability with silicon electronics. These nano-waveguides are dielectrics based on a refractive index contrast between silicon oxide cladding and silicon core. We know from previous section that silicon has a very high refractive index which sets these nano-waveguides apart from more classic waveguide systems, e.g. optical fibers. This allows for very high intensities in the core of these nano-waveguides and facilitates the development of nonlinear properties, high confinement, small bending losses which leads to the possibility to realise waveguides with bends of just a few micron radius 91 .

\subsubsection{Propagation in Nano-waveguide}

Laser light has to propagates in these nano wire waveguides. For our current purposes it will be sufficient to treat the problem of guided wave propagation using geometrical ray optics. Snell's law represents the basics of the optical ray model. It describes a behavior of propagating waves at the surface between two media $\left(n_{1}\right.$ and $\left.n_{2}\right)$ with different refractive indexes. An electromagnetic field incident on an interface will be partially reflected and partially transmitted according to the following equation 91]:

$$
n_{1} \sin \theta_{1}=n_{2} \sin \theta_{2}
$$




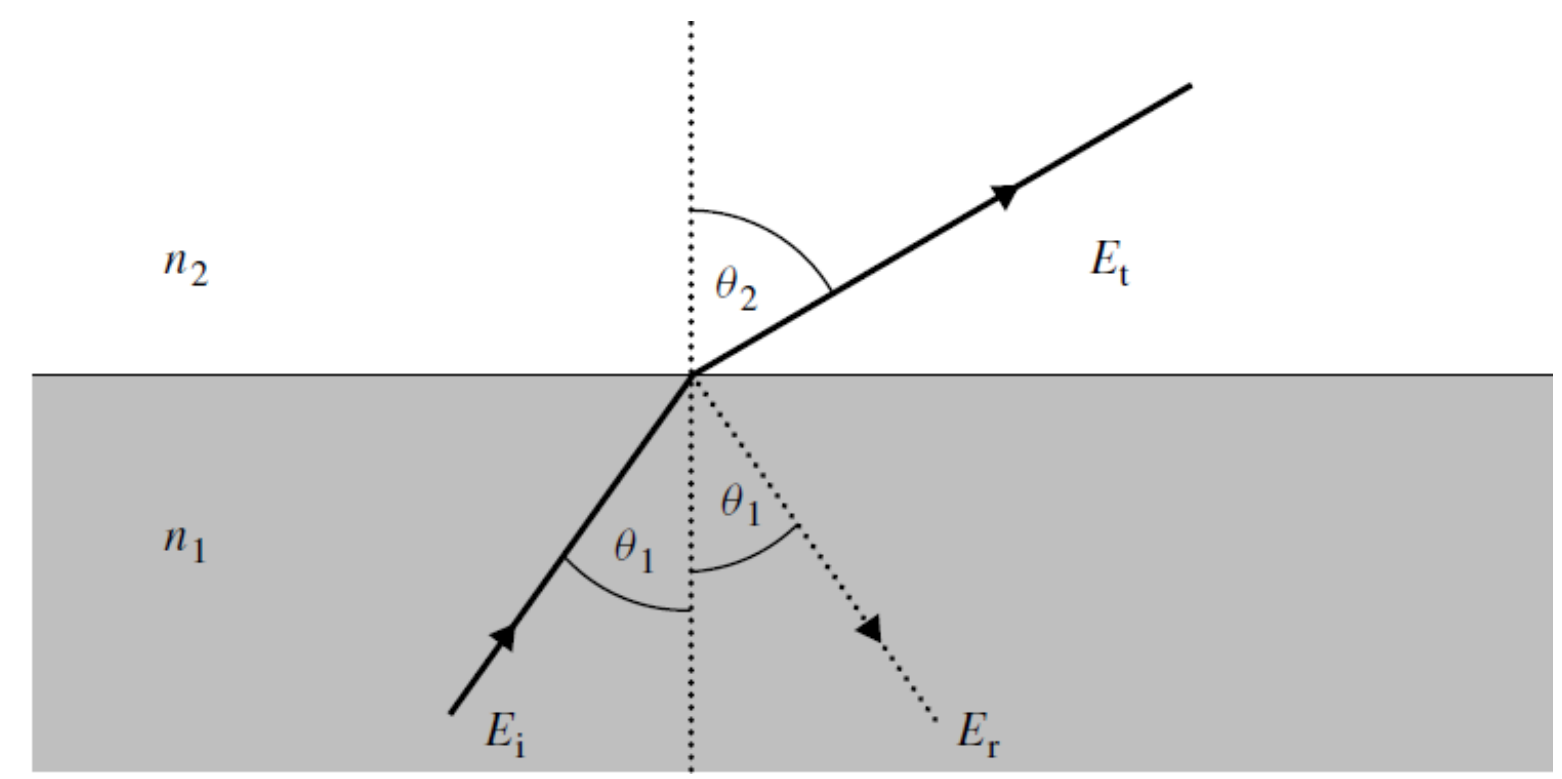

Figure 31: From medium $1\left(n_{1}\right)$ to medium $2\left(n_{2}\right)$, a ray of light $\left(E_{i}\right)$ incident on a surface, after striking on surface some of is reflected $E_{r}$ and some of it is transmitted $E_{t}$

where,

$n_{1}=$ The refractive index of medium 1

$n_{2}=$ The refractive index of medium 2

$\theta_{1}=$ Angle of Incidence

$\theta_{2}=$ Angle of refraction

Figure 31 shows the light rays refracted and reflected at the interface of two media, we should also point out that the refractive index of medium $\left(n_{1}\right)$ is greater than the refractive index of medium $\left(n_{2}\right)$ accordingly $\theta_{2}$ is greater than $\theta_{1}$. As $\theta_{1}$ is increased, $\theta_{2}$ will approach at $90^{\circ}$ angle. For some angle $\theta_{1}$, the $\theta_{2}$ will reach $90^{\circ}$, and hence Snell's law simplifies to 91 :

$$
\sin \theta_{c}=\frac{n_{2}}{n_{1}}
$$

For an angle greater than $90^{\circ}$ no light is transmitted and total internal reflection is observed, as shown in Figure 32. In nano-waveguides light experiences the same concept of total internal reflection [91]. 


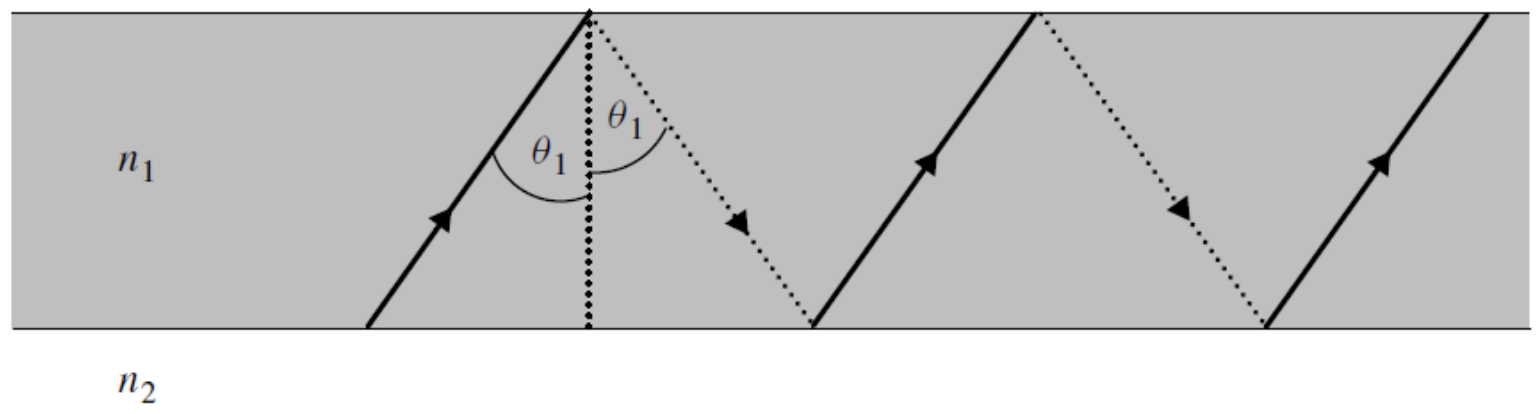

Figure 32: Total internal reflection without any part of the light being transmitted 91

\subsubsection{TE and TM polarization in Nano-waveguide}

Light can very well be polarized in an optical fiber. Indeed in experiments mostly polarization maintaining fibers are used, such that linear polarization is maintained within the fiber. Light can be polarized in TE (Transverse Electric) and TM (Transverse Magnetic) mode. TE, when electric fields of the waves are perpendicular to the plane of incidence. TM, when magnetic fields are parallel to the plane of incidence.

The Fresnel formula 91$]$ describes the reflection coefficients $r_{T E}$ and $r_{T M}$, that can usually be written for TE polarization as

$$
r_{T E}=\frac{n_{1} \cos \theta_{1}-n_{2} \cos \theta_{2}}{n_{1} \cos \theta_{1}+n_{2} \cos \theta_{2}}
$$

and for TM polarization

$$
r_{T M}=\frac{n_{1} \cos \theta_{1}-n_{2} \cos \theta_{2}}{n_{1} \cos \theta_{1}+n_{2} \cos \theta_{2}}
$$

By using Snell's law and after all calculations both equations are written as:

$$
\begin{gathered}
r_{T E}=\frac{n_{1} \cos \theta_{1}-\sqrt{n_{2}^{2}-n_{1}^{2} \sin ^{2} \theta_{1}}}{n_{1} \cos \theta_{1}+\sqrt{n_{2}^{2}-n_{1}^{2} \sin ^{2} \theta_{1}}} \\
r_{T M}=\frac{n_{2}^{2} \cos \theta_{1}-n_{1} \sqrt{n_{2}^{2}-n_{1}^{2} \sin ^{2} \theta_{1}}}{n_{2}^{2} \cos \theta_{1}+n_{1} \sqrt{n_{2}^{2}-n_{1}^{2} \sin ^{2} \theta_{1}}}
\end{gathered}
$$

When the angle of incidence is less than $90^{\circ}$ partial reflection occurs and the relative coefficient is real. When it exceeds $90^{\circ}$ angle we have total internal reflection. the magnitude 
of $r$ is

$$
|r|=1
$$

Here $r$ can be both real and complex, this means that phase shift is inflicted on the reflected wave. We can now denote this as

$$
r=\exp (j \phi)
$$

and hence $\phi_{T E}$ and $\phi_{T M}$ is given by

$$
\begin{aligned}
& \phi_{T E}=2 \tan ^{-1} \frac{\sqrt{\sin ^{2} \theta_{1}-\left(\frac{n_{2}}{n_{1}}\right)^{2}}}{\cos \theta_{1}}, \\
& \phi_{T M}=2 \tan ^{-1} \frac{\sqrt{\frac{n_{1}^{2}}{n_{2}^{2}} \sin ^{2} \theta_{1}-1}}{\frac{n_{2}}{n_{1}} \cos \theta_{1}} .
\end{aligned}
$$

\subsubsection{TM and TE modes in Nano-waveguide:}

We will now enhance our optical ray model for a better understanding of the propagation of waves in nano-waveguides. Let's consider briefly propagation of an electromagnetic wave in analogy to electrical circuit theory. We separately write the electric and magnetic fields 91$]$ :

$$
\begin{aligned}
& E=E_{\circ} \exp [j(k z \pm \omega t)] \\
& H=H_{\circ} \exp [j(k z \pm \omega t)]
\end{aligned}
$$

Here $z$ represents the direction of propagation and the phase of the wave is

$$
\phi=k z \pm \omega t
$$

This phase wave varies with time and with distance $z$. By taking the time derivative it is represented as:

$$
\left|\frac{\partial \phi}{\partial t}\right|=\omega=2 \pi f
$$


Where,

$\omega=$ Angular frequency in $\mathrm{rad} / \mathrm{s}$

$f=$ frequency in $\mathrm{Hz}$

In a similar way, if we relate the phase to the propagation distance and take the derivative then the phase equation can be represented as:

$$
\frac{\partial \phi}{\partial z}=k
$$

Where,

$k=$ Wave vector or the propagation constant in the direction of the wavefront The relation of the wave vector $k$ to the wavelength is:

$$
k=\frac{2 \pi}{\lambda}
$$

This $k$ wave vector is defined in free space $k_{\circ}$ and both can be related by the refractive index of the medium $n$ :

$$
k=k_{\circ} n
$$

Now we can reproduce Figure 32 as Figure 33 with additional axis $y$ and $z$. Figure 33(a) shows the waveguide height $(h), z$ as a propagation direction and $y$ as a direction of confined light by total internal reflection. The zigzag path in Figure 33(a) shows the direction of wavenormals as the wave propagate through the waveguide and the wavevector $k=k_{\circ} n$. In Figure 33(b) we divide the wavevector into two components, $y$ and $z$ direction and we represent these by applying simple trigonometry:

$$
\begin{aligned}
& k_{z}=n_{1} k_{\circ} \sin \theta_{1} \\
& k_{y}=n_{1} k_{\circ} \cos \theta_{1}
\end{aligned}
$$




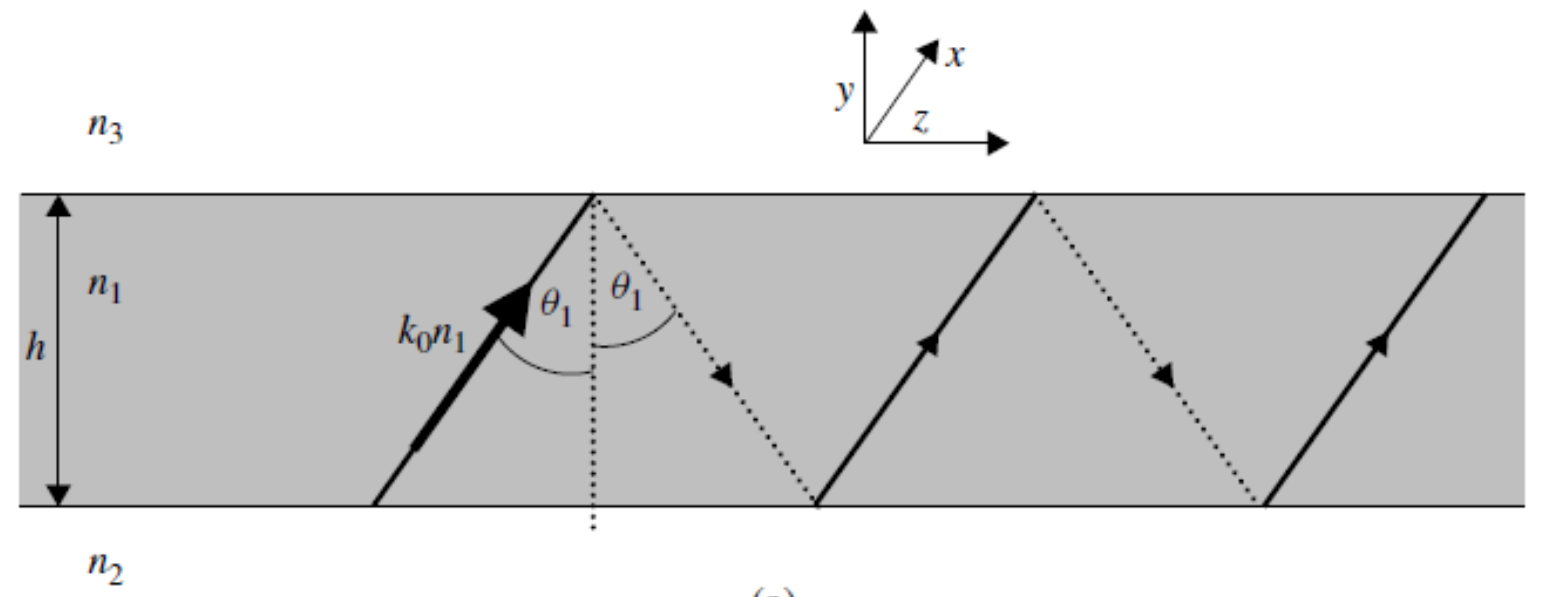

(a)

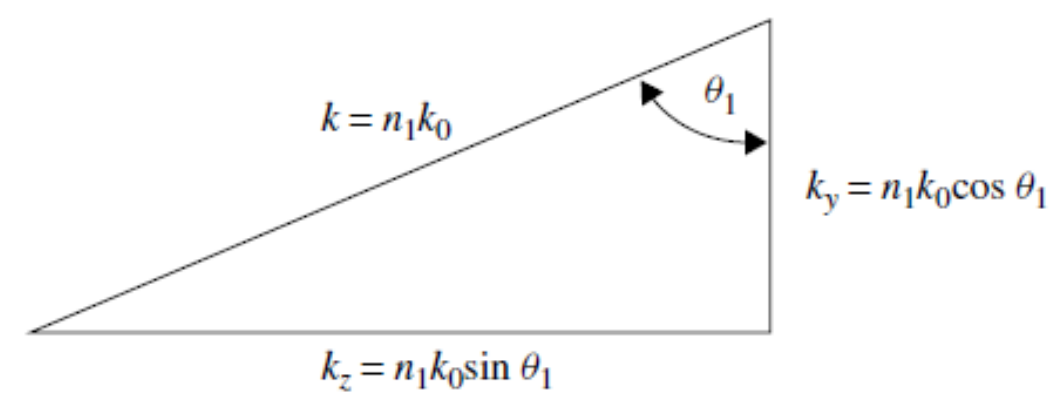

(b)

Figure 33: (a) Total internal reflection with wave vector $k_{\circ} n_{1}$ (b) $k$ vector break/ decomposed into further components $k=\left(k_{y}, k_{z}\right)$ 91. 
As we supposed that the wave is propagating in $y$ direction, it should reflect at each interface. In this way we can add up all the phase shifts acquired during a round trip. By using Equation (4.15) we introduce this phase shift as:

$$
\phi_{h}=2 k_{y} h=2 k_{\circ} n_{1} h \cos \theta_{1}
$$

We noticed in Equation 4.8 - Equation 4.10 that phase changes upon reflection at upper and lower waveguide boundaries. Introducing this reflection at upper waveguide as $\phi_{u}$ and lower waveguide as $\phi_{l}$, then our total phase shift becomes:

$$
\phi_{t}=2 k_{\circ} n_{1} \cos \theta_{1}-\phi_{u}-\phi_{l}
$$

This equation can be re-written as total phase shift that must be a multiple of $2 \pi$ :

$$
2 k_{\circ} n_{1} \cos \theta_{1}-\phi_{u}-\phi_{l}=2 m \pi
$$

Where,

$m=$ any integer

$\phi_{u}=\phi_{l}=\theta$

So the angle $\theta$ gives us a discrete set of series in Equation 4.22 and we can solve this $\theta$ corresponding to integer values of $m$. Every solution gives us the propagation constant in $y$ and $z$ direction for each polarization. Hence it will also show us that light cannot propagate in any angle $\theta$, but only in a set of discrete angles. An allowed solution is called as mode of propagation, where value of the integer $m$ represents the mode number. The first fundamental mode or TE mode is denoted as $\mathrm{TE}_{\circ}$. Similarly higher ordered modes are described using the appropriate value of $m$. Without going in detail, if $n_{2}=n_{3}$ in Figure 33 then waveguide is called symmetrical and asymmetrical if not. 


\subsubsection{Loss in Optical Nano-waveguide}

During the propagation of light in waveguides, the light wave encounters three types of losses: scattering, absorption and radiation. These three losses are dependent on the structure, material, design and fabrication of the waveguide [91].

\subsubsection{Scattering, Absorption and Radiation:}

\section{Scattering:}

Scattering in an optical waveguide is further divided into two categories: Volume and interface scattering. Volume scattering is found in the bulk waveguide material because of imperfections, contaminant atoms, or crystalline defects. Interface scattering can be found in core and cladding of waveguide due to roughness at interface. In many cases volume scattering can be neglected because prior to fabrication the waveguide material has been purified to a sufficient level. Volume scattering can be found in detail in the literature (see for example [93] [94]). In contrast, interface scattering is difficult to neglect because even after careful material fabrication losses can still be significant even for relatively smooth interfaces. The simple theory of interface scattering can be found in [95] [96]. According to [96 if an incident beam has power $P_{i}$, then the specular reflected power $P_{r}$ from a surface is given by 96 :

$$
P_{r}=P_{i} \exp \left[-\left(\frac{4 \pi \sigma n_{1}}{\lambda_{\circ}} \cos \theta_{1}\right)^{2}\right]
$$

Where,

$\sigma=$ variance of the surface roughness (or r.m.s. roughness)

$\theta_{1}=$ propagation angle within the waveguide

$n_{1}=$ refractive index of the core

From 95 we can produced the above expression for the loss coefficient due to interface 
scattering:

$$
\alpha_{s}=\frac{\cos ^{3} \theta_{1}}{2 \sin \theta_{1}}\left(\frac{4 \pi n_{1}\left(\sigma_{u}^{2}+\sigma_{l}^{2}\right)^{\frac{1}{2}}}{\lambda_{\circ}}\right)^{2}\left(\frac{1}{h+\frac{1}{k_{y u}}+\frac{1}{k_{y l}}}\right)
$$

Where,

$\sigma_{u}=$ r.m.s. roughness for the upper waveguide interface

$\sigma_{l}=$ r.m.s. roughness for the lower waveguide interface

$k_{y u}=\mathrm{y}$ direction decay constant in upper cladding

$k_{y l}=\mathrm{y}$ direction decay constant in lower cladding

$h=$ height of the waveguide

\section{Absorption:}

We distinguish three types of absorption in semiconductor nano-waveguides: band edge (interband edge) absorption, free carrier absorption and Auger recombination. Auger recombination is not very important and plays a minor role. Band edge (inter-band edge) absorption takes place when photons with energy higher than the band gap are absorbed and excite electrons from the conduction to valence band. This type of absorption can be avoided by using optical wavelengths greater then $1100 \mathrm{~nm}$, corresponding to the band gap of silicon. Unfortunately in our experiment we have to use an optical wavelength of $780 \mathrm{~nm}$, so it plays a significant role. Free carrier absorption is more significant in semiconductor waveguides. Specially also in the case of a wavelength greater then 1100 nm an electron or a hole can absorb a photon with energy lower than the band gap energy. Here transition from the valence to conducting band is not possible for an electron. These three types of absorption in semiconductor waveguides are described in Figure 34 . Theoretically these changes are well defined by the Drude Lorenz equation [97]:

$$
\Delta \alpha=\frac{e^{3} \lambda_{\circ}^{2}}{4 \pi^{2} c^{3} \epsilon_{0} n}\left(\frac{N_{e}}{\mu_{e}\left(m_{c e}^{*}\right)^{2}}+\frac{N_{h}}{\mu_{h}\left(m_{c h}^{*}\right)^{2}}\right)
$$

Where,

$e=$ electronic charge

$c=$ velocity of light in vacuum 


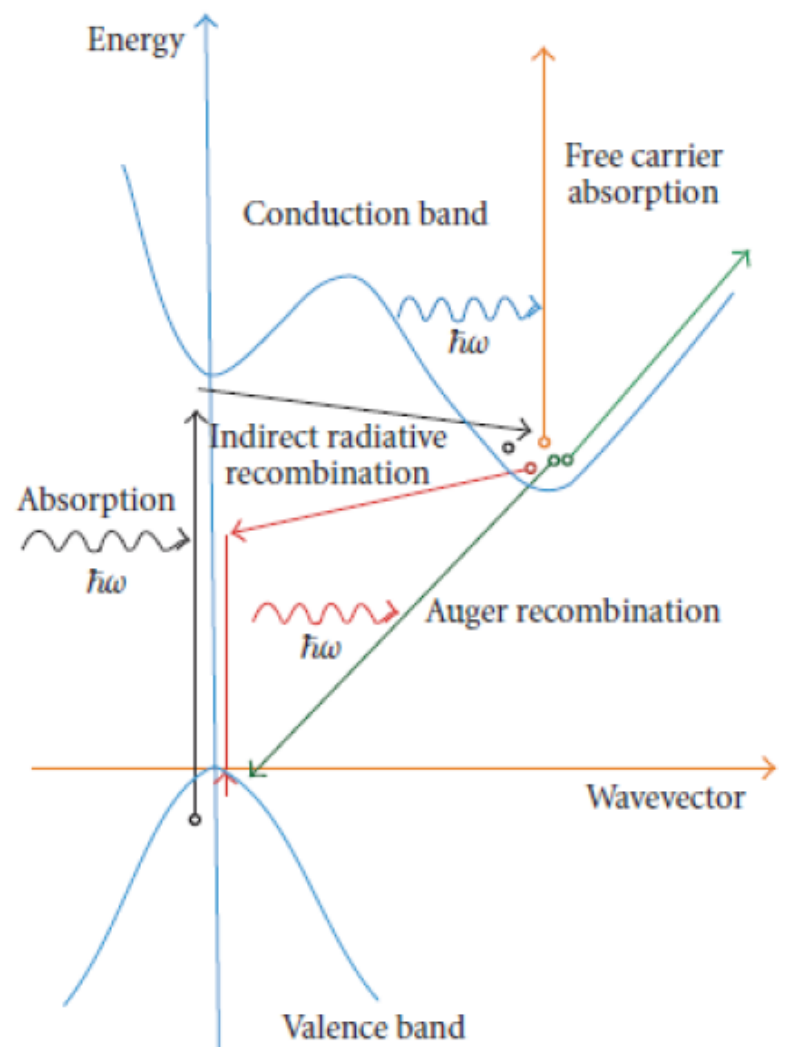

Figure 34: Valence and Conduction bands, wave vector showing horizontally with energy increasing from top to bottom vertically 97 
$\mu_{e}=$ electron mobility

$\mu_{h}=$ hole mobility

$m_{c e}^{*}=$ effective mass of electrons

$m_{c h}^{*}=$ effective mass of holes

$N_{e}=$ free electron concentration

$N_{h}=$ free hole concentration

$\epsilon_{0}=$ permittivity of free space

$\lambda_{\circ}=$ free space wavelength

\section{Radiation:}

Radiation loss in waveguides mostly depends on whether a mode within the waveguide has leakage into the surrounding media. This area is mostly in the planar region adjacent to the waveguide. Most of the time this would not happened and loss due to this effect should be negligible.

\subsubsection{Nano-waveguide Structure}

This section will give the brief intro of SOI wafer and possible waveguide structure that is exposed. When we are discussing waveguides other than optical fibers then we are bound to a three layer structure consisting of one core and two cladding regions. A typical configuration of SOI wafer is shown in Figure 35. Figure 35 shows that the silicon dioxide $\left(\mathrm{SiO}_{2}\right)$ layer is grown under the surface of the silicon wafer. Top layer is used as required by the application and known as a guiding layer. This guiding layer is typically of the order of some micrometer (vary with individual designs). After guiding the layer we have a buried oxide layer that will help to prevent the field associated with the optical modes from penetrating the silicon substrate below. The buried oxide layer is typically of half a micrometer, again individual designs vary. Two types of waveguides are widely used:

\subsubsection{Large Single Mode Waveguide}

Large single mode waveguides also known as rib or ridge waveguides are composed of a 
Figure 35: Silicon-on-Insulater (SOI) substrate fabricated with buried oxide layer and guided layer.

core and two lateral slab regions. This core and the two lateral slab regions are etched on the silicon layer. One can easily achieve this lateral confinement by the leakage of the higher order modes in these lateral slab regions. The cross section of the large single mode waveguide is shown in Figure 36. In Figure $362 a \lambda=$ core width, $2 b \lambda=$ height of the core, $2 b r \lambda=$ lateral slab regions, where $r=$ parameter that is defined as the ratio of slab to rib height. This structure has been demonstrated when the following condition is true:

$$
\frac{a}{b} \leq c+\frac{r}{\sqrt{\left(1-r^{2}\right)}}(\text { for } 0.5 \leq r \leq 1)
$$

Where,

$c=$ constant term that is 0.3 calculated in $[98]$ and again recalculated -0.05 in $[99]$ Here the term $0.5 \leq r \leq 1$ means that we restricted the rib guide from 0.5 to 1 . Which further tells us that high-order modes in vertical direction will be cut off. This is because the higher-order modes in central rib will be coupled to the fundamental mode of the slab section. This slab section mode will become leaky for $r \leq 0.5$. More regarding the condition $0.5 \leq r \leq 1$ and single-mode operation can be found in 98 . 


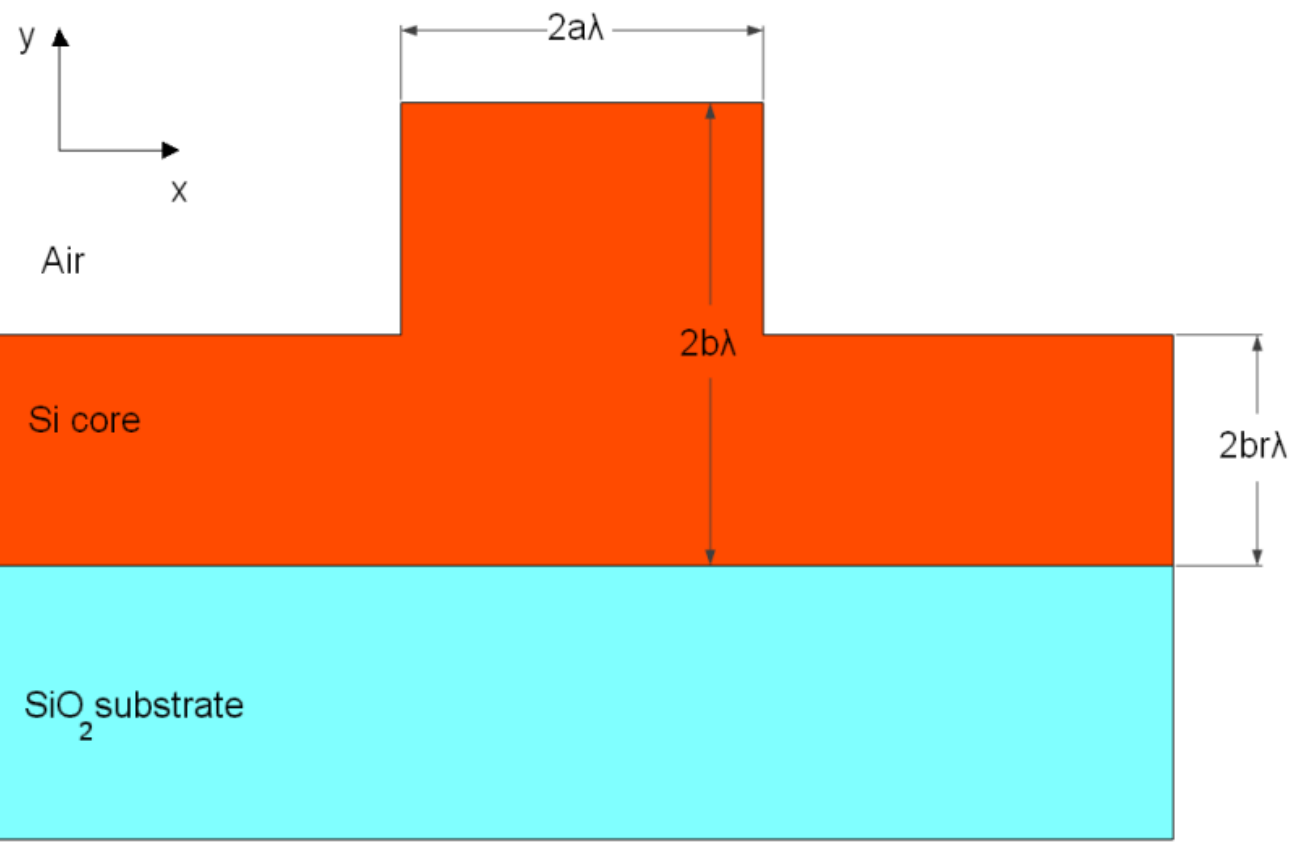

Figure 36: Sketch of a rib waveguide 98 .

\subsubsection{Strip Nano-waveguide}

A strip nano-waveguide has the dimensions in order of several nanometer. The reduction of dimensions will give us higher light confinement. The structure of a strip nano-waveguide is shown in Figure 37. The $\mathrm{SiO}_{2}$ layer thickness and width can be tuned to achieve an optimal coupling condition. This is because the input field is launched in the cladding and then couples to the core. Strip nano-waveguides do not provide us a simple single mode condition. Because of this reason several measurements and simulations are studied and have been performed in several groups. Thus the single mode condition has been found to be [101]:

$$
H<2.109^{-11.93 W}+0.7365 e^{-1.956 W}
$$

For single polarization mode, e.g. T.M and T.E, it is given by:

$$
H<63.13 e^{-23.92 W}+0.2684 e^{-0.8577 W}
$$




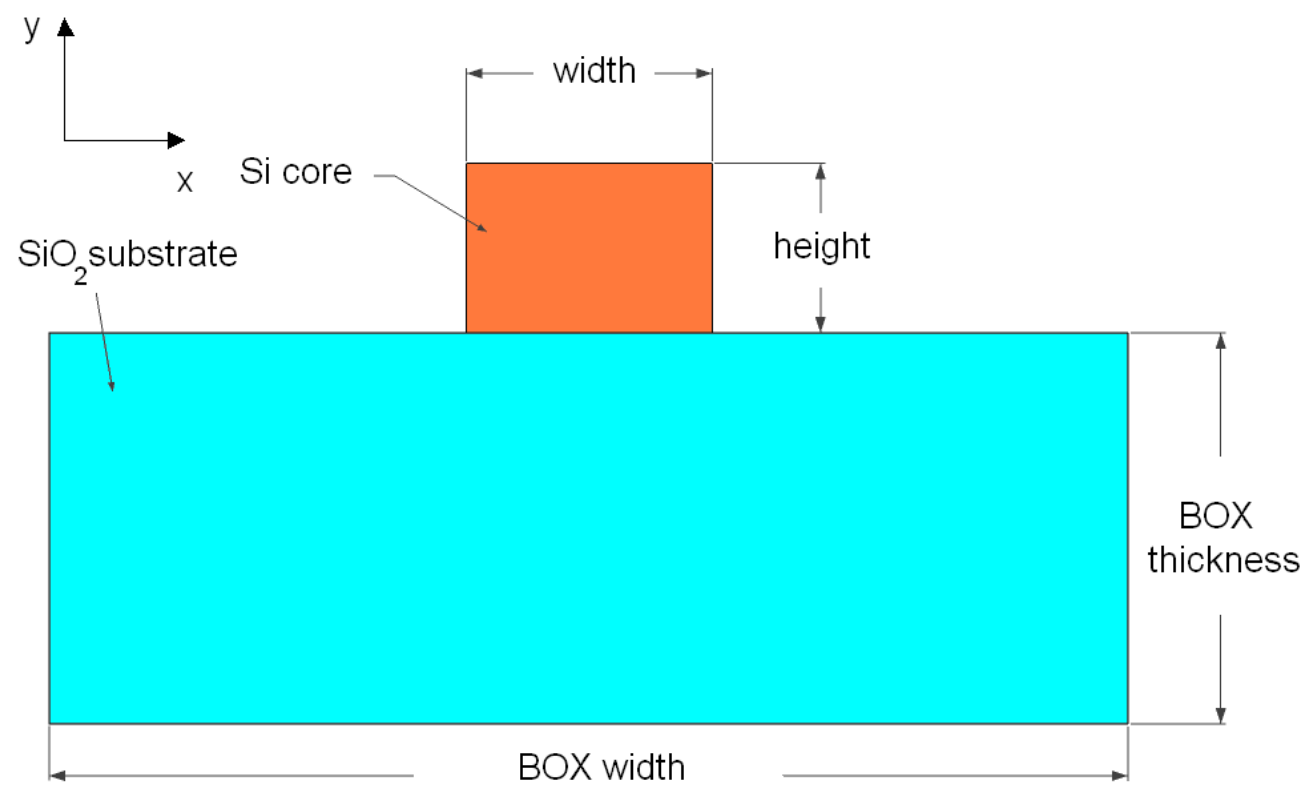

Figure 37: Structure of strip nano-waveguide 100.

Where,

$H=$ Thickness of silicon photonic wire

$W=$ Width of silicon photonic wire

Many research groups and companies have been developing optimal strip waveguide structures that give minimal propagation losses. We took some of the best result and summarized them in Table 3. We also plot the results in Figure 38.

\begin{tabular}{|l||l|l|l|l|l|}
\hline & $\begin{array}{l}\text { IBM 102 } \\
\sqrt{103}\end{array}$ & $\begin{array}{l}\text { IMEC } \\
\sqrt[104]{ }\end{array}$ & $\begin{array}{l}\text { NTT } \\
\sqrt[105]{ }\end{array}$ & $\begin{array}{l}\text { LETI } \\
\sqrt[106]{ }\end{array}$ & $\begin{array}{l}\text { Cornell } \\
\sqrt[107]{108}\end{array}$ \\
\hline Core height $(\mathrm{nm})$ & 220 & 220 & 200 & 200 & 270 \\
Core width $(\mathrm{nm})$ & 460 & 500 & 400 & 500 & 470 \\
Box thickness $(\mu \mathrm{m})$ & 2.0 & 1.0 & 3.0 & 0.7 & 3.0 \\
Loss $(\mathrm{dB} / \mathrm{cm})$ & 3.6 & 2.4 & 2.8 & 6.4 & 5.0 \\
\hline
\end{tabular}

Table 3: Strip waveguide results from different Institutions 


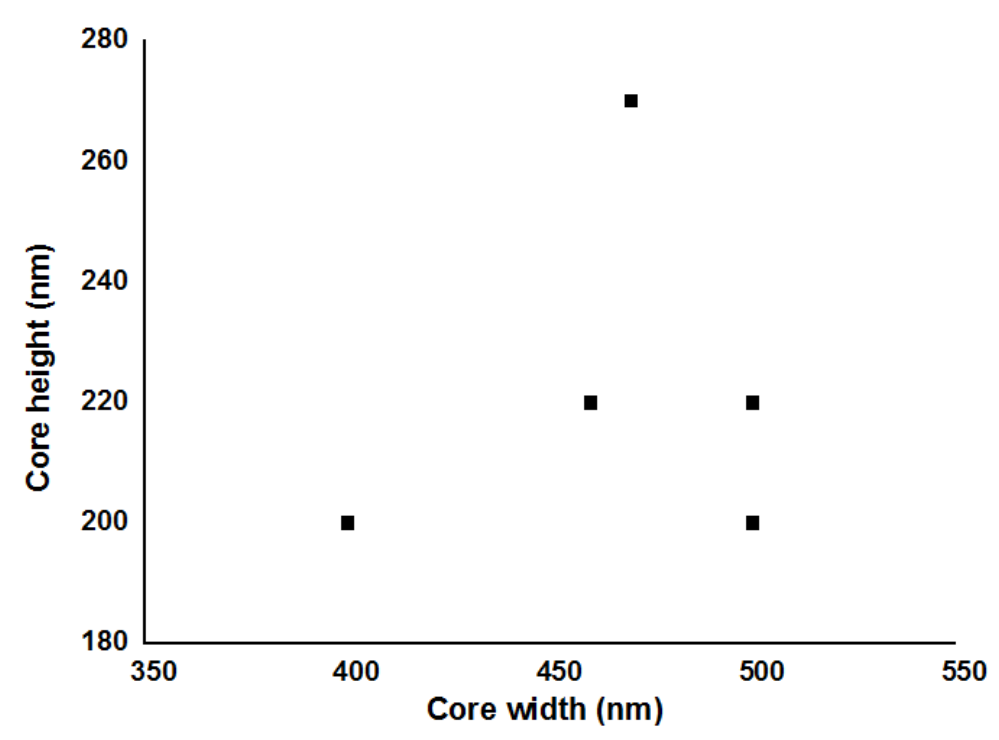

Figure 38: Graphical representation of the data given in Table 3.

\subsubsection{Coupling Light from fiber to Nano-waveguide}

Despite many positive prospects in nanophotonics, a major challenge that remains is the issue of mode mismatch between the light coming from a standard optical fiber to the light of nano-waveguides. This leads us to the mismatch between standard single mode fiber (SMF) and single mode nano-waveguide. We know that silicon nano-waveguide cross section is approximately $0.1 \mu \mathrm{m}^{2}$, with core height and width $0.4 \mu \mathrm{m}$ respectively. If we compare this values to a typical optical fiber which has a core cross sectional area of 250 $\mu m^{2}$ (i.e. radius of 8-10 $\mu m$ ) then we completely understand that optical fiber has three orders magnitude larger cross section [100]. This causes a very high amount of loss without a mode size conversion solution. A comparison of an optical fiber to a nano-waveguide is shown in Figure 39.

\subsubsection{Coupling Problem}

We summarize the main problems that arise when trying to couple light from typical fiber optics system into and out of the nano-waveguides [109].

- Large numerical aperture difference between optical fiber and nano-waveguide, that causes the lack of efficiency when coupling light out of the nano-waveguide. 


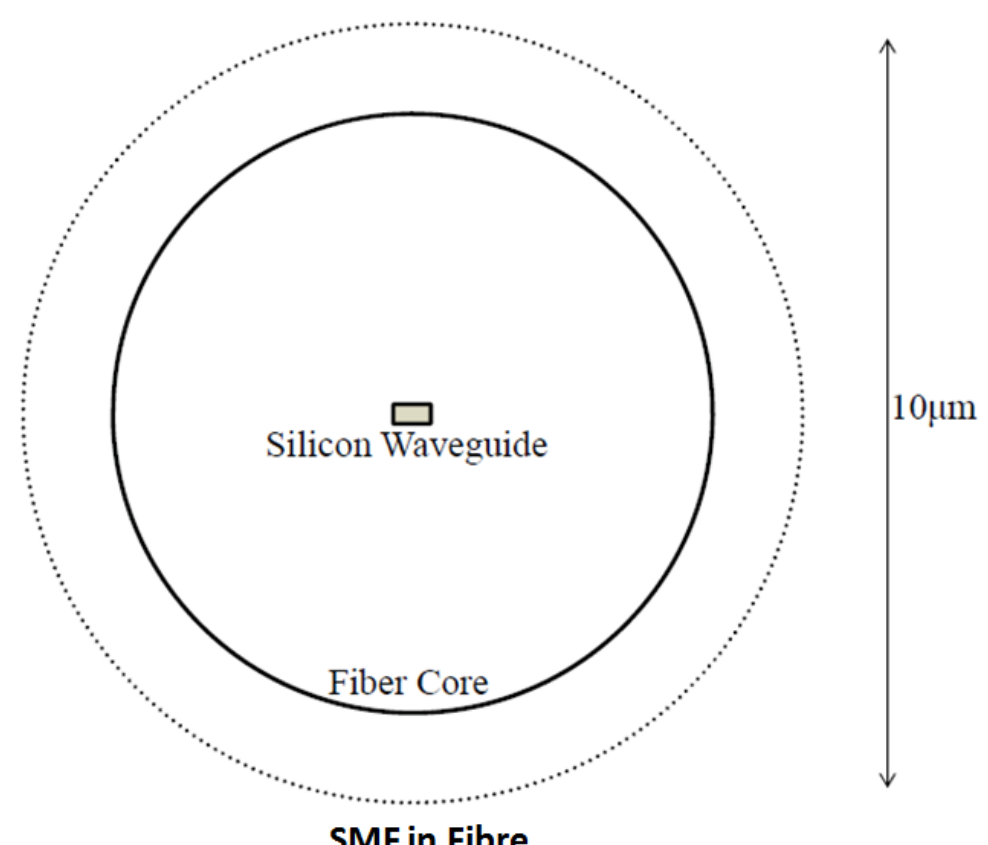

Figure 39: Comparison of nano-waveguide to laser beam diameter 109.

- The large inherent mismatch between optical fiber and nano-waveguide (i.e. 10.4 $\mu m$ for fiber and $500 \mathrm{~nm}$ by $250 \mathrm{~nm}$ rectangular nano-waveguide) shows that an enormous amount of power is lost when trying to move the signal directly from fiber to waveguide end.

- Incoming power loss can prohibit interesting nonlinearities for manifesting themselves in silicon photonics.

- When this loss is multiplied due to repeated interfaces then it can exceed the amplification offered by fiber amplifiers that make it very difficult to recover good signal from the chip.

There are many methods that try to solve the problems mentioned. Methods such as multi-dielectric structures [110], overcladdings 111], grating structures and inverse tapers have been demonstrated [112. We are going to discuss the two most important methods, to be also used in our atom chip experiment. 


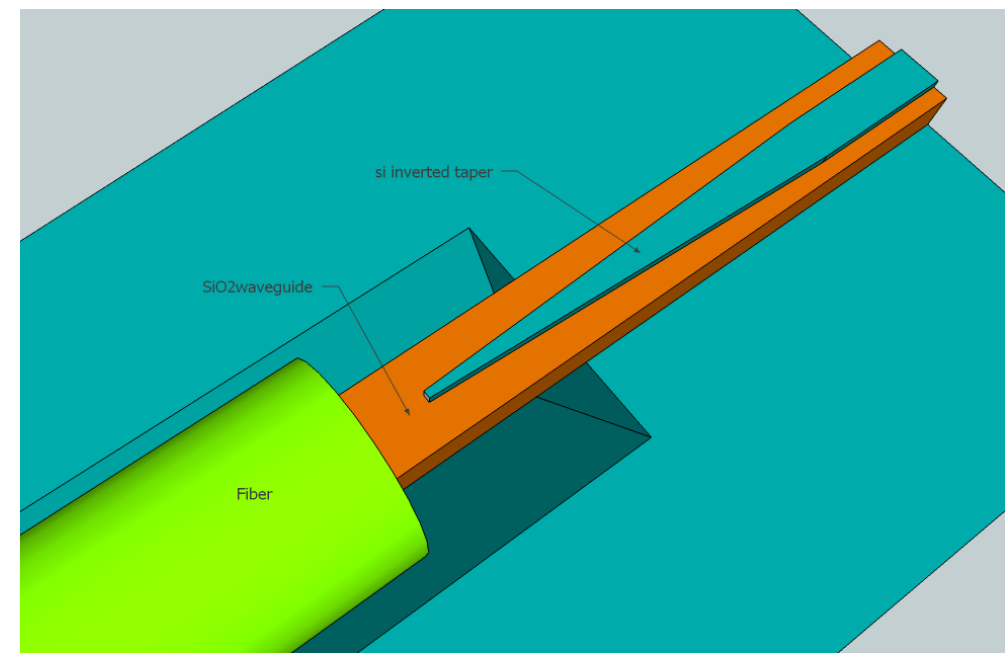

Figure 40: Silicon inverse taper on silicon dioxide nano-waveguide 100.

\subsubsection{Tapered Spot-size Convertors}

We saw that the diameter of light from standard fiber is about $10 \mu \mathrm{m}$, so one can fabricate a taper that improves the coupling efficiency by reducing the mode dimensions of the field emitted by the fiber. The taper's length should be of some millimeters to avoid excessive coupling to radiation modes in the taper. The big disadvantage of these type of tapers are strong back reflections at the facet of the couplers. Because of this reason inverse tapers are introduced. These inverse tapers from the waveguide dimensions to the dimensions of a small tip are shown in Figure 40. Further details of these inverse tapers are mentioned in 112 113.

\subsubsection{Grating-assisted Directional Coupler}

Another solution to the problem of mode matching is the use of grating couplers followed by a taper to adjust the lateral size of the incident wave-field from the fiber. Grating couplers with tapers have many advantages. These couplers are working on the principle of Bragg reflection (see also Section 4.3.8) which is giving them favorable properties. Several developments have been made and proposed to further reduce the losses in this method. Figure 41 shows a typical fiber to grating coupler coupling scheme [100. 


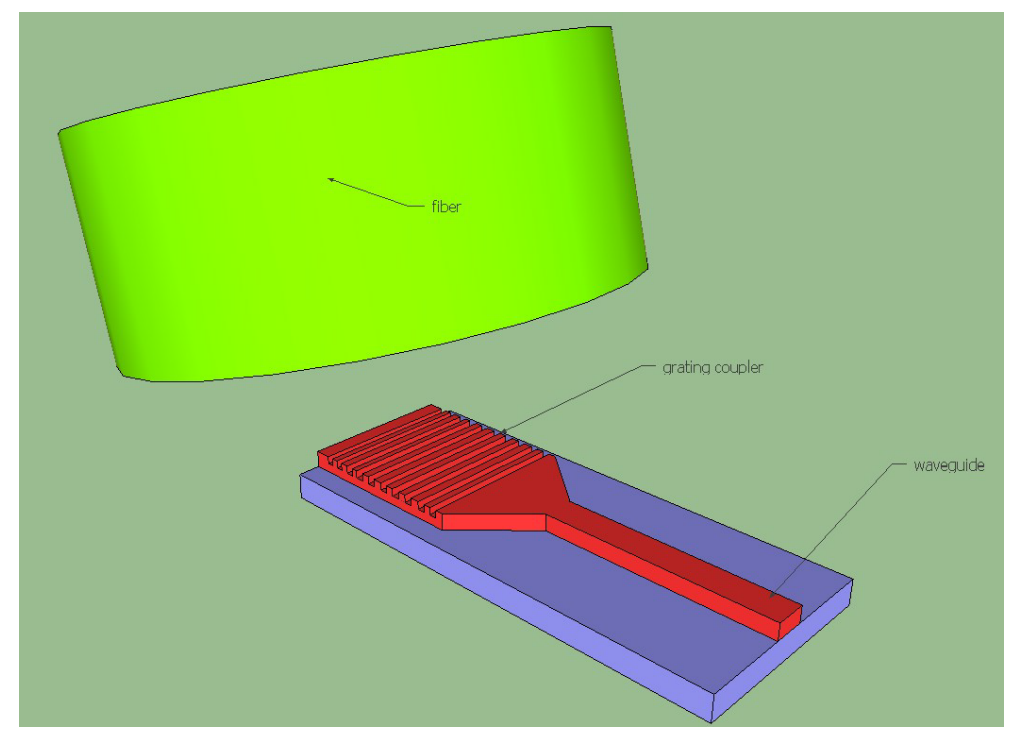

Figure 41: Grating couplers with nano-waveguide fabricated on silicon wafer 100.

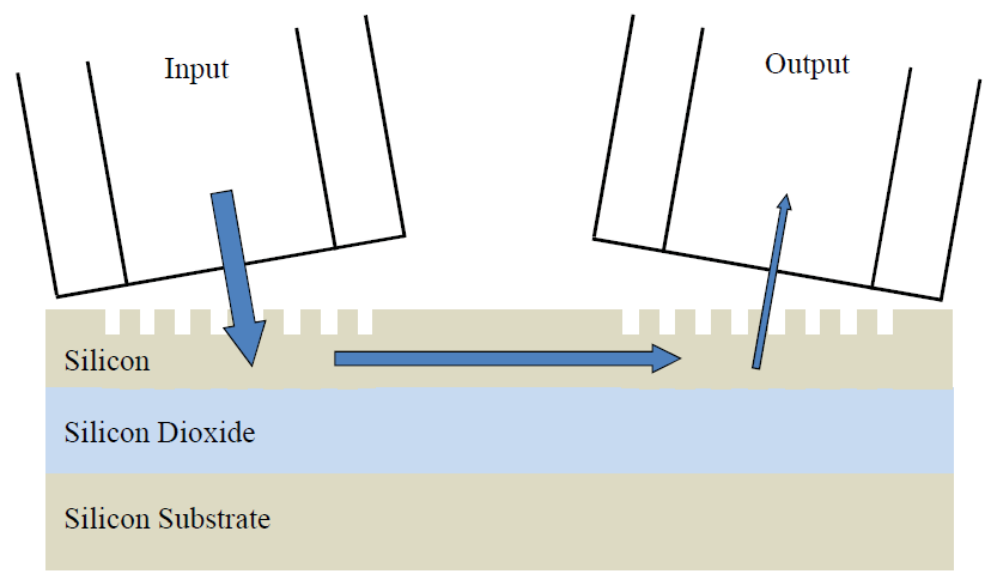

Figure 42: Grating couplers attached to the nano-waveguide [114].

\subsubsection{Mechanism of Grating Couplers}

Grating couplers were first proposed in 1970's with the aim of coupling free space laser light into glass films [114. The principle of grating couplers is based on a Bragg grating through which the laser light diffracts from free space into a dielectric waveguide. Similarly grating couplers can be used to diffract light from a waveguide into a free space detector. A simple example of this kind of grating coupler with waveguide is shown in Figure 42 . Figure 42 only shows the grating couplers and waveguide, the required additional tapers (cf. Figure 40) are suppressed for clarity. When the Bragg condition [115] is imposed on 
these grating couplers, we can describe optimal coupling as:

$$
n_{\text {eff }}=n_{\text {top }} \sin \left(\theta_{c}\right)+m \frac{\lambda}{\Lambda}
$$

Where,

$n_{\text {eff }}=$ Effective refractive index of grating

$n_{\text {top }}=$ Refractive index of the material on top of the grating

$\theta_{c}=$ Coupling angle measured perpendicular to the chip surface

$\lambda=$ Wavelength of incident light

$\Lambda=$ Grating period

This Equation 4.29 is only dependent on the modes of operation for gratings at a particular coupling angle. High coupling efficiencies between single mode fibers and the grating couplers are reported [116]. We already discussed in detail about the width and height of the waveguide and we understood that the width of the waveguide is much greater than either the waveguide height or the wavelength of light. This model (e.g. the width and height of waveguide) gives us the certainty of two dimensions which should be further reduced to one dimension. In this case the efficiency is

$$
\eta=\left|E\left(y, z=z_{\circ}\right) A e^{\frac{-\left(y-y_{\circ}\right)^{2}}{w_{\circ}^{2}}} e^{j y \frac{2 \pi}{\lambda} n_{t o p} \sin \left(\theta_{c}\right)} d y\right|^{2}
$$

Where,

$A=$ Constant describing the normalized Gaussian beam

$w_{\circ}=$ Beam waist

$y=$ Coordinate axis parallel to the waveguide axis

$z=$ Separation between the fiber and the top of grating

$z_{\circ}=$ Constant term

Equation 4.30 gives us the coupling efficiency for a grating to a fiber, but this equation does not tell the terms for the grating structure itself. The parameters consist of silicon top layer height, depth, grating period, buried oxide thickness and fill factor. All these 


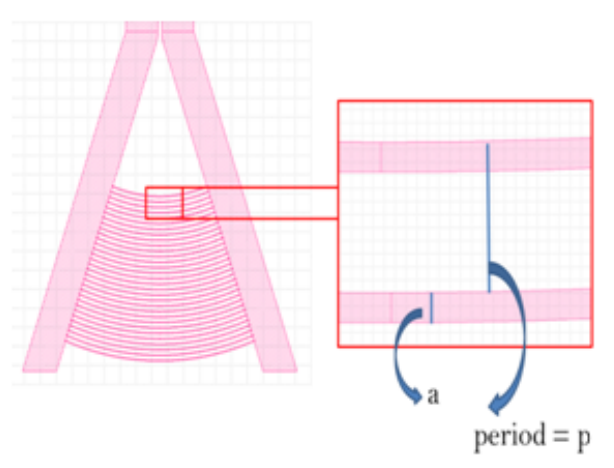

Fill factor $(\mathrm{ff})=\mathrm{a} / \mathrm{p} \cdot 100$

Figure 43: Grating coupler with calculating of fill factor " $f$ ".

structural parameters have a significant effect on the coupling efficiency, as is defined in $[117]$.

\section{Fill factor:}

The fill factor plays an important role for the efficiency. It is defined as ratio between the grating depth "a" to its period " $p$ ",

$$
\text { fill factor }(f f)=\frac{a}{p} .100
$$

The fill factor is shown in Figure 43.

\subsection{Fabrication of Silicon Waveguide Devices}

A Silicon-On-Insulator (SOI) wafer consists of a uniform $\mathrm{SiO}_{2}$ layer which is in the middle of a thick (hundreds of micrometer) silicon substrate and a thin surface layer of crystalline silicon. The thin surface layer of crystalline silicon forms the waveguide structure. After the waveguide, the grating couplers have to be fabricated on the silicon substrate, which are considerably more complicated then the waveguide because of the smaller dimensions involved and the high accuracy required. Both waveguide and grating couplers need different lithography and etching techniques that are specially suited for these kind of structures 118. 


\subsubsection{Nano-waveguide Fabrication}

There are two types of silicon etching in the fabrication of waveguides; dry and wet etching. Dry etching is the most suitable solution to meet the requirements of flexible process capability, reproducible production and high flexibility in fabrication of waveguides in SOI. For removing the upper layer in silicon dry etching, a chemically active plasma is used. This chemically active plasma is generated in a vacuum chamber to protect the sample from the external environment by an electromagnetic field. This electromagnetic field forms high-energy ions to improve on the silicon wafer surface and then react with it, which is known as reactive ion etching (RIE). The detail of the RIE and how it is used is found in [119]. The growth of $\mathrm{SiO}_{2}$ as a upper layer and the conversion of an asymmetrical waveguide to a symmetrical one is discussed in [120]. The general fabrication process of a SOI waveguide is shown in Figure 44.

\subsubsection{Grating Coupler Fabrication}

Here we briefly discuss three types of lithography techniques. For our Atom chip, we decided to use optical lithography for contact pads and wire but for the grating coupler we used e-beam lithography. It's because of the structures we need are too delicate for optical lithography. Therefore we used e-beam lithography.

\subsubsection{Optical Lithography}

The optical Lithography technique uses light image patterns on the photoresists (PR). Optical lithography creates a resist image on the silicon wafer. The later etching and lift off (ion implantation) processes are masked by the resist image at the areas dictated by the lithography mask. The patterns which are written on the silicon wafer are very small. This reduction of size is achieved by using a short wavelength. Instead of visible light, ultraviolet (UV) and deep ultraviolet (DUV) light sources are used (e.g. $<400 \mathrm{~nm}$ ). 


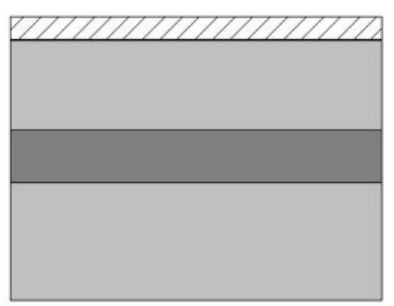

(a)

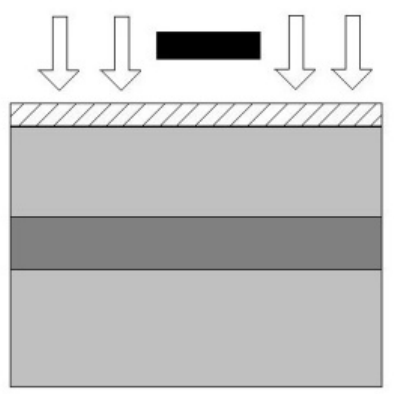

(b)

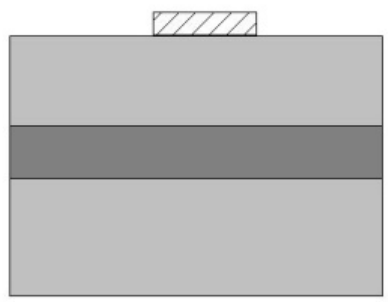

(c)

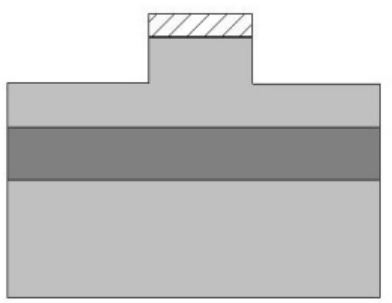

(d)

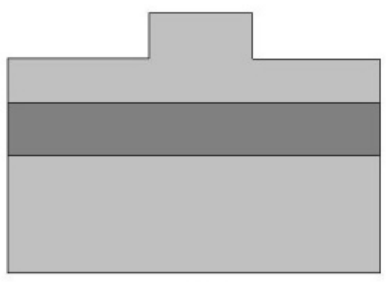

(e)

Figure 44: SOI waveguide fabrication process. (a) Previously cleaned and dried SOI wafer is coated with photoresist. (b) SOI wafer then aligned with the mask with sub micron precision and exposed to UV light, so the photosensitive elements of the resist are activated. This process is called photolithography. (c) Exposed parts are removed with the exposition of the wafer to a developing solution. (d) Silicon upper layer is etched to the desired thickness, (e) Remaining photoresist is removed [100]. 
Following are the process steps of optical lithography [120]:

- First we create a mask in optical lithography.

- Most of the time the mask should be fused-silica because fused silica has a low thermal expansion coefficient and higher transmission at short wavelengths (e.g. $200 \mathrm{~nm})$.

- Then mask resist coating is applied on mask substrate. Resist coating can be achieved by putting a small amount on liquid resist at the center of mask substrate followed by using a spinner which spins the mask substrate. Spin coating will result in a uniform coating of the liquid resist on the substrate.

- After spin coating, to fix the resist, the substrate will undergo baking.

- Next step will be to pattern the resist with the pattern generator. The patterns are generated by photographic means by electron beam lithography that can use a square or rectangular beam.

- After patterning the mask is developed and a metal coating (in our case gold) is applied.

- The metal coating fills the empty areas in the developed resist as well as on the top of the undeveloped resist.

- Finally the mask should be fully inspected and measured.

Above mentioned steps fully illustrate the general optical lithography method. Etch and lift of processes are shown in Figure 45 and 46 respectively. Further to that, photoresists are divided into three types:

\section{Positive Photoresist (PR):}

Positive PR pattern is the same as the mask but on exposure to light, light degrades the polymers resulting in the PR being more soluble in developers. The PR can be removed by inexpensive solvents such as acetone. We used positive PR in our atom chip fabrication. 


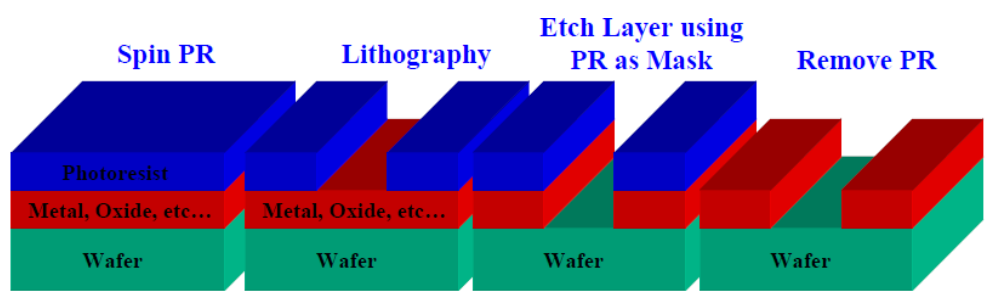

Figure 45: Etching Processes: open windows in oxides for diffusion, masks for ion implantation, etching, metal contact 120 .

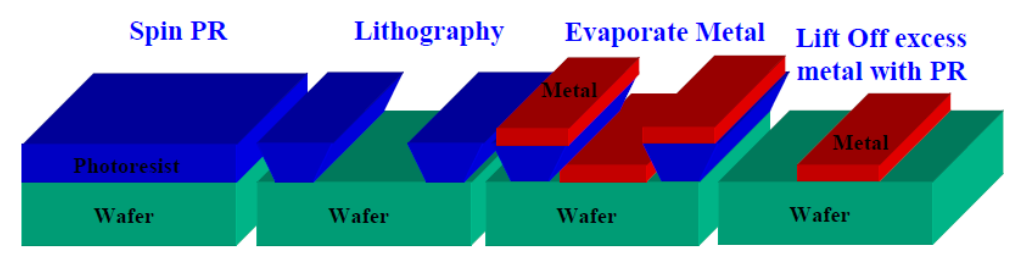

Figure 46: Lift off Processes: Metalization 120.

\section{Negative Photoresist (PR):}

Negative PR pattern is the inverse of the mask. On exposure to light, light polymerizes the rubbers in the $\mathrm{PR}$, to strengthen its resistance to dissolution in the developer. Resist has to be removed in special stripping chemicals.

\section{Combination:}

Combination PR is not very significant, same PR can be used for both negative and positive pattern transfer. Combination PR can be removed in inexpensive solvents [120].

\subsubsection{Electron Beam Lithography}

In this technique, a focused electron beam scans the surface of the photoresist. The pattern are written directly onto the photoresist and no mask is necessary. The spot size of the beam is very small, down to $5 \mathrm{~nm}$, therefore this technique is highly flexible. Nevertheless this technique has the disadvantage of being quite slow and not suitable for mass production $[120]$. 


\subsubsection{Interference Lithography}

Two coherent beams coming from a laser are combined to form a periodic intensity pattern in this technique. The angle between beam 1 and beam 2 determines the grating period. In this technique we can define about $250 \mathrm{~nm}$ of grating periods [120].

\subsubsection{Dry Etching}

In this technique we used dry etching process such as RIE (this technique also ideal for waveguides). Different etch depths will control the etch depth, error in this depth can be under $5 \mathrm{~nm}$ (this will need a well controlled fabrication environment) [120]. 


\section{Atom Chip: Design, Simulation and Fabrication}

\subsection{Introduction}

In this chapter we will discuss the atom chip to be used in the experiment foreseen in the present thesis in detail. We will explain how we design, simulate and fabricate the chip. Later on we will discuss how to bring the cold atoms near the surface of the chip. With the MOT we can neither achieve high densities of our Rb cloud nor can we bring the MOT really close to the surface of the chip. So we need to transfer the atoms into a magnetic trap that is easier manipulated than a MOT. This magnetic trap will be formed by a wire the shape resembling a ' $z$ ' on the chip, henceforth called z-wire, plus the external bias field coils. We design the chip such that a current will pass through its wires and make a magnetic field to be almost zero at the center of the chip, where cold atoms will come near to the surface of the chip. Also at the center we fabricate grating couplers with attached nano-waveguides; the cold Rb atoms will come near the surfaces of these nano-waveguides. Each waveguide will be connected to two couplers. One serves to couple a focused laser beam into the waveguide. The second coupler is to re-emit the light after transmission trough the waveguide into free space. This light will contain information on possible atom-light interactions and will be refocused onto a high-sensitivity photodetector.

This atom chip will be employed in the setup that we already introduced in Chapter 3 . For that reason we design this chip in a manner to be easily attached to the apparatus and also to be easily detached as well. This way we can use different chips according to our requirements.

\subsection{Design of the Atom Chip}

The design of the chip is based on the calculations and simulations performed within the present work to accommodate both the optical requirements for experiments using photonic structures and for the creation of a cloud of cold Rb atoms in close vicinity 


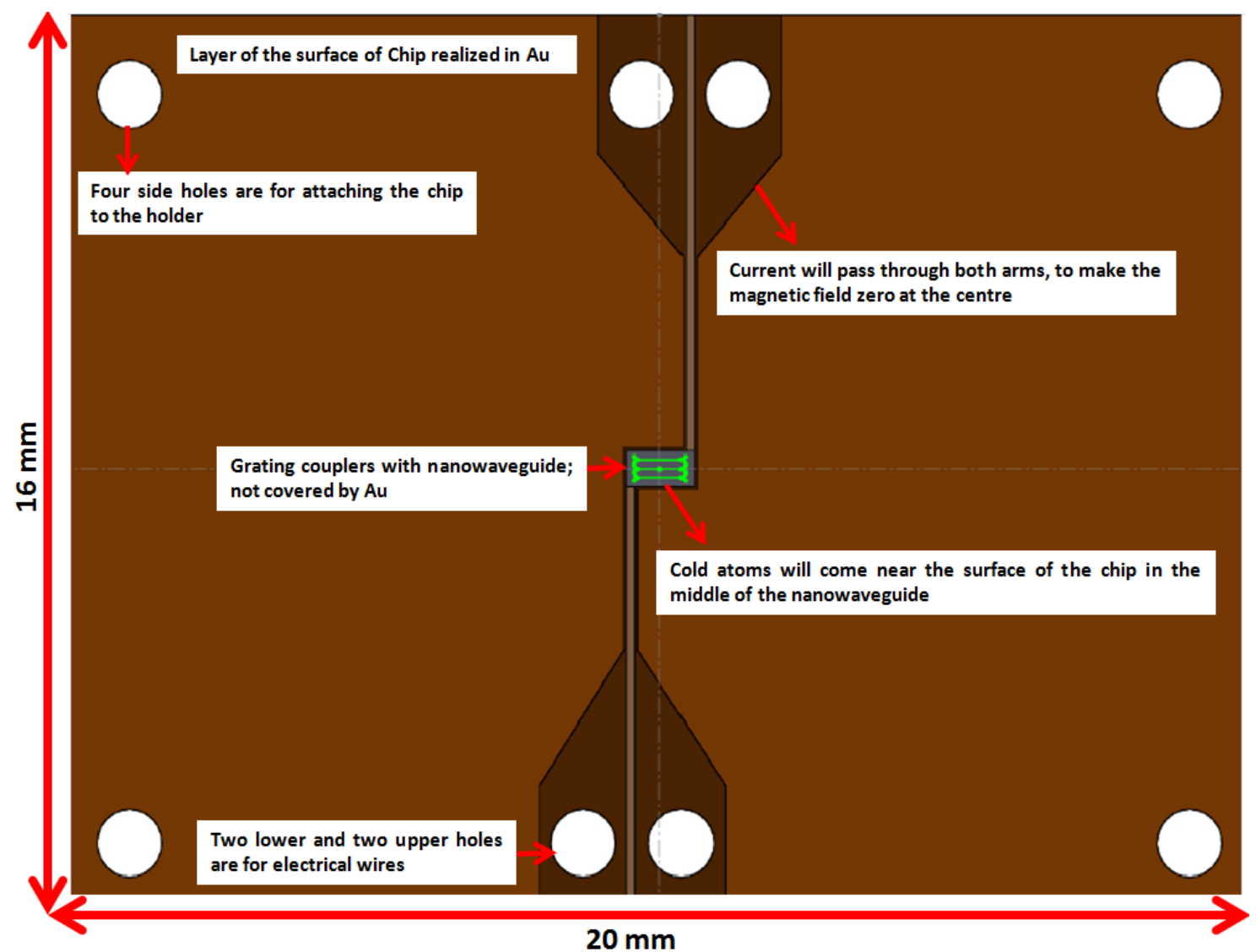

Figure 47: Atom Chip that used in Cold atom experiment.

(discussed in Chapter 3). The chip will be at the center of the glass cell and will be attached to the metallic rod. This rod will bring and position the chip at the center of the glass cell. At the center of the chip we introduced our grating couplers with nanowaveguide. Most of the surface of chip will be fabricated with a gold (Au) layer except of the central area where the grating couplers and waveguides will be located. The Au layer on the surface of the chip is fabricated to provide the required reflectivity for the realization of the mirror MOT, where the chip serves as mirror surface for two of the four MOT beams.

\subsubsection{Parameters of the Atom Chip}

The Atom chip is $16 \times 20 \mathrm{~mm}$ in size. This size is determined by the dimensions of the UHV glass cell and the inner diameters of the connecting vacuum components that lead 
to the load-lock system. Figure 47 shows the size of the Atom chip with red arrows. In Figure 47 there are four holes at the corners of the chip. Each hole has a diameter of $1.50 \mathrm{~mm}$. These holes attach the chip to the holder with the help of screws. There are four other holes at the upper and lower center of the chip, these holes are also of $1.50 \mathrm{~mm}$ diameter. Those are for the electrical current, and external wires will be attached to these holes (contact pads) to allow the current to flow in the chip. The distance between these two arms or contact pads is $0.20 \mathrm{~mm}$. There is also a small distance/separation of $10 \mu \mathrm{m}$ between the arms and chip surface. We make this separation to electrically isolate the wires from the rest of the chip. The couplers and waveguides are fabricated at the center. This area is $1 \times 1 \mathrm{~mm}$ in size and it is not covered with $\mathrm{Au}$. We left this area without $\mathrm{Au}$ so that grating couplers and waveguides will not be fabricated on a metal surface. Central area is also visible in Figure 47.

\subsection{Simulation of Atom Chip}

Simulation is an important part because we have to calculate exactly how much current we should run in the wires of the chip so that we can bring the cold atom cloud near to the surface of the chip. We also calculate the magnetic field and difference in corresponding energies (trap depth). We did our chip simulation in Wolfram Mathematica programming language. We designed two z-shape electrical wires that are parallel to each other. We gave the dimensions of these wires and run a current in it. Several simulations with different dimensions of the wires to get optimal results were performed.

Figure 48 shows the two wires in which the electrical current will pass through. When we pass a certain current in both arms we can get the magnetic field almost zero at the center of the arms and we can trap the cold atoms. Figure 49 shows the simulation results in which we give the current $1.5 \mathrm{~A}$ to both arms with thickness $0.4 \mu \mathrm{m}$, width $300 \mu \mathrm{m}$, arms length $7.5 \mathrm{~mm}$, middle line length $1 \mathrm{~mm}$. We get the trap center $90 \mu \mathrm{m}$ from the surface of chip. Figure 49 shows three plots for $\mathrm{x}$-axis, $\mathrm{y}$-axis and z-axis respectively, we 


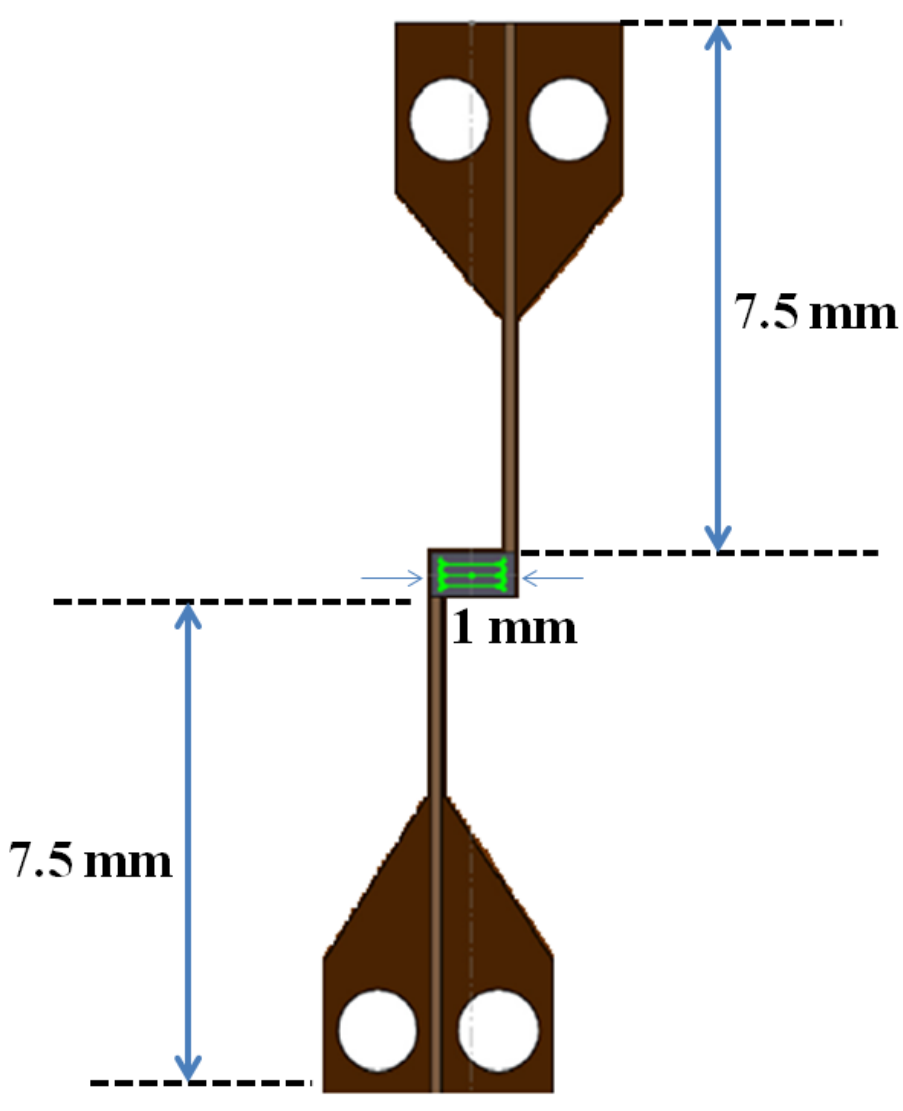

Figure 48: z-shaped double wire structure with grating couplers and nano-waveguide at the center, holes are going to used for electrical pads. 

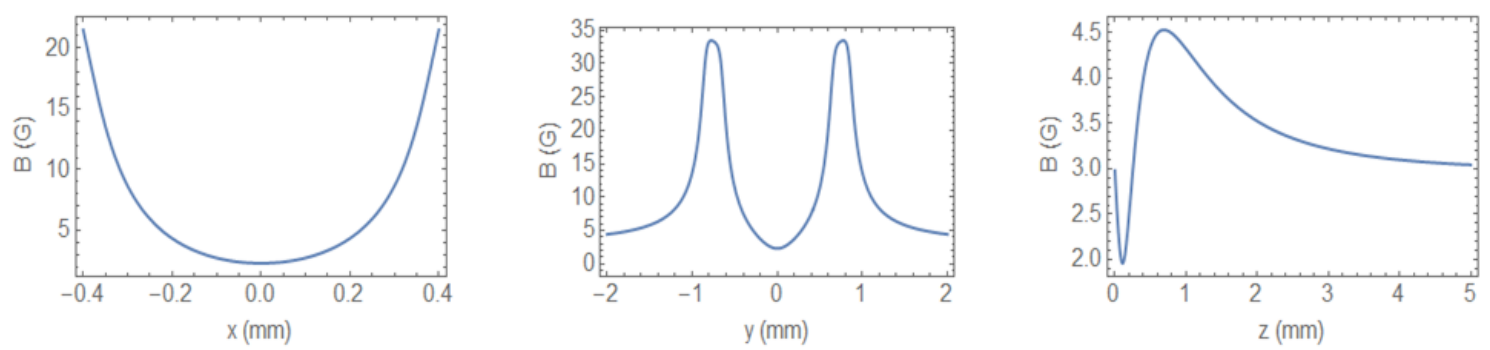

Figure 49: Simulation result for double z-wire structure with thickness $0.0004 \mathrm{~mm}$, width 0.3 $\mathrm{mm}$, arms length $7.5 \mathrm{~mm}$, middle line length (zlength) $1 \mathrm{~mm}$, trap center from the surface of chip $90 \mu \mathrm{m}$ and current running in wire is $1.5 \mathrm{~A}$.

can clearly see that for $\mathrm{x}$-axis and $\mathrm{y}$-axis graphs the magnetic field is small at the center but for z-axis.

In case of increased/decreased current, width and length of wires, we are getting the trap further away from the surface of chip. Therefore we chose above parameters and the results that are shown in Figure 49.

We calculate the final energy by using the interaction Hamiltonian equation from [40]

$$
H_{B}=\mu_{B} g_{F} m_{F} B_{z}
$$

Where,

$g_{F}=$ Hyperfine Landé g-factor

$B_{z}=$ Magnetic field in z-direction

$m_{F}=$ Hyperfine splitting (quantum number)

$\mu_{B}=$ Bohr magneton $\left(9.27400915 \times 10^{-24} \mathrm{~J} / \mathrm{T}\right)$

Where the hyperfine Landé g-factor is given by

$$
g_{F}=g_{J} \frac{F(F+1)-I(I+1)+J(J+1)}{2 F(F+1)}+g_{I} \frac{F(F+1)+I(I+1)-J(J+1)}{2 F(F+1)}
$$

Now, if we neglect the nuclear term $g_{I}$, which is much smaller then $g_{J}$, we get

$$
g_{F} \cong g_{J} \frac{F(F+1)-I(I+1)+J(J+1)}{2 F(F+1)}
$$


For weak magnetic fields, the interaction Hamiltonian $H_{B}$ perturbs the zero field, eigenstates of $H_{h}$. To lowest order, the levels split linearly.

$$
\Delta E_{\left|F m_{f}\right\rangle}=\mu_{B} g_{F} m_{z} B_{z}
$$

Figure 50 shows that we are using the D2 line, $5^{2} S_{\frac{1}{2}} \rightarrow 5^{2} P_{\frac{3}{2}}$, and it is in our case $F=2, m_{F}=+2$, for the ${ }^{2} S_{\frac{1}{2}}$ ground state.

We know

$\mu_{B}=9.27400915 \times 10^{-24} \mathrm{~J} / \mathrm{T}$

$g_{F}=\frac{1}{2}$

$m_{F}=+2$

$B_{z}=3 G$ (Bias field in z-direction)

By putting all these values in Equation 5.4, we arrive at

$$
\begin{gathered}
\Delta E_{\left|F m_{f}\right\rangle}=\left(9.27400915 \times 10^{-24} J / T\right)\left(\frac{1}{2}\right)(2)(3.5) \\
\Delta E_{\left|F m_{f}\right\rangle}=2.78220275 \times 10^{-27} \mathrm{~J}
\end{gathered}
$$

To convert in Kelvin we have

$$
\begin{gathered}
\Delta E_{\left|F m_{f}\right\rangle}=\frac{2.78220275 \times 10^{-27} J}{1.3806488 \times 10^{-23} J K^{-1}} k_{B} \\
\Delta E_{\left|F m_{f}\right\rangle}=202 \mu \mathrm{K} \mathrm{k} \mathrm{k}_{\mathrm{B}}
\end{gathered}
$$

We have the final results:

Magnetic field at bottom of trap $=2 \mathrm{G}$ with $90 \mu \mathrm{m}$ away

Highest magnetic field when going away from chip $=5 \mathrm{G}$ with at least $500 \mu \mathrm{m}$ away Differences in corresponding energies $=3 \mathrm{G}$ 


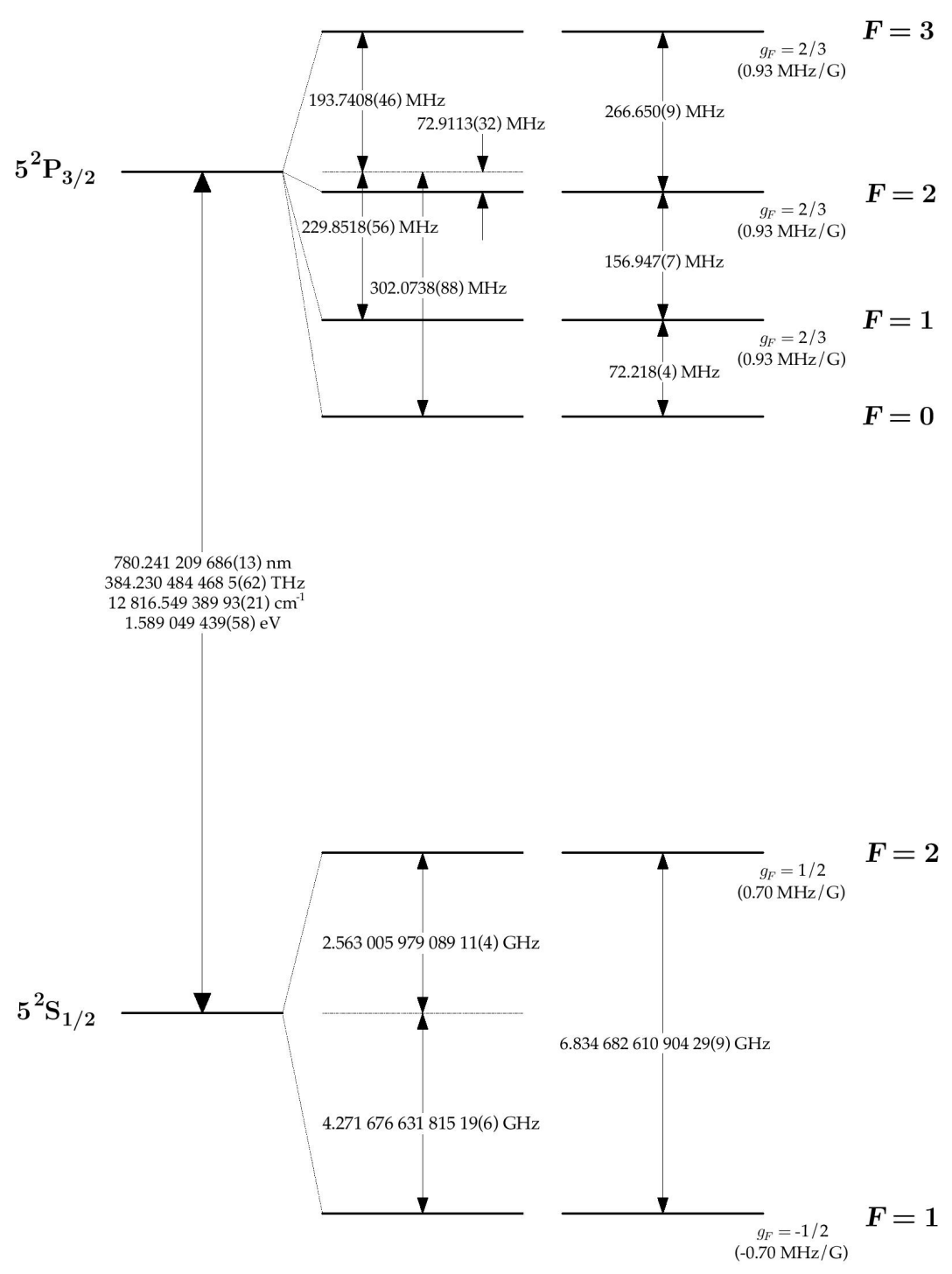

Figure 50: Hyperfine transition structure of ${ }^{87} R b D_{2}$ line, with frequency splitting between hyperfine energy levels, approximate Landé $g_{F}$ factor and Zeeman splittings 40 . 


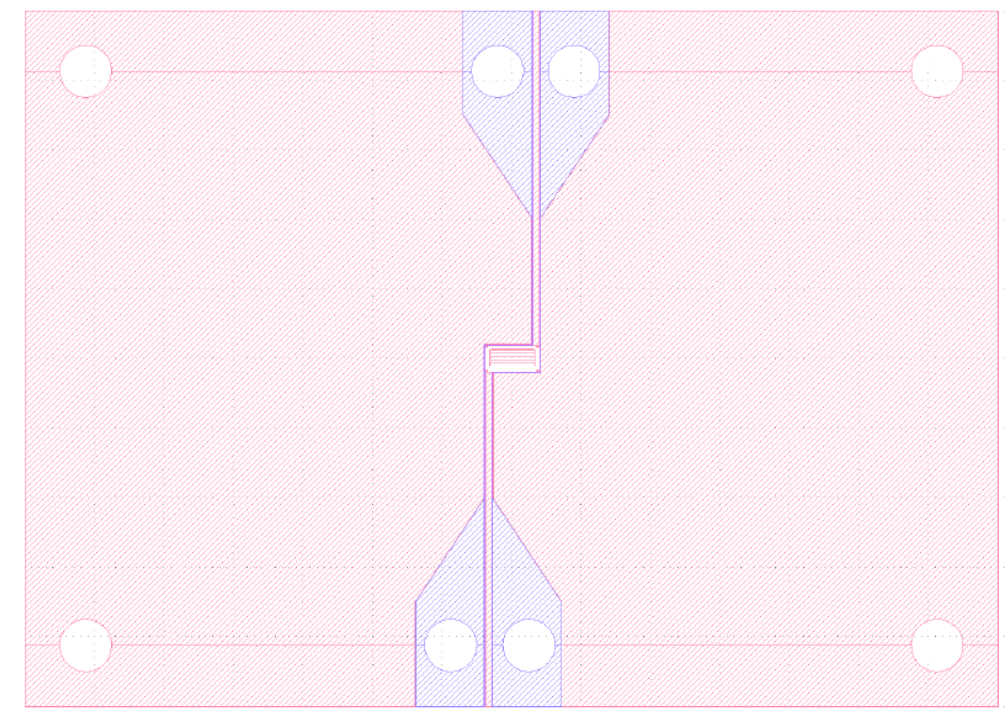

Figure 51: Design made in Python programming language for Atom chip that will later used in e-beam lithography as a mask.

\subsection{Fabrication of Atom Chip}

For fabrication first we have to create a mask. In other words, we have to make a design in a so called gds file because our e-beam lithography machine only accepts gds files. A gds file can be created in different programming languages. In our case we used the Python programming language to realize the design of the chip. The design is shown in Figure 51

The next step is to fabricate the chip. We used the optical lithography method that we already discussed in detail in Chapter 4 . Figure 52 shows the pattern of the fabrication and the parameters we used for the layers. The upper most layer of silicon nitride ( $\mathrm{SiN})$ is $200 \mathrm{~nm}$. From this layer our grating couplers and waveguides will be fabricated. Except for the central area in SiN the rest of the chip is covered by a gold $(\mathrm{Au})$ layer. The thickness of the Au layer is $100 \mathrm{~nm}-200 \mathrm{~nm}$. There is a silicon dioxide $\left(\mathrm{SiO}_{2}\right)$ layer of $2 \mu \mathrm{m}$ beneath the $\mathrm{Au}$ layer. This $\mathrm{SiO}_{2}$ makes the reflection more confined and stops the leakage of light in grating couplers and waveguides. All upper layers are fabricated in industrial available silicon wafer of $5 \mathrm{~mm}$ thickness. We fabricate a base of a chip with copper $(\mathrm{Cu})$ of $1 \mathrm{~mm}$. This layer is due to the high thermal conductivity of copper. It can help to remove heat from the hot wires on the surface of the chip. This copper layer 


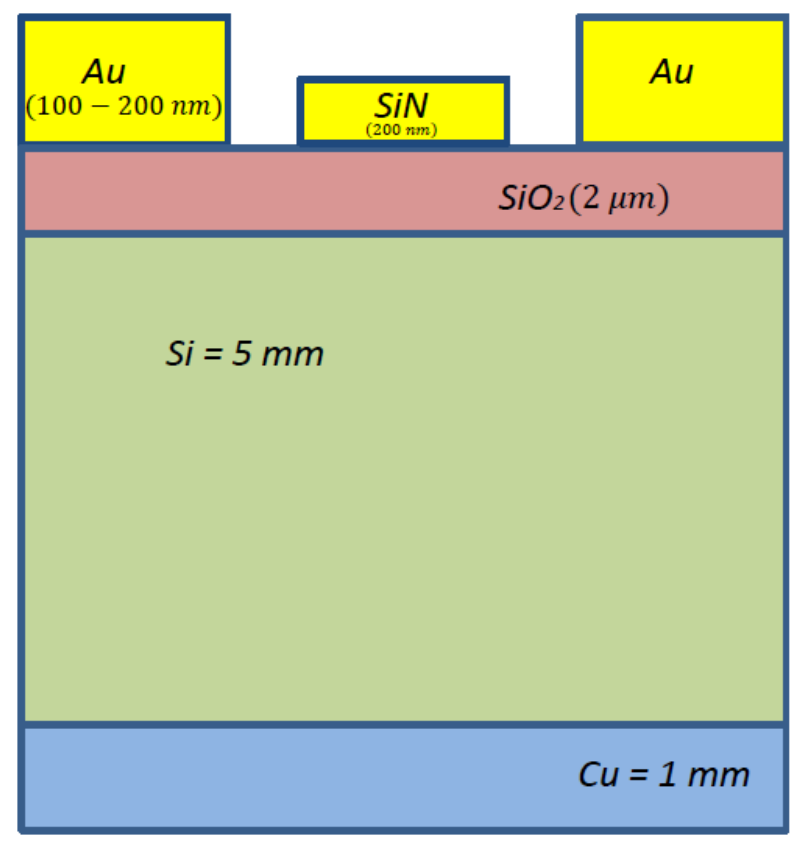

Figure 52: Silicon wafer $(\mathrm{Si})$; at top gold a $(\mathrm{Au})$ layer of $100-200 \mathrm{~nm}$ is fabricated, between $\mathrm{Si}$ and $\mathrm{Au}$ a layer $\mathrm{SiO}_{2}$ layer is fabricated for guidance of $\mathrm{Au}$ layer. At bottom of $\mathrm{Si}$ wafer a copper $(\mathrm{Cu})$ layer of $1 \mathrm{~mm}$ is fabricated.

could also serve to establish thermal contact to the mounting base of the movable rod.

For simplicity and for a first test, we first fabricate and design the grating couplers and waveguides on a chip without a layer of $\mathrm{Au}$. We want to see which parameters for the grating couplers and waveguides are giving us best transmission properties at a wavelength of $780 \mathrm{~nm}$. These grating couplers and waveguides are also designed in Python programming language. At first we designed several grating couplers on a chip that is shown in Figure 53 .

We increase the filling factor from top to bottom and increase the design central wavelength from left to right as shown in Figure 53. The fabricated grating couplers with waveguide and their SEM (Scanning Electron Microscopy) image is shown in Figure 55 . Figure 56 and Figure 57 show the structures in increasing detail. For selecting the best grating coupler we used the experimental set up as described in [121], and shown in Figure 54. We used a diode laser, that will simple first pass through the polarization controller. After passing through polarizing controller the laser will go to the stage where our chip (fabricated with couplers) is placed. The laser will pass to the grating coupler and through 


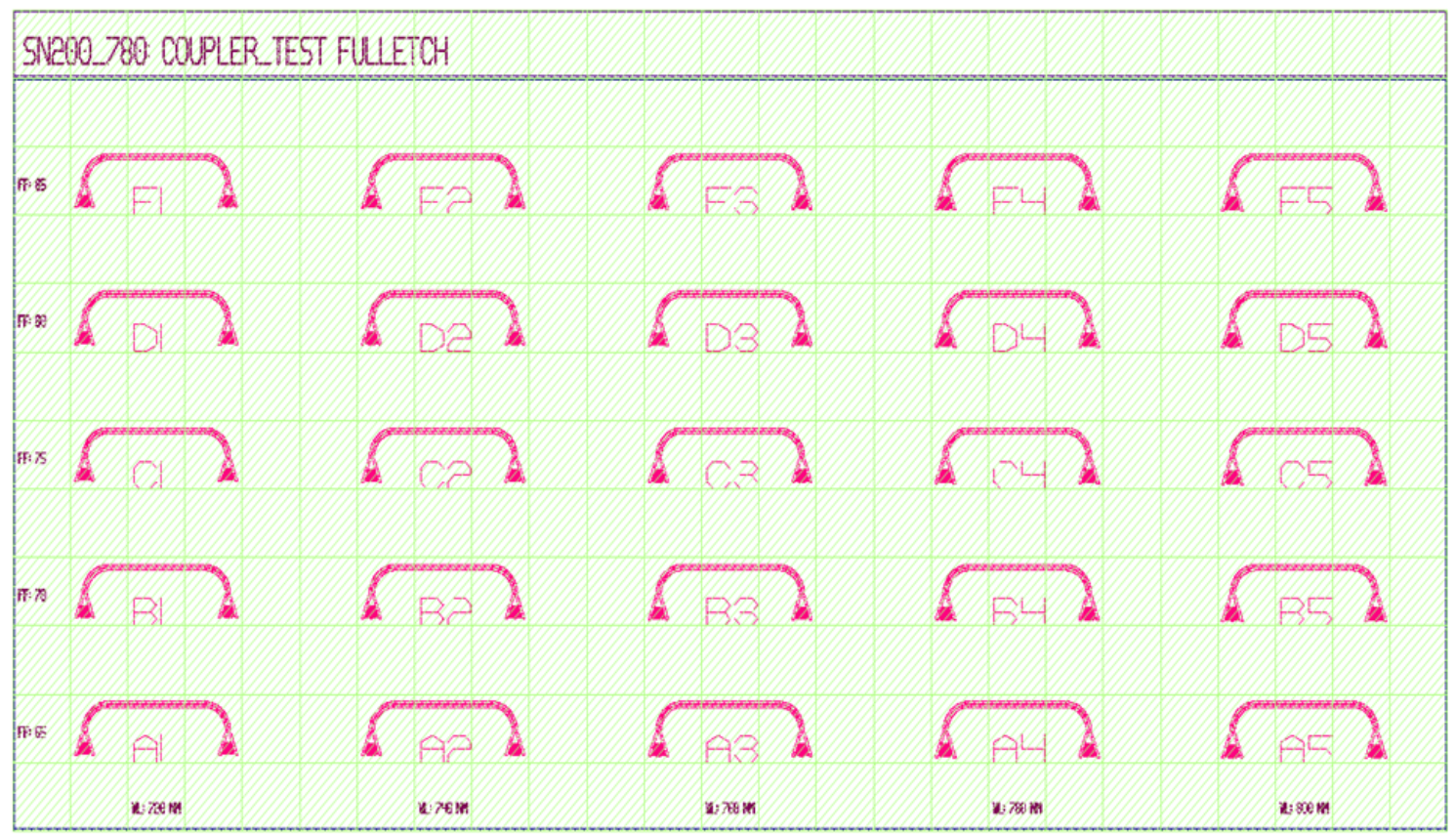

Figure 53: Mask generated in Python for grating couplers and nano-waveguides. The coupler fill factor (ff) increases from 65 (bottom) to 85 (top). In horizontal direction the grating period expressed in term of an expected design central wavelength increases from $720 \mathrm{~nm}$ (left) to $800 \mathrm{~nm}$ (right).

the waveguide, and will come out again through the opposite grating coupler. On output of the grating coupler we have photo-detector as shown in Figure 54. We examined each couplers transmission value one by one. We get the best transmission peak for the coupler that is shown in Figure 56. The transmission values for the best two grating couplers are shown in Figure 58 .

We get these results from the grating coupler D5 and D1 that are given in Figure 55. We used our experiment set-up (shown in Figure 54) in KIT to gain this result. In Figure 58 we clearly see that D5 grating coupler giving us the best transmission value. Best transmission is achieved for D5 coupler. From Figure 53 we can see that D5 coupler has a ff (fill factor) value 80 and wavelength $800 \mathrm{~nm}$. D5 coupler is going to be used in our Atom Chip. 


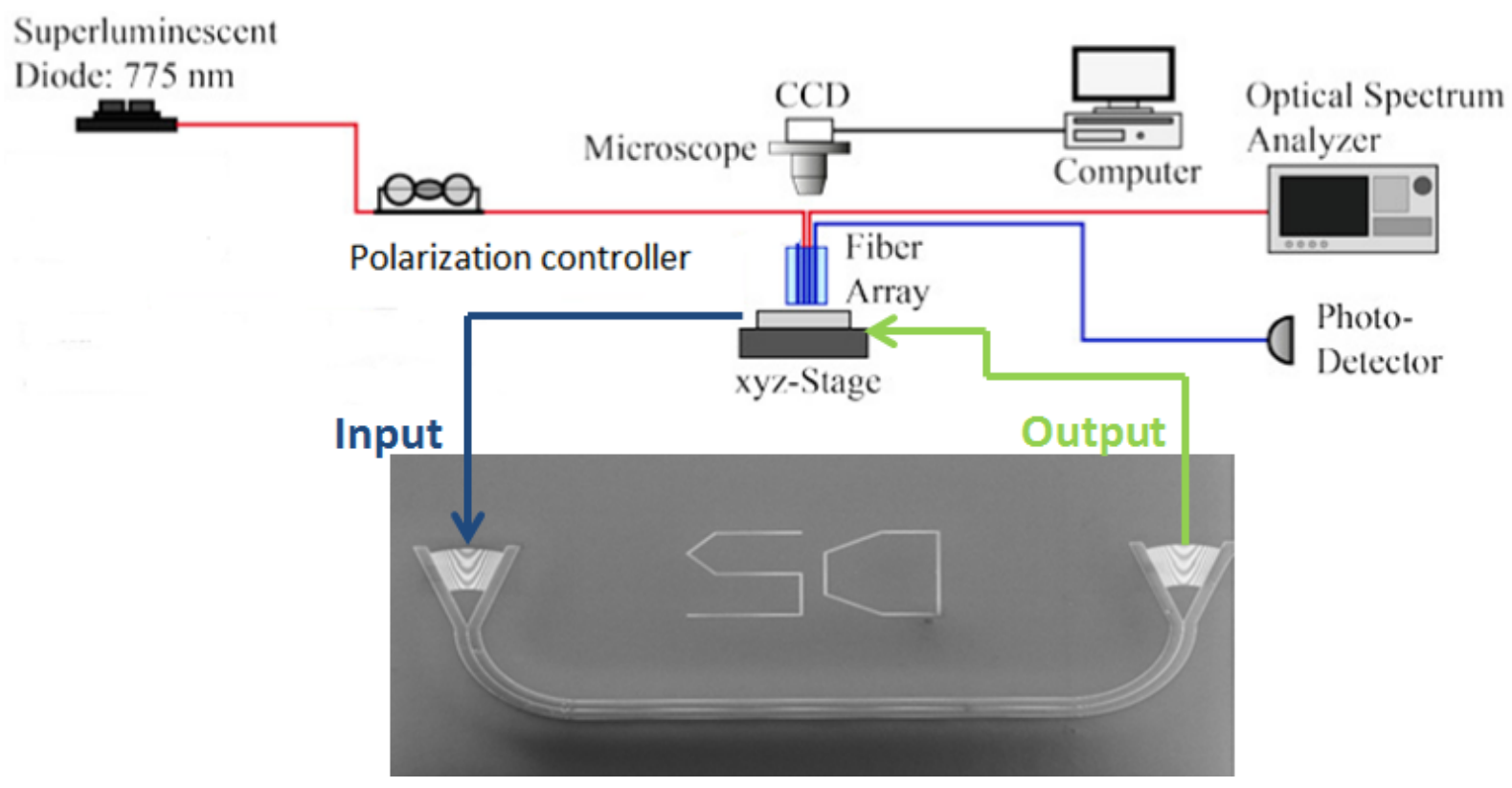

Figure 54: Experimental set-up in KIT for measuring the grating couplers Q-factor (transmission values) [121.

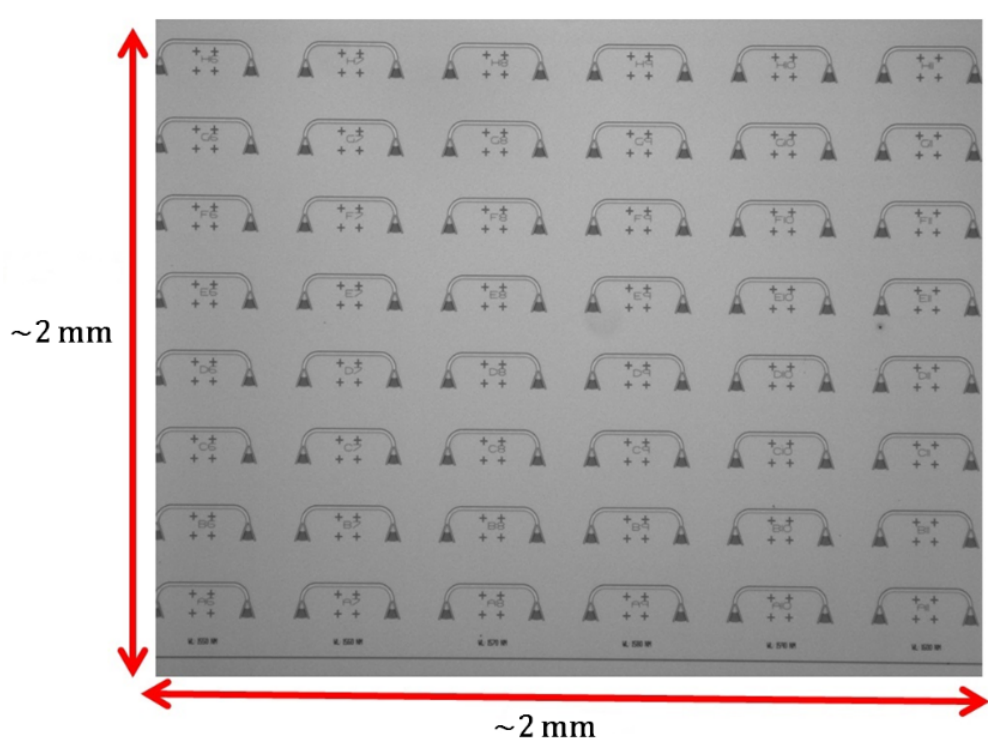

Figure 55: Scanning electron microscopy (SEM) image of grating couplers with nanowaveguides that are fabricated on Si chip. 


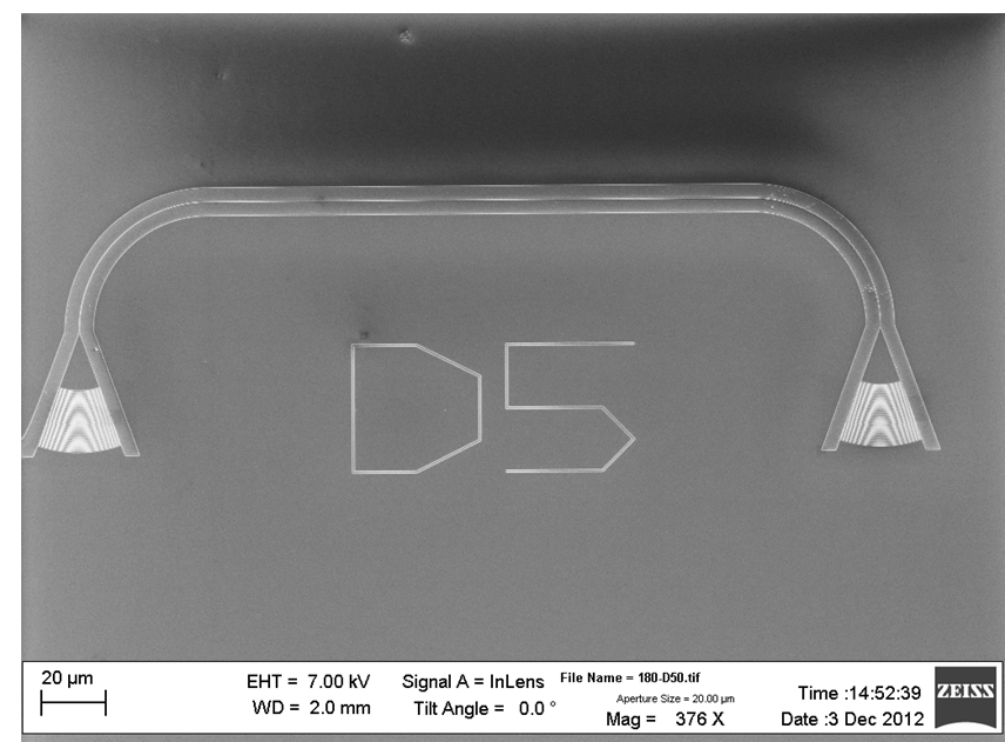

Figure 56: Detailed SEM image of the nano-waveguide with attached grating coupler structures that showed the best transmission at the required wavelength of $780 \mathrm{~nm}$.

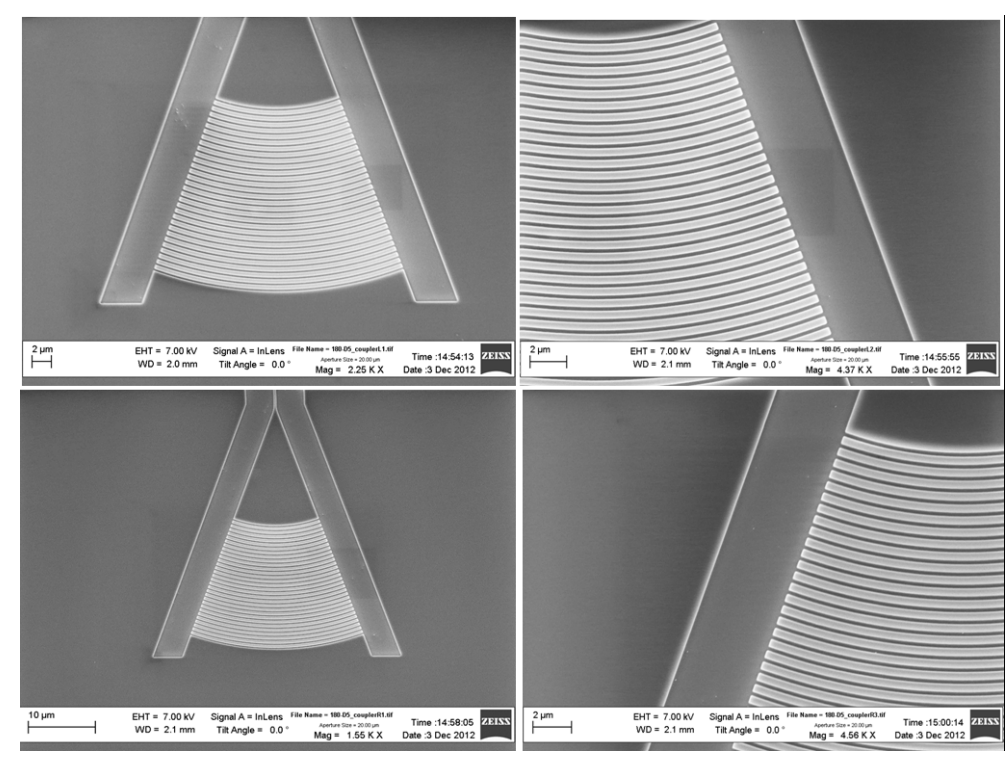

Figure 57: 2 and $10 \mu \mathrm{m}$ SEM images of grating couplers. 


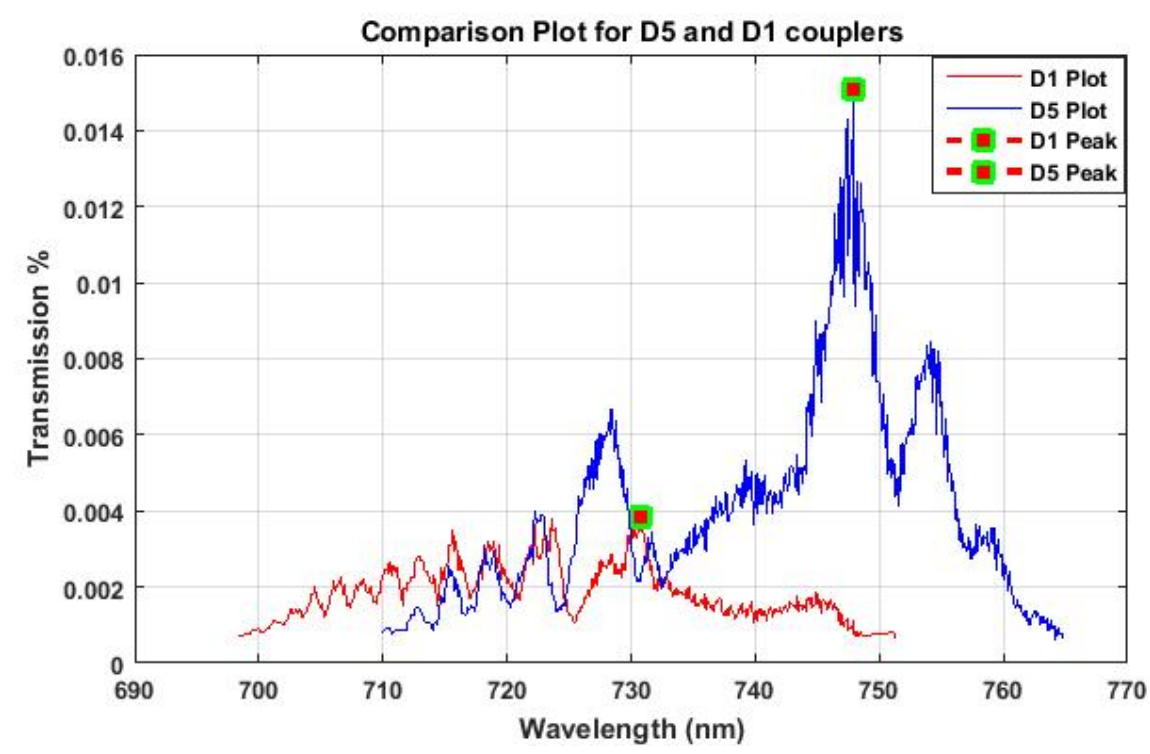

Figure 58: Comparison plot between D1 and D5 coupler, we can clearly see that D5 coupler giving us a best transmission value, Figure 56 showing the D5 coupler, (Plot generated in MATLAB). 


\section{Conclusion and Future Work}

\subsection{Experiment status}

For the cold atom experiment in LENS institute, we currently have ready to use our UHV cell, linear shift mechanism, Rb dispenser, metallic bars that will used in to support the set up and various optics component. Next we planned to build the BIAS and antiHelmholtz coils in laboratory, the design and simulation of these coils are already discussed in Chapter 3. We were also planning to make the necessary power supplies for these coils in laboratory. Ion pump, gate valve and metallic rod for supporting chip are in principle ready to be ordered.

For the Atom chip in KIT university, we already simulated, and designed the chip, and we did test a few fabrication techniques as discussed in Chapter 5. In the future we are planning to fabricate the Atom Chip with the parameters that we already simulated. Our next step is to bring the Atom Chip in LENS laboratory and to place in our cold atom experiment. Our first goal is to make the cold atom experiment run in the presence of Atom Chip. We are optimistic that this new experiment will give us a unique results. On the basis of these results we want to study possible atom-light interactions in-depth.

\subsection{Application}

The current project has significant potential to provide insight into the tailored control of light fields and photonic memories. It is as such of basic interest for future information technology applications where the fast and minute control of quantum light fields will be the basic building block of state-of-the art communication protocols. Possible applications range from single photon sources for cryptographic and information processing applications to repeater and storage devices for future all optical networks to high precision sensors for light and matter fields.

Our approach takes the established industry graded silicon technology and combines it 
with leading edge atomic physics techniques in order to explore the range of possible commercially interesting applications. The novel replaceable chip design will allow for rapid adoptions of the experiment to new requirements and guarantees that industrially interesting settings can be maintained also in future.

\subsection{Conclusion}

Our experimental efforts will provide the ideal platform to the study of matter-light interactions. First studies are to concern the influence of ${ }^{87} R b$ atoms on the $\mathrm{Q}$ factors of resonating structures. Applications in the field of optical switches and high-precision sensors are perceivable. Based on these results new Chip designs with enhanced possibilities will be developed and, due to the modular load-lock system, rapidly put to study.

Cold and ultra-cold atoms have made a number of important contributions to improve our understanding of fundamental physics, quantum information, quantum interferometry and many other related fields. Furthermore light-matter interaction is a basic tool in physics research and gaining importance in applications day by day. Modern production techniques such as electron beam lithography nowadays already offer the possibility to create highly functional photonic structures within Silicon wafer substrates that give us high control on photonic fields. We combine the available techniques to precisely control both, light fields and cold atoms, in order to study the new phenomena when the two of them step into close interaction.

\subsection{Future Work}

The uniqueness of the project lies on changing the Atom Chip frequently. This means that one can easily change the active structures on the Atom Chip. Due to this, we are able to rather easily extend our studies to investigations of the behavior of plasmonic metamaterials and other interesting structures, such as carbon nano-tubes, in close contact 
to atomic samples. Finally, while at present the experiment only aims to realizing a cold atoms cloud, the setup is versatile enough to enhance the chip functionality by embedding further electronic circuits that allows us to attain degenerate atomic samples, Bose-Einstein condensates. In this realm even stronger mutual influences between light fields and matter waves are to be expected. 


\section{References}

[1] A. Ashkin, "Atomic-beam deflection by resonance-radiation pressure," Phys. Rev. Letts, vol. 25, no. 19, pp. 1321-1324, 1970.

[2] T. W. Hänsch and A. L. Schawlow, "Cooling of gasses by laser radiation," Optics Communication, vol. 13, no. 1, pp. 68-69, 1975.

[3] D. G. Wineland, R. E. Drullinger, and F. L. Walls, "Radiation-pressure cooling of bound resonant absorbers," Phys. Rev. Letts, vol. 40, no. 25, pp. 1639-1642, 1978.

[4] W. Neuhauser, M. Hohenstatt, H. Dehmelt, and P. Toschek, "Optical-sideband cooling of visible atom cloud confined in parabolic well," Phys. Rev. Letts, vol. 41, no. 4, pp. 233-236, 1978.

[5] D. J. Wineland and H. Dehmelt, "Proposed $10^{14}$ laser fluorescence spectroscopy on ti $i^{+}$mono-ion oscillator iii," Bull. Am. Phys. Soc, vol. 20, p. 637, 1975.

[6] W. D. Phillips and H. J. Metcalf, "Cooling and trapping atoms," Scientific American, INC, pp. 50-56, 31987.

[7] C. Cohen-Tannoudji and W. D. Phillips, "New mechanisms for laser cooling," Physics Today, p. 33, 101990.

[8] S. Chu, "Laser trapping of neutral particles," Scientific American, INC, p. 71, 2 1992.

[9] D. A. Jones, "Electrical engineering: the backbone of society," Proceedings of the IEE: Science, Measurement and Technology, vol. 138, no. 1, pp. 1-10, 1991.

[10] C. K. Alexander and M. N. O. Sadiku, Fundamentals of Electric Circuits. 5, McGraw-Hill, 2013.

[11] K. Erickson, Laser. http://spaceplace.nasa.gov/laser/en/l. 
[12] A. L. Schawlow and C. H. Townes, "Infrared and optical masers," Phys. Rev. Letts., vol. 112, no. 6, pp. 1940-1950, 1958.

[13] J. M. Kahn and J. R. Barry, "Wireless infrared communications," Proceedings of the IEEE, vol. 85, no. 2, pp. 265-298, 1997.

[14] I. F. Akyildiz, W. Su, Y. Sankarasubramaniam, and E. Cayirci, "Wireless sensor networks: a survey," Computer Networks, vol. 38, no. 4, pp. 393-422, 2002.

[15] K. V. Acoleyen, W. Bogaerts, J. Jagerska, N. L. Thomas, R. Houdre, and R. Baets, "Off-chip beam steering with a one-dimensional optical phased array on silicon-oninsulator," Optics Letters, vol. 34, no. 9, pp. 1477-1479, 2009.

[16] A. L. Schawlow, "Spectroscopy in a new light," Rev of Mod Phys., vol. 54, no. 3, pp. 697-707, 1982.

[17] A. A. Michelson, Recent advances in spectroscopy, 1907. http://www.nobelprize. org/nobel_prizes/physics/laureates/1907/michelson-lecture.pdf.

[18] A. B. Aronst and M. B. Peppard, "Einstien's proposal of the photon concept a translation of the annalen der physik paper 1905," American journal of physics, vol. 33, no. 5, pp. 367-374, 1965.

[19] R. A. Serway, C. J. Moses, and C. A. Moyer, Modern Physics. 3, David Harris, 2005.

[20] A. M. Ellis, M. Feher, and T. G. Wright, Electronic and Photoelectron Spectroscopy. 1, Cambridge University Press, 2005. physics.

[21] G. J. Aubrecht, "The nuclear science wall chart," The Physics Teacher, vol. 33, no. 35, p. 544, 1997.

[22] M. A. Farrukh, Advanced Aspects of Spectroscopy. 1, InTech, 2012.

[23] G. Lockemann and R. Oesper, "Bunsen's transfer from cassel to marburg," J. Chem. Educ., vol. 32, no. 9, pp. 456-460, 1955. 
[24] Weeks and M. Elvira, "The discovery of the elements. xlll. some spectroscopis discoveries," J. Chem. Educ., vol. 9, no. 8, pp. 1413-1434, 1932.

[25] Ritter and K. Stephen, "C and en: it's elemental: The periodic table-cesium," American Chemical Society, 2003.

[26] C. J. Eidson, "Gps," Measurement, control, and communication using IEEE 1588, p. $32,2006$.

[27] T. King and D. Newson, "Rubidium and crystal oscillators," Data network engineering, p. 300, 1999.

[28] L. Marton, Advances in electronics and electron physicsl, vol. 44. ELSEVIER, 1 1977.

[29] N. F. Ramsey, "History of atomic clocks," Journal of research of the national bureau of standard, vol. 88, no. 5, pp. 301-320, 1983.

[30] P. V. Tryon and R. K. Jones, "Estimation of parameters in models for cesium beam atomic clocks," Journal of research of the national bureau of standard, vol. 88, no. 1, pp. 3-16, 1983.

[31] A. C. Melissinos and J. Napolitano, Experiment in Modern Physics. Elsevier Science (USA), 2003.

[32] K. Gottfried and T. M. Yan, Quantum Mechanics: Fundamentals. 2, Springer, 2004.

[33] B. H. Bransden and C. J. Joachain, Physics of Atoms and Molecules. Prentice Hall, 2 ed., 2003.

[34] V. Zelevinsky, Quantum Physics Volume 1: From Basics ti Symmetries and Perturbations. WILEY-VCH, 2011.

[35] V. B. Beresrerskii, E. M. Lifshitz, and L. P. Pitaevskii, Quantum electrodynamics. Butterworth-Heinemann, 1982. 
[36] A. Corney, Atomic and Laser Spectroscopy. Oxford, 1977.

[37] E. Arimondo, M. Inguscio, and P. Violino, "Experimental determinations of the hyperfine structure in the alkali atoms," Reviews of Modern Physics, vol. 49, no. 31, 1977.

[38] K. G. Woodgate, Elementary Atomic Structure. Oxford University Press, 1999.

[39] L. Armstrong, Theory of the Hyperfine Structure of Free Atoms. Wiley, 1 ed., 1971.

[40] D. A. Stec, "Rubidium 87 d line data." Oregon Center for Optics and Department of Physics, University of Oregon, 2008.

[41] D. A. Stec, "Rubidium 85 d line data." Oregon Center for Optics and Department of Physics, University of Oregon, 2008.

[42] J. Weiner and P. T. Ho, Light-Matter Interaction: Fundamentals and Applications, vol. 1. Willey, 2003.

[43] P. Meystre, Atom Optics. 1, Springer, 2001.

[44] J. Weiner, Cold and Ultracold collisions in Quantum Microscopic and Mesoscopic Systems. 1, Cambridge, 2003.

[45] J. Weiner and P. T. Ho, Light-Matter Interaction: Fundamentals and Application V.1, vol. 1 of 1. Willey, 2003.

[46] S. Chu, "The manipulation of neutral particles." Nobel Lecture, 1997.

[47] C. C. Tannoudje, "Manipulating atoms with photons." Nobel Lecture, 1997.

[48] W. D. Philips, "Laser cooling and trapping of neutral atoms." Nobel Lecture, 1997.

[49] B. Simons, "Lectures 12-14, atomic structure and multi-electron atoms." Cambridge Lecture on Advanced Quantum Mechanics, 2009.

[50] W. D. Philips, "Laser cooling and trapping of neutral atoms," Reviews of Modern Physics, vol. 70, no. 3, pp. 721-741, 1998. 
[51] S. Chu, L. Hollberg, J. E. Bjorkholm, A. Cable, and A. Ashkin, "Three-dimensional viscous confinement and cooling of atoms by resonance radiation pressure," Reviews of Modern Physics, vol. 55, no. 3, pp. 48-51, 1985.

[52] H. Perrin, "Doppler cooling and magneto-optical trapping." Les Houches lectures on laser cooling and trapping, 2002.

[53] B. Stiller, U. Seyfarth, and G. Leuchs, "Temporal and spectral properties of quantum light." 2014.

[54] E. S. Muckley, "Constructing a magneto-optical trap for cold atom trapping," Master's thesis, California Polytechnic State University, 2009.

[55] C. Awe, "Magneto optical trapping of rb 87," Master's thesis, University of Colorado, Boulder, 2015.

[56] M. L. Harris, Realisation of a Cold Mixture of Rubidium and Caesium. PhD thesis, Department of Physics, Durham University, 72008.

[57] H. J. Metcalf and P. van der Straten, Optical Molasses. Springer New York, 1 ed., 1999.

[58] J. Dalibard and C. Cohen-Tannoudji, "Laser cooling below the doppler limit by polarization gradients:simple theoretical models," J. Opt. Soc. Am, vol. 6, no. 11, pp. 2023-2045, 1989.

[59] P. J. Ungar, D. S. Weiss, E. Riis, and S. Chu, "Optical molasses and multilevel atoms: theory," J. Opt. Soc. Am. B, vol. 6, no. 11, pp. 2058-2071, 1989.

[60] A. R. Gorges, "Simultaneous trapping of ${ }^{85} r b$ and ${ }^{87} r b$ in a far off resontant trap," Master's thesis, 2010.

[61] R. Paschotta, Doppler cooling. https://www.rp-photonics.com/encyclopedia_ cite.html?article=Doppler\%20 cooling. 
[62] D. Stamper-Kurn, "Lecture physics 111-lab: Atom trapping mot." University of California at Berkeley, 2012.

[63] S. Patel, A Chirped, Pulsed Laser System and Magneto-Optical Trap for Rubidium. PhD thesis, University of Southampton, 12009.

[64] C. T. Whelan and C. T. Whelan, New Directions in Atomic Physics (Physics of Atoms and Molecules). Springer, 1999.

[65] M. H. T. Extavour, "Design and construction of magnetic elements for trapping and transport of cold neutral atoms," Master's thesis, 2004.

[66] I. H. Benzaquen, Degenerate quantum gases production and coherent manipulation on atom chips. PhD thesis, Universidad Autonoma de Madrid, European Laboratory for non-linear spectroscopy (LENS), 72012.

[67] J. Dalibard and C. Cohen-Tannoudji, "Laser cooling below the doppler limit by polarization gradients:simple theoretical models," J. Opt. Soc. Am. B, vol. 6, no. 11, pp. 2023-2046, 1989.

[68] D. Müller, D. Z. Anderson, R. Grow, P. D. Schwindt, and E. A. Cornell, "Guiding neutral atoms around curves with lithographically patterned current-carrying wires," Phys. Rev. Lett, vol. 83, no. 5194, 1999.

[69] D. Müller, E. A. Cornell, M. Prevedelli, P. D. D. Schwindt, Y.-J. Wang, and D. Z. Anderson, "Magnetic switch for integrated atom optics," Phys. Rev. A, vol. 63, 2001.

[70] D. Müller, E. Cornell, M. Prevedelli, P. Schwindt, A. Zozulya, and D. Anderson, "Waveguide atom beam splitter for laser-cooled neutral atoms," Opt. Lett., vol. 25, pp. $1382-1384,2000$.

[71] H. Ott, J. Fortagh, G. Schlotterbeck, A. Grossmann, and C. Zimmermann, "Boseeinstein condensation in a surface microtrap," Phys. Rev. Lett., vol. 87, no. 230401, 2001. 
[72] S. Bartalini, Magnetic microtraps for Bose-Einstein Condensation. PhD thesis, Universita Degli Studi Di Firenze, 2005.

[73] Y. B. Ovchinnikov, I. Manek, and R. Grimm, "Surface trap for cs atoms based on evanescent-wave cooling," Phys. Rev. Lett., vol. 79, no. 2225, 1997.

[74] H. Gauck, M. Hartl, D. Schneble, H. Schnitzler, T. Pfau, and J. Mlynek, "Quasi-2d gas of laser cooled atoms in a planar matter waveguide," Phys. Rev. Lett., vol. 81, no. 5298,1998 .

[75] K. I. Lee, J. A. Kim, H. R. Noh, and W. Jhe, "Single-beam atom trap in a pyramidal and conical hollow mirror," Opt. Lett., vol. 21, no. 15, pp. 1177-1179, 1996.

[76] J. A. Kim, K. I. Lee, H. R. Noh, W. Jhe, and M. Ohtsu, "Atom trap in an axicon mirror," Opt. Lett., vol. 22, no. 2, pp. 117-119, 1997.

[77] R. S. Williamson, P. A. Voytas, R. T. Newell, and T. Walker, "A magneto-optical trap loaded from a pyramidal funnel," Opt. Exp., vol. 3, no. 3, pp. 111-117, 1998.

[78] J. Reichel, W. Hansel, and T. W. Hänsch, "Atomic micromanipulation with magnetic surface trapsl," Phys. Rev. Lett, vol. 83, no. 3398, 1999.

[79] R. Folman, P. Krüger, D. Cassettari, B. Hessmo, T. Maier, and J. Schmiedmayer, "Controlling cold atoms using nanofabricated surfaces: Atom chips," Phys. Rev. Lett, vol. 84, no. 4749, 2000.

[80] Kimball, Physics, and MDC, 6.0" Spherical Octagon - Vacuum Chamber. http: //www.kimballphysics.com/mcf600-sphoct-f2c8.

[81] D. Müller, D. Z. Anderson, R. J. Grow, P. D. D. Schwindt, and E. A. Cornell, "Guiding neutral atoms around curves with lithographically patterned current-carrying wires," Phys. Rev. Lett., vol. 83, pp. 5194-5197, 121999.

[82] D. Müller, E. A. Cornell, M. Prevedelli, P. D. D. Schwindt, Y.-J. Wang, and D. Z. 
Anderson, "Magnetic switch for integrated atom optics," Phys. Rev. A., vol. 63, 3 2001.

[83] D. Müller, E. A. Cornell, M. Prevedelli, P. D. D. Schwindt, A. Zozulya, and D. Z. Anderson, "Waveguide atom beam splitter for laser-cooled neutral atoms," Opt. Lett., vol. 25, pp. 1382-1384, 92000.

[84] J. Jackson, Classical Electrodynamics. Wiley, 1998.

[85] T. Bergeman, G. Erez, and H. Metcalf, "Magnetostatic trapping fields for neutral atoms.," Phys. Rev. A, vol. 35, no. 4, p. 1535, 1987.

[86] L. Yu and L. Qiang, "The analysis of uniformity of the rectangular helmholtz coils magnetic field." Rectangular Helmholtz coils, 2005.

[87] ESRF, Radia software package. http://www.esrf.eu/Accelerators/Groups/ InsertionDevices/Software/Radia/Documentation/Introduction.

[88] T. Tekin, M. Töpper, and H. Reichl, eds., "PICSIP: New System-in-Package Technology Using A High Bandwidth Photonic Interconnection Layer For Converged Microsystems", vol. 7366 of 736618 , Proc. SPIE, 2009.

[89] C. Peng and W. A. Challener, "Input-grating couplers for narrow gaussian beam: influence of groove depth," Optical Society of America, 2004.

[90] B. Jalali and S. Fathpour, "Silicon photonics," Journal Of Lightwave Technology, vol. 24, pp. 4600-4615, 122006.

[91] G. T. Reed and A. P. Knights, Silicon Photonics and introduction. Wiley, 2004.

[92] B. L. Weiss and R. A. Soref, "Introduction to the issue on silicon-based optoelectronics," IEEE Journal of Selected Topics in Quantum Electronics, vol. 4, 121998.

[93] J. M. Senior, Optical Fiber Communications: Principles and Practice. Prentice-Hall, London, 2 ed., 1992. 
[94] J. Nayyer, Y. Suematsu, and H. Tokiwa, "Mode coupling and radiation loss of clad type optical waveguides due to the index inhomogeneities of the core material," Opt. Quant. Electron., vol. 7, pp. 481-492, 1975.

[95] P. K. Tien, "Light waves in thin films and integrated optics," Appl. Opt., vol. 10, pp. 2395-2413, 1971.

[96] R. G. Hunsperger, Integrated Optics: Theory and Technology. Springer-Verlag, Berlin, 3 ed., 1991.

[97] T. S. Moss, G. J. Burell, and B. Ellis, Semiconductor Opto-electronics. Butterworth London, 1973.

[98] R. A. Soref, J. Schmidtchen, and K. Petermann, "Large single-mode rib waveguides in gesi-si and si-on-so2," IEEE Journal Of Quantum Electronics, vol. 27, pp. 19711974, 81991.

[99] S. P. Pogossian, L. Vescan, and A. Vonsovici, "The single-mode condition for semiconductor rib waveguides with large cross section," Journal Of Lightwave Technology, vol. 16, pp. 1851-1853, 101998.

[100] O. G. de Villasante, "Design and simulation of vertical grating coupler for photonic integrated system-in-package," 42010.

[101] Q. Wang and S. T. Ho, "Modal analysis of silicon photonic wire and tm pass nanowaveguide polarizer," (PhotonicsGlobal@Singapore), pp. 1-4, IEEE, 122008.

[102] Y. A. Vlasov and S. J. McNab, "Waveguiding in silicon-on-insulator photonic crystal and single mode strip waveguides," vol. 2, (The 17th Annual Meeting of the IEEE Lasers and Electro-Optics Society), pp. 809-810, IEEE, 112004.

[103] Y. A. Vlasov and S. J. McNab, "Losses in single-mode silicon-on-insulator strip waveguides and bends," Optical Society of America, 2004.

[104] W. Bogaerts, R. Baets, P. Dumon, V. Wiaux, S. Beckx, D. Taillaert, B. Luyssaert, 
J. V. Campenhout, P. Bienstman, and D. V. Thourhout, "Nanophotonic waveguides in siliconon-insulator fabricated with cmos technology," Journal Of Lightwave Technology, vol. 23, pp. 401-412, 12005.

[105] T. Tsuchizawa, K. Yamada, H. Fukuda, T. Watanabe, J. Takahashi, M. Takahashi, T. Shoji, E. Tamechika, S. Itabashi, and H. Morita, "Microphotonics devices based on silicon microfabrication technology," IEEE Journal Of Selected Topics In Quantum Electronics, vol. 11, pp. 232-240, 12005.

[106] L. Vivien, F. Grillot, E. Cassan, D. Pascal, S. Lardenois, A. Lupu, S. Laval, M. Heitzmann, and J. M. Fedeli, "Comparison between strip and rib soi microwaveguides for intra-chip light distribution," Optical Materials 27 (2005), pp. 756-762, 102004.

[107] C. A. Barrios, V. R. Almeida, R. Panepucci, and M. Lipson, "Electrooptic modulation of silicon-on-insulator submicrometer-size waveguide devices," Journal Of Lightwave Technology, vol. 21, pp. 2332-2339, 102003.

[108] D. V. Thornhout, P. Dumon, W. Bogaerts, G. Roelkens, D. Taillaert, G. Priem, and R. Baets, "Recent progress in soi nanophotonic waveguides," ECOC 2005 Proceedings, vol. 2, pp. 241-244, 2005.

[109] J. C. Wirth, "Silicon grating couplers for low loss coupling between optical fiber and silicon nanowires," 122011.

[110] V. Nguyen, T. Montalbo, C. Manolatou, A. Agarwal, C. yin Hong, J. Yasaitis, L. C. Kimerling, and J. Michel, "Silicon-based highly-efficient fiber-to-waveguide coupler for high index contrast systems," Appl. Phys. Lett., vol. 88, 22006.

[111] T. Tsuchizawa, K. Yamada, H. Fukuda, T. Watanabe, J. ichi Takahashi, M. Takahashi, T. Shoji, E. Tamechika, S. ichi Itabashi, and H. Morita, "Microphotonics devices based on silicon microfabrication technology," IEEE Journal of Selected Topics in Quantum Electronics, vol. 11, pp. 232-240, 22005. 
[112] V. R. Almeida, R. R. Panepucci, and M. Lipson, "Nanotaper for compact mode conversion," Optics Letter, vol. 28, pp. 1302-1304, 82003.

[113] Z. Wang, "Design and simulation of spot size converter for photonic integrated system in package," 42010.

[114] M. L. Dakes, "Grating coupler for efficient excitation of optical guided waves in thin films," App. Phys. Lett., vol. 16, pp. 523-525, 61970.

[115] R. Waldhausl, B. Schnabel, P. Dannberg, E.-B. Kley, A. Brauer, and W. Karthe, "Efficient coupling into polymer waveguides by gratings," Applied Optics, vol. 36, pp. 9383-9390, 121997.

[116] J. H. Harris, R. K. Winn, and D. G. Dalgoutte, "Theory and design of periodic couplers," Applied Optics, vol. 11, pp. 2234-2241, 101972.

[117] V. A. Sychugov, A. V. Tischenko, B. A. Usievich, and O. Parriaux, "Optimization and control of grating coupling to or from a silicon-based optical waveguide," Optical Engineering, vol. 35, pp. 3092-3100, 111996.

[118] D. Taillaert, Grating couplers as Interface between Optical Fibres and Nanophotonic Waveguides. PhD thesis, Ghent University, 62004.

[119] L. Zimmermann, M. Schnarrenberger, T. Mitze, J. Bruns, and K. Petermann, "Study of reactive ion etched (rie) facets of silicon-on-insulator (soi) rip waveguides," in IEEE international SOI conference proceedings, pp. 15-16, 101998.

[120] X. C. Tong, Advanced Integrated Optical Waveguide, vol. 46. Springer, 2014.

[121] W. H. P. Pernice, C. Xiong, and H. X. Tang, "High q micro-ring resonators fabricated from polycrystalline aluminum nitride films for near infrared and visible photonics," Opt. Exp., vol. 10, pp. 12261-12269, 52012. 


\title{
Optimal preparation of quantum states on an atom chip device
}

\author{
C. Lovecchio ${ }^{1}$, F. Schäfer ${ }^{1,4}$, S. Cherukattil ${ }^{1}$, M. Alì Khan ${ }^{1}$, I. Herrera ${ }^{1,6}$ and F. S. Cataliotti ${ }^{1,2,3}$, \\ T. Calarco ${ }^{5}$, S. Montangero ${ }^{5}$, F. Caruso ${ }^{1,2,3}$ \\ ${ }^{1}$ LENS and Università di Firenze, Via Nello Carrara 1, 50019 Sesto Fiorentino, Italy \\ ${ }^{2}$ Dipartimento di Fisica ed Astronomia, Università di Firenze, Via Sansone 1, 50019 Sesto Fiorentino, Italy \\ ${ }^{3}$ QSTAR, Largo Enrico Fermi 2, 50125 Firenze, Italy \\ ${ }^{4}$ Department of Physics, Graduate School of Science, Kyoto University, 606-8502 Kyoto, Japan \\ ${ }^{5}$ Institut für Quanteninformationsverarbeitung \& IQST, \\ Universität Ulm, Albert-Einstein-Allee 11, D-89069 Ulm, Germany and \\ ${ }^{6}$ Centre for Quantum and Optical Science, Swinburne University of Technology, Melbourne, Australia 3122
}

\begin{abstract}
Atom chips provide compact and robust platforms towards practical quantum technologies. A quick and faithful preparation of arbitrary input states for these systems is crucial but represents a very challenging experimental task. This is especially difficult when the dynamical evolution is noisy and unavoidable setup imperfections have to be considered. Here, we experimentally prepare with very high small errors different internal states of a Rubidium Bose-Einstein condensate realized on an atom chip. As a possible application of our scheme, we apply it to improve the sensitivity of an atomic interferometer.
\end{abstract}

Microscopic magnetic traps, as atom chips [1, 2], represent an important advance in the field of degenerate quantum gases towards their realization of practical quantum technological devices. Indeed, applications outside the laboratory depend on the compactness and robustness of these setups. Atom chips have already enabled, for instance, the demonstration of miniaturized interferometers $[3,4]$, or the creation of squeezed atomic states [5]. Very recently, they have been also applied to the realization of atom interferometers based on non-classical motional states [6]. Furthermore, atom chips can be integrated with nanostructures [7], or photonic components [8], to implement new quantum information processing tools, or used as novel platforms for quantum simulations [9] and controllable coherent dynamics [10].

As in most quantum technological applications, the ability to quickly and faithfully prepare arbitrary input states is of utmost importance. In particular, it is essential to speedup the initialization protocol of these quantum devices in order to reduce the effects of the inevitably present noise and decoherence sources. In this context, quantum optimal control provides powerful tools to tackle this problem by finding the optimal way to transform the system from an experimentally readily prepared initial condition to a desired state with high fidelity from which further quantum manipulations can be performed [11-13]. It has been successfully implemented in several physical systems, ranging from cold atoms [14] to molecules [15]. Recently optimal control algorithms have been developed and successfully applied to many-body quantum systems [16-21].

In addition, optimal control allows one to speed up the state preparation process up to the ultimate bound imposed by quantum mechanics, the so-called quantum speed limit (QSL) [22-25]. Indeed, the change of a state into a different one cannot occur faster than a time scale that is inversely proportional to the associated energy scale. This is especially relevant for quantum systems like Bose-Einstein condensates (BECs) where it is crucial to perform some desired (coherent) task before dephasing processes unavoidably occur [26].

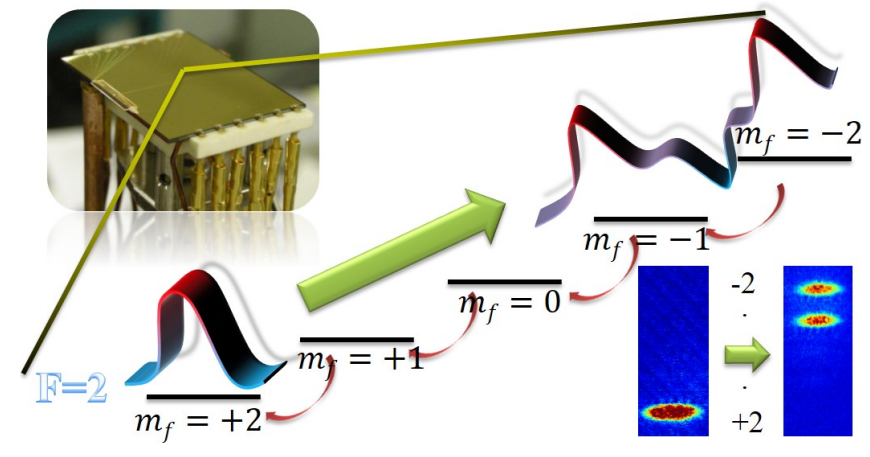

FIG. 1. Pictorial representation of the preparation of the internal states of a Bose-Einstein condensate realized on a Atom chip. In the bottom right corner we show the experimentally observed clouds of atoms that are initially in level +2 and then halved in the levels -1 and -2 via optimal control.

In this work we present experimental results obtained on a Rubidium $\left({ }^{87} \mathrm{Rb}\right) \mathrm{BEC}$, trapped on an atom chip, evolving in a five-level Hilbert space given by the five spin orientations of the $F=2$ hyperfine ground states - see Fig. 1 . We use optimal control theory to design frequency modulated radiofrequency $(\mathrm{RF})$ pulses that, starting from a given initial state fixed by the BEC evaporation procedure, prepare different arbitrary coherent superpositions of states with high precision. We also show how the latter scales with the preparation time, with a highly satisfactory agreement between theory and experiment. We apply, finally, this technique to improve the sensitivity of an atom interferometer.

Model: In the presence of a magnetic field the degeneracy between the spin orientations of an atomic hyperfine state is lifted. The phenomenon is described by the Breit-Rabi formula which determines the energies of all different sublevels. For the five fold $F=2$ hyperfine ground state of ${ }^{87} \mathrm{Rb}$ in the presence of a constant magnetic bias field arbitrarily set to $6.179 \mathrm{G}$ [27], the atomic part of the Hamiltonian is $H_{0}=\hbar \operatorname{diag}\left(\omega_{1}, \omega_{2}, \omega_{3}, \omega_{4}, \omega_{5}\right)=$ 
$2 \pi \hbar \operatorname{diag}(8635,4320,0,-4326,-8657) \mathrm{kHz}$, where the state basis is chosen to go from $m_{F}=+2$ to $m_{F}=-2$ and the energy values have been shifted to set $\omega_{3}=0$. Under the effect of an RF field it is convenient to write the evolution of this system in the Rotating Wave Approximation (RWA). Let us define the terms $V(t)=\hbar f(t) \operatorname{diag}(-2,-1,0,1,2)$ and

$$
H_{1}=\hbar\left(\begin{array}{ccccc}
0 & \Omega & 0 & 0 & 0 \\
\Omega & 0 & \sqrt{3 / 2} \Omega & 0 & 0 \\
0 & \sqrt{3 / 2} \Omega & 0 & \sqrt{3 / 2} \Omega & 0 \\
0 & 0 & \sqrt{3 / 2} \Omega & 0 & \Omega \\
0 & 0 & 0 & \Omega & 0
\end{array}\right)
$$

where $f(t)=\frac{d}{d t}[t \omega(t)]$ describes the energy differences between the five $F=2$ levels within the RWA, $\omega(t)$ is the time dependent frequency of the coupling field that will be modulated in order to implement our optimal control scheme.The Rabi frequency $\Omega$ is proportional to the RF field intensity and, in all our experiments, we set $\Omega=2 \pi 60 \mathrm{kHz}$. Hence, the driving field Hamiltonian is $H_{1}+V(t)$.

In order to include the unavoidable presence of dephasing noise, mainly originated in our experiment by the presence of environmental magnetic noise superimposed on the bias field, we also add in our model a Lindblad superoperator term $\mathcal{L}$, acting on the density matrix $\rho$ as $\mathcal{L}(\rho)=$ $\sum_{j=1}^{5} \gamma_{j}[-\{|j\rangle\langle j|, \rho\}+2|j\rangle\langle j|\rho| j\rangle\langle j|]$, which randomizes the phase of each sublevel $j$ with a rate $\gamma_{j}$. For simplicity, we assume the same dephasing rate for all five sublevels, i.e. $\gamma_{j} \equiv \gamma$. The time evolution of the density matrix is then given by

$$
\frac{d}{d t} \rho(t)=-\frac{i}{\hbar}\left[H_{0}+H_{1}+V(t), \rho(t)\right]+\mathcal{L}(\rho(t)) .
$$

Optimal control: Here, we use a recently introduced versatile and efficient optimization algorithm (CRAB) [16, 17] for the time dependence $f(t)=f_{0}\left(1+\sum_{k=-n_{f}}^{n_{f}} A_{k} \exp \left(i \nu_{k} t\right)\right)$ with $\nu_{k}=2 \pi k / T$, $n_{f}$ being the number of frequencies, $A_{k}$ complex numbers, and $T$ the time duration of the modulation. The optimization is reflected in the modulation of the field's frequency $\omega(t)$, which we constrain within the range $\omega(t) \in 2 \pi[4000,4700]$ $\mathrm{kHz}$ in order to keep the Rabi frequency $\Omega$ constant (see Supplementary Information for further details). The optimization of the control parameters $\left(A_{k}\right)$ in $f(t)$ is done by means of the Subplex variant of the Nelder-Mead algorithm [29]. The error function to be minimized is $\epsilon=\sum_{i}\left|\rho_{i i}-b_{i}\right| / 2$, with $\rho_{i i}$ being the final population (after time $T$, i.e. at the end of the pulse) of the sublevel $i$, while $b_{i}$ are the corresponding target populations we want to achieve. The initial state $\rho_{0}$ is given by all population in the energy level $m_{f}=+2$, i.e. $\rho_{11}(t=0)=1$. In the left half of Tab. I, we show the target populations of different target states $(\mathrm{A}, \ldots, \mathrm{I})$. Note that there are no constraints on the coherence terms (i.e., the off-diagonal elements $\rho_{i j}$ ), which are however relevant in the context of quantum state preparation. Even if state tomography would have been in principle possible [28], it would have been hard to perform it for all cases studied here. For this reason, in the experiment we measure only the populations of the five levels, while in the numerical simulations we take into account the full density matrix during the optimization. However, we have strong experimental evidence that the coherence terms (i.e. relative phases) are also prepared according to our theoretical predictions, because: 1) as shown in the fine details of the inset of Fig. 3, there is a very good agreement between theory and experiment in the population dynamical evolution, 2) when we prepare eigenstates of the Hamiltonian (see Fig. 2 and in the Supplementary Information Fig. 6), they do not evolve anymore, as expected if the Hamiltonian is kept fixed, 3) finally, we consider a multi-state interferometer and our theoretical results are reproduced very well by the experimental data. These three tests would have failed if all the relative phases had not been prepared as in the target quantum state that is considered in the numerical optimization protocol.

Experimental setup: We have implemented experimentally the optimal control protocols on a BEC of ${ }^{87} \mathrm{Rb}$ atoms produced in a magnetic micro-trap realized on an atom chip. The trap has a longitudinal frequency of $46 \mathrm{~Hz}$ while the radial trapping frequency is $950 \mathrm{~Hz}$. The atoms are evaporated to quantum degeneracy by ramping down the frequency of a radio frequency $(\mathrm{RF})$ field. The $\mathrm{BEC}$ has typically $8 \cdot 10^{4}$ atoms, at a critical temperature of $0.5 \mu \mathrm{K}$ and is $300 \mu \mathrm{m}$ from the chip surface. The magnetic fields for the micro-trap are provided by a Z-shaped wire on the atom chip and an external pair of Helmholtz coils. The RF fields for evaporation and manipulation of the Zeeman states are produced by two additional conductors also integrated on the atom chip. Finally the homogeneous bias field that lifts the degeneracy between the spin orientations is created by two further external Helmholtz coils $[10,30]$.

All manipulations are performed $0.7 \mathrm{~ms}$ after turning off the magnetic trap to guarantee bias field homogeneity and strongly reduce the effects of atomic collisions that can produce decoherence. In this way the most relevant source of noise on the evolution turns out to be the instability of the environmental magnetic field, which has a spectrum distribution made of several peaks on a decreasing envelope in the low frequency range. To record the number of atoms in each of the $m_{F}$ states of the $F=2$ hyperfine state we apply a Stern-Gerlach method. After the state manipulation has been performed, in addition to the homogeneous bias field, an inhomogeneous magnetic field is applied along the quantization axis for $10 \mathrm{~ms}$. This causes the different $m_{F}$ states to spatially separate. After a time of $23 \mathrm{~ms}$ of expansion a standard absorption imaging sequence is performed. The atomic populations in each $m_{F}$ state is normalized to the total number of observed atoms.

Results: Let us define the theoretical state preparation error $\epsilon_{T}$ and the experimentally measured one $\epsilon_{E}$. We then apply ten times the same preparation pulse and the reported values of $\epsilon_{E}$ are the corresponding means and standard deviations. We take into account the presence of magnetic noise in 


\begin{tabular}{|c|c|c|c|c|c||c|c|c|}
\hline Target State & $\rho_{11}$ & $\rho_{22}$ & $\rho_{33}$ & $\rho_{44}$ & $\rho_{55}$ & $\epsilon_{T}$ & $\epsilon_{E}$ & $\mathcal{F}$ \\
\hline $\mathrm{A}$ & $1 / 2$ & 0 & 0 & 0 & $1 / 2$ & $0.04(3)$ & $0.07(1)$ & 0.71 \\
\hline $\mathrm{B}$ & $1 / 2$ & 0 & 0 & $1 / 2$ & 0 & $0.04(2)$ & $0.02(1)$ & 0.67 \\
\hline $\mathrm{C}$ & 0 & $1 / 2$ & 0 & $1 / 2$ & 0 & $0.04(3)$ & $0.04(1)$ & 0.11 \\
\hline $\mathrm{D}$ & $1 / 2$ & $1 / 2$ & 0 & 0 & 0 & $0.03(2)$ & $0.02(1)$ & 0.71 \\
\hline $\mathrm{E}$ & 0 & $1 / 3$ & $1 / 3$ & $1 / 3$ & 0 & $0.04(2)$ & $0.03(1)$ & 0.02 \\
\hline $\mathrm{F}$ & $1 / 5$ & $1 / 5$ & $1 / 5$ & $1 / 5$ & $1 / 5$ & $0.02(1)$ & $0.03(1)$ & 0.45 \\
\hline $\mathrm{G}$ & 0 & 1 & 0 & 0 & 0 & $0.05(4)$ & $0.04(1)$ & 0.15 \\
\hline $\mathrm{H}$ & 0 & 0 & 0 & 1 & 0 & $0.04(3)$ & $0.03(1)$ & 0.07 \\
\hline $\mathrm{I}$ & 0 & 0 & 1 & 0 & 0 & $0.07(3)$ & $0.07(1)$ & 0.15 \\
\hline
\end{tabular}

TABLE I. Target populations $\rho_{i i}$ of different target states $(\mathrm{A}, \ldots, \mathrm{I})$, theoretical $\left(\epsilon_{T}\right)$ and experimental $\left(\epsilon_{E}\right)$ error functions in their preparation, and fidelity $\mathcal{F}$ between the initial and the target state. The error bars for $\epsilon_{T}$ are obtained considering the maximal deviation when the noise rate $\gamma$ is varied in the range $\gamma \in[20,200] 2 \pi \mathrm{Hz}$ and the magnetic field in a $1 \mathrm{mG}$ range around the ideal value $B=6.179 \mathrm{G}$. In the absence of dephasing noise, the theoretically predicted $\epsilon_{T}$ are always smaller than 0.02 .

the experiment by including in the theoretical model an effective dephasing with rate $\gamma \in 2 \pi[20,200] \mathrm{Hz}$ (see Eq. 2).

In the right half of Tab. I we show the errors $\epsilon$ in the preparation of quantum states $(A, \ldots, I)$, with a highly satisfactory agreement between theory and experiment. We include also a measure of distance (Uhlmann fidelity $\mathcal{F}$ [31]) between the initial state $\rho_{0}$ and the target state $\rho_{T}$, which is defined as $\mathcal{F}\left(\rho_{0}, \rho_{T}\right)=\operatorname{Tr} \sqrt{\rho_{0}^{1 / 2} \rho_{T} \rho_{0}^{1 / 2}}$, in order to show that the target states do cover a large portion of the Hilbert space, i.e. they have varying distances from the initial state. Let us stress that these states cannot be prepared by simply using constant pulses [30].

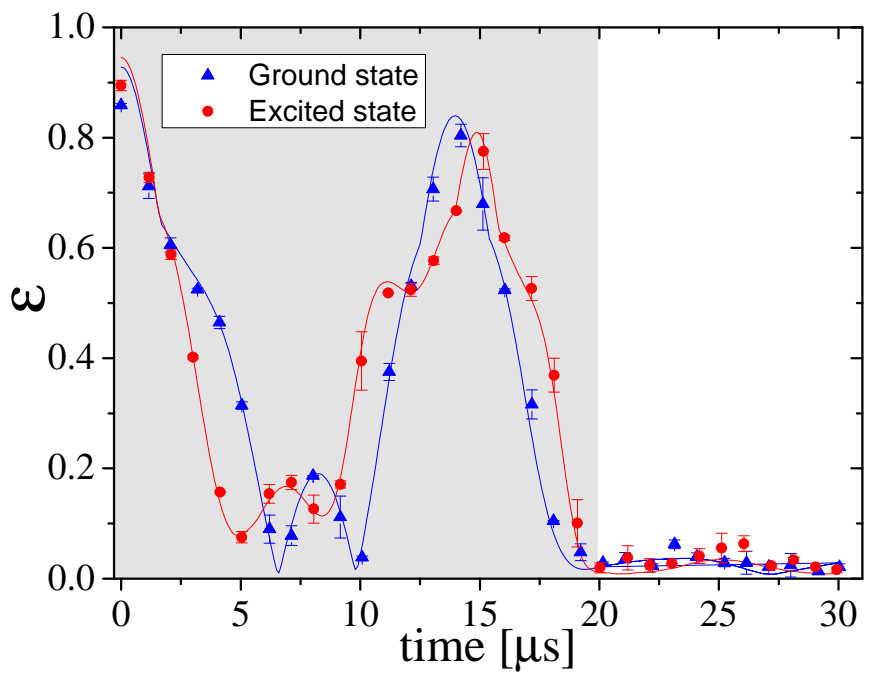

FIG. 2. Theoretical (lines) and experimental (dots) time evolution of $\epsilon$ for the preparation (grey area) of a ground state and the highest excited state of the Hamiltonian $H_{0}+H_{1}+\bar{V}(t)$ with $\overline{V(t)}$ being the harmonic potential that oscillates at $f(t)=\bar{f}=2 \pi 4323 \mathrm{kHz}$ for every $t \geq T=20 \mu s$ (white area).

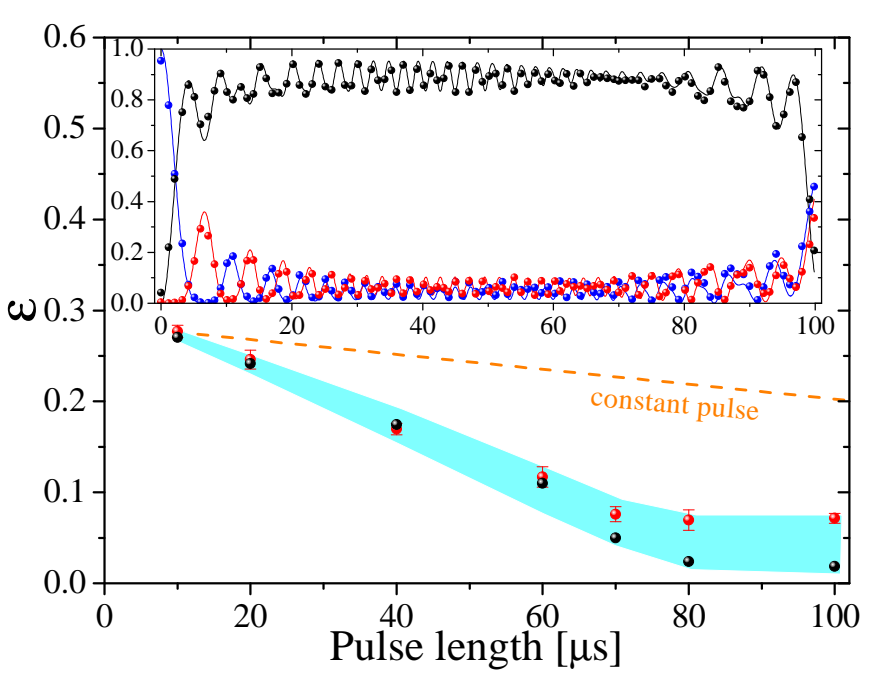

FIG. 3. State error preparation $\epsilon$ as a function of pulse length $T$, obtained theoretically (black dots), in the absence of dephasing noise, and experimentally (red dots), to prepare the state $A$ by optimal control. The effect of dephasing noise in the range $2 \pi[20,200] \mathrm{Hz}$ is also considered (shaded region). The results achievable with constant $f(t) \equiv \bar{f}=2 \pi 4323 \mathrm{kHz}$ are also shown (dashed line). Inset: Theoretical (lines) and experimental (dots) dynamical evolution of the population of the levels $m_{F}=2$ (blue), $m_{F}=-2$ (red), and the sum of the remaining three levels (black) during the application of optimal pulse of length $100 \mu s$.

As noted above, these experimental results only show that we are able to prepare different population distributions in the $F=2$ manifold. To directly verify our ability to prepare superposition of levels, we have prepared the ground state and highest excited state of the Hamiltonian $H_{0}+H_{1}+\bar{V}(t)$ in which $f(t)=\bar{f}=2 \pi 4323 \mathrm{kHz}$, and observed the absence of any evolution of the population distribution of these states after applying the preparation pulse, i.e. for $t \geq T$, while keeping $f(t)=\bar{f}$ ( $T=20 \mu \mathrm{s}$ is the pulse length). The results are reported in Fig. 2 where we plot the experimental points and expected theoretical behaviour. The theoretical prediction without any free parameter is shown as a continuous line. Some typical examples of optimal pulses are shown in the Supplementary Information.

We then proceed to analyse the time limits for the preparation of the states, in other words we are interested in keeping the pulse length as short as possible while still suppressing errors in the state preparation. For this analysis we choose the target state $A$, fixing different pulse times and minimizing the error function $\epsilon$ under the same constraint on magnetic field and Rabi frequency. In Fig. 3 we report the results obtained. The theoretical noiseless results saturate very close to vanishing errors for a pulse length of $90 \mu \mathrm{s}$. For the experimental results we used the same procedure outlined above to determine the error value. Experimental results are perfectly matched to the theoretical noiseless value at short pulse times but deviate for longer pulses, due to the increasing effect of the low frequency experimental noise on the magnetic field. 
If we include in the simulation the magnetic field and dephasing noise term fluctuations to correct all the theoretical predictions, we obtain a good agreement with the experimental observation. The precise matching between theory and experiment is also shown in the inset of Fig. 3 where we report the experimentally measured values of the atomic populations in the five levels during the application of the $100 \mu$ s-pulse. No fitting procedure was used to get the theoretical results (shown as continuous lines).

Application to interferometry: We have implemented an atomic Ramsey interferometer scheme using some of the state preparation pulses listed above. In a conventional Ramsey interferometer the application of a $\pi / 2$ pulse creates a balanced superposition of two atomic states separated by an energy difference $\Delta E$. After the pulse, the atoms are let free to evolve for a time $\tau$, during which the states acquire a phase difference $\Delta E / \hbar \tau$. Then, the application of a second $\pi / 2$ pulse maps this phase difference onto the atomic populations, which then oscillate as $\cos (\Delta E / \hbar \tau)$. Even if the smallest signal that an interferometer can ultimately resolve is limited by the signal to noise ratio, typically its sensitivity is determined by the maximum slope of the interferometric fringe. The best achievable sensitivity of a conventional Ramsey interferometer is found to improve linearly with $\Delta E$.

The presence of five levels in the $F=2$ manifold complicates the analysis of the interferometer albeit offering a possibility to realise a multi-state interferometer with increased sensitivity [30]. Whereas optimal control would allow to maximize the performance of an interferometer [6], for our system this would require an extensive analysis of the content of information of the interferometric output that goes beyond the focus of the present work. Nonetheless, as an application of our protocol, we restrict ourselves to interferometric configurations where, for simplicity, only two levels play a role during the free evolution. To this aim, we use the state preparation pulses A, B, C, D (all of them populating two initial states only), both as input and output beam splitters.

For each preparation pulse we record the interferometric pattern, i.e. the behaviour of the relative populations in the $F=2$ manifold after applying the two pulses and a variable time delay $\tau$ between them. Among the five components of the interferometer's signal we select the one with the highest fringe amplitude $A$ and we calculate the point-to-point slope selecting its maximum value $S$. Since the fringe amplitude can be different for each preparation pulse, we take into account its effect on the sensitivity by plotting in Fig. 4 the values $S / A$ with respect to the energy difference $\Delta E$ between the levels populated via the state preparation pulse. The results confirm, within the errors, the expected linear dependency between the two quantities. Let us point out that none of these interferometers can be realized without optimal control since no one of the states above (A, B, C, D) can be reached with constant pulses. It must be noted that, in our experiment, we have not taken into consideration the fringe amplitude which could be manipulated by a suitable choice of optimization parameters. However, this simple application still allows us to

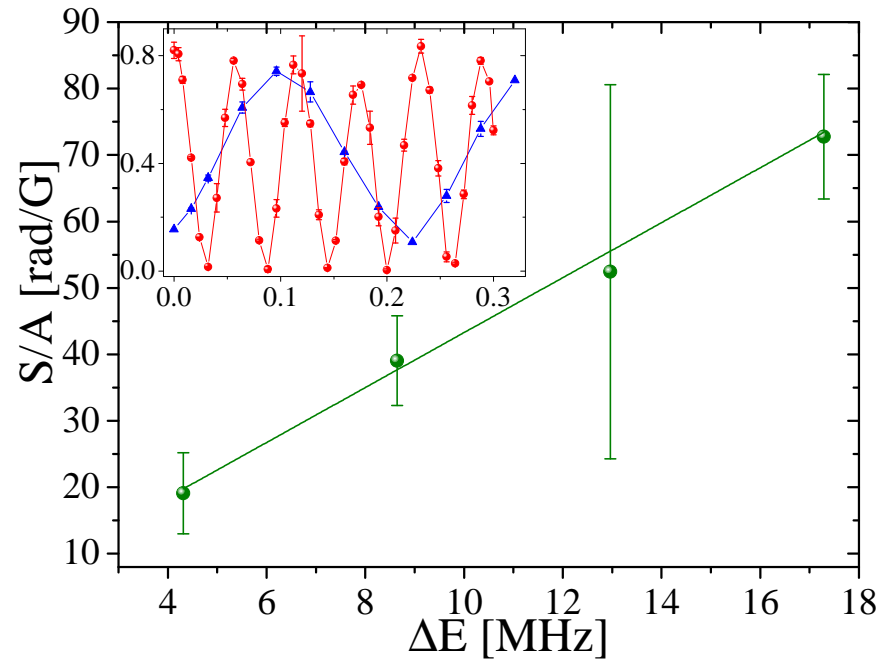

FIG. 4. Interferometer sensitivity $S$, calculated as the maximum slope of the fringe with the largest amplitude $A$, normalized with respect to the latter. The quantity $S / A$ is plotted as a function of the energy gap $\Delta E$ between the two levels that are initially populated by the optimal control preparation pulse to show the linear dependence between them. Inset: Relative populations on the level $m_{f}=+2$ for the state preparation pulses A (red circles) and D (blue triangles) vs. time $(\mu s)$.

achieve a four-fold improvement of the interferometer sensitivity, without changing neither the applied magnetic field nor the time interval between the two pulses.

Conclusions: The preparation of specific quantum states is a crucial step to control atom-chip devices, especially in the context of quantum information and communication technologies. However, this is highly non-trivial to perform even theoretically, for instance for complex quantum systems, and especially from the experimental side since one has to deal with imperfections and the presence of noise due to the external environment, limiting the observation time scale. Over the last years, the theory of optimal control has allowed to address this issue in an efficient way, also when working with fragile quantum system and non-unitary (noisy) evolutions. Here, we have applied optimal control tools to preparare a family of different internal states of a Rubidium Bose-Einstein condensate produced in an atom chip-based micro-trap. In particular, we theoretically and experimentally find that these states can be prepared with a very small error $(\epsilon \sim 0.03)$, with very short (control) pulses successfully overcoming the unavoidable presence of dephasing noise. Hence, we also test these protocols to implement improved interferometric configurations that would have been not accessible without the preparation of some input quantum states by optimal control. Therefore, these results might pave the way for new schemes towards, among others, better control of quantum dynamics, preparation of squeezed states, time-inversion of the system dynamics, realization of quantum gates, and so more powerful quantum information protocols. 
This work was supported by the Seventh Framework Programme for Research of the European Commission, under FET-Open grant MALICIA, QIBEC, SIQS, and by DFS via SFB/TRR21. The work of F.C. has been supported by EU FP7 Marie-Curie Programme (Career Integration Grant) and by MIUR-FIRB grant (RBFR10M3SB). We thank M. Inguscio for fruitful discussions and continuous support. QSTAR is the MPQ, LENS, IIT, UniFi Joint Center for Quantum Science and Technology in Arcetri.

\section{SUPPLEMENTARY INFORMATION}

As introduced in the main text, we use RF induced evaporation on magnetically trapped ${ }^{87} \mathrm{Rb}$ atoms to reach the BEC. The magnetic trap is provided by the chip structures and, before its loading, we perform optical pumping in the $m_{F}=2$ magnetic sublevel. This condensation scheme imply that the initial state of the condensate is the $\left|F=2, m_{F}=2\right\rangle$ state, so as initial condition every state preparation pulse uses a system in that pure state. After the BEC creation we let expand the atomic cloud in the homogeneous magnetic field for $0.7 \mathrm{~ms}$ and then, using a programmable function generator, we apply to a wire on the chip the signal $V(t)=V_{0} \sin [t \omega(t)]$, which build up the driving field. In the frequency range $[4000,4700]$ $\mathrm{Hz}$ in which we constrain $\omega(t)$ the radiation efficiency of the used wire can be considered constant, so the amplitude $V_{0}$ of the signal can be determined with high accuracy by performing a series of atomic population oscillation to have a Rabi frequency $\Omega=2 \pi 60 \mathrm{kHz}$.

To calibrate the homogeneous magnetic field applied by the

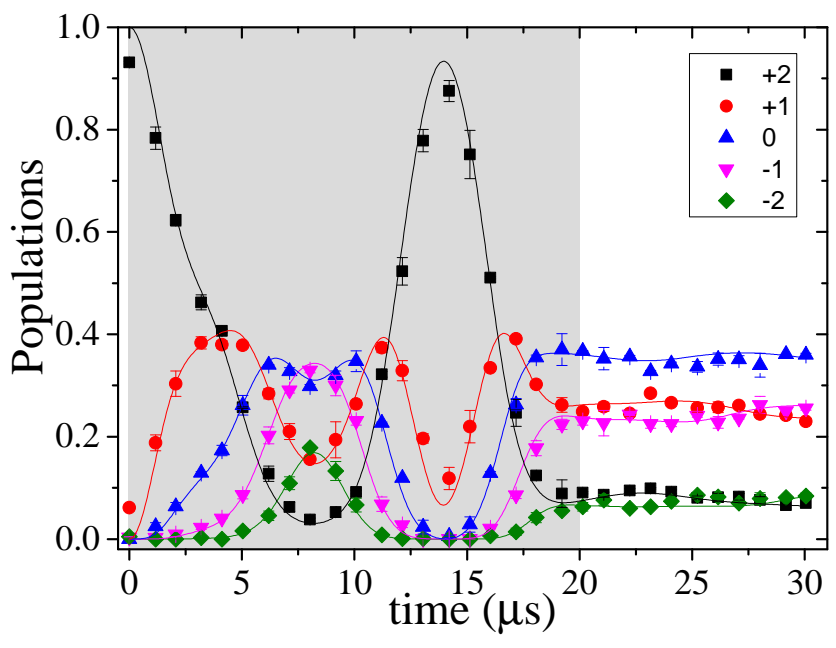

FIG. 5. Population behaviour of the five sublevels during the time evolution shown in Fig. 2 in the case of the ground state of the Hamiltonian $H_{0}+H_{1}+\bar{V}(t)$ with $\bar{V}(t)$ being the harmonic potential that oscillates at $f(t)=\bar{f}=2 \pi 4323 \mathrm{kHz}$, for both theory (lines) and experiment (dots). The gray area correspond to the evolution guided by the optimized pulse, and is followed by a constant frequency driving for every $t \geq T=20 \mu \mathrm{s}$, i.e. by the target Hamiltonian.

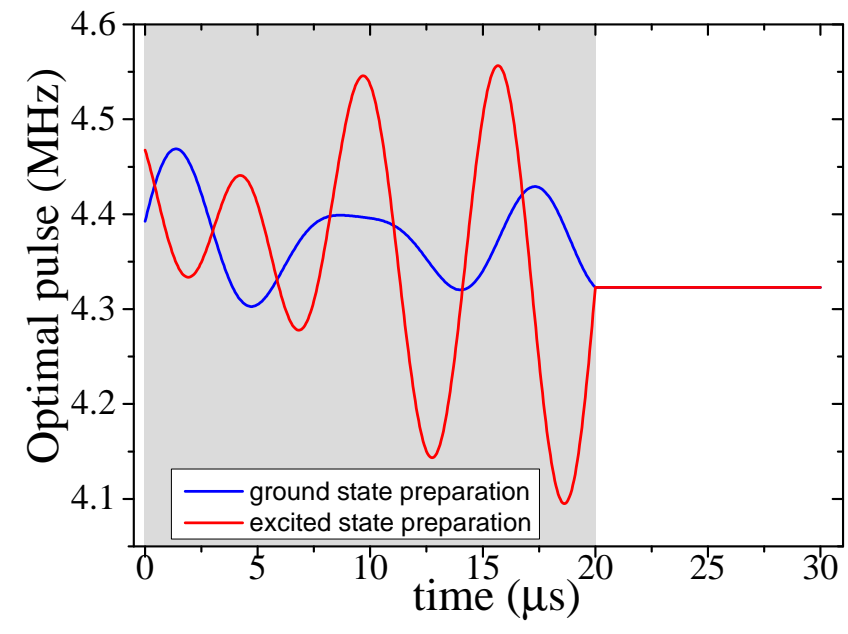

FIG. 6. Time evolution of the optimally shaped $f(t) /(2 \pi)$ for the preparation of the ground state and the excited state of the Hamiltonian $H_{0}+H_{1}+\bar{V}(t)$ with $\bar{V}(t)$ being the harmonic potential that oscillates at $f(t)=\bar{f}=2 \pi 4323 \mathrm{kHz}$. The optimally shaped pulse (gray area) is followed by a constant one for $t \geq T=20 \mu \mathrm{s}$.

Helmholtz coils, we close a multi-state atom interferometer with constant amplitude pulses as in [30] from which we deduce the right current to apply to the coils to produce the value $B_{t h}=6.179 \mathrm{G}$. To compensate for very slow variations of the magnetic field we repeat the calibration procedure before the sequence of ten measurements of a given state preparation pulse.

From a statistical analysis of the interferometer output we also derive information on the residual noise present in the field. In particular, by the reduction of the fringe contrast we deduce the dephasing rate interval $\gamma \in 2 \pi[20,200]$, while by the variations of the fringe phase we can estimate the amount of shot-to-shot offset field fluctuations, to $1 \mathrm{mG}$. Introducing these parameters in the theoretical model it is possible to derive also a maximal theoretical error by taking the worst and the best output of the model evolution. Those errors are reported in table I.

To observe the evolution during the optimization pulse, we splice it in subpulses, measuring the output at the end of each of them. Figure 5 shows an example of experimental points and theoretical prediction, reported in solid line, relative to the five population $m_{F}=+2 \ldots-2$ driven from the initial state to the ground state of the Hamiltonian $H_{0}+H_{1}+\bar{V}(t)$ with $\bar{V}(t)$ being the harmonic potential that oscillates at $f(t)=$ $\bar{f}=2 \pi 4323 \mathrm{kHz}$. The optimized preparation pulse is $20 \mu \mathrm{s}$ long and, after the preparation, we keep the frequency of the potential at the constant target value. The stability of the populations shows that the system is really in the target state, with the right coherence between the $\left|F, m_{F}\right\rangle$ components. Finally we show in Fig. 6 the optimal pulses for the preparation of the ground and excited states as in Fig. 5. Similar pulses (with $n_{f}=7$ ) have been obtained as a result of our optimization scheme to prepare the other states discussed in the main text. 
[1] R. Folman, J. Kruger, J. Schmiedmayer, J. Denschlag and C. Henkel, Adv. At. Mol. Opt. Phys. 48, 263 (2002).

[2] J. Förtagh and C. Zimmermann, Rev. Mod. Phys. 79, 235 (2007).

[3] Y-J. Wang, D.Z. Anderson, V.M. Bright, E.A. Cornell, Q. Diot, T. Kishimoto, M. Prentiss, R.A. Saravanan, S.R. Segal and S. Wu, Phys. Rev. Lett. 94, 090405 (2005).

[4] T. Schumm, S. Hofferberth, L.M. Andersson, S. Wildermuth, S Groth, I. Bar-Joseph, J. Schmiedmaye, and P. Krüge, Nat. Phys. 1, 57 (2005).

[5] M.F. Riedel, P. Böhi, Y. Li, T.W. Hänsch, A. Sinatra, and P. Treutlein, Nature 464, 1170 (2010).

[6] S. van Frank, A. Negretti, T. Berrada, R. Bucker, S. Montangero, J.-F. Schaff, T. Schumm, T. Calarco, and J. Schmiedmayer, in press in Nat. Commun. (2014), Eprint arXiv: 1402.0377

[7] M. Gierling, P. Schneeweiss, G. Visanescu, P. Federsel, M. Häffner, D. P. Kern, T. E. Judd, A. Günther,and J. Förtagh, Nat. Nanotechnol. 6, 446451 (2011).

[8] M. Kohnen, M. Succo, P.G. Petrov, R.A. Nyman, M. Trupke and E.A. Hinds, Nat. Photon. 535 (2011).

[9] S. Jose, P. Surendran, Y. Wang, I. Herrera, L. Krzemien, S. Whitlock, R. McLean, A. Sidorov, P. Hannaford, Phys. Rev. A 89, 051602(R) (2014).

[10] F. Schäfer, I. Herrera, S. Cherukattil, C. Lovecchio, F.S. Cataliotti, F. Caruso, A. Smerzi, Nat. Commun. 5, 3194 (2014).

[11] I. Walmsley and H. Rabitz, Phys. Today 56, 43 (2003).

[12] H. Mabuchi N. Khaneja, Internat. J. Robust Non-linear Control 15, 647-667 (2005)

[13] H. Rabitz, New. J. Phys. 11, 105030 (2009).

[14] S. Chu, Nature 416, 206-210 (2002).

[15] M. Shapiro and P. Brumer, J. Chem. Soc. Faraday Trans. 93, 1263 (1997).

[16] T. Caneva, T. Calarco, and S. Montangero, Phys. Rev. A 84,
022326 (2011).

[17] P. Doria, T. Calarco, and S. Montangero, Phys. Rev. Lett. 106, 190501 (2011).

[18] S. Rosi, et.al. Phys. Rev. A 88, 021601 (2013).

[19] J. Scheuer, X. Kong, R.S. Said, J. Chen, A. Kurz, L. Marseglia, J. Du, P.R. Hemmer, S. Montangero, T. Calarco, B. Naydenov, F. Jelezko, Eprint arXiv:1309.4399 (2013).

[20] F. Caruso, S. Montangero, T. Calarco, S.F. Huelga and M.B. Plenio, Phys. Rev. A 85, 042331 (2012).

[21] S. Hoyer, F. Caruso, S. Montangero, M. Sarovar, T. Calarco, M.B. Plenio and K.B. Whaley, New J. Phys. 16, 045007 (2014), special issue article in Focus on Coherent Control of Complex Quantum Systems.

[22] T. Caneva, M. Murphy, T. Calarco, R. Fazio, S. Montangero, V. Giovannetti, and G. E. Santoro, Phys. Rev. Lett. 103, 240501 (2009).

[23] T. Caneva, T. Calarco, R. Fazio, G.E. Santoro, and S. Montangero, Phys. Rev. A 84, 012312 (2011).

[24] V. Giovannetti, S. Lloyd, and L. Maccone, Phys. Rev. A 67, 052109 (2003).

[25] M. G. Bason, M. Viteau, N. Malossi, P. Huillery, E. Arimondo, D. Ciampini, R. Fazio, V. Giovannetti, R. Mannella, and O. Morsch, Nat. Phys. 8, 147 (2011).

[26] I. Bloch, J. Dalibard, and W. Zwerger, Rev. Mod. Phys. 80, 885964 (2008).

[27] The value of the field $B$ is chosen around $6 G$ so that it is much larger than the magnetic noise fluctuations but, at the same time, the current used to produce it is not high enough to cause significant heating of the coils.

[28] C. Gross, T. Zibold, E. Nicklas, J. Estéve and M. K. Oberthaler, Nature 464, 1165-1169 (2010)

[29] T. Rowan, "Functional Stability Analysis of Numerical Algorithms", Ph.D. thesis, Texas Univ. (Austin), 1990.

[30] J. Petrovic, I. Herrera, P. Lombardi, F. Schäfer and F. S. Cataliotti, New. J. Phys. 15, 043002 (2013).

[31] Nielsen, M. A. \& Chuang, I. L. Quantum Computation and Quantum Information. Cambridge University Press (2000). 


\author{
Nanometric Surface Probing through Ultra-Cold Atoms \\ Murtaza Ali Khan $^{(a, b)}$, Florian Schaefer ${ }^{(c)}$, Wolfram H. P. Pernice ${ }^{(b)}$ \\ Francesco S. Cataliotti ${ }^{(a)}$ \\ ${ }^{(a)}$ European Laboratory for Non-Linear Spectroscopy (LENS), via Nello Carrara 1, 50019 Sesto F.no (Firenze) - Italy \\ ${ }^{(b)}$ Karlsruhe School of Optics (KSOP), Schlossplatz 19, 76131 Karlsruhe - Germany \\ ${ }^{(c)}$ Department of Physics, Graduate School of Science, Kyoto University, 606-8502 Kyoto - Japan \\ Email: alikhan@lens.unifi.it
}

\begin{abstract}
We introduce a new experimental apparatus for cold atom based on an atom chip setup. It is going to feature for the first time the ability to interchange the atom chip frequently and rapidly. The setup will be paired with photonic structures on-chip for the detailed study of matter-light interactions. Here, we present the design of the new apparatus and present first ideas on how to use the unique combination of coldatom technology with interchangeable photonics components, both, for basic research and applications to modern information technologies.
\end{abstract}

Introduction - The advent of laser cooling techniques had a great impact on atomic physics and opened a door for the precise control of quantum states. Nowadays cold atoms can also be manipulated on the quantum level by using magnetic micro traps on a chip. These atom chips [1-3] are used to prepare cold atomic samples and Bose-Einstein condensates (BEC) in experimental setups that are more compact than traditional apparatus and allow for fast experimental cycles [4]. Further manipulation of the atoms, such as guided transport [1] and precise control of the quantum state [2], can be achieved by help of suitably micro fabricated wire patterns on the chip [3]. Additionally, integrated photonic structures fabricated in silicon thin films have gained importance in various applications [5-7] and, due to the small the band gap of silicon of $1.1 \mathrm{eV}$, these silicon-oninsulator systems have till now been used for, e.g. the creation of waveguides for wavelengths above $1100 \mathrm{~nm}$, benefiting also from low material absorption rates and good material properties [8]. For visible wavelengths, such as $780 \mathrm{~nm}$, photonic devices had to be realized in different materials that are compatible with silicon fabrication processes [9]. In this respect we introduce a new experimental setup that combines these two emerging technologies: atom chip based atomic physics with integrated photonic functionality in silicon technology to provide insight into the tailored control of light fields and photonic memories.

Experiment - In the experiment an atom chip will be mounted inside a glass cell allowing for high optical access to the photonic structures on the chip as well as for the creation and manipulation of a cold ${ }^{87} \mathrm{Rb}$ cloud. The glass cell has outer dimensions of $78.5 \mathrm{~mm} \times 80 \mathrm{~mm} \times 28 \mathrm{~mm}$, the glass thickness is $4 \mathrm{~mm}$. On one side of the cell a glass-to-metal transition tube is attached under $45 \mathrm{deg}$ (see Fig. 1). This tube of shortest possible length $(113.5 \mathrm{~mm})$ ends in a standard CF-40 flange, the whole assembly being ultra-high vacuum rated. The central components of the setup are shown in Fig. 1. Great care is taken to reduce the overall length of the setup between the glass cell and the UHV gate valve. This is to allow an easy replacement of the chip that is internally connected via a 
sturdy rod to a linear shift mechanism. We will employ the HLSM 38-300-SS linear shift stage with a maximum travel of $300 \mathrm{~mm}$. The UHV gate valve (model E-GV-1500M 302012 DN40CF) is open when the rod locates the chip inside the glass cell during normal experiment conditions. To replace the atom chip, the sustaining rod can be retracted from the cell and the gate valve closed. This realizes a load-lock system in which the central component of our experiment, the chip, can be exchanged without breaking the ultra-high vacuum on the glass cell side of the experiment. Upon re-installation of the chip the load-lock region will be brought back to high vacuum conditions via an integrated pumping access in the gate valve. The wires that establish the magnetic fields necessary for the magnetic trapping of $\mathrm{Rb}$ will be beneath the atom chip so that only the upper layer of the chip with the photonic structures needs to be replaced. The trapping setup is completed by external AntiHelmholtz coils for the initial magneto optical trap (M OT) stage and three pairs of Helmholtz coils to arbitrarily control homogeneous [10] magnetic fields (see Fig. 2). The analysis of the Anti-Helmholtz coils is shown in Fig. 3, field gradients of $8 \mathrm{G} / \mathrm{cm}$ are expected for currents of $15 \mathrm{~A}$. As conventional for atom chips we will employ a variation of the MOT technique known as mirror M OT [11].

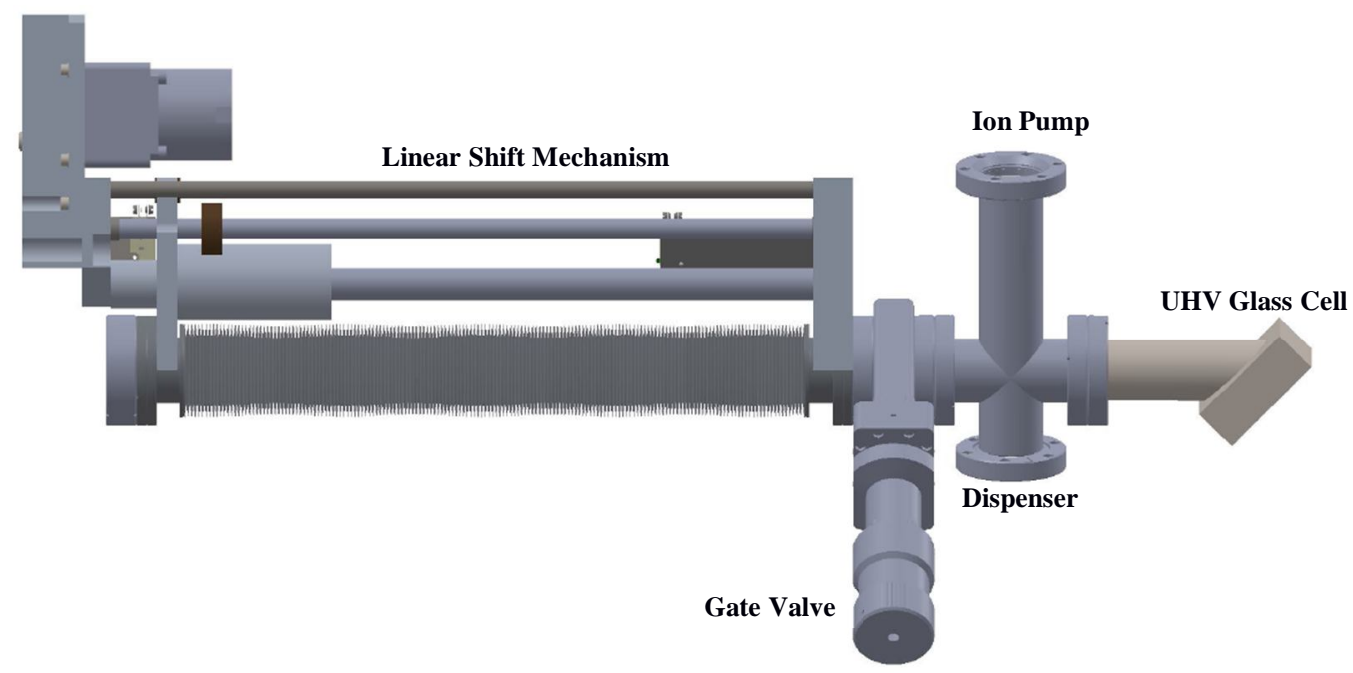

Fig. 1. Central vacuum components of the new setup. To the right the UHV glass cell is shown that will contain the atom chip. It is mounted under $45 \mathrm{deg}$ in respect to gravity to allow for time-of-flight imaging of the atomic sample. A custom $\mathrm{CF} 40$ cross provides access for ${ }^{87} \mathrm{Rb}$ dispensers and an ion pump. The gate valve is to realize the load-lock system when the chip is retracted by means of the linear shift mechanism. The distance between glass cell center and outer gate valve edge is $254 \mathrm{~mm}$. 


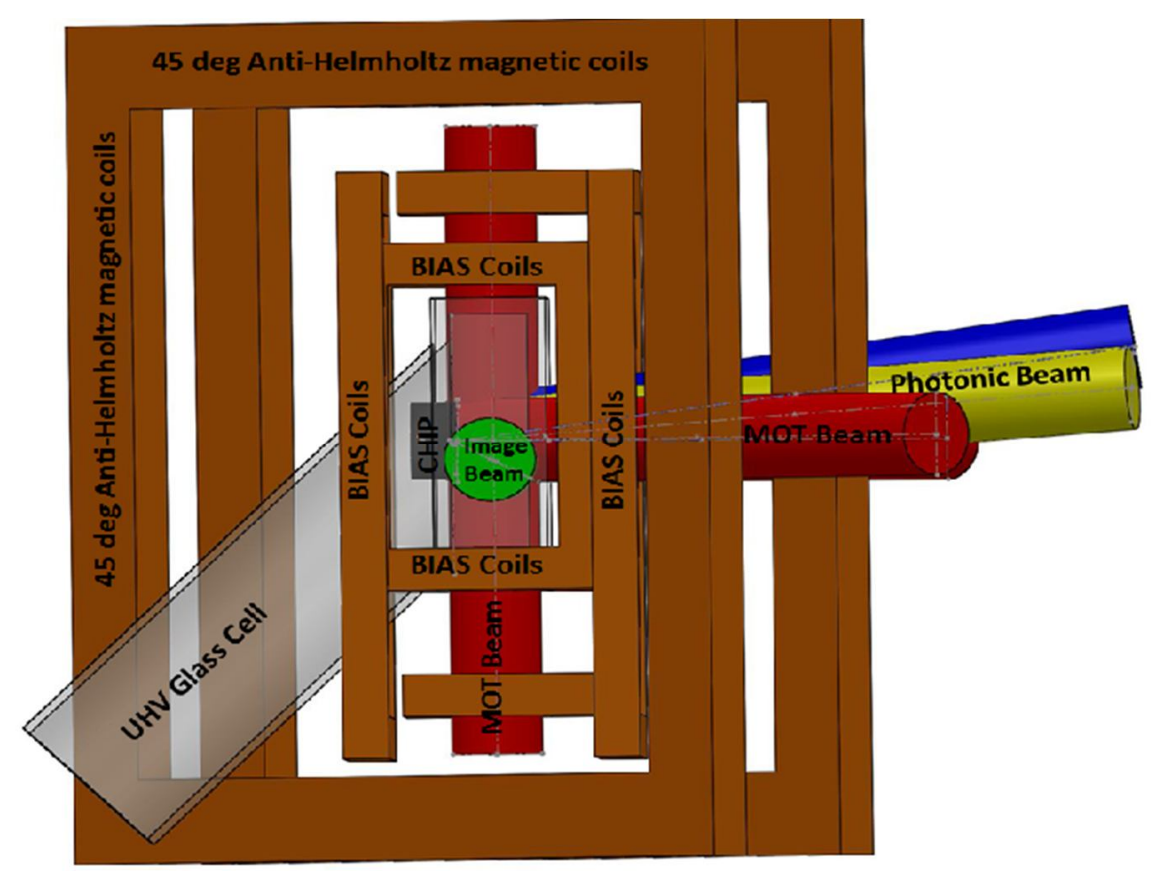

Fig. 2. Close up of the UHV glass cell part with magnetic coils and principal laser beams. Shown are schematically the MOT beams (red), the imaging direction (green) and the laser beams for excitation and probing (yellow and blue) of the photonic structures on-chip. The glass cell is surrounded by square coils for MOT (cf. also Fig. 3) and homogeneous bias fields.
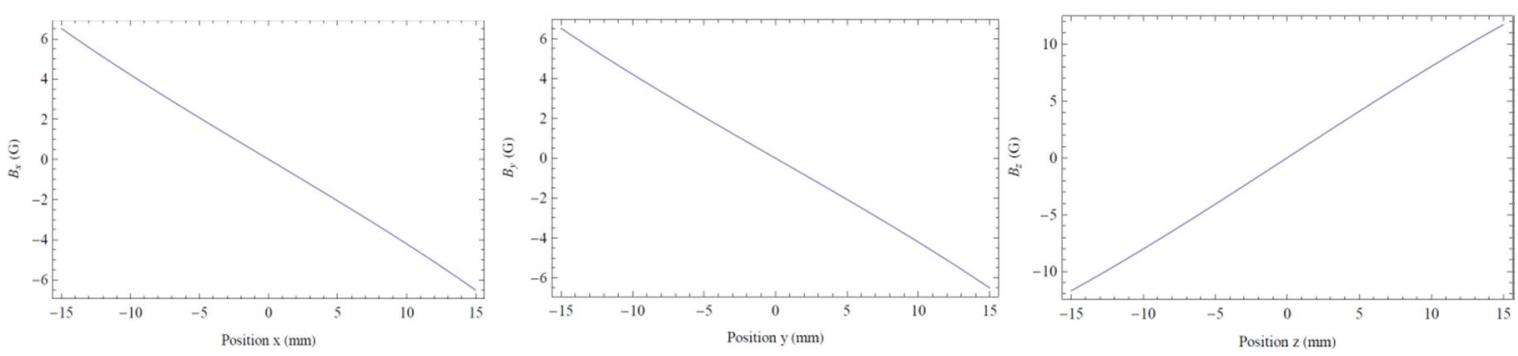

Fig. 3. Analysis of the Anti-Helmholtz coil pair. They will have square shape of $160 \mathrm{~mm}$ side length and $400 \mathrm{~mm}$ of mutual separation. With 80 turns of $2 \mathrm{~mm}$ diameter copper wire on each coil, magnetic field gradients of $8 \mathrm{G} / \mathrm{cm}$ can be realized at a current of $15 \mathrm{~A}$.

Nowadays, nanolithograpic tools allow to efficiently imprinting photonic structures onto a substrate. This standard industry technology allows realizing photonic structures in commercially available silicon nitride wafers with very high optical quality (Q) factors [30,000 for $780 \mathrm{~nm}$ wavelength]. Based on this technology we will design and fabricate an atom chip at KIT Germany. It consists of four basic layers of $\mathrm{Si}_{1} \mathrm{SiO}_{2}(2 \mu \mathrm{m}) \mathrm{SiN}(200 \mathrm{~nm})$ and copper $(\mathrm{Cu})$ as shown in Fig. 4. After structuring the SiN layer to implement the photonic functionality a gold layer is deposited on those areas of the chip without photonic functionality. This serves to increase the reflectivity of the surface needed for the realization of the mirror MOT. 


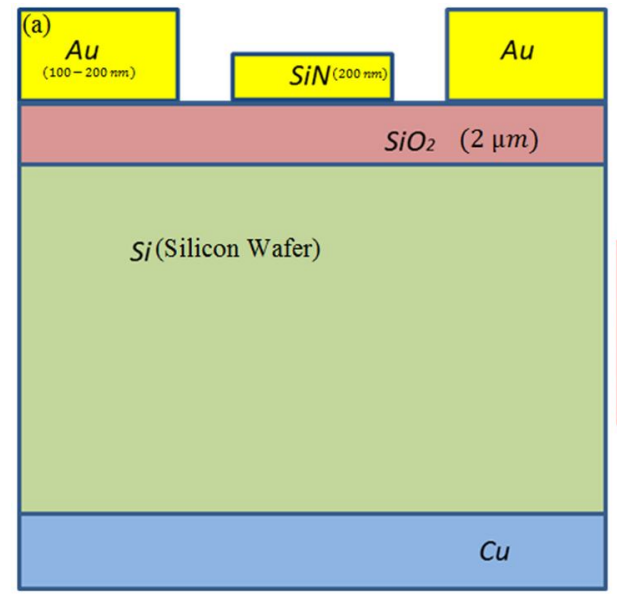

(b)

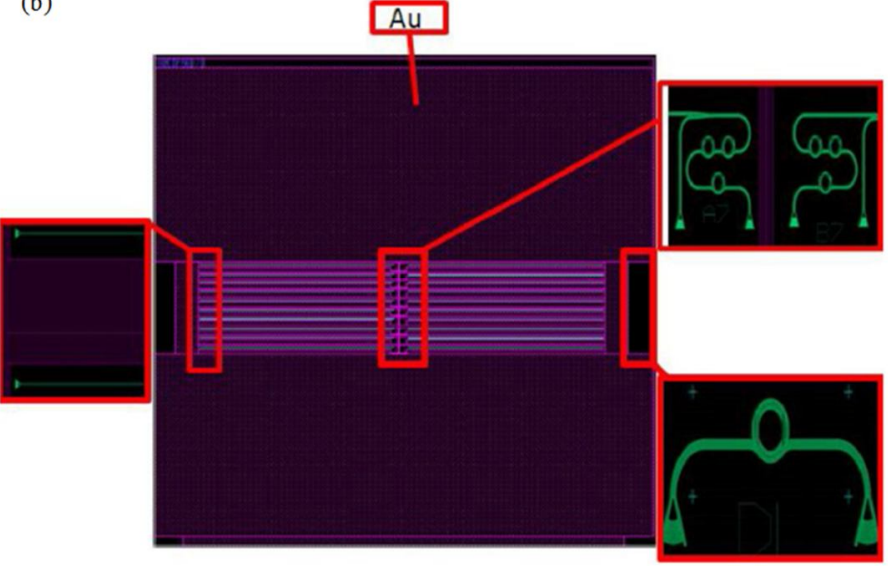

Fig. 4. Design of the atom chip: (a) Fabrication process as seen in a side view of the atom chip. The different layers are a copper $(\mathrm{Cu})$ base of the atom chip, a silicon wafer $(\mathrm{Si})$, a layer of silicon dioxide $\left(\mathrm{SiO}_{2}\right)$, the silicon nitride $(\mathrm{SiN})$ structure and a final gold ( $\mathrm{Au}$ ) coating for better surface reflectivity. (b) Top view of the design of the atom chip. Visible are the straight optical waveguides, the circular optical ring resonators and V-shaped structures to couple laser light into and out of the photonic structures.

In the first round of experiments we plan to implement optical ring resonators ( $40 \mu \mathrm{m}$ radius) connected via optical waveguides to light coupling structures. These grating couplers are used to couple the light into and out of the chip [12]. In a preliminary work three sets of grating couplers were already optimized for operation at $780 \mathrm{~nm}$ (the wavelength of the optical transitions used in experiments with ${ }^{87} \mathrm{Rb}$ ). By adjusting the period, Fig. $5(\mathrm{a}, \mathrm{b})$, of the grating it is possible to optimize the coupling efficiency of the couplers. In Fig. $5(d, e)$ we report the calculated performance achievable in a system of two couplers connected by a piece of waveguide, Fig. 5 (c).

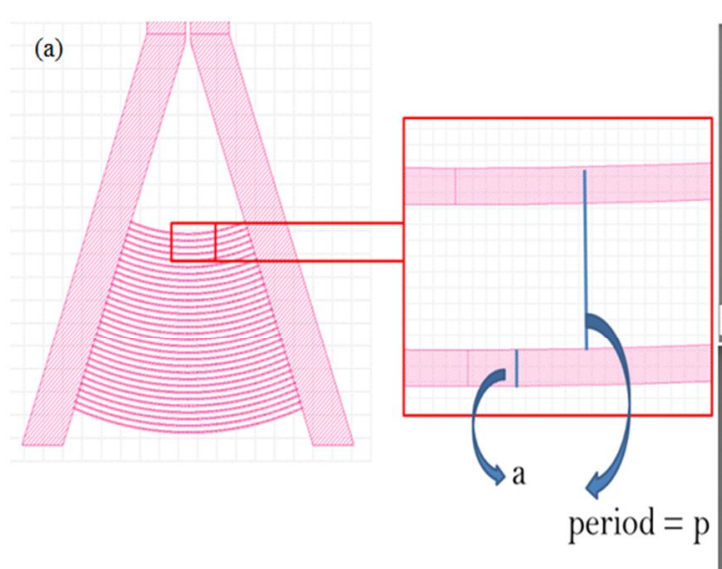

Fill factor $(\mathrm{ff})=\mathrm{a} / \mathrm{p} \cdot 100$

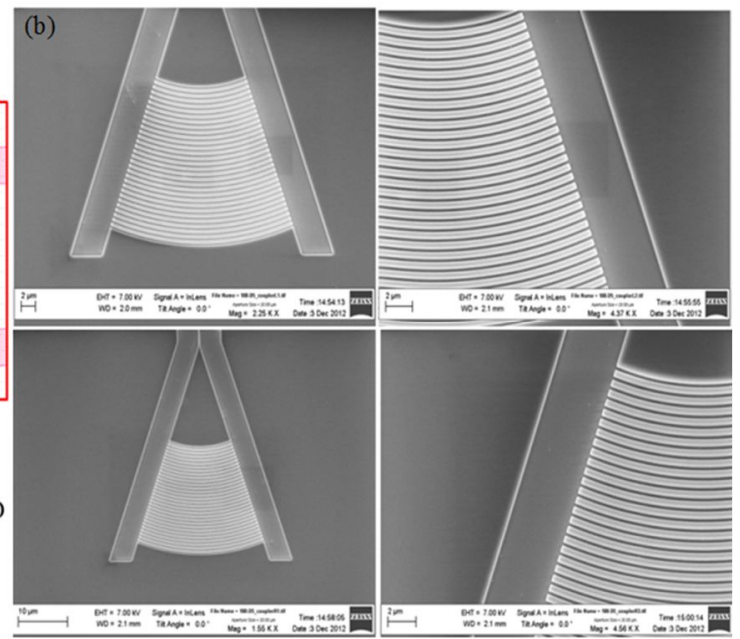



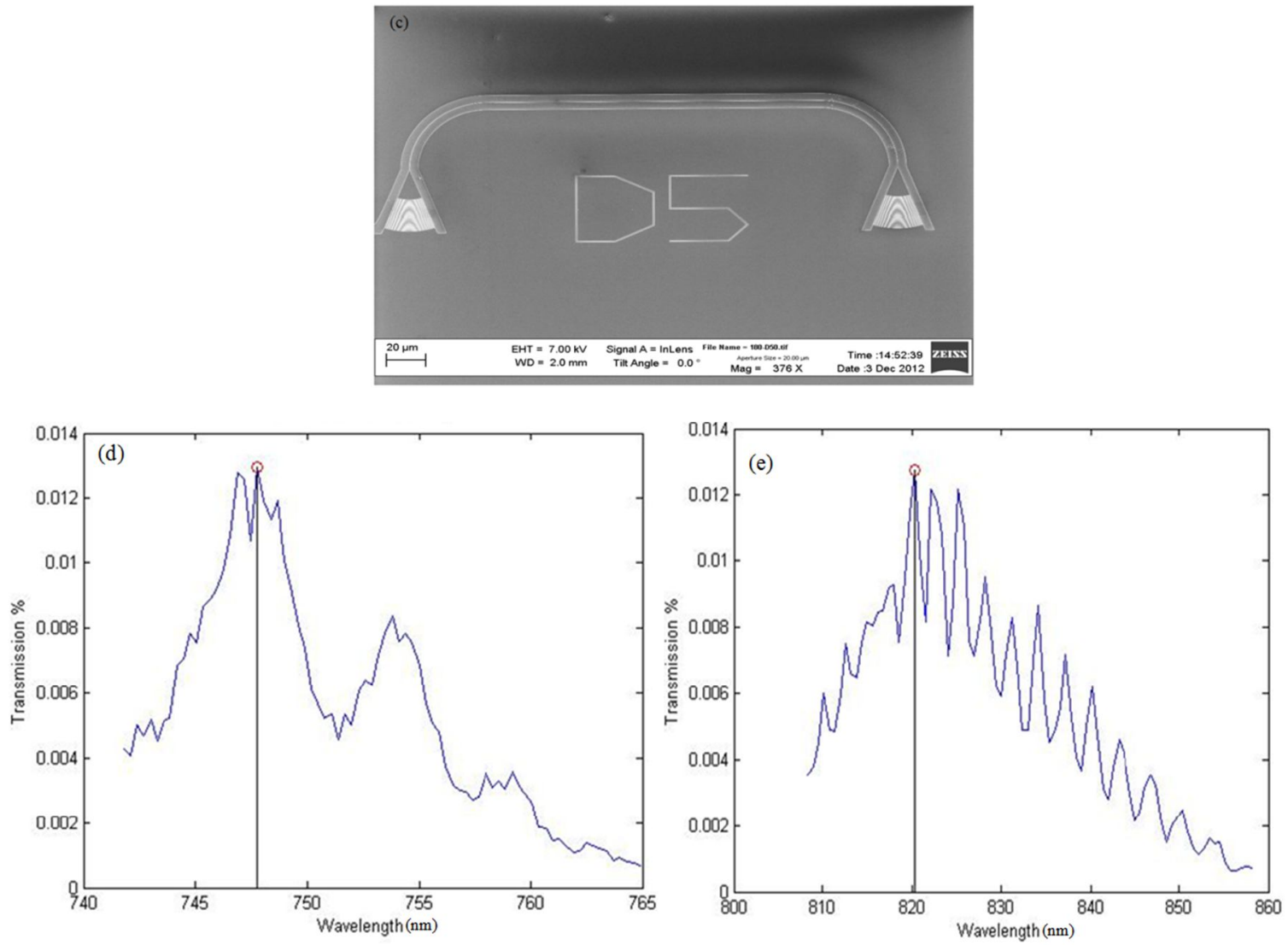

Fig. 5. Optimization of the light coupler design. (a) Schematics of the V-shaped light couplers, where the structure width $a$ and the repetition period $p$ are free parameters. (b) $2 \mu \mathrm{m}$ and $10 \mu \mathrm{m}$ SEM images of the couplers to verify the clean, roughness-free realization of the structures. (c) SEM image of two couplers connected by a waveguide. Visible light is later coupled to the left coupler, propagates along the waveguide and is coupled out by the right-side coupler. (d), (e) A high-sensitivity photo detector measures the transmission while the excitation wavelength is scanned.

While the light couplers are optimized to couple $780 \mathrm{~nm}$ laser light to the photonic structures the ring resonators feature exceptionally high $Q$ factors. Q factors in excess of 30000 were demonstrated [9]. For visible wavelengths (around $750 \mathrm{~nm}-810 \mathrm{~nm}$ ) a ring resonator is shown in Fig. 6. Optimized for a wavelength of about $780 \mathrm{~nm}$ the photonic structures will also be in resonance to optical transitions within ${ }^{87} \mathrm{Rb}$. Hence, an efficient coupling between the resonantly enhanced light fields in the optical ring resonators and the atomic levels is ensured and the mutual influence between the two key components of the new experiment can be studied in detail.

(a)

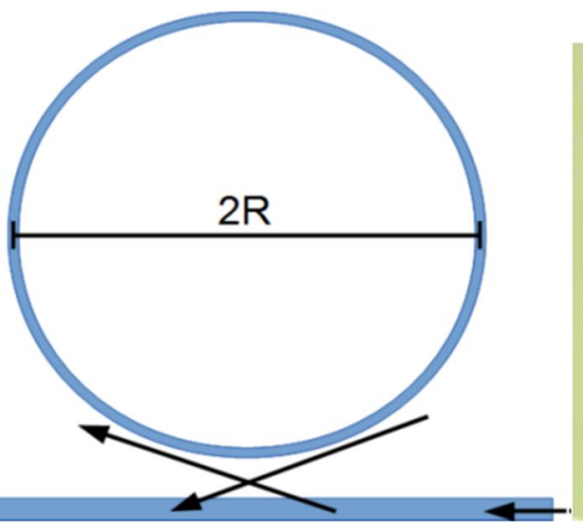

(b)

$80 \mu \mathrm{m}$ 
Fig. 5. Design of high Q factor optical ring resonators, (a) Schematic representation of the circular resonator coupled via evanescent fields to a close-by passing waveguide. (b) Image of a fabricated ring resonator for measuring the optical $\mathrm{Q}$ factor at near infrared and visible wavelengths. The distance between waveguide and optical ring resonator is $650 \mathrm{~nm}$.

Conclusions - The study of light-matter interaction is a basic tool in physics research. Furthermore, it is steadily gaining importance in applications. For future information technology applications the fast and minute control of quantum light fields is of basic interest and will be the basic building block of state-of-the art communication protocols. Our experiment is to combine available techniques in the field of atomic physics and photonics to precisely control, both, cold atoms and light fields. Our experimental efforts will provide the ideal platform for the study of matter-light interactions. First studies are to concern the influence of ${ }^{87} \mathrm{Rb}$ atoms on the $\mathrm{Q}$ factors of resonating structures. Applications in the field of optical switches and high-precision sensors are perceivable. Based on these results new chip designs with enhanced possibilities will be developed and, due to the modular load-lock system, rapidly put to study. Due to this unique property of our project, the ability to quickly exchange the atom chip, we are able to rather easily extend our studies to investigations of the behavior of plasmonic meta-materials and other interesting structures, such as carbon nanotubes, in close contact to atomic samples. Finally, while at present the experiment only aims to realizing a cold atoms cloud, the setup is versatile enough to enhance the chip functionality by embedding further electronic circuits that allows us to attain degenerate atomic samples, BoseEinstein condensates. In this realm even stronger mutual influences between light fields and matter waves are to be expected.

Acknowledgments - This work was supported by the Seventh Framework Programme for Research of the European Commission, under FET-Open grant MALICIA (265522), Murtaza Ali Khan is supported by Doctorate Euro-photonics Erasmus M undus program, and the experimental apparatus is based at the European Laboratory for Non-Linear Spectroscopy (LENS), University of Florence. The atom chip is fabricated at Karlsruhe School of Optics (KSOP), Karlsruhe Institute of Technology.

\section{References}

[1] J. Reichel, W. Hänsel, P. Hommelhoff, and T. W. Hänsch. Applications of integrated magnetic microtraps. Appl. Phys. B 72, 81-89 (2001).

[2] R. Folman, P. Krüger, J. Schmiedmayer, J. Denschlag, and C. Henkel. M icroscopic atom optics: From wires to an atom chip. Adv. At., M ol., Opt. Phys. 48, 263-356 (2002).

[3] József Fortágh and Claus Zimmermann. Magnetic microtraps for ultracold atoms. Rev. Mod. Phys. 79, 235-289 (2007).

[4] Recently atom chip based BECs became available from ColdQuanta Inc. Boulder (CO).

[5] R. Kirchain, and L. Kimerling. A roadmap for nanophotonics. Nat. Photon. 1, 303-305 (2007).

[6] B. Jalali, and S. Fathpour. Silicon photonics. J. Lightwave Tech. 24, 4600-4615 (2007).

[7] R. Soref. The past, present and future of silicon photonics. IEEE J. Sel. Top. Quantum Electron. 12, 1687-1687 (2006).

[8] V. P. Popov, A. I. Antonova, A. A. Frantsuzov, L. N. Safronov, G. N. Feofanov, O. V. Naumova, and D. V. Kilanov. Properties of Silicon-on-Insulator Structures and Devices. Semiconductors, Vol. 35, No. 9, 2001, pp. 1030-1037.

[9] W. H. P. Pernice, C. Xiong and H. X. Tang. High Q micro-ring resonators fabricated from poly crystalline aluminum nitride films for near infrared and visible photonics. Opt. Express 20, 12261-12269 (2012). 
[10] E. L. Raab, M. Prentiss, Alex Cable, Steven Chu, and D. E. Pritchard. Trapping of Neutral Sodium Atoms with Radiation Pressure. Phys. Rev. Lett. 59, 2631-2634 (1987).

[11] J. Reichel, W. Hänsel, and T. W. Hänsch. Atomic Micromanipulation with Magnetic Surface Traps. Phys. Rev. Lett. 83, 3398 (1999).

[12] D. Taillaert, P. Bienstman, and R. Baets. Compact efficient broadband grating coupler for siliconon-insulator waveguides. Opt. Lett. 29, 2749-2751 (2004). 


\section{A. Appendix: Vacuum Design}

Appendix A represents three Figures that are showing the drawings of three components of our experiment; Figure 59 shows the drawing of UHV glass cell. This cell is represented in Figure 17. Glass cell is the back bone of the cold atom experiment. In Figure 59 we constitute every parameter of the cell for in depth understanding regarding cell. Second drawing in Appendix A shows the four cross pipe that we already represented in Figure 20. All the values of four cross pipe is defined in Figure 60, Last drawing in Appendix A giving the description of metal rod, our Atom chip will be attached with this metal rod. Metal rod with CF-40 flange is shown in Figure 61. 


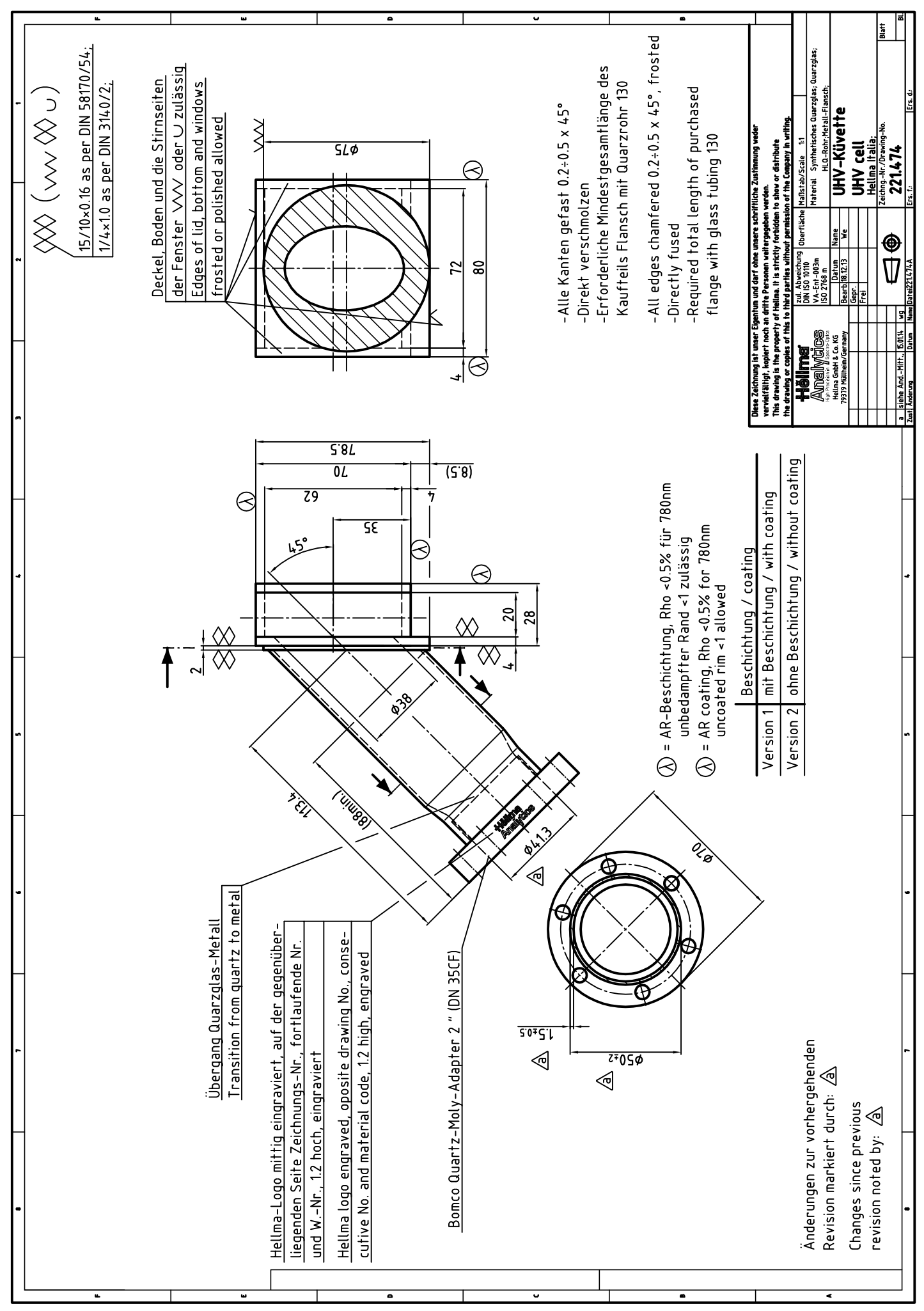

Figure 59: UHV Glass Cell with all parameters. Representation of Cell shown in Figure 17 


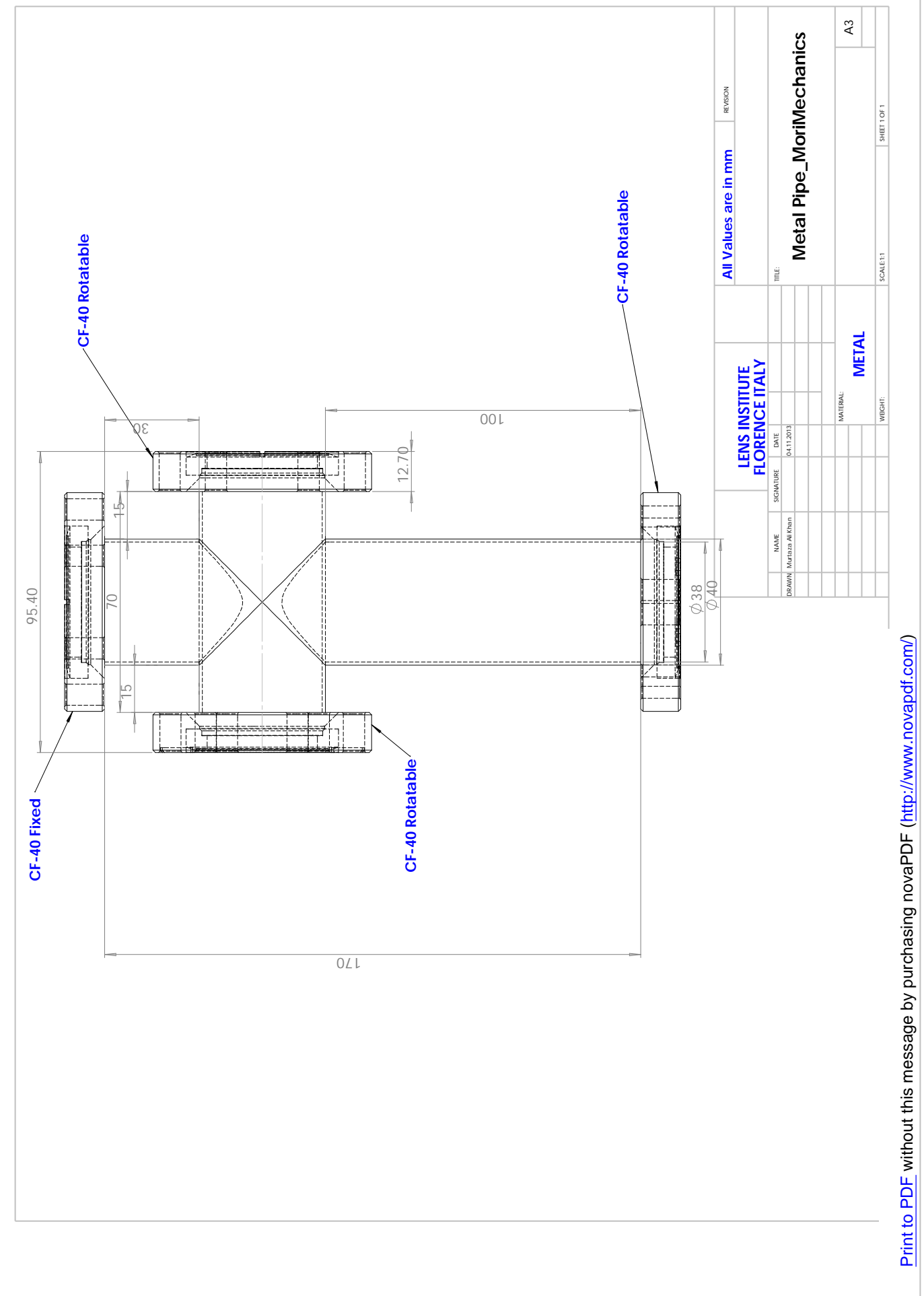

Figure 60: Drawing of four cross metal road, each opening of the pipe attached with CF-40 flange. Pipe attached with glass cell is shown in Figure 18. 


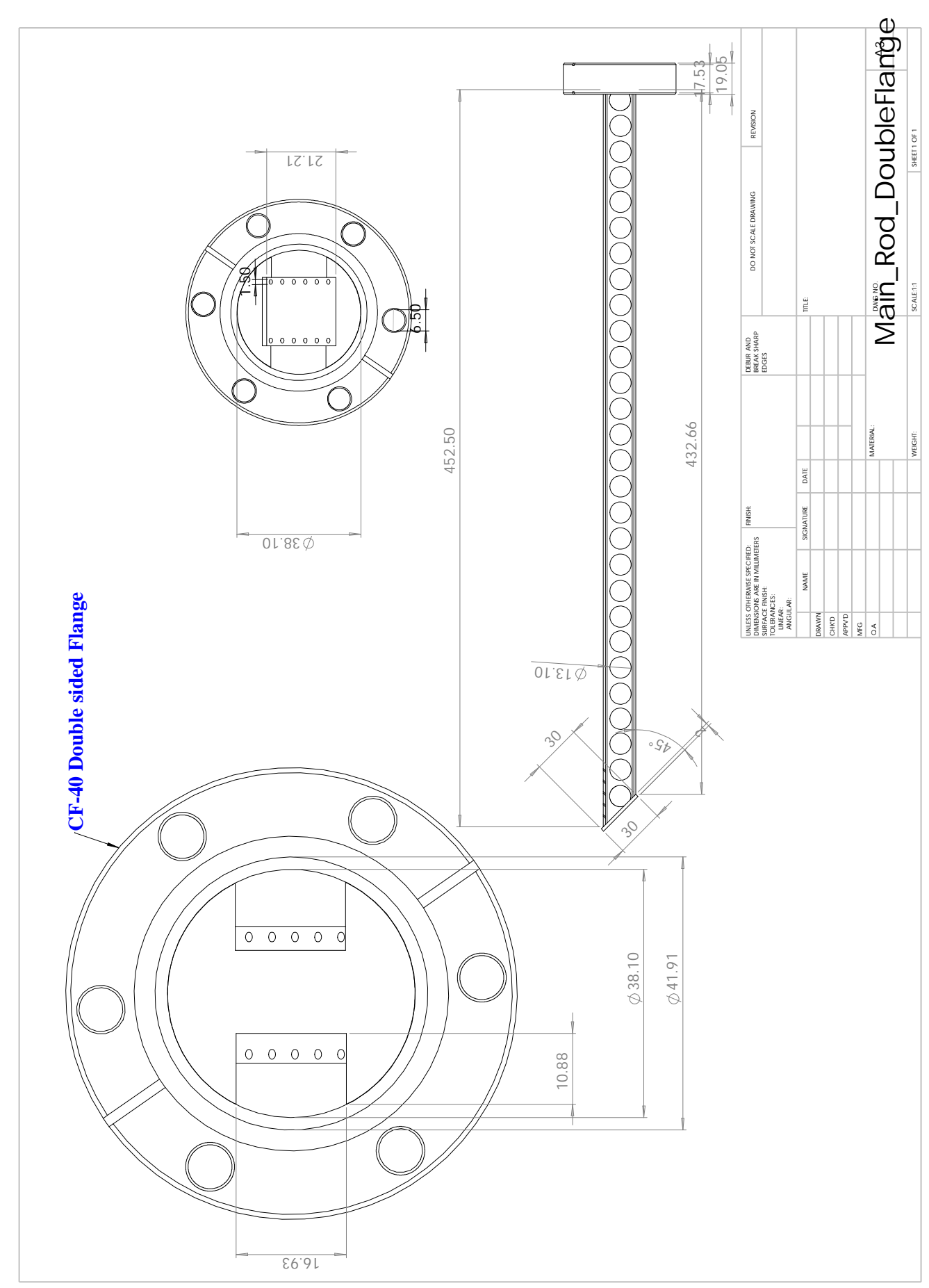

Figure 61: Metal rod drawing that showed in Figure 20 


\section{B. Appendix: Experiment Design}

Appendix B maneuvering between the clamps and their bars. These clamps are going to used to support the set up. We used three different types of clamps as per requirement of our set up. These clamps and bar are manufactured by Mori mechanica italia. Each clamp is designed to give a support of CF-40 flanges. The bar will be come on the clamp. Figure 63, 64, 65, 66, 67, 68 and 69 showing the clamps and bars respectively. 


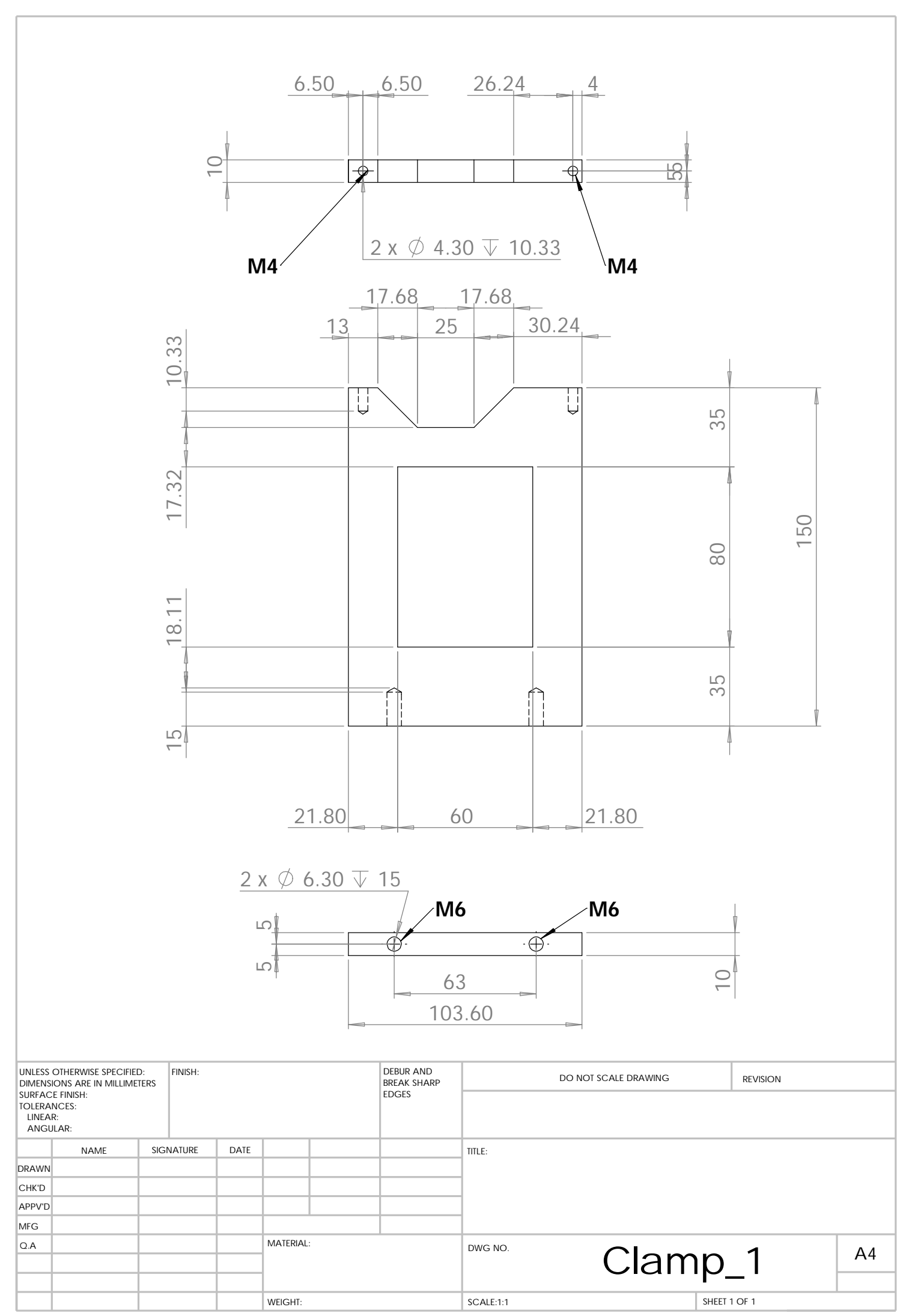

Figure 62: Clamp 1, drawing that will showed in Figure 30. Clamp 1 will give the support to the set up. 


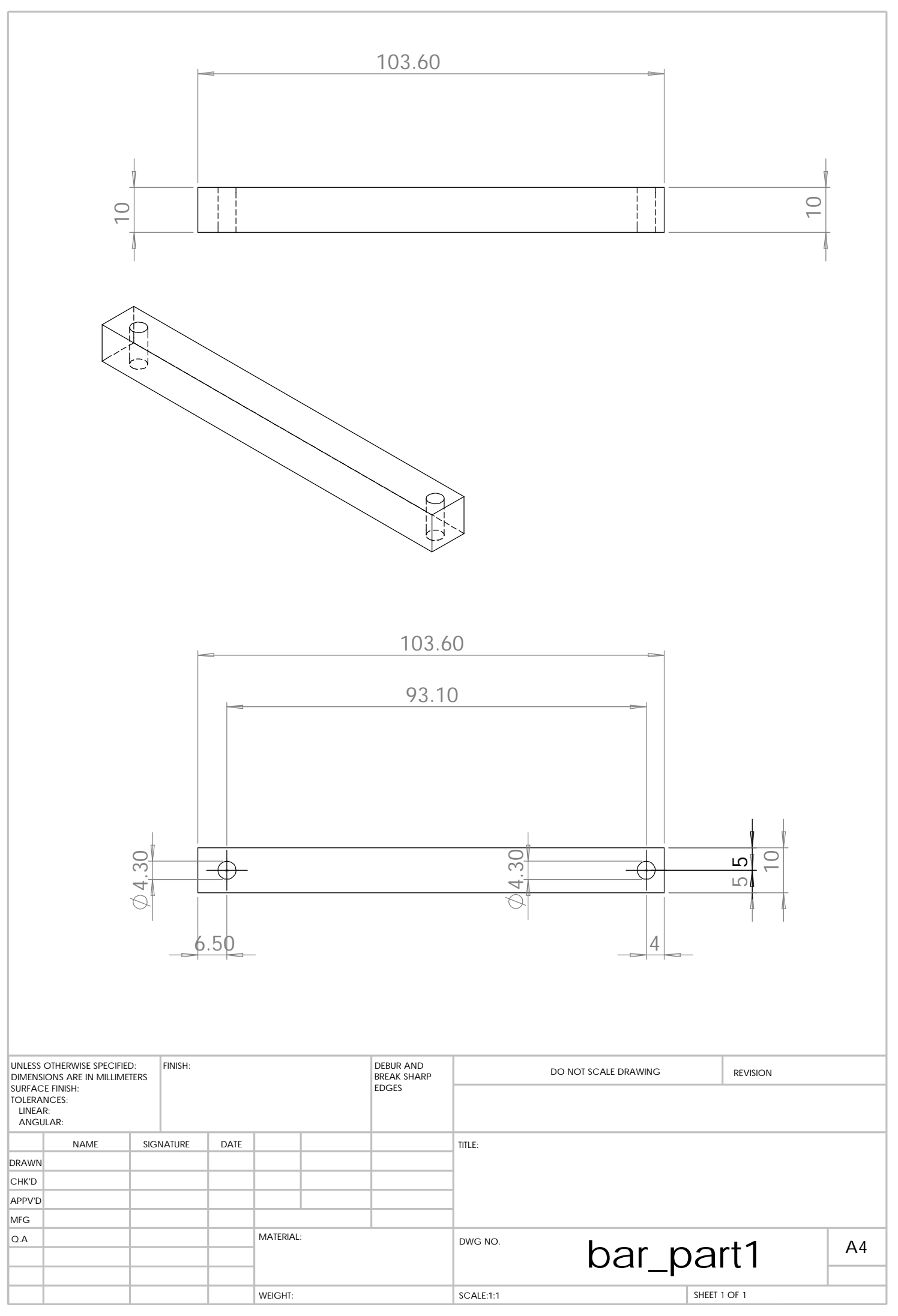

Figure 63: Bar of the clamp that will attached to the clamp 1. 

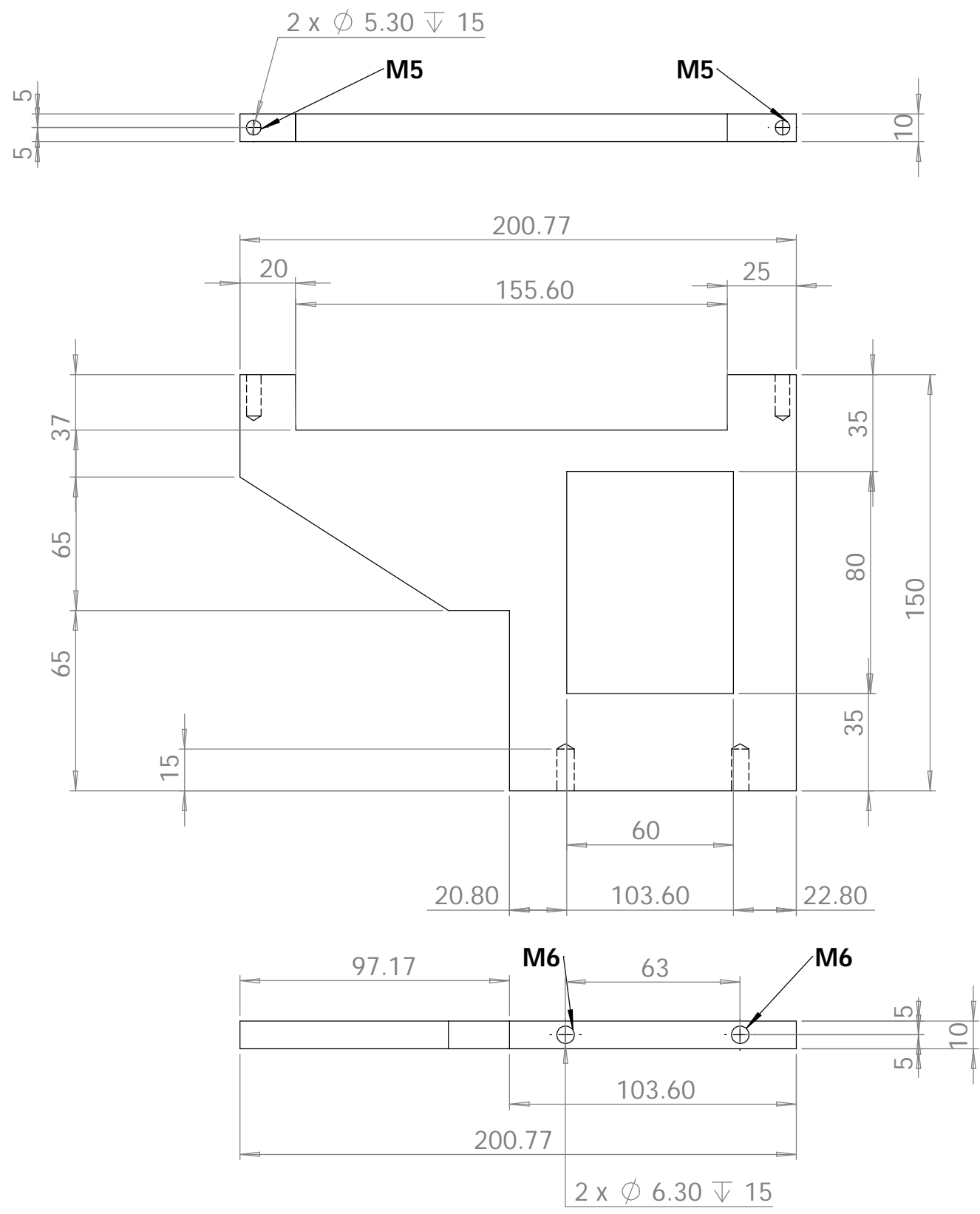

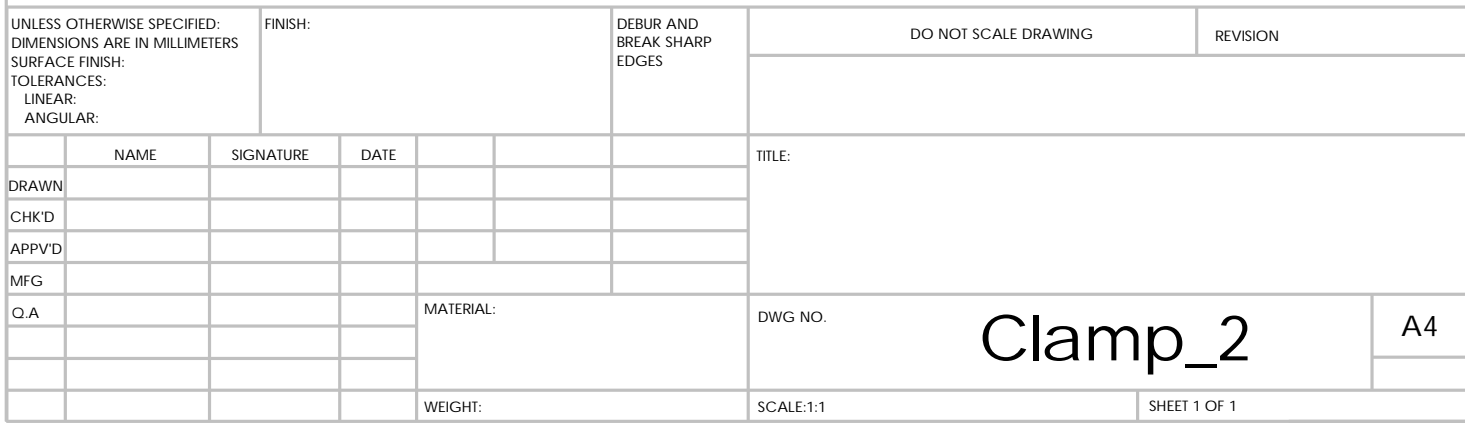

Figure 64: Clamp 2 also showed in Figure 30 


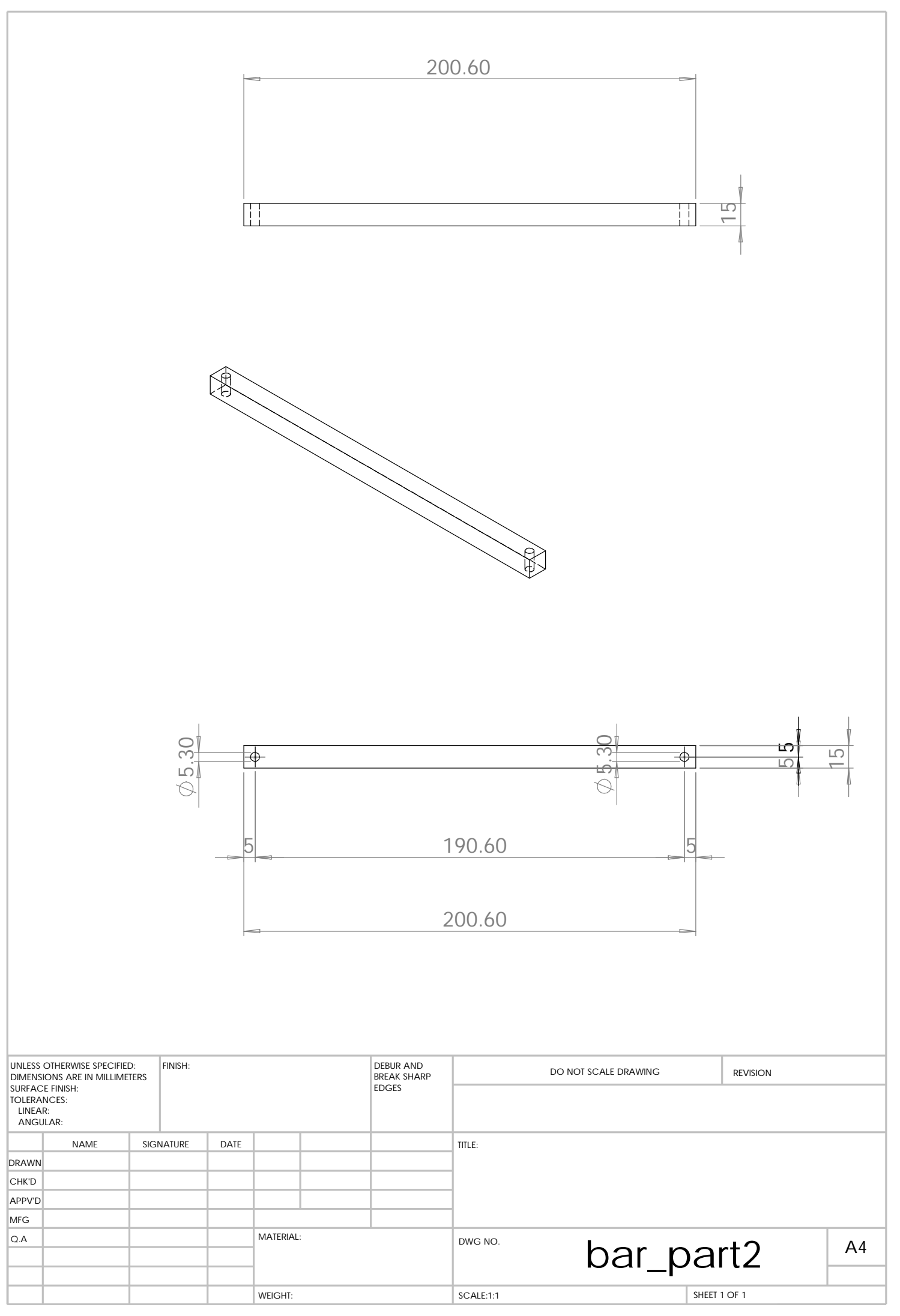

Figure 65: Bar of the clamp that will attached to the clamp 2. 


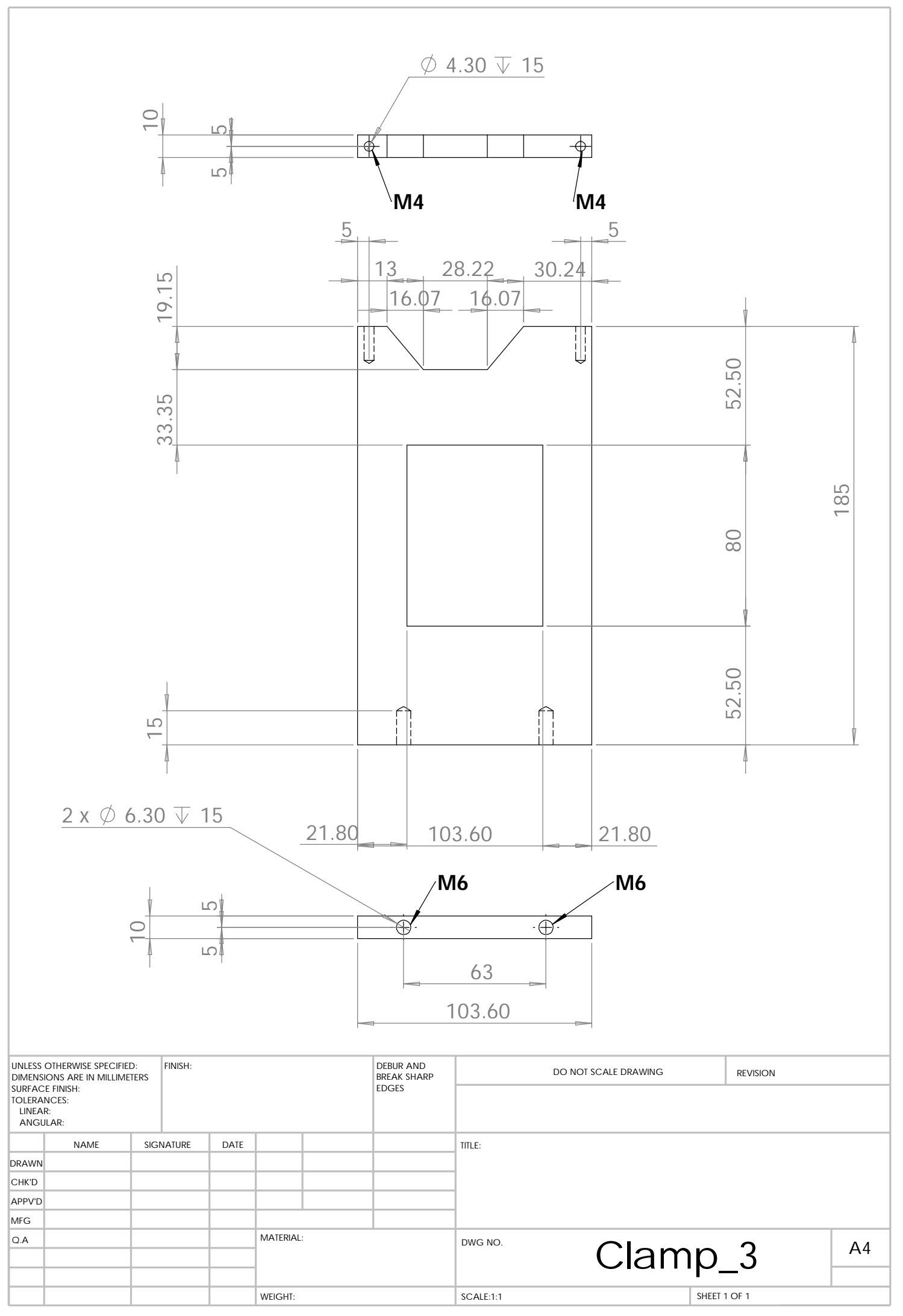

Figure 66: Clamp 3 for supporting the set up. 


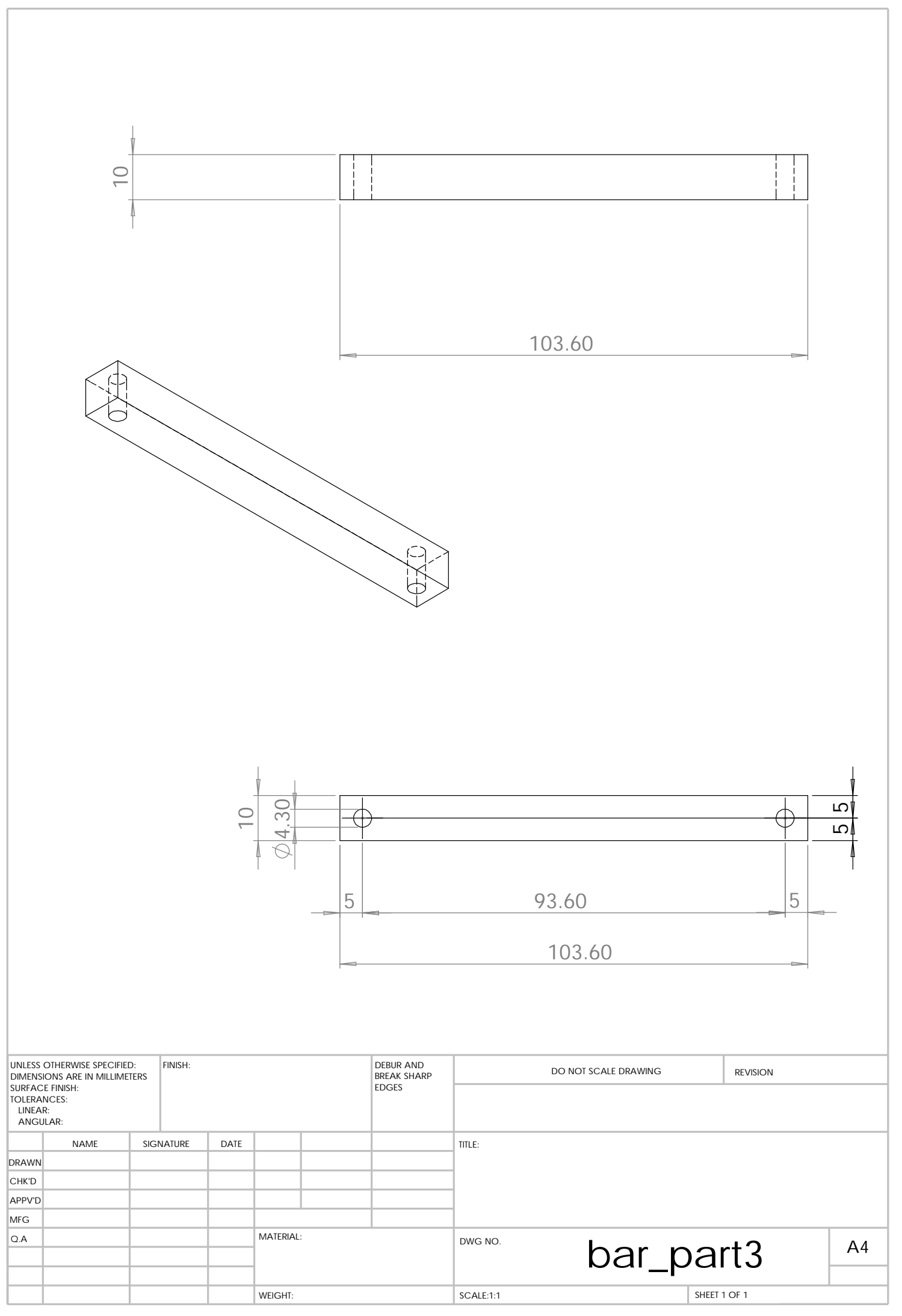

Figure 67: Bar of the clamp that will attached to the clamp 3. 


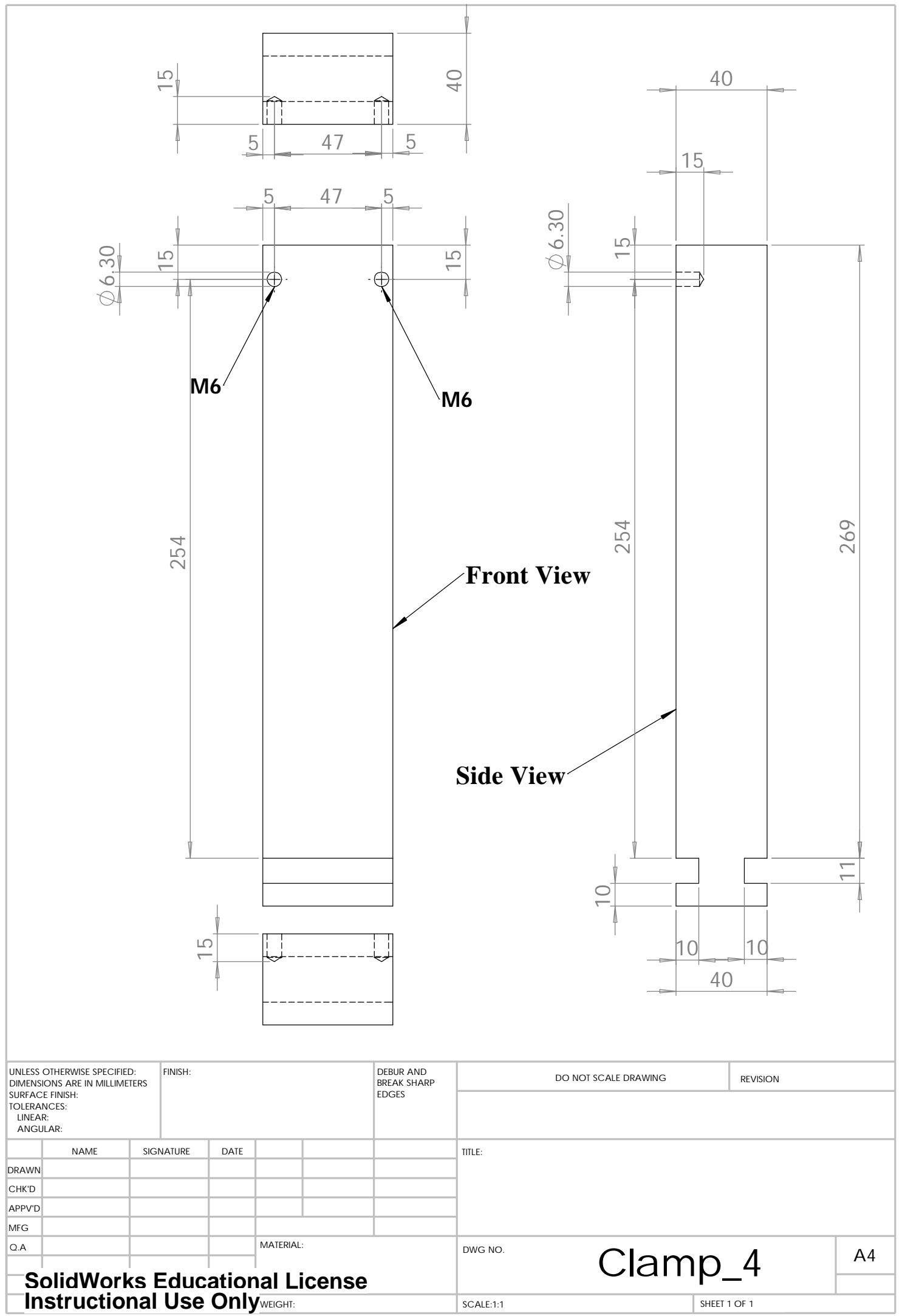

Figure 68: Metal bar supporting the glass cell. 


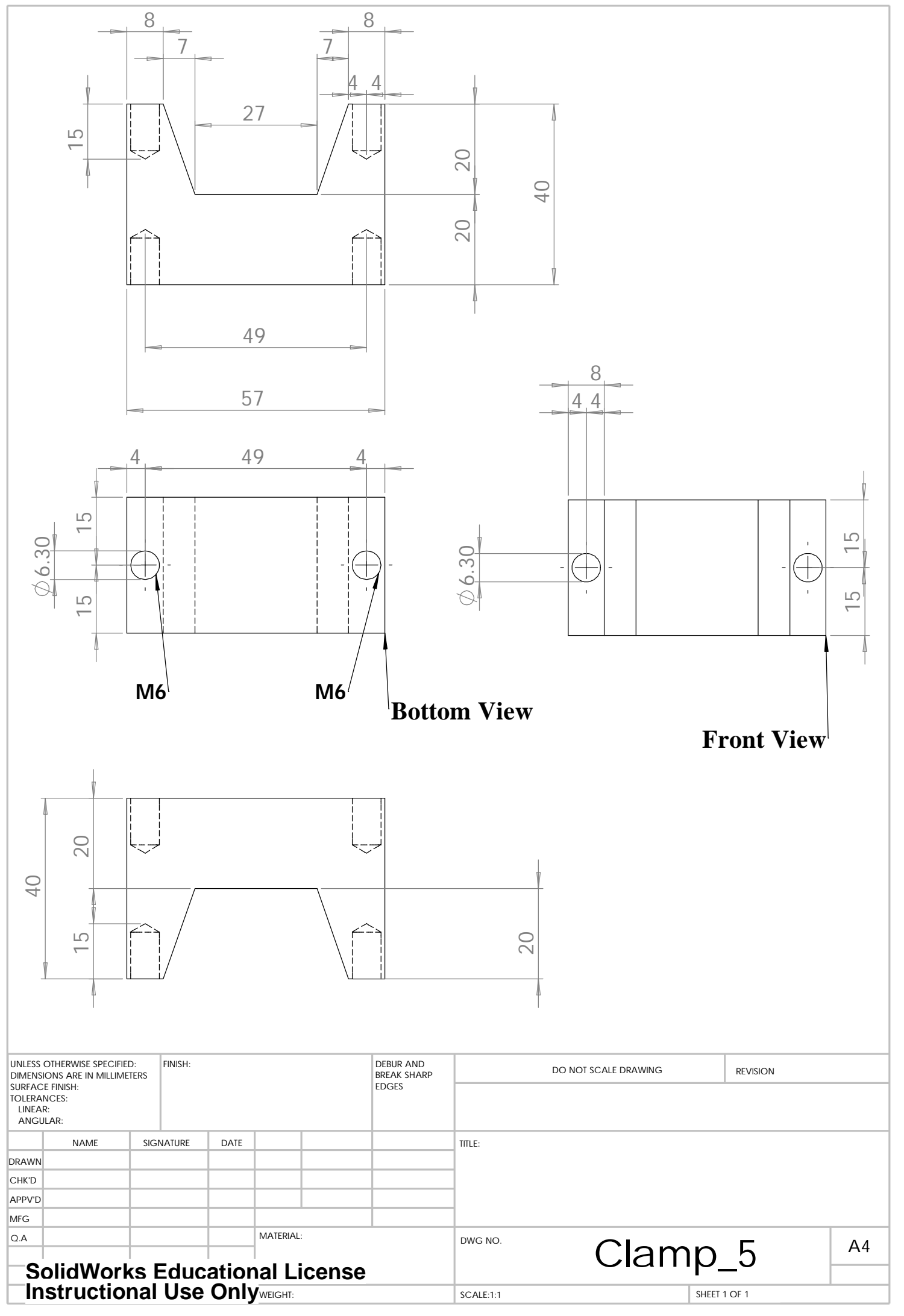

Figure 69: Bar that will support the metal rod. 